\title{
DIGITALCOMMONS
}

@WAYNESTATE-

Wayne State University

Wayne State University Dissertations

January 2019

\section{The Use Of Cultural Algorithms To Learn The Impact Of Climate On Local Fishing Behavior In Cerro Azul, Peru}

Khalid Kattan

Wayne State University, khalidkattan@gmail.com

Follow this and additional works at: https://digitalcommons.wayne.edu/oa_dissertations

Part of the Artificial Intelligence and Robotics Commons, and the History of Art, Architecture, and Archaeology Commons

\section{Recommended Citation}

Kattan, Khalid, "The Use Of Cultural Algorithms To Learn The Impact Of Climate On Local Fishing Behavior In Cerro Azul, Peru" (2019). Wayne State University Dissertations. 2170.

https://digitalcommons.wayne.edu/oa_dissertations/2170

This Open Access Dissertation is brought to you for free and open access by DigitalCommons@WayneState. It has been accepted for inclusion in Wayne State University Dissertations by an authorized administrator of DigitalCommons@WayneState. 


\section{THE USE OF CULTURAL ALGORITHMS TO LEARN THE IMPACT OF}

CLIMATE ON LOCAL FISHING BEHAVIOR IN CERRO AZUL, PERU

by

\section{KHALID KATTAN \\ DISSERTATION}

Submitted to the Graduate School

of Wayne State University,

Detroit, Michigan

in partial fulfillment for the requirements

for the degree of

DOCTOR OF PHILOSOPHY

2019

MAJOR: COMPUTER SCIENCE

Approved By:

Advisor

Date 
(C) COPYRIGHT BY

KHALID KATTAN

2019

All Rights Reserved 


\section{DEDICATION}

To my entire family, my Wife, Dhuha, my Daughters Malak, Deena, and Ayah, and my Son, Mohammed, I dedicate this work. Also, I dedicate this work to my Father, Dr. Ibrahim, my Mother, Siham, and to all my Sisters, Dr. Halide, Alya, Huda, Mona. 


\section{ACKNOWLEDGMENTS}

First and foremost, I am grateful to Almighty God for all things for which I and my family is blessed with through His grace. I am thankful for this opportunity, including the challenge of balancing family and research. I believe this had made me more compassionate and allowed me to grow.

My special thanks and sincere gratitude go to my advisor, Dr. Robert G. Reynolds for all his sacrifices, relentless dedication, patience and guidance along my long road to finalizing my thesis. Dr. Reynolds went above and beyond what is required and what is expected of a PhD advisor. Dr. Reynolds has the ability to bring the strengths of each of his individual students.

In addition, I would like to thank all additional members of my graduate committee: Dr. Schwiebert, Dr. Grosu, Dr. Marcus, and Dr. Flannery, for their advice, suggestions, and commitment. Their broad and invaluable expertise each helped to improve my Thesis. Also, I would like to thank Dr. Thaer Jayyousi, who has supported me for many years with his help and reassurance.

Also, I would like to especially thank Dr. Joyce Marcus and Dr. Kent Flannery from the University of Michigan, for providing their expert knowledge and invaluable insight on the Cerro Azul project. I am really thankful to Dr. Marcus for sharing their extensive data collection which expanded several years.

I am also grateful to all my professors with whom I have had courses; they have enriched my knowledge and skills during my study. I would like to thank current and previous members of the Computer Science Department and the Artificial Intelligence Laboratory for their efforts, assistance and research, which include work of, Dr. Mostafa Ali, 
Dr. Dapeng Lie, Dr. Thaer Jayyousi, Chris Best, and Dr. Bin Peng. My work is based on their previous achievements and research. I would also like to thank fellow colleagues Thomas Palazzalo and Dustin Stanley, both of whom provided assistance. Stanley developed a novel CA with PSO system, "CAPSO", and allowed me to benchmark my results in his system. Additionally, Stanley shared key ideas some of which have been included in this thesis.

Last but not least, my family. I am very thankful to my wife, Dhuha, for always standing by my side, for making many personal sacrifices, and for always supporting me during this endeavor. I thank my father, Dr. Ibrahim Kattan, for his continuous support and motivation. Also I am grateful for my mother, Siham Kattan, for her encouragement, and my sisters, Dr. Halide, Alya, Huda, and Mona for all their support. 


\section{TABLE OF CONTENTS}

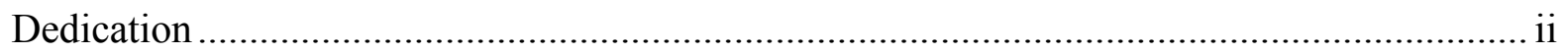

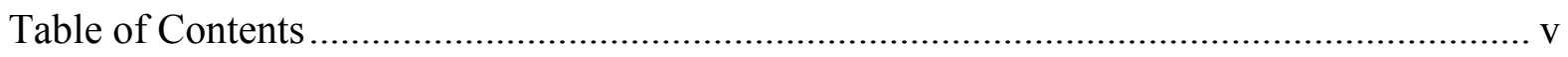

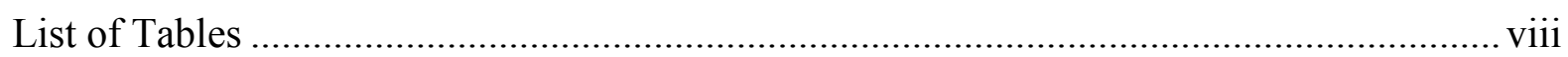

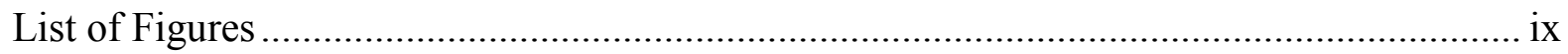

Chapter 1: Introduction and Motivation .......................................................................... 1

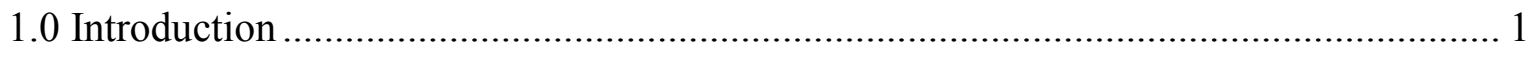

1.1 The Cerro Azul Ecosystem ……………………….............................................. 3

1.2 ENSO: An Example of Short Term Climate Change................................................... 9

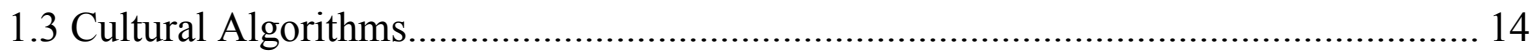

1.4 Multi-Objective Problem Statement...................................................................... 18

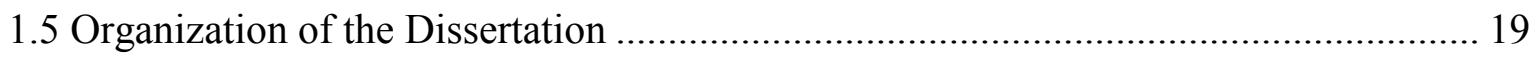

Chapter 2: An Overview of the Cerro Azul Fishing Data Set ................................................ 21

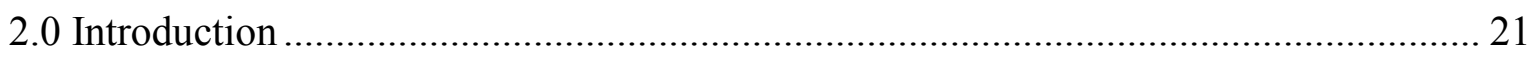

2.1 An Overview of the database content as A Complex System........................................ 22

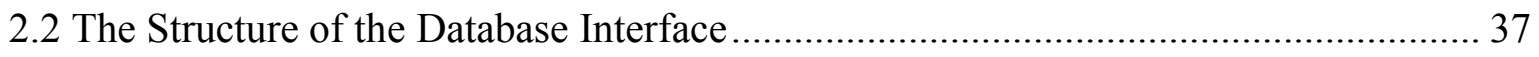

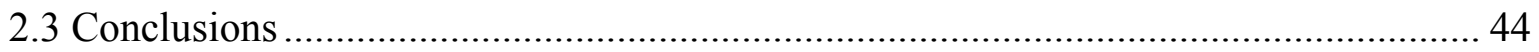

Chapter 3: Data Mining At the Meso and Micro Levels .......................................................... 46

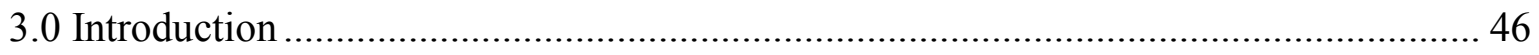

3.1 The-Level: Monthly and Daily Changes in Quality.................................................. 47

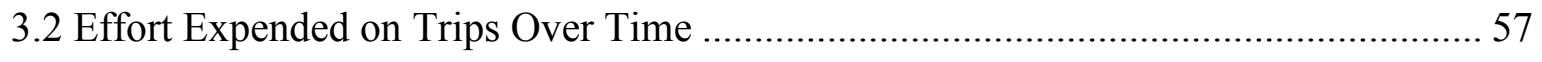

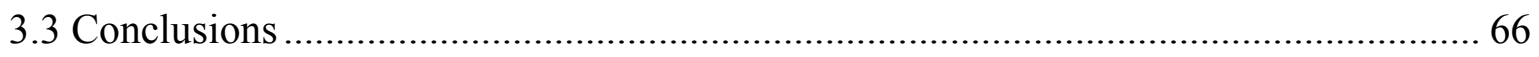

Chapter 4: Cultural Algorithms and Multi-Objective Optimization....................................... 68 


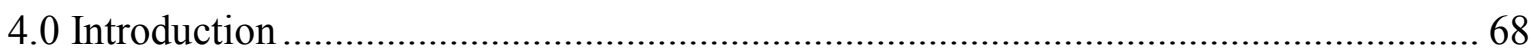

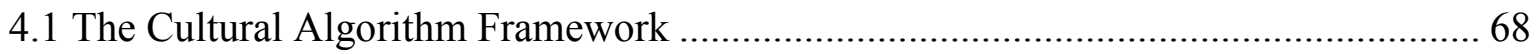

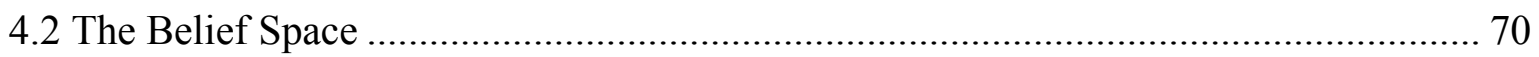

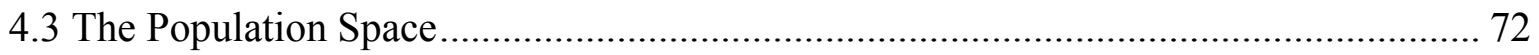

4.4 The Communication Protocol ……………………............................................... 74

4.5 Historical Development of Cultural Algorithms ......................................................... 75

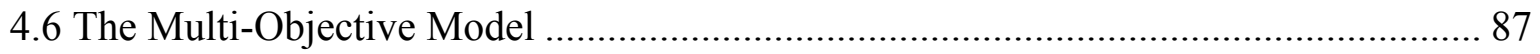

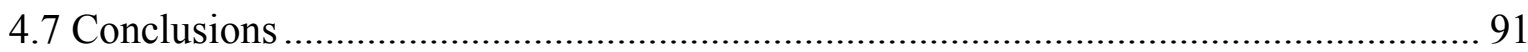

Chapter 5: Artisanal Fishing Model..................................................................................... 93

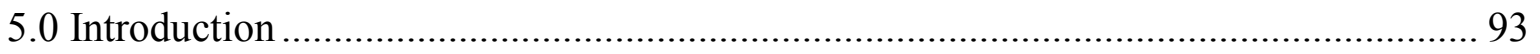

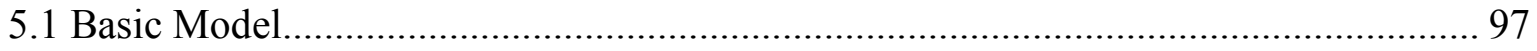

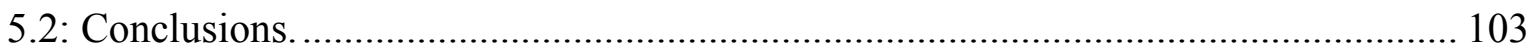

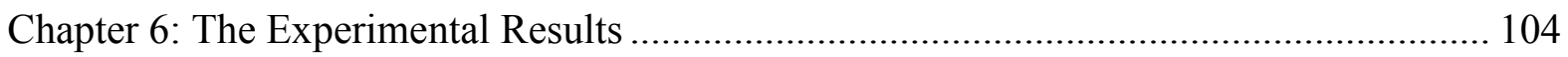

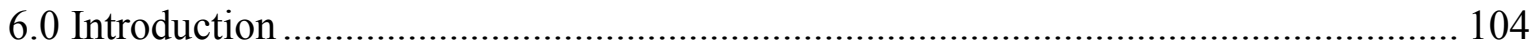

6.1 Comparison of Pareto Frontiers for Phase I........................................................... 105

6.2 Comparison of Pareto Frontier for Phase II. ............................................................... 116

6.3 Comparison of Pareto Frontiers for Phase III, Back to Normal.................................. 127

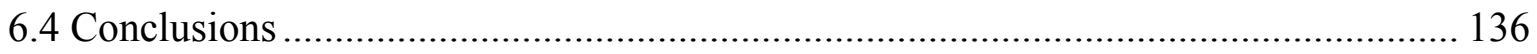

Chapter 7: Statistical Analysis of the Model Predictions for All Phases................................ 138

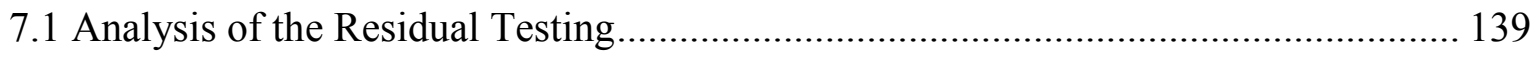

7.2 Comparison of Curve Fitting and Residual Phase I................................................... 139

7.3 Comparison of HYper-linear and linear model predictions IN PHASE II ................. 145

7.4 Comparison of hyperlinear curves and Residuals in Phase III................................... 150 
7.5 The Significant F-Test Analysis..................................................................... 156

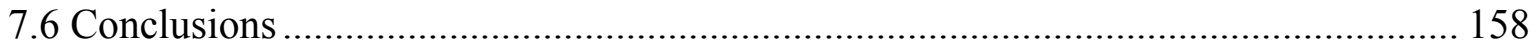

Chapter 8: Conclusion and Future Work .................................................................. 160

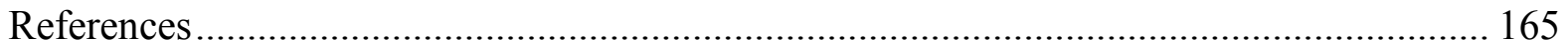

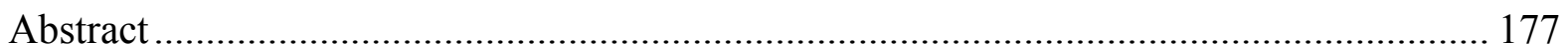

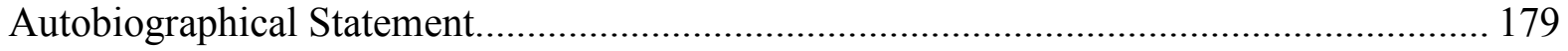




\section{LIST OF TABLES}

Table 2.1: Data Dictionary of the Peru Database ............................................................ 26

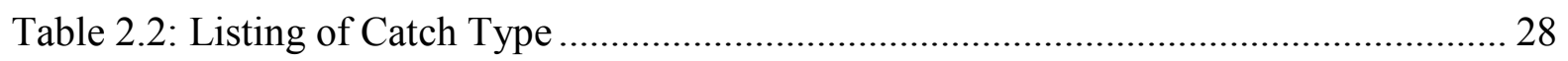

Table 2.3: Temporal Categories for Phases I. II and III .............................................. 29

Table 2.4: The Catch list, type, and desirability for all three Phases................................ 30

Table 2.5: The Site Distances from Cerro Azul.............................................................. 31

Table 2.6: The Trip Count for each Catch Type by Phase............................................... 32

Table 2.7: Trip Count based for Catch Type, Sorted by Trip Count by largest to smallest ... 34

Table 2.8: The Trip Count and corresponding percentage for each Site by Phase............... 36

Table 3.1: Species catch during each phase for desirability ........................................... 48

Table 3.2: The statistics for Catch Count for the entire period........................................ 51

Table 3.3: Total Catch Count by month for High Desirability....................................... 54

Table 3.4: Total Catch count by month for Okay Desirability. ....................................... 55

Table 3.5: Total Catch count by month for Fall Back Desirability ................................... 56

Table 3.6: The distance from Cerro Azul to all sites ........................................................ 58

Table 3.7: Trip Count over Entire Period Based upon Return Date. .................................... 60

Table 3.8: Catches by Phase for Entire Period using Return Date .................................... 62

Table 7.1: The Critical value for F-Test at different significant level of $\alpha$...................... 156

Table 7.2: Statistical value for $\left(\mathrm{F}_{\text {stat }}\right)$ for Phase I, II and Phase III. .................................. 156 


\section{LIST OF FIGURES}

Figure 1.1: The site location of Cerro Azul location, Yellow Star inside Yellow Circle........ 3

Figure 1.2: The Topographical Map of Peru. Cerro Azul is in the yellow circle................... 4

Figure 1.3: Relative location of modern day and prehistoric Cerro Azul [1] ...................... 6

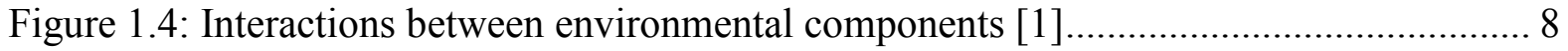

Figure 1.5: Normal conditions for circulation of the heat. [9] ........................................ 9

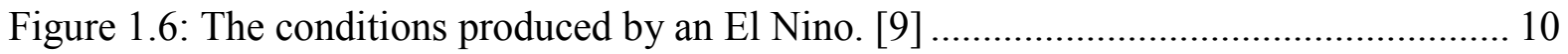

Figure 1.7: A heat map comparing the powerful El-Nino of 1997 to 2015 ........................ 12

Figure 1.8: Monthly avg. temperatures at Callu, Peru from 1981 to 1986 [1] .................... 13

Figure 1.9: La Nina and its cooling affect in blue. ...................................................... 14

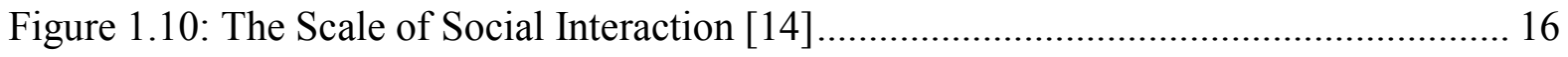

Figure 2.1: The different levels of data analysis: Macro, Meso and Micro......................... 25

Figure 2.2: Catch Count by week for Mismis............................................................... 35

Figure 2.3: Menu Screen developed by Dr. Reynolds and Khalid Kattan........................... 38

Figure 2.4: Displays the Database diagram with two index variables, fish and location ID .. 39

Figure 2.5: Gives the Catch Log screen result for catch ID 1...................................... 40

Figure 2.6: Gives the Fish Maintenance Screen. .................................................... 41

Figure 2.7: This screen shows the Location Maintenance screen..................................... 42

Figure 2.8: This screen shows the Ad-hoc Reporting................................................. 43

Figure 2.9: The Reporting by Species Type and Location Type. .................................... 44

Figure 3.1: The Total catch count per month relative to trip direction for: Lorna................ 52

Figure 3.2: The Total trip count per month relative to trip direction for Lorna................... 53

Figure 3.2: The Trip Count by Species Type for the Entire Period .................................... 66 
Figure 4.1: The Framework of Cultural Algorithm [21]............................................. 69

Figure 4.2: Pseudo code for Cultural Algorithms [14] ................................................ 70

Figure 4.3: Knowledge Update in the Belief Space [23] ............................................... 75

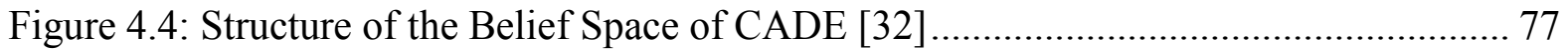

Figure 4.5: Example of the Roulette Wheel Function [30].......................................... 78

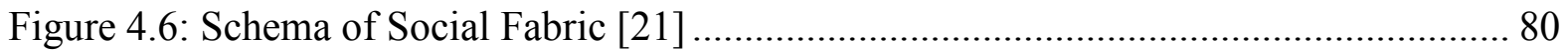

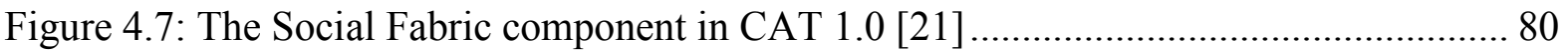

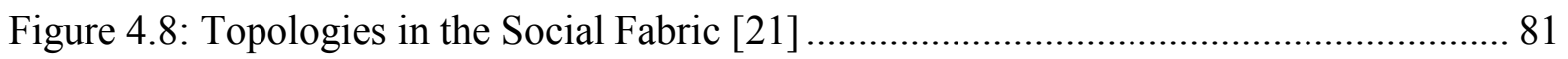

Figure 4.9: Knowledge Source Interaction by [23] ................................................ 83

Figure 4.10: Majority Win in Belief Space by [23] ..................................................... 84

Figure 4.11: Weighted-Majority Win in Belief Space by [23] ...................................... 84

Figure 4.12: Different Knowledge Distribution Mechanisms. ......................................... 86

Figure 5.1: Artisanal fisherman - traditional techniques [37] ...................................... 93

Figure 5.2: The Pareto curve is the set of non-dominated points [38] .............................. 95

Figure 5.3: An example Pareto Front for Payout and Effort............................................ 96

Figure 5.4: A Decision Tree of the sample tour of the first 8 days using $(0,100) \ldots \ldots \ldots \ldots . . . .102$

Figure 5.5: Details about the Decision Tree of the sample in Figure 5.4 ......................... 102

Figure 6.1: Pareto Front for Payout, Effort, FK Phase I- EM......................................... 106

Figure 6.2: A plot of 500 tours generated for the Pareto Front - Phase I Full Wk .............. 107

Figure 6.3: Plot of 500 Tours that contain No Sundays for fishing agents in Phase I......... 109

Figure 6.4: The two Pareto Fronts for Payout, Effort, Phase I, No Sundays ...................... 110

Figure 6.5: Plot of 500 points of the values $M_{-} T_{-}$W Phase I fishermen............................ 112

Figure 6.6: Pareto Front for Payout, Effort, Mon_Tue_Wed Phase I................................ 113 
Figure 6.7: Plot of 500 points of the real values RFS Phase I 114

Figure 6.8 Pareto Front for Payout, Effort, Thur_Fri_Sat Phase I.................................... 116

Figure 6.9: Plot of 500 points of randomly generated tour values for Full Wk in Phase II.. 118

Figure 6.10: The dominant Pareto Front for Payout, Effort, Full week Phase II................. 119

Figure 6.11: Plot of 500 tours for No Sundays in Phase II.......................................... 120

Figure 6.12: The Pareto Front for Payout, Effort for tours with No Sundays. Phase II ....... 121

Figure 6.13: Plot of 500 randomly generated tours for just those that use MTW, Phase II. 122

Figure 6.14: The Pareto Front for Payout vs. Effort for tours on MTW Phase II................ 124

Figure 6.15: The Plot of 500 points for trips starting on Thur_Fri_Sat in Phase II............. 125

Figure 6.16: The Dominant Pareto Front for Payout, Effort, Thur_Fri_Sat Phase II.......... 127

Figure 6.17: The Plot of 500 randomly generated tour values for Full Week in Phase III... 129

Figure 6.18: The dominant Pareto Front for Payout vs. Effort for Full week in Phase III... 130

Figure 6.19: The Plot of 500 points of tour values that exclude Sundays in Phase III........ 131

Figure 6.20: The Pareto Front for Payout vs. Effort for No Sundays.............................. 132

Figure 6.21: Plot of 500 randomly generated tours for MTW in Phase III. ....................... 133

Figure 6.22: The Pareto Front for Payout vs. Effort for just MTW tours in Phase III. ........ 134

Figure 6.23: The Plot of 500 tour values for RFS tours in Phase III. ............................... 135

Figure 6.24: The Pareto Front for Payout vs. Effort in TFS Phase III............................... 136

Figure 7.1: Curve fitting for Payout, Effort for Full week Phase I................................. 141

Figure 7.2: Scattering points for Residuals for Full week Phase I................................. 141

Figure 7.3: Curve fitting for Payout, Effort for No Sundays Phase I .............................. 142

Figure 7.4: Scattering points for Residuals for No Sundays Phase I .............................. 142

Figure 7.5: Curve fitting for Payout, Effort for $1^{\text {st }}$ half of the week Phase I ..................... 143 
Figure 7.6: Scattering points for Residuals, $1^{\text {st }}$ half of the week Phase I 143

Figure 7.7: Curve fitting for Payout, Effort, $2^{\text {nd }}$ half of the week Phase I....... 144

Figure 7.8: Scattering points for Residuals, $2^{\text {nd }}$ half of the week Phase I 144

Figure 7.9: Curve fitting for Payout, Effort, for Full Week Phase II.................................... 146

Figure 7.10: Scattering points for Residuals for Full week Phase II ................................... 146

Figure 7.11: Curve fitting for Payout, Effort for No Sundays Phase II ................................ 147

Figure 7.12: Scattering points for Residuals for No Sundays Phase I................................ 147

Figure 7.13: Curve fitting for Payout, Effort for MTW Phase II.......................................... 148

Figure 7.14: Scattering points for Residuals for MTW Phase II ........................................ 148

Figure 7.15: Curve fitting for Payout, Effort for TFS Phase II........................................... 149

Figure 7.16: Scattering points for Residuals for TFS Phase II .......................................... 149

Figure 7.17: Curve fitting for Payout, Effort for Full week Phase III. ................................ 152

Figure 7.18: Scattering points for Residuals for Full Week for Phase III ............................. 152

Figure 7.19 Curve fitting for Payout, Effort for NS Phase III............................................ 153

Figure 7.20: Scattering points for Residuals for No Sundays Phase III. .............................. 153

Figure 7.21: Curve fitting for Payout, Effort for MTW Phase III. ....................................... 154

Figure 7.22: Scattering points for Residuals for MTW Phase III …………………............ 154

Figure 7.23: Curve fitting for Payout, Effort for RFS Phase III........................................... 155

Figure 7.24: Scattering points for Residuals for RFS Phase III........................................... 155

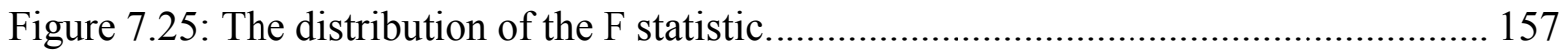




\section{CHAPTER 1: INTRODUCTION AND MOTIVATION}

\subsection{INTRODUCTION}

The site of Cerro Azul has been occupied for hundreds of years under a variety of different socio-political structures [1]. While the socio-political context has changed over time, the basic economics of the area is still influenced by the pattern of offshore fishing. In the 80 's, when excavating an early site associated with the current location of modern Cerro Azul, Dr. Joyce Marcus arranged to have results of local fishing expeditions tabulated in terms of the catches returned from various nearby locations.

Although the technology for artisanal local fishing had changed in many ways, the fishermen were limited to harvesting catches reasonably close to shore rather than deeper at sea. They were also still dependent on the unpredictability of the local climate and the food chains that it supported [1]. In fact, during the period of study, fishermen were observed to respond to an El Nino. This El Nino disrupted the traditional oceanic food chain and may have impacted fishermen decision making. While El Nino brought warmer waters to the area bringing with them invasive species, it was followed by a La Nina where the colder waters returned, gradually bringing back the normal food chains that fishermen had been accustomed to. This followed by the start of a return to normal relative to the aquatic environment [1].

Clearly this was not the first, nor will it be the last El Nino to affect Cerro Azul. Additionally, while there has been considerable discussion of the impact of climate change in its many forms on the global arena, little research has been done on the impact on the local economy of an area. As a result, the goal here was to use the raw data collected by Dr. Marcus as the basis for a model of fishermen decision making in response to each of three 
successive phases related to the El Nino event: residual El Nino, La Nina, and the return to normal phase. The main hypothesis of this work is that local fishermen have adopted a sequence of decision-making behaviors over time in order to facilitate the sustainability of the local economy in light of these environmental perturbations. In order to do this, we will utilize models having cultural adaptation, for example, Cultural Algorithms. Cultural Algorithms have been shown to be effective measures of collective intelligence [2].

To accurately model any complex system, we must have the necessary data. Dr. Marcus has provided a data set that contained information about all offshore fishing expeditions at Cerro Azul between the dates of March 1, 1984 through July 28, 1986 [1]. This dataset is comprised of 6013 records capturing fishing expeditions based upon trips taken out of Cerro Azul harbor and reported upon return to the local official present at the site. Catches produced by other methods, such as fishing in deeper water farther off shore were not considered. In addition, catches were only recorded when a local official was on duty. Also, the focus on the major catch produced per trip was reported.

Even with these limitations, the data set represents a major opportunity to investigate the impact that climate change has on the millions of local environments around the world. However, in order to do this, we need a model of cultural change or evolution that is capable of characterizing how individuals adapt to each of these scenarios. The model that we chose to use is Cultural Algorithms developed by [2]. It was derived from the models of Kent Flannery on Cultural Evolution [3] . Cultural Algorithms have been shown to be a robust vehicle for the describing the evolution of complex social systems. It has been used to model the origins of agriculture [4], the Origin of the State [5] the Collapse of Anasazi Culture [6], Peruvian Llama herding adaptations [3], and Prehistoric Artic Hunting behavior. We begin 
our journey in the next section with a discussion of the environmental setting for the evolutionary model.

\subsection{THE CERRO AZUL ECOSYSTEM}

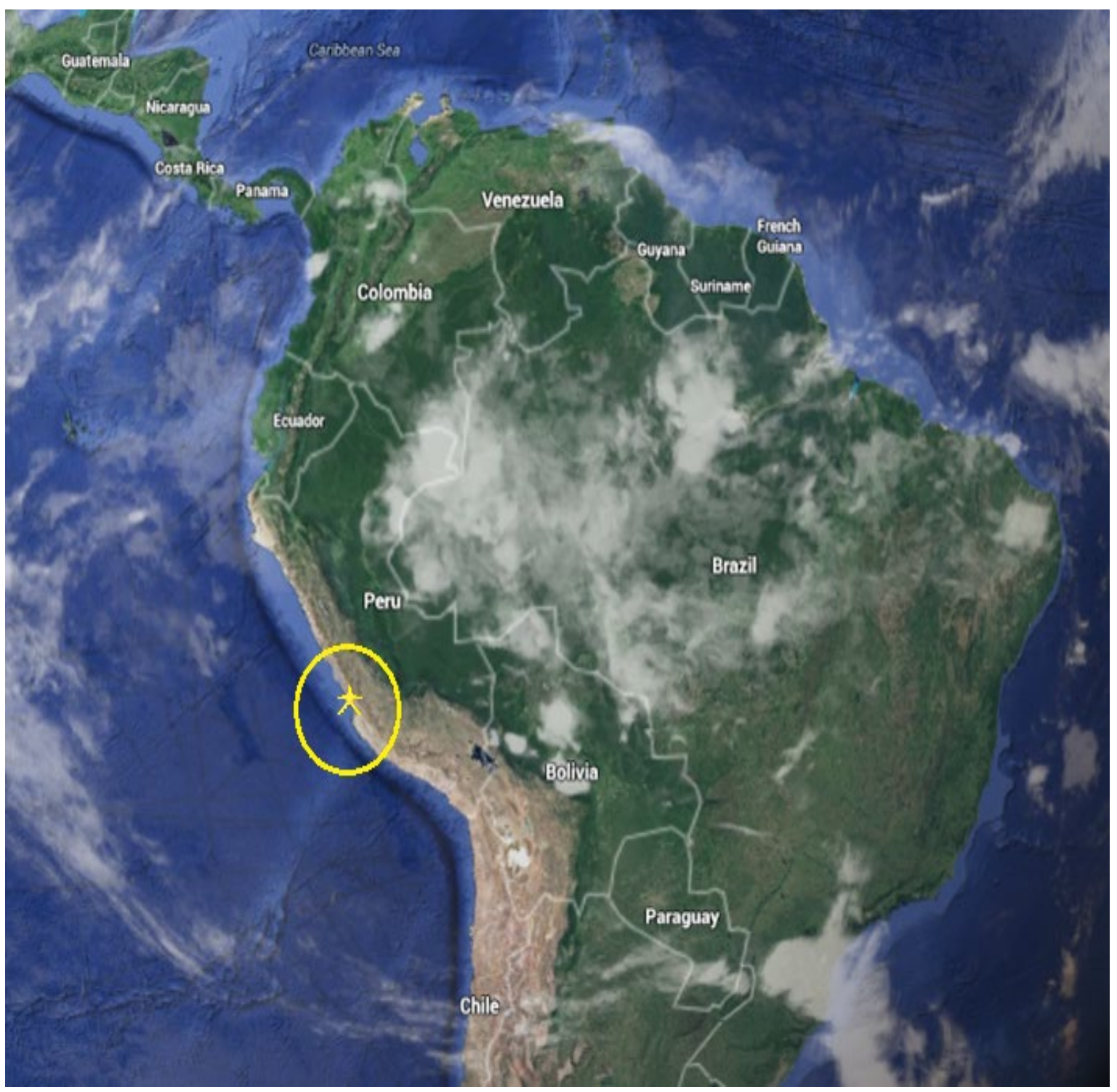

Figure 1.1: The site location of Cerro Azul location, Yellow Star inside Yellow Circle. 


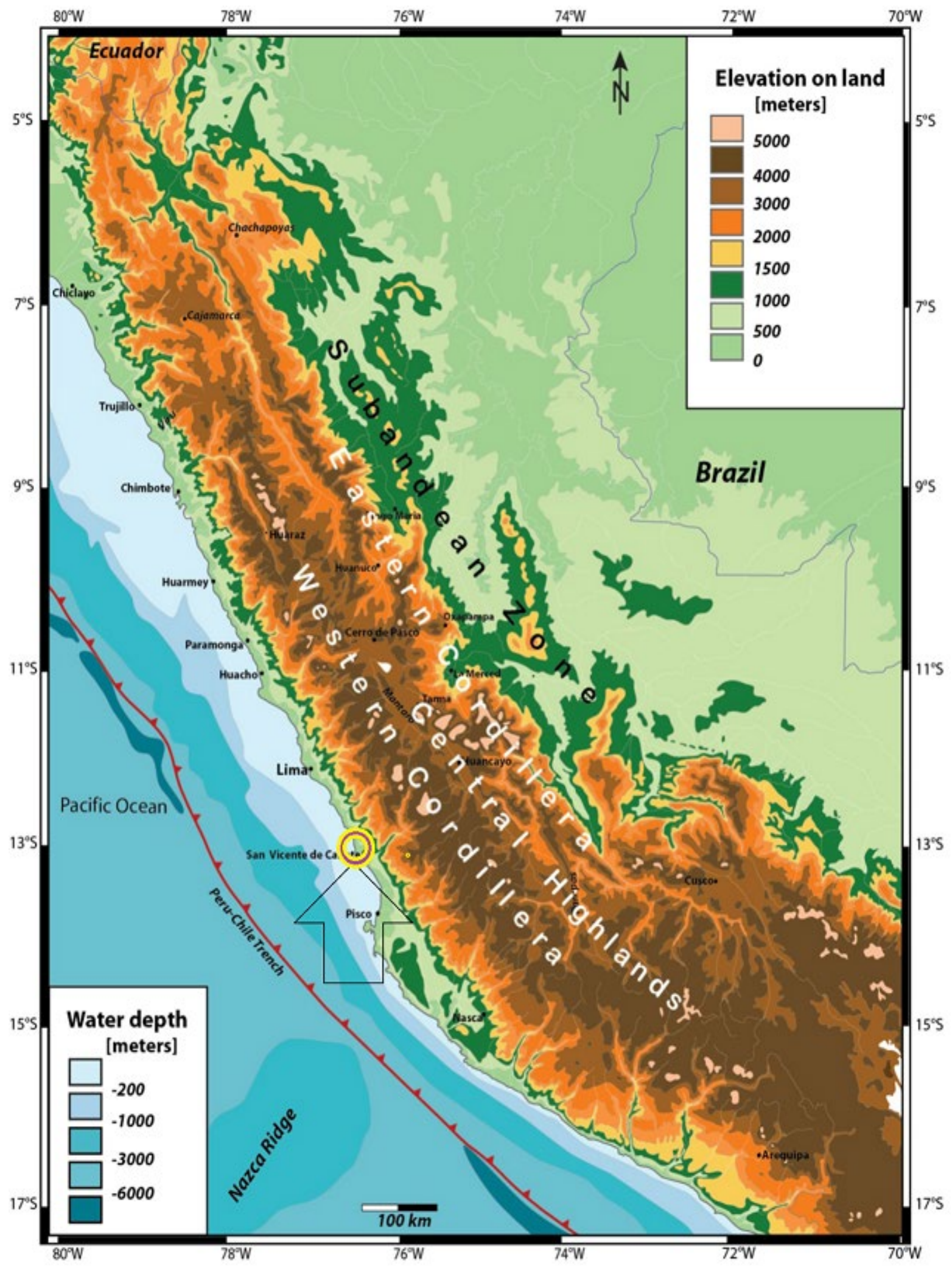

Figure 1.2: The Topographical Map of Peru. Cerro Azul is in the yellow circle. 
The town of Cerro Azul is located south of Lima, Peru on the Pacific Ocean as shown in Figure 1.1. It is part of the Canete Valley [7] and its location is highlighted in the topographic map shown in Figure 1.2. The coastal area is comprised of three basic geologic formations: the playa or sandy beach just north of Cerro Azul bay; cliffs of volcanic rock to the immediate south; and a narrow band of cobbled shore that extends south to the Rio Canete. This geological diversity was certainly a factor in the creation of a diverse collection of aquatic resources. Figure 1.3 gives the relative location of the modern town of Cerro Azul and the ancient site [1]. The ancient site is displayed with contour mapping. The area in front of the modern town is composed of sandy beach or playa. The darkened coastline to the south represents the volcanic cliff that extends to the Centinel or lighthouse. After that the coastline is a narrow band of pebbles and rocks. 


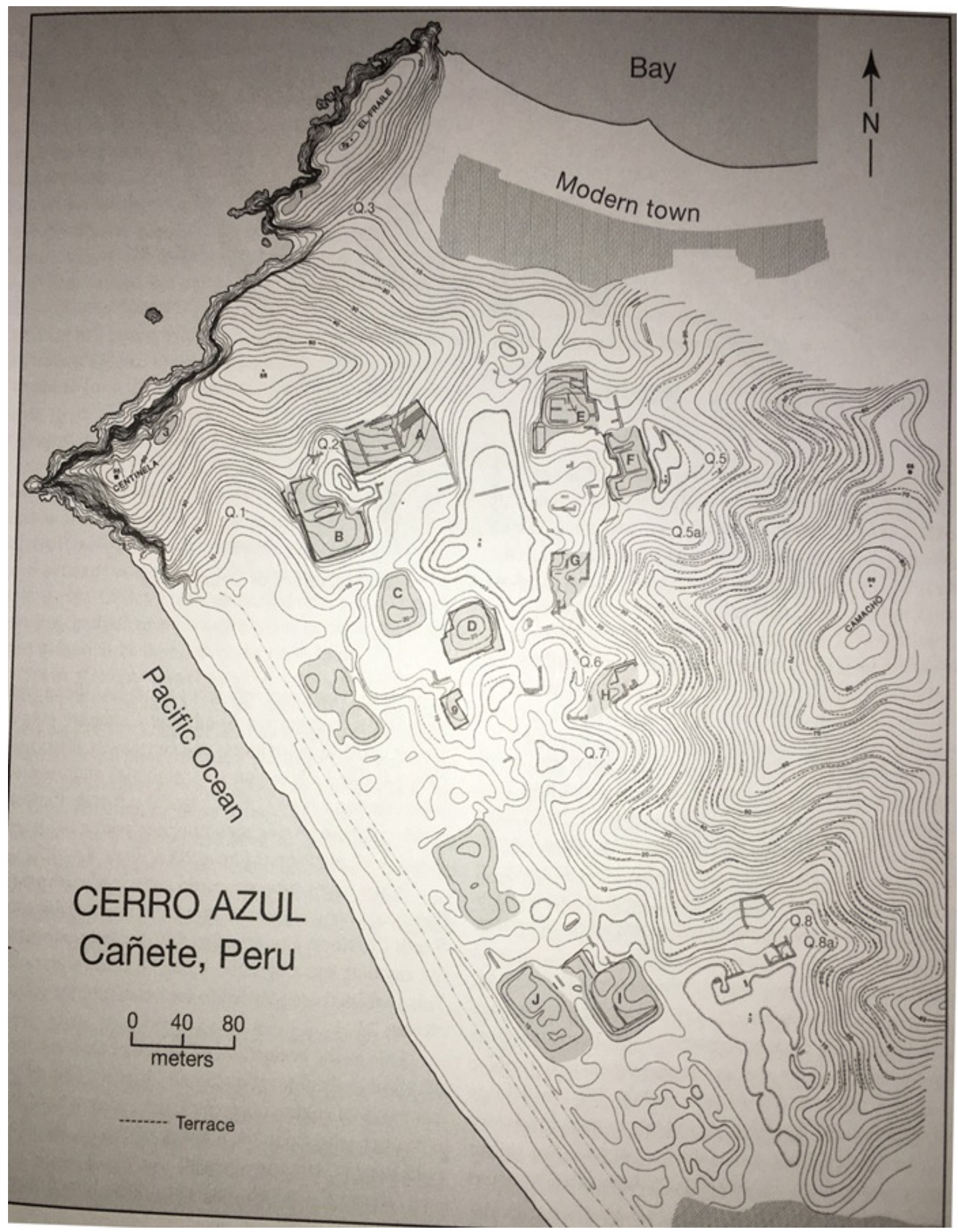

Figure 1.3: Relative location of modern day and prehistoric Cerro Azul [1] 
Over 50 species were caught during the period of the survey, and evidence for 30 or more came from the nearby archaeological site of Cerro Azul. Most of these are found in the intertidal zones and shallow waters just off of the coast. The difference in those harvested in ancient and modern times can be attributed to changes in technology (nets, motors, etc.) and the changing dynamics of the marine ecosystem. The geologic diversity has resulted in a subsequently diverse food chain with four basic tropic levels. Level One consists of energy producers, algae and small phytoplankton. They convert energy from the coastal waters into plant-based energy. They are consumed by small animals that comprise levels two and three in the aquatic food chain. They predators include zooplankton, various crustaceans, mollusks, as well as sardines and anchovies. Level three is characterized by medium level predators such as octopi, squid, fish such as hake, along with sea lions, and various and diverse bird species. The top level of the food chain, level 4 , is comprised of sharks, large squids, whales, and flounder.

Cerro Azul is located in a subtropical desert zone and the number of indigenous species is relatively sparse. However, with the introduction of irrigation canals the number of plant species has increased markedly. The principle crops are cotton, potatoes, and maize. Cotton is planted in the spring (November) and harvested in the fall, while potatoes are planted in the fall and harvested in the spring (November). Maize is grown over the entire year.

Figure 1.4 taken from [1] gives an illustration of how these different environmental components interact to produce a complex system. The sketch describes how the marine and land-based economies interact through the actions of human agents at the nearby ancient site of Cerro Azul in the Late Intermediate Period (1000 A.D - 1470). Energy moves up the food 
chain from producers to consumers in the marine food chain and is harvested by medium predators, birds. The birds transfer the energy into guano that is then used by human to fertilize the irrigated fields. Produce from the fields are then used to provide the fishermen with resources to harvest the ocean species such as anchovies and sardines. The excess harvest can then be dried and shipped to more inland places.

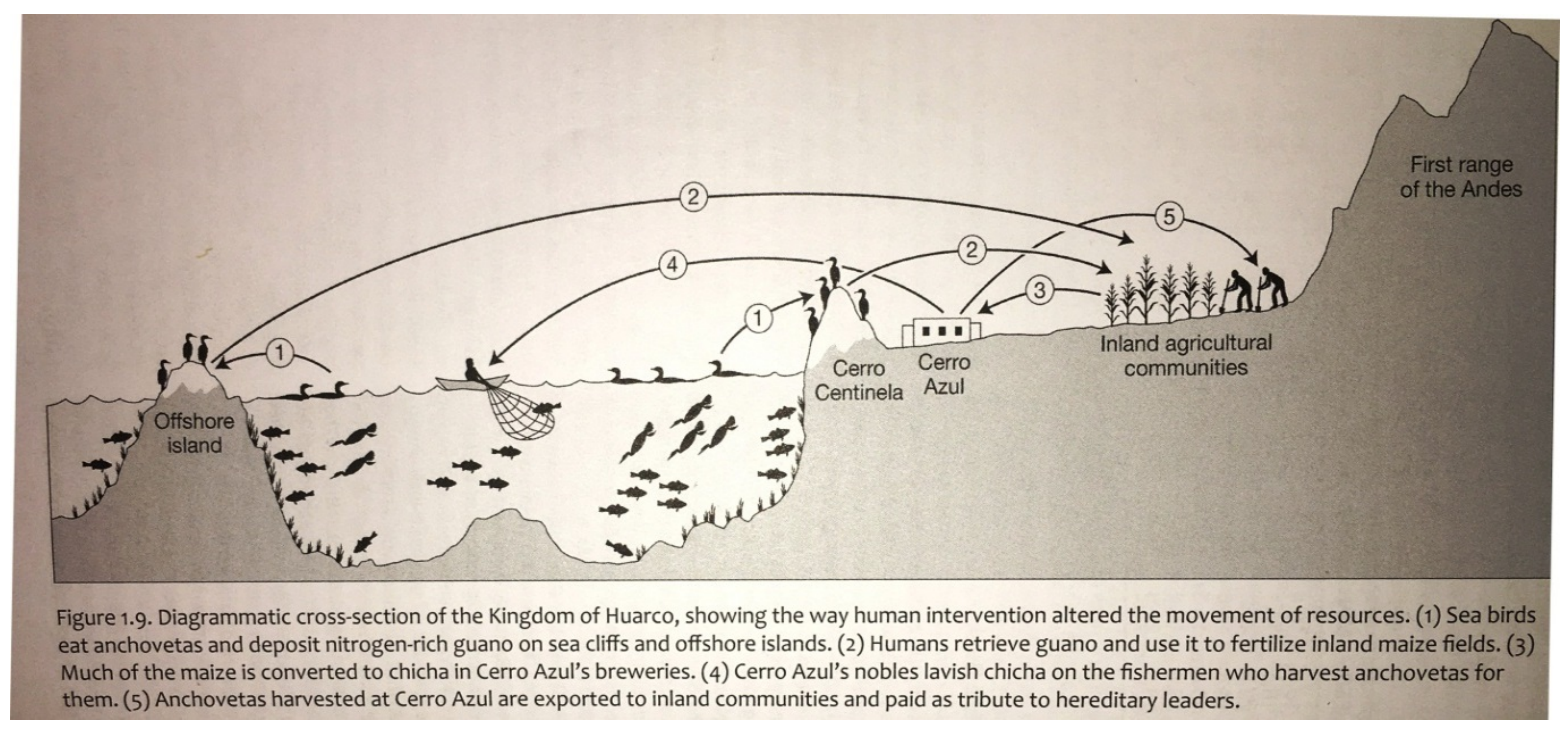

Figure 1.4: Interactions between environmental components [1]

The nature and extent of these interactions have changed over the years, but clearly the environment can be described as a complex system. A complex system is characterized by the nonlinear interaction of its components, such that a small change in one component can produce major changes in other components through feedback loops. Such changes can result in the new adaptations, and the emergence of spontaneous organization of components at different levels of granularity [8]. Even a relatively slight change in ocean temperature can cause non-trivial adaptations or adjustments in the interactions with the other components. In the next section, we look at a particular source of short term change, the El Nino-Southern Oscillation (ENSO). An ENSO cycle is composed of a year of above average water 
temperatures (El Nino) followed by a period of cooling temperatures (La Nina). In this work we will be concerned with modeling adaptations of local offshore fisherman to the changes produced as the result of a larger than normal ENSO cycle.

\subsection{ENSO: AN EXAMPLE OF SHORT TERM CLIMATE CHANGE.}

The El-Nino portion of the El-Nino Southern phenomenon was discovered in the 1600's by Peruvian fisherman. In a typical year the east-to-west rotation of the earth produces trade winds that collect heat and moisture off of the coastal regions of Peru and blow them west towards Indonesia. This allows an upwelling of the cool Humboldt Current to dominate the regions along the continental shelf [1]. This is shown schematically in Figure 1.5 .

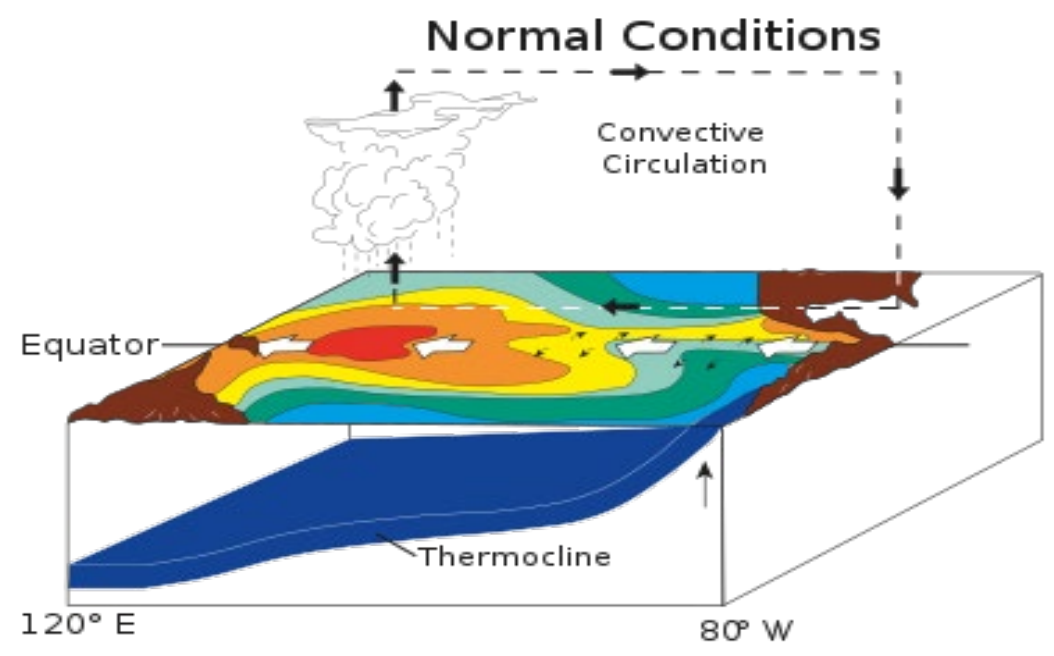

Figure 1.5: Normal conditions for circulation of the heat. [9]

Notice that warmer waters move westward towards Indonesia and create a thermocline or upwelling of cooler waters at 80 degrees west nears the coast of Peru. This causes a corresponding circulation of air in the opposite direction towards the Peruvian coast as shown in Figure 1.5. This produces abundant rainfall in Indonesia that benefits the rice 
crops there. On the other hand, it produces a desert like dry area along the South American Coast.

Occasionally, certain conditions in the atmosphere lead to the reduction in the strength of the trade winds which means that less warm water is moved from the east (South America) to the West (Indonesia). This reduces the gradation of the thermocline and leads to a warming of water on the South American side as shown in Figure 1.6. This produces dry weather and crop reductions in Indonesia and heavy rainfall and corresponding floods along the South American coast. At the same time the warming temperature of the South American waters kills phytoplankton and algae and forces the fish that normally feed on them off the coast to move elsewhere. Warm water fish then move south to fill in the gaps left by the departure of the cold water variety.

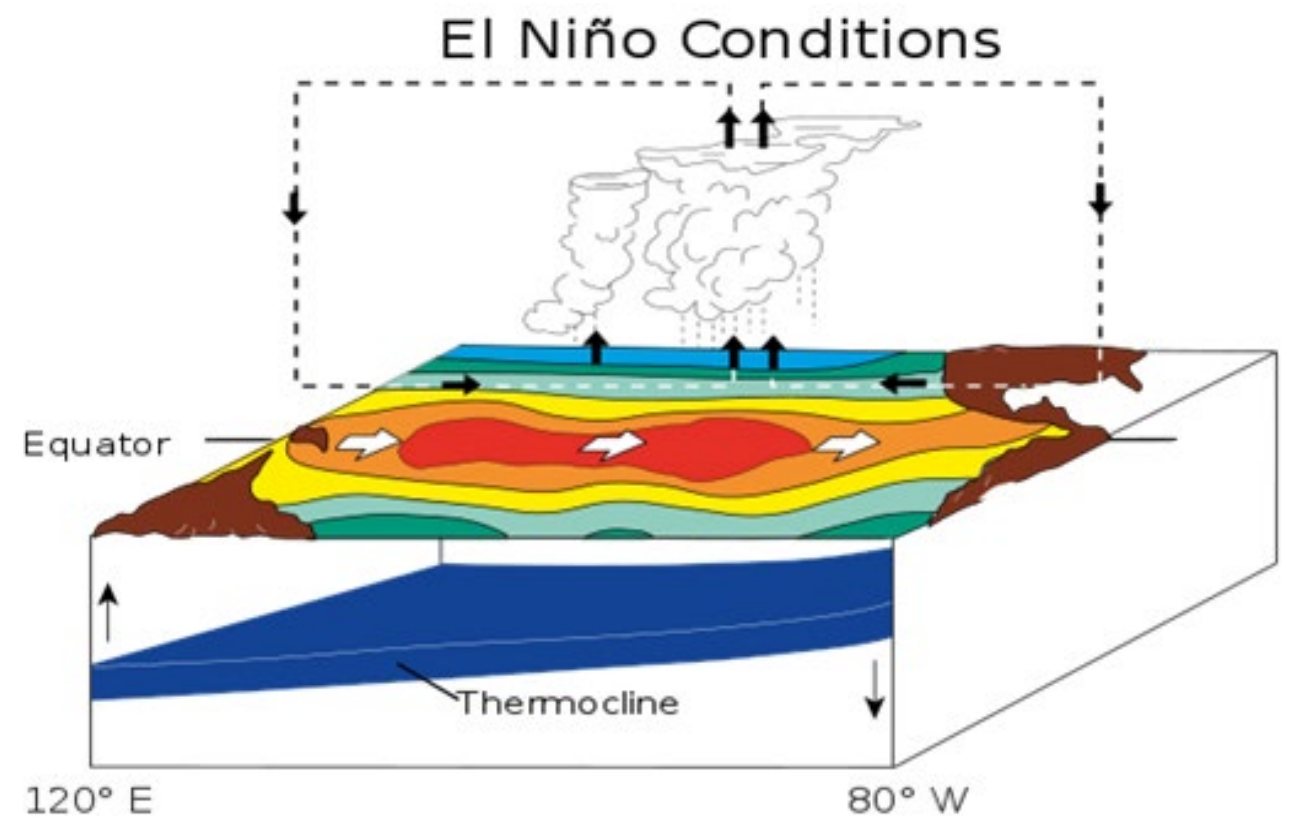

Figure 1.6: The conditions produced by an El Nino. [9] 
The technical definition of El Niño is characterized by a positive ONI (Oceanic Nino Index) where equatorial sea surface temperatures are greater than or equal to $+0.5^{\circ} \mathrm{C}$. $\mathrm{La}$ Niña is characterized by a negative ONI, less than or equal to $-0.5^{\circ} \mathrm{C}$. For a full-fledged El Niño or La Niña episode, these thresholds must be exceeded for a period of at least 5 consecutive overlapping 3-month seasons [9]. Although 0.5 degrees Celsius might not seem much locally, this is an overall elevation in an ocean with an enormous amount of volume. Additionally, some species are sensitive to very minor changes in water temperature. These changes in water temperature undoubtedly will affect the distribution of species and the potential disruption of natural food chains as well. Figures 1.7 shows the surface temperature gradient over the Pacific Equatorial region from South America to Indonesia produces as a result of the El Nino [10].

Figure 1.8 show monthly ocean temperatures at Callao, Peru near where Dr. Marcus collected her data, between 1981 and 1986 [10]. Notice the huge spike starting in August 1982 when the temperature began at 15.9 degrees Celsius and peaked at 24.2 degrees Celsius, an increase of 8.3 degrees Celsius, during the very powerful El Nino of 1983. Then there is a subsequent cooling phase, La Nina, where the water cools back down to normal oceanic temperatures and the thermocline and trade winds readjust accordingly. The cooling effects of the La Nina are displayed in Figure 1.9.

The impact on the past and present sites of Cerro Azul has been described by [1]. The data collected during her period of observation indicates that a number of invasive warm water species moved into the area to harvest increased population of invertebrates. These invasive species include invertebrates. [12] monitored small animal life on the floor of Ancon Bay from 1981 to 1984. During the 1982 -1983 El Nino, the episodes of short-term warming 
increased the Oxygen saturation of this bay's deep-water zone from $<8.5 \%$ to $>20 \%$. As a result, the small mollusk species increased from 0 to 5 ; the small Crustacean species rose from 0 to 10; and the species of bristle worms rose from 4 to 29 [1].

As late as March 1984, the recorded data shows that fishermen of Cerro Azul were still catching shark Paloma, Pompano, the Pez Dama or whale shark, Dolphin. Then came two mild La Nina episodes, the episodes of short-term cooling and the invasive tropical species were gone. Their place was taken by the Lorna and the Mismis, both of which flourished under the cooler conditions of La Nina. Dr. Marcus found no remains at Cerro Azul of invasive species such as shrimp, pompano, dolphin, or arched blue crab, or shrimp. These are four species that are likely to have the area in El Nino years.

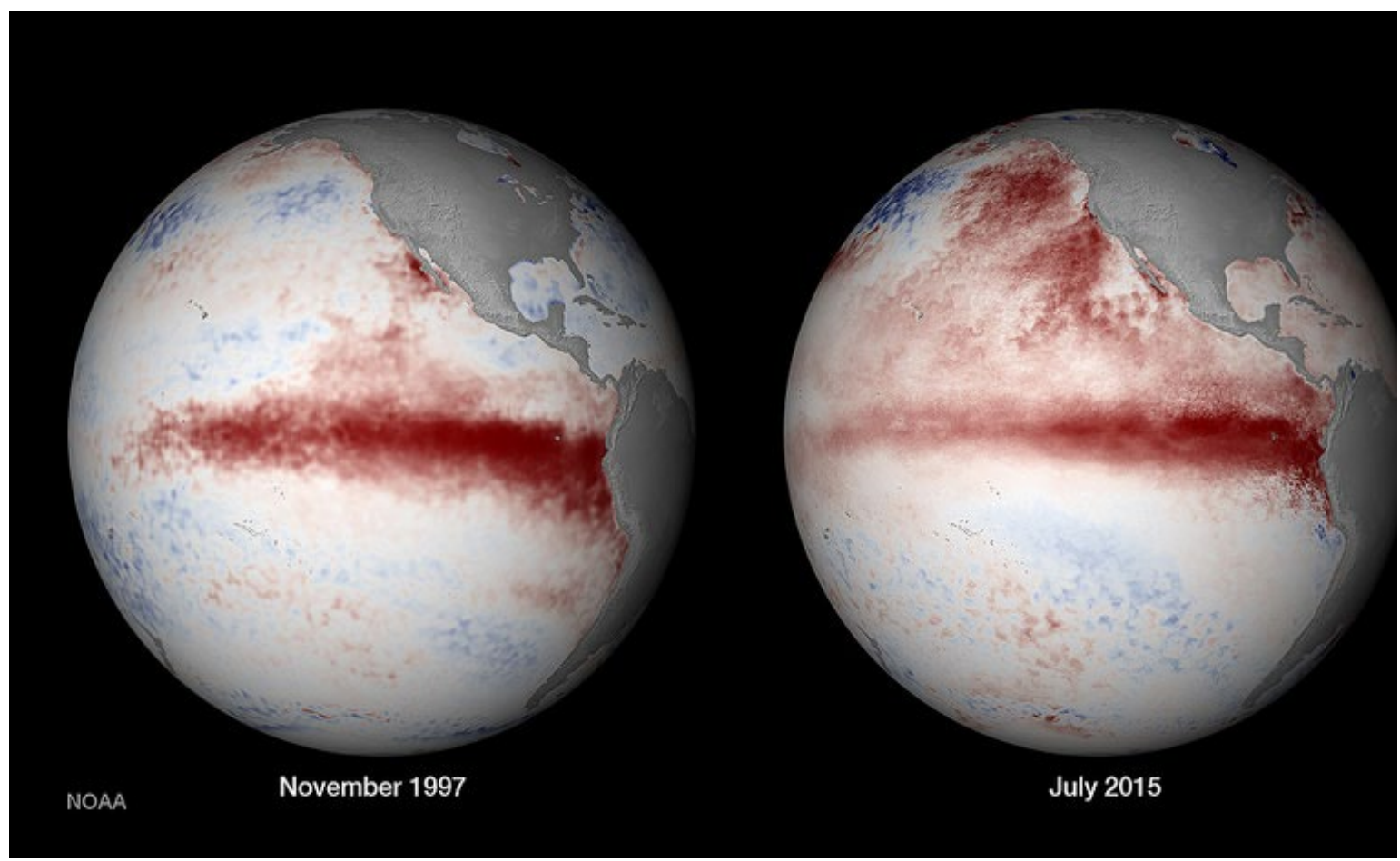

Figure 1.7: A heat map comparing the powerful El-Nino of 1997 to 2015 


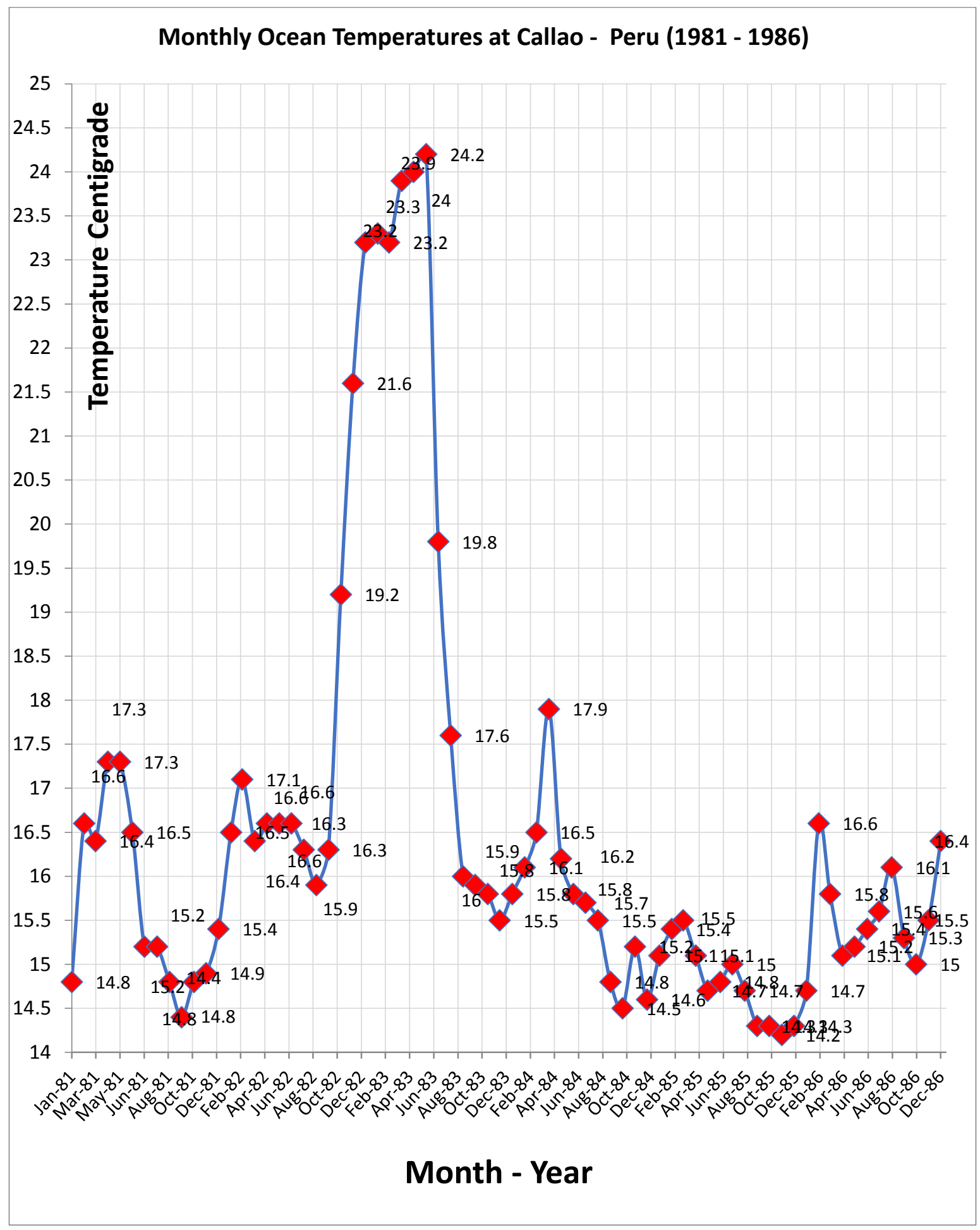

Figure 1.8: Monthly avg. temperatures at Callu, Peru from 1981 to 1986 [1] 


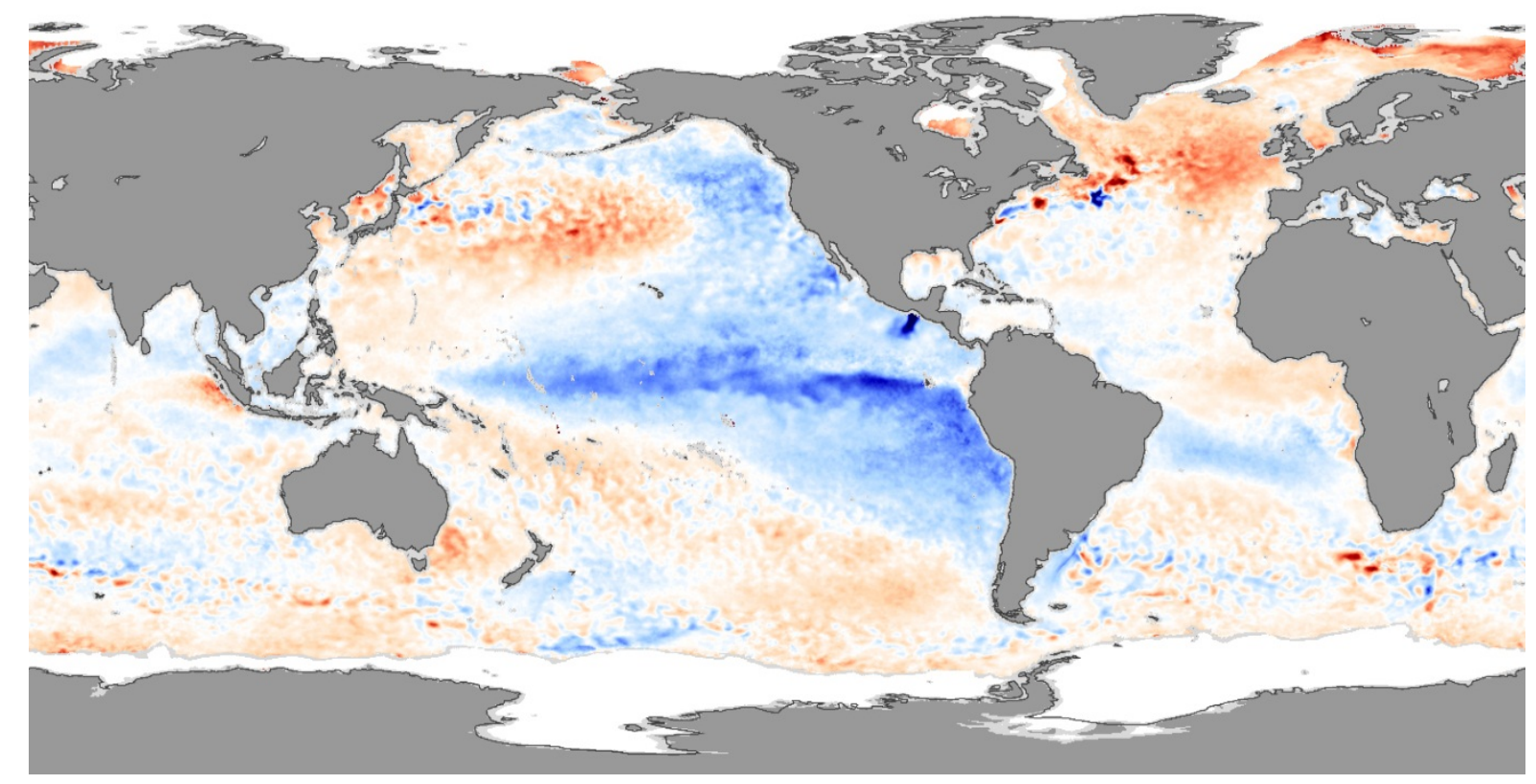

Figure 1.9: La Nina and its cooling affect in blue.

While the intensity for each of these El Nino occurrences varies, Dr. Marcus' data was collected during a very powerful El Nino [13] and includes almost 50 different catch types collected from over 30 different site locations. The focus of this thesis will be to use tools from the area of Collective Intelligence, Cultural Algorithms, to assess the adaptation the modern fishermen have had to make in order to adjust to the sequence of changes produced as a result of the ENSO process. It is anticipated that an understanding of these adjustments will lead to a better understanding of what was done in ancient occupation such as that of Huarco.

\subsection{CULTURAL ALGORITHMS}

Classical optimization problems are more efficient than evolutionary algorithms in the solution of linear, quadratic, and strongly convex algorithms. However, evolutionary algorithms have been shown to be more efficient for discontinuous, non-differentiable, multimodal and noisy problems. The main difference between these two approaches is how they 
implement their search process and how they utilize their information about the search space. Classical optimization problems use deterministic rules to move from one point to another in the search space. Evolutionary Algorithms, in contrast, use probabilistic, non-deterministic, transition rules. In addition, classical optimization uses sequential search while evolutionary computation implements parallel search. Different classes of Evolutionary Algorithms (EA) have been developed that are based upon models of natural evolution. These nature-inspired approaches include Genetic Algorithms, Genetic Programming, Evolutionary Programming, Evolution Strategies, Particle Swarm Optimization, Ant Colony Optimization, Cultural and Co-evolutionary Algorithms among others, [13].

It has been shown that if Evolutionary Algorithms were to utilize domain knowledge to make decisions, the overall system performance can be increased. Domain knowledge can reduce the search space by heuristically by pruning undesirable parts. In 1979, Reynolds incorporated the domain knowledge explicitly into the evolutionary process with the introduction of Cultural Algorithms [14]. Cultural Algorithms, CAs, model the principles of human social evolution, and can bias the search process with prior knowledge about the domain. Also, Cultural Algorithms can utilize information gained during the evolutionary process. Cultural Algorithms can be viewed as a direct extension of Genetic Algorithms with the additional component of the belief space to explicitly hold information collected by an evolving population over time [2].

Cultural Algorithms are a model of collective intelligence in which groups of individuals are able to interact with each other to solve problems that no individual is able to do on its own. The majority of these models are inspired by real-life, naturally occurring systems. These include Particle Swarm Optimization (PSO), Ant Colony Optimization 
(ACO), and Cultural Algorithms (CA) [13]. These algorithms are different in the way they process and exchange information over the spatial and temporal scale as represented in Figure 1.10. The scales grow exponentially from particle swarms, ant, chimps and hominids, and then finally to Cultural Algorithms as stated by [14] In terms of spatial and temporal scale, Cultural Algorithms encompass all the other algorithms, so we will focus on the performance of socially motivated systems from the standpoint of Cultural Algorithms in this thesis.

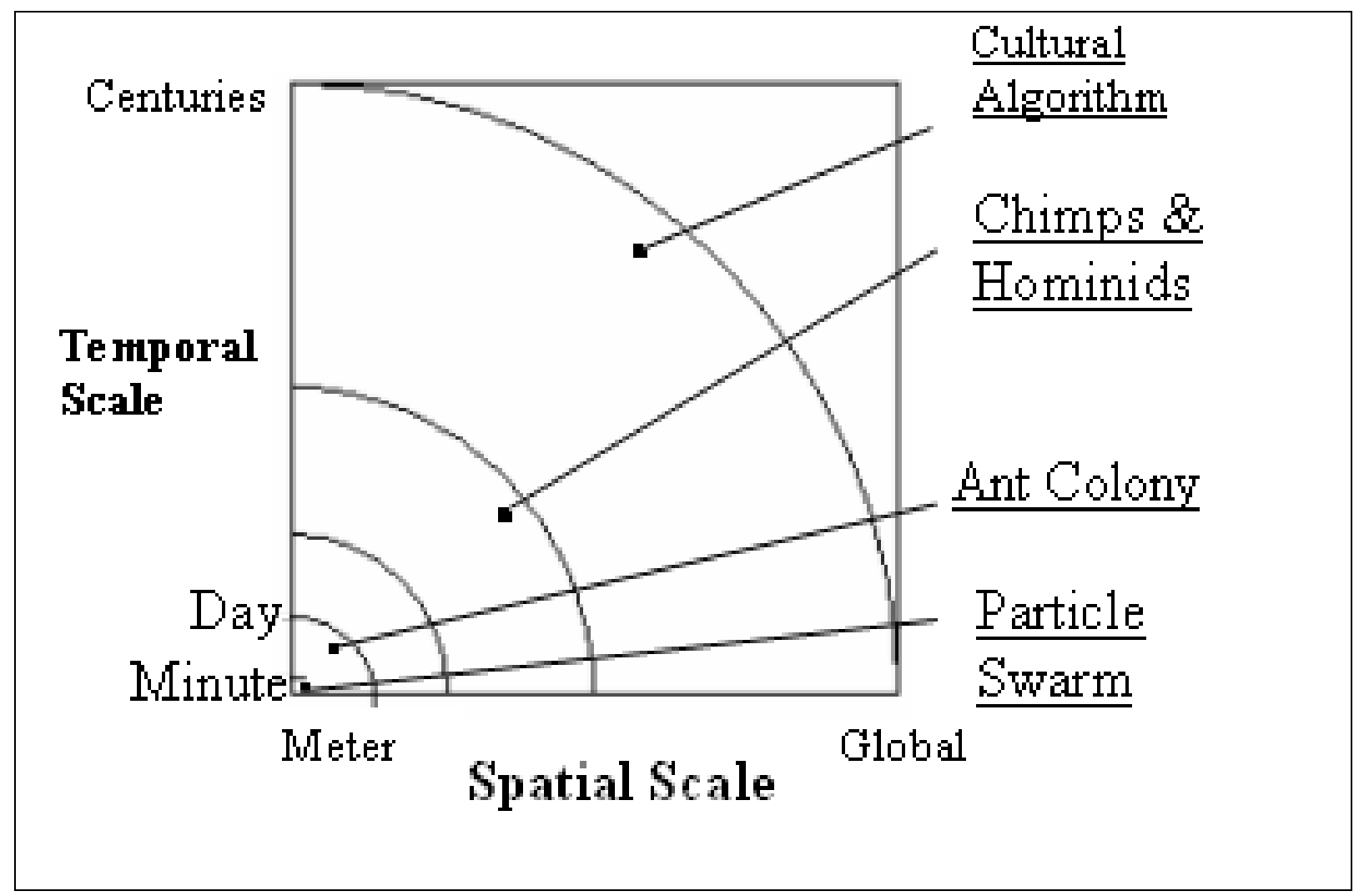

Figure 1.10: The Scale of Social Interaction [14]

The Cultural Algorithm portrays evolution in human culture at both the macroevolutionary level, which takes place within the belief space, and at the micro-evolutionary level which occurs in the population space. As such, it can be viewed as a co-evolutionary 
mechanism that supports dual inheritance of knowledge in both the Belief Space and the Population Space. The two co-evolutionary components are connected by a communication protocol that allows the adaptations in each of the two components to influence each other.

What differentiates Cultural Algorithms from existing evolutionary algorithms is that Cultural Algorithms can use different knowledge sources in the problem-solving process instead of just one or two locally transmitted value. The study of Semiotics has shown that various types of knowledge can be stored and retrieved by different species in nature, often influenced by their specific niches. The knowledge sources include normative knowledge (ranges of acceptable behaviors), situational knowledge (exemplars or memories of successful and unsuccessful solutions etc.), domain knowledge (knowledge of domain objects, their relationships, and interactions), history knowledge (temporal patterns of behavior), and topographical knowledge (spatial patterns of behavior). This set of categories is viewed as being complete for a given domain in the sense that all available knowledge can be expressed in terms of a combination of one of these classifications. These knowledge sources can be configured as a network in the Belief Space such that changes in one knowledge source can impact changes in another over time.

The population space is characterized by a network of agents that connects with each other in terms of a social network. The network is termed a Social Fabric because the network connections can be dynamically adjusted over time based upon use. Individuals can distribute knowledge through the network by a variety of mechanisms including majority win, auctions, and games. 


\subsection{MULTI-OBJECTIVE PROBLEM STATEMENT}

In this research we investigate the potential for building agent-based models of fishing patterns by applying a suite of tools from Artificial Intelligence and Data Mining to existing archaeological data from the fishing port, Cerro Azul, Peru. Perhaps we can discover some behavior tendencies between the fishermen. Additionally, an interesting question is what impact the ENSO has on fishing patterns at the site over time.

The key to our approach here is that decision making for fishermen can take the form of two complementary objectives.

1) One objective is to optimize the number of high-quality catches, those that will be attractive to consumers.

2) A second goal is to minimize the amount of resources consumed in the process of the fishing activity. In other words, they wish to optimize their ability to just be able to fish regardless of what is caught.

We can model the interaction between these two goals as a competitive game. Evolutionary techniques have been successfully employed to model the trade-off between conflicting goals, multi-objective optimization. A specific version of Cultural Algorithms has been developed to solve knowledge intensive multi-objective problems, the Multi-Objective Cultural Algorithms Toolkit (MOCAT), [15]. We will use the MOCAT system here to model the tradeoffs between these goals as they are impacted by the changing environment. These tradeoffs will be represented mathematically via a Pareto front, where optimum combinations of these goals are viewed in terms of tradeoffs in resources. MOCAT will be discussed in subsequent chapters. 


\subsection{ORGANIZATION OF THE DISSERTATION}

Chapters 2 through 3 focus on the data and statistical basis for the model to be developed. In chapter 2, we present the database of records produced from the survey conducted by Marcus. It contains a discussion of the database of fishing trips that will be used as the basis for the construction of the fishermen decision-making model. Each entry in the database will be associated with one of the three phases of the ENSO observed here: the residual El Nino; the La Nina or cooling phase; and the back to normal phase when temperatures have returned to normal. Chapter 3 provides statistics about fishing behavior at three different scales of analysis, macro, meso, and micro, for each of these three phases. The macro level provides analytics that summarize behavior over the entire period of observation. The meso scale corresponds to monthly patterns of behavior. Finally, the Micro scale provides statistics about fishing behavior on a daily basis.

Chapters 4 through 5 are dedicated to model development. Chapter 4 gives an overview of Cultural Algorithms. It describes the basic Cultural Algorithms framework and demonstrates how that can be used for the solution of single CAT (Cultural Algorithm Toolkit) and multi-objective problems. Chapter 5 gives an overview of the Multi-Agent fishing model employed here. Chapters 6 through 7 provide results for the multi-objective analysis used here. It is shown that there appears to be a daily learning curve with regards to where and when fishing trips should be taken; and, that the nature of these trips differs between the El Nino, La Nina, and Back to Normal phases. These differences represent decision-making adaptations to the changes in the environment produces by the ENSO. It is shown that these differences are statistically significant and represent the adjustment of the 
collective consciousness to the observed environmental events. Chapter 8 gives our conclusions. 


\section{CHAPTER 2: AN OVERVIEW OF THE CERRO AZUL FISHING DATA SET}

\subsection{INTRODUCTION}

The data we analyze is from 1980s while the historic site is more than 500 years prior. Dr. Joyce Marcus spent 1982-1986 excavating in Cerro Azul, Peru. Due to arid weather, architecture, fishing nets, and fish middens from 1100 to 1470 A.D were all well preserved. Dr. Marcus explored early "community self-sufficiency" and "community specialization" during Incan times. The Kingdom of Huarco contained two localities [1]. The coast proper was ruled by the Kingdom of Huarco, and the piedmont was ruled by Kingdom of Lunahuana. Both sites were later defeated by the Inca's in 1470. As in any society, the diet will typically differ based on a person social status. From bone remains found in different housing compounds, Marcus observed that different fish were eaten by different levels of society, such as the diets of the elites' versus that of the commoners'. While modern fishermen use equipment that allows them to catch a wider variety of species, the catches can be destined for local consumption or exported commercially to larger cities, such as Lima. As a result, the movements of certain catches that are targeted for commercial sale are more likely to be tracked than others, and fishermen may want to take more risks or more effort to find them. These factors will be key to the model developed earlier.

Wirth states that, "Each city, like every other object in nature, is, in a sense, unique" [17]. However, according to [17], "culture change in the direction of increased scale and complexity can occur in varied ways. I suggest that the cultural ecologists should do as others have and view this variety as a source of stimulation for theory-building." As Jayoussi presented in his PhD Thesis [19] a complex system can viewed as different levels of granularity relative to the questions that we wish to ask about it. Similarly, we also 
implement the several levels of analysis here; the entire period of the survey; monthly; weekly; and daily. In this research we investigate the potential for applying multi-objective cultural algorithms models of fishing patterns at the weekly and daily scales by using suitable-tools from Artificial Intelligence and Data Science to the existing archaeological data from a prehistoric fishing center, Cerro Azul. We begin by describing the basic structure of the Database system developed to support model development from the raw data in Section 2.1. Then, in Section 2.2 we briefly describe the Graphical User Interface to the Cerro Azul Database and illustrate how it can support queries at a variety of spatial and temporal scales. Section 2.3 provides our conclusions.

\subsection{AN OVERVIEW OF THE DATABASE CONTENT AS A COMPLEX SYSTEM}

Drs. Joyce Marcus and Maria Rostworowski led a team of researchers from The University of Michigan from 1982 through 1986 to excavate five seasons of research at ancient nearby site of Cerro Azul. Later, in the last three years of their project, they began recording the catch of every boat that returned to the Capitanian del Puerto with the cooperation of the local government. In addition, further data on fishing was collected from Peru's Instituto del Mar, [1]. Marcus refers to the fishermen as "Artisanal" Fishermen in the sense that they are small scale and independent entities that can provide for both local consumption and export. The dataset consists of 6013 records. Each record has the following properties:

1. Relates to exactly one fishing trip.

2. Contains fish from only one site location. (main source)

3. Contains fish belonging to only one species (main catch).

4. Fishermen always departed from the home site (Cerro Azul). 
The fishing activity around Cerro Azul is a complex system that has many different parts that interact with each other. We can view the different levels as Macro, Meso and Micro in terms of their temporal scale. The three basic phases of ENSO constitute the Macro scale. The Meso scale is represented by the monthly statistics. The micro level corresponds to the days of the week for a given week. These form the basic structure of the Cerro Azul database constructed here. The basic structure of the database can be visualized in Figure 2.1. There we see how the basic variables are related to each other in the database.

The basic organization of the database reflects the most-coarse grained temporal measurement used here which is based upon the ENSO phases discussed earlier and summarized below. ENSO, the El Nino Southern Oscillation, consists of three phases: El Nino, La Nino, and back to normal. El Nino is the first phase of change from the normal weather pattern. It is the warming phase of the ENSO. El Nino means "The Little child, referring to baby Jesus" [10], this term originally applied to the lighter warming affect that happens around Christmas time. The El Nino not only affects temperature but also rainfall. The next phase, La Nina, is the cooling phase, with increased upwelling. La Nina is Spanish for "Little Girl". Sometimes there will be more than one La Nina during the same ENSO. The final phase of the ENSO is the "back to normal". At this point the weather has returned to pre-El Nino conditions along with the food chains. Using ENSO's cycles, our dataset of fishing trips is divided into three different temporal phases at the Macro level: Phase I, Phase II, and Phase III.

Table 2.1 gives the Peru Fishing Database Dictionary listing the data variables and their possible values. It shows that there are 6013 Identifiers, one for each fishing trip. We used Catches and Sites name and abbreviation for each species and site in the data analysis 
and the model. The fishermen caught 48 different catch types by travelling to 29 different sites. The number of Individual Catch trips range from 1 to 6013 catches and the Total Weight of Fishing Expedition's catch range from 0.2 to 1000 Kilograms. Meanwhile, the number of days for each fishing trip varies between 1 to 7 days. The desirability of each catch based upon modern preferences is given below:

$$
\begin{aligned}
& 3=\text { Highly Desirable (targeted) } \\
& 2=\text { Desirable (okay), and } \\
& 1=\text { Fall Back (opportunistic) }
\end{aligned}
$$

"Highly desirable" catches are targeted by fishermen as the most commercially desired fish. "Desirable" fish are still commercial in nature, but will attract less of a return. The "Fall Back" classification corresponds to more ubiquitous but less commercial catches - ones that reflect the result of fishing rather than the catch that was produced. Risk-wise, it is better to come back with something rather than nothing from a subsistence point of view.

In addition, there are six classifications of catches that reflect generic differences in species:

$$
\begin{aligned}
& \text { 1=Cartilaginous, } \\
& \text { 2=Boney, } \\
& 3=\text { Crustacean, } \\
& \text { 4=Mammal, } \\
& \text { 5=Penguin, and } \\
& 6=\text { Turtle }
\end{aligned}
$$




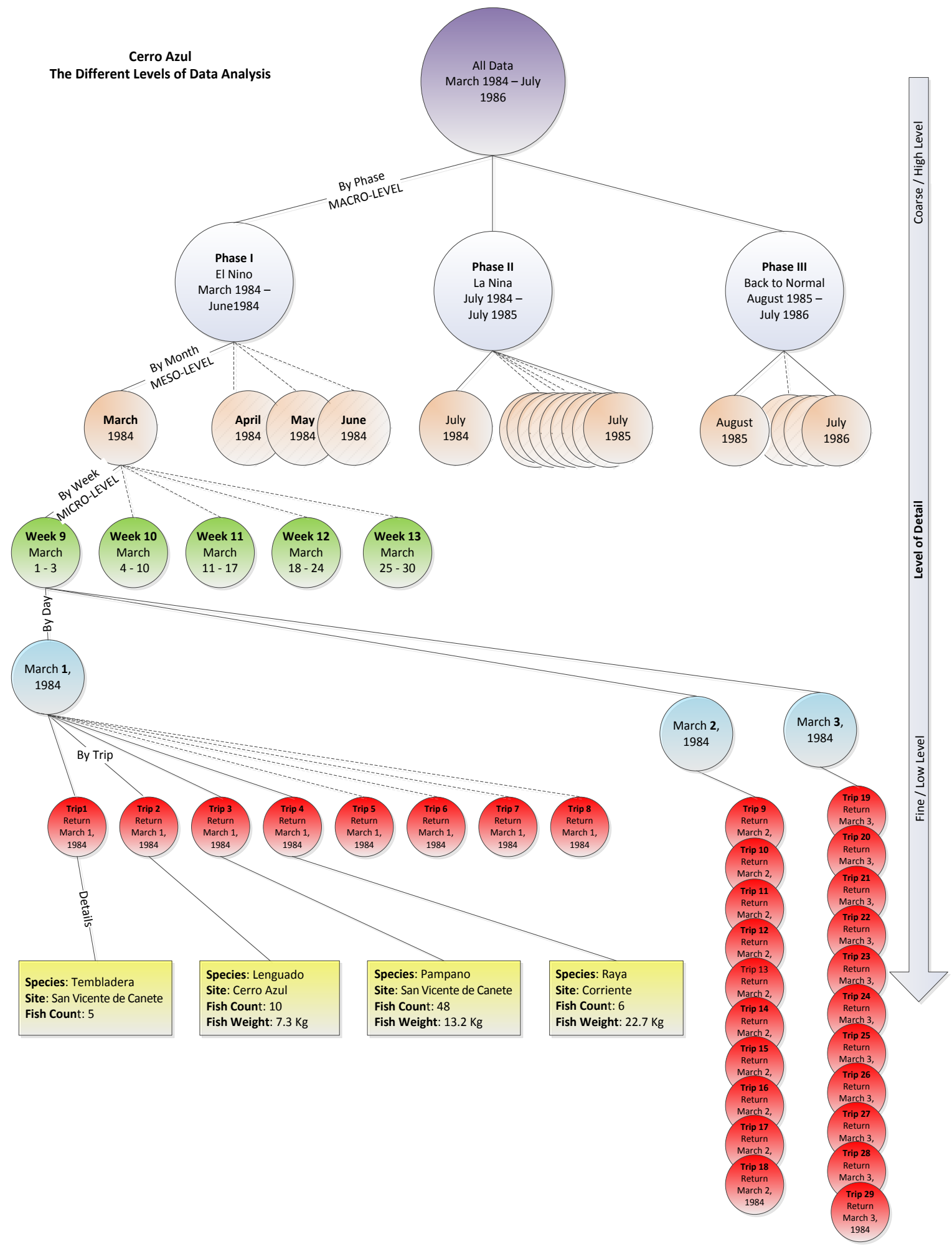

Figure 2.1: The different levels of data analysis: Macro, Meso and Micro. 
Table 2.1: Data Dictionary of the Peru Database

\begin{tabular}{|c|c|c|c|}
\hline Field Name & Description & Values & Comments \\
\hline ID & Unique Identifier & $(1-6013)$ & Fishing Trip ID \\
\hline Catch & Catch Name & $\begin{array}{l}48 \text { Different Catch } \\
\text { Types }\end{array}$ & \\
\hline Site & Site Name & 29 Different Sites & \\
\hline Count & Number of Individual Catch & $(1-6000)$ & \\
\hline Weight & $\begin{array}{l}\text { Total Weight of Fishing Expedition in } \\
\text { Kilograms }\end{array}$ & $(0.2-1000)$ & \\
\hline Depart Date & Departure Date of Fishing Expedition & $\begin{array}{l}(02 / 29 / 1984 \\
07 / 27 / 1986) \\
\end{array}$ & \\
\hline Return Date & Return Date of Fishing Expedition & $\begin{array}{l}(03 / 01 / 1984 \\
07 / 28 / 1986)\end{array}$ & \\
\hline $\begin{array}{ll}\text { Trip } & \text { Duration } \\
\text { (Length) } & \\
\end{array}$ & Number of Days for Fishing Expedition & $(1-7)$ & \\
\hline $\begin{array}{l}\text { Round Trip } \\
\text { Distance From CA }\end{array}$ & $\begin{array}{l}\text { Round Trip Distance from CA (Extended) in } \\
\text { Kilometers }\end{array}$ & $(0-198)$ & round trip dist. in $\mathrm{KMs}$ \\
\hline Phase & Phase of Return date Fishing Expedition & $(1-3)$ & $\begin{array}{l}1=\text { Residual El Nino, } 2=\mathrm{La} \text { Nina, } \\
3=\text { Back To Normal }\end{array}$ \\
\hline $\begin{array}{l}\text { Return Day of } \\
\text { Week }\end{array}$ & $\begin{array}{l}\text { Day of the Week for Fishing Expedition } \\
\text { Return }\end{array}$ & $(1-7)$ & $\begin{array}{l}1=\text { Monday, } 2=\text { Tuesday } \ldots, \\
6=\text { Friday, } 7=\text { Saturday }\end{array}$ \\
\hline $\begin{array}{l}\text { Num. of DOW } \\
\text { using RtnDate }\end{array}$ & $\begin{array}{l}\text { Number of Days of the Week (using Return } \\
\text { Date) that occurred in that month }\end{array}$ & $(4,5)$ & $\begin{array}{l}\text { Number of } \mathrm{M}, \mathrm{T}, \mathrm{W}, \mathrm{R}, \mathrm{F}, \mathrm{S}, \mathrm{S} \text { in } \\
\text { month }\end{array}$ \\
\hline $\begin{array}{l}\text { Site Loc North to } \\
\text { South }\end{array}$ & $\begin{array}{l}\text { Ordering all site locations from North to } \\
\text { South }\end{array}$ & & $\begin{array}{l}\text { numbers are used for sorting } \\
\text { purposes only and do not }\end{array}$ \\
\hline Site Relative to CA & $\begin{array}{l}\text { Site Location relative to CA (Extended) } \\
\text { Includes Faro and La Centinela }\end{array}$ & $(1-3)$ & $\begin{array}{l}1=\text { North of CA, 2=Cerro Azul, } \\
3=\text { South of CA }\end{array}$ \\
\hline Species Category & Classification of Catch & $(1-6)$ & $\begin{array}{l}1=\text { Cartilaginous }, 2=\text { Boney, } \\
3=\text { Crustacean, } 4=\text { Mammal, } 5=\text { Penguin, } \\
6=\text { Turtle }\end{array}$ \\
\hline Comments & changes or notes to data & $\begin{array}{l}\text { Count updated, } \\
\text { sites combined. }\end{array}$ & \\
\hline Depart Year & Fishing Expedition-Depart Year & $(1984-1986)$ & \\
\hline Depart Month & Fishing Expedition-Depart Month & $(1-12)$ & \\
\hline Depart Day & Fishing Expedition-Depart Day & $(1-31)$ & \\
\hline Return Year & Fishing Expedition - Return Year & $(1984-1986)$ & \\
\hline Return Month & Fishing Expedition - Return Month & $(1-12)$ & \\
\hline Return Day & Fishing Expedition - Return Day & $(1-31)$ & \\
\hline Desirability & $\begin{array}{l}\text { Desirability of species (decision by } \\
\text { Fishermen) }\end{array}$ & $(1-3)$ & $\begin{array}{l}3 \text { Highly Desirable, } \\
2=\text { Desirable, } \\
1=\text { Fall Back }\end{array}$ \\
\hline Indicator Fish & species that signify climate change & true or false & sensitive to temperature \\
\hline
\end{tabular}


Table 2.2 shows the catch list type based on categories of the Catches. The majority of catch types are boney fish, followed by cartilaginous. Table 2.3 provides a description of the three temporal phases of ENSO as used in our classification. They are based upon the temperature charts presented earlier. Phase I (El Nino) runs from March 1 to June 30, 1984. Phase II (La Nina) runs from July 1, 1984 to end of July 1985. Phase III (Back to Normal) runs from August 1, 1985 to end of July 1986.

We analyze the Catch list, type of catch, desirability of the catch for each of the three Phases as shown in Table 2.4. It shows the Catch list, type, their commercial desirability, and whether they were caught in each of the three Phases given above. Note that La Nina contains the diverse set of catches. The represent a combination of catches returning to the area as the water warms along with those catch types that are yet to leave for warmer waters.

Table 2.5 shows the Trip Count for each Catch Type by Phase and the percentage of the catch for each phase. The data shows that we have 714 trips for phase I, 4305 trips for phase II, and 994 trips for phase III. The number of trips for La Nina reflects the fact that it is the only one of the three that is completely charted along with the fact that there is a much greater variety of catch types as a result of the mixing of water temperatures. 
Table 2.2: Listing of Catch Type

\begin{tabular}{|l|l|l|l|}
\hline Cartilaginous & Boney & Crustacean & Mammal \\
\hline Azul & ayanque & Langostino & chancho marino \\
\hline guitarra & bonito & & \\
\hline pejegallo & caballa & & \\
\hline tembladera & cabinsa & & \\
\hline tollo & chita & & \\
\hline & cojinova & & \\
\hline & jurel & & \\
\hline & lenguado & & \\
\hline & liza & & \\
\hline & lorna & & \\
\hline & mismis & & \\
\hline & mojarilla & & \\
\hline & pampano & & \\
\hline & pejerrey & & \\
\hline & pintadilla & & \\
\hline & volador & & \\
\hline & zorro & & \\
\hline
\end{tabular}

Table 2.5 gives a list of all sites that were said to have been the primary visit location for each trip. For each site, their one way and round-trip distances are given in Kilometers. Their relative position, North and South of Cerro Azul, is given as well. Relative position is very important when it comes to the scheduling of fishing trips in response to phase, seasonal, and local weather changes. From the table it is clear that there were more northern sites targeted than southern ones. This might reflect the important commercial value of warm water fish to the local fishermen. Note that Cerro Azul, Faro, and La Centinel were assumed to be part of one general location, with a round trip distance of $1 \mathrm{~km}$ for each. 
Table 2.6 gives the percentage of trips for each catch in each period. The four most pervasive catches were Lorna, Mismis, Raya, and Tollo. They range from desirable on the one hand to fall back on the other. These catches persist through all three levels of ENSO and represent those catches that were sustainable throughout the ENSO period. Overall, almost four times as many trips were taken in La Nina than in the other two phases. Perhaps this reflects the diversity as well as the unpredictability of this transitional phase over time.

Table 2.3: Temporal Categories for Phases I. II and III

\begin{tabular}{|l|c|c|l|}
\hline Phase & Weather Pattern & Start Date & End Date \\
\hline Phase I & Residual El Niño & March 1, 1984 & June 30, 1984 \\
\hline Phase II & La Nina & July 1, 1984 & July 31, 1985 \\
\hline Phase III & Back to Normal & August 1, 1985 & July 31, 1986 \\
\hline
\end{tabular}


Table 2.4: The Catch list, type, and desirability for all three Phases

\begin{tabular}{|c|c|c|c|c|c|c|}
\hline & Name & Catch Type & Desirability & Phase I - & Phase II - & Phase III \\
\hline 1 & albacora & Boney & High / Targeted & & $\mathrm{Y}$ & \\
\hline 2 & anchoveta & Boney & Okay & & & $\mathrm{Y}$ \\
\hline 3 & angelona & Cartilaginous & Fall Back & $\mathrm{Y}$ & $\mathrm{Y}$ & $\mathrm{Y}$ \\
\hline 4 & ayanque & Boney & Okay & & $\mathrm{Y}$ & \\
\hline 5 & azul & Cartilaginous & Fall Back & $\mathrm{Y}$ & $\mathrm{Y}$ & $\mathrm{Y}$ \\
\hline 6 & bonito & Boney & High & $\mathrm{Y}$ & $\mathrm{Y}$ & $\mathrm{Y}$ \\
\hline 7 & burro & Boney & Okay & & $\mathrm{Y}$ & \\
\hline 8 & caballa & Boney & Okay & $\mathrm{Y}$ & $\mathrm{Y}$ & $\mathrm{Y}$ \\
\hline 9 & cabinsa & Boney & Okay & & $\mathrm{Y}$ & \\
\hline 10 & cabrilla & Boney & Okay & $\mathrm{Y}$ & $\mathrm{Y}$ & $\mathrm{Y}$ \\
\hline 11 & cachema & Boney & Fall Back & & $\mathrm{Y}$ & \\
\hline 12 & cangrejo & Crustacean & Fall Back & & $\mathrm{Y}$ & \\
\hline 13 & chancho & Mammal & Fall Back & $\mathrm{Y}$ & $\mathrm{Y}$ & $\mathrm{Y}$ \\
\hline 14 & chita & Boney & Okay & & $\mathrm{Y}$ & $\mathrm{Y}$ \\
\hline 15 & coco & Boney & Okay & $\mathrm{Y}$ & $\mathrm{Y}$ & \\
\hline 16 & cojinova & Boney & High / Targeted & $\mathrm{Y}$ & & $\mathrm{Y}$ \\
\hline 17 & corvina & Boney & High / Targeted & $\mathrm{Y}$ & $\mathrm{Y}$ & $\bar{Y}$ \\
\hline 18 & cristalino & Cartilaginous & Fall Back & $\mathrm{Y}$ & $\mathrm{Y}$ & $\mathrm{Y}$ \\
\hline 19 & diamante & Cartilaginous & Fall Back & $\mathrm{Y}$ & $\mathrm{Y}$ & \\
\hline 20 & guitarra & Cartilaginous & Fall Back & $\mathrm{Y}$ & $\mathrm{Y}$ & $\bar{Y}$ \\
\hline 21 & jurel & Boney & Okay & $\mathrm{Y}$ & $\mathrm{Y}$ & \\
\hline 22 & langostino & Crustacean & High / Targeted & $\mathrm{Y}$ & $\mathrm{Y}$ & \\
\hline 23 & lenguado & Boney & Okay & $\mathrm{Y}$ & $\mathrm{Y}$ & $\mathrm{Y}$ \\
\hline 24 & lisa & Boney & Okay & & $\mathrm{Y}$ & $\mathrm{Y}$ \\
\hline 25 & lorna & Boney & Okay & $\mathrm{Y}$ & $\mathrm{Y}$ & $\mathrm{Y}$ \\
\hline 26 & machete & Boney & Fall Back & & $\mathrm{Y}$ & \\
\hline 27 & mantaraya & Cartilaginous & Fall Back & $\mathrm{Y}$ & & \\
\hline 28 & mismis & Boney & Okay & $\mathrm{Y}$ & $\mathrm{Y}$ & $\mathrm{Y}$ \\
\hline 29 & mojarilla & Boney & Okay & $\mathrm{Y}$ & $\mathrm{Y}$ & \\
\hline 30 & pampano & Boney & High / Targeted & $\mathrm{Y}$ & & \\
\hline 31 & pejegallo & Cartilaginous & Fall Back & $\mathrm{Y}$ & $\mathrm{Y}$ & $\mathrm{Y}$ \\
\hline 32 & pejerrey & Boney & Okay & & & $\mathrm{Y}$ \\
\hline 33 & pez dama & Boney & Fall Back & $\mathrm{Y}$ & $\mathrm{Y}$ & \\
\hline 34 & pez gato & Boney & Fall Back & & $\mathrm{Y}$ & $\mathrm{Y}$ \\
\hline 35 & pez martillo & Cartilaginous & Fall Back & & $\mathrm{Y}$ & \\
\hline 36 & pez rata & Cartilaginous & Fall Back & $\mathrm{Y}$ & $\mathrm{Y}$ & $\mathrm{Y}$ \\
\hline 37 & pinguino & Bird & Fall Back & & $\mathrm{Y}$ & \\
\hline 38 & pintadilla & Boney & High / Targeted & $\mathrm{Y}$ & $\mathrm{Y}$ & $\mathrm{Y}$ \\
\hline 39 & raya & Cartilaginous & Fall Back & $\mathrm{Y}$ & $\mathrm{Y}$ & $\mathrm{Y}$ \\
\hline 40 & robalo & Boney & Okay & & $\mathrm{Y}$ & $\bar{Y}$ \\
\hline 41 & sardina & Boney & Okay & & $\mathrm{Y}$ & \\
\hline 42 & sierra & Boney & Okay & $\mathrm{Y}$ & & \\
\hline 43 & tembladera & Cartilaginous & Fall Back & $\mathrm{Y}$ & $\mathrm{Y}$ & $\mathrm{Y}$ \\
\hline 44 & tollo & Cartilaginous & Fall Back & $\mathrm{Y}$ & $\mathrm{Y}$ & $\mathrm{Y}$ \\
\hline 45 & tortuga & Reptile & Fall Back & & $\mathrm{Y}$ & \\
\hline 46 & trambollo & Boney & Okay & & $\mathrm{Y}$ & \\
\hline 47 & volador & Boney & Fall Back & & & $\mathrm{Y}$ \\
\hline 48 & zorro & Boney & Okay & $\mathrm{Y}$ & $\mathrm{Y}$ & $\mathrm{Y}$ \\
\hline
\end{tabular}


Table 2.5: The Site Distances from Cerro Azul.

\begin{tabular}{|l|l|l|l|}
\hline Site Name & $\begin{array}{l}\text { Oner Way } \\
\text { Distance (KM) }\end{array}$ & $\begin{array}{l}\text { Round Trip } \\
\text { Distance (KM) }\end{array}$ & Relative to CA \\
\hline San Bartolo & 78 & 156 & North \\
\hline San Pedro & 66 & 132 & North \\
\hline Cerro Blanco & 54 & 108 & North \\
\hline Mala & 44 & 88 & North \\
\hline Playa Asia & 33 & 66 & North \\
\hline Isla Asia & 31 & 62 & North \\
\hline Asia & 28 & 56 & North \\
\hline Mal Paso & 25 & 50 & North \\
\hline Playa Sarapampa & 23 & 46 & North \\
\hline Valdivia & 18 & 36 & North \\
\hline Los Leones & 16 & 32 & North \\
\hline Isla Corriente & 10 & 20 & North \\
\hline Corriente & 9 & 18 & North \\
\hline Gallardo & 7 & 14 & North \\
\hline Los Lobos & 6 & 12 & North \\
\hline Los Reyes & 3 & 6 & North \\
\hline Cerro Azul & 0.5 & 1 & Cerro Azul \\
\hline Faro & 0.5 & 1 & Cerro Azul \\
\hline La Centinel & 0.5 & 1 & Cerro Azul \\
\hline Puente Tabla & 2 & 4 & South \\
\hline Playa La Costa & 3 & 6 & South \\
\hline Santa Bárbara & 5 & 10 & South \\
\hline San Vicente de Canete & 9 & 18 & South \\
\hline Playa Hermosa & 10 & 20 & South \\
\hline Boca del Rio & 14 & 28 & South \\
\hline Herbay & 16 & 32 & South \\
\hline Cinch Cruces & 28 & 56 & South \\
\hline Colorado & 39 & 78 & South \\
\hline Jaway & 40 & 80 & South \\
\hline Tambo de Mora & 58 & 116 & South \\
\hline Monzo & 99 & 198 & South \\
\hline & & & \\
\hline
\end{tabular}


Table 2.6: The Trip Count for each Catch Type by Phase

\begin{tabular}{|c|c|c|c|c|c|c|c|c|c|}
\hline Des. & Catch Type & Phase I & $\mathrm{Ph} . \mathrm{I} \%$ & Phase II & Ph. II \% & Phase III & $\mathrm{PhIII} \mathrm{\%}$ & SUM & All P \% \\
\hline 1 & albacora & & & 1 & $0.0 \%$ & & & 1 & $0.0 \%$ \\
\hline 2 & anchoveta & 1 & $0.1 \%$ & & & 1 & $0.1 \%$ & 2 & $0.0 \%$ \\
\hline 3 & angelona & 1 & $0.1 \%$ & 7 & $0.2 \%$ & 1 & $0.1 \%$ & 9 & $0.1 \%$ \\
\hline 2 & ayanque & & & 22 & $0.5 \%$ & & & 22 & $0.4 \%$ \\
\hline 3 & azul & 38 & $5.3 \%$ & 80 & $1.9 \%$ & 116 & $11.7 \%$ & 234 & $3.9 \%$ \\
\hline 1 & bonito & 33 & $4.6 \%$ & 87 & $2.0 \%$ & 13 & $1.3 \%$ & 133 & $2.2 \%$ \\
\hline 2 & burro & & & 2 & $0.0 \%$ & & & 2 & $0.0 \%$ \\
\hline 2 & caballa & 6 & $0.8 \%$ & 12 & $0.3 \%$ & 4 & $0.4 \%$ & 22 & $0.4 \%$ \\
\hline 2 & cabinsa & & & 4 & $0.1 \%$ & & & 4 & $0.1 \%$ \\
\hline 2 & cabrilla & 1 & $0.1 \%$ & 3 & $0.1 \%$ & 1 & $0.1 \%$ & 5 & $0.1 \%$ \\
\hline 3 & cachema & & & 1 & $0.0 \%$ & & & 1 & $0.0 \%$ \\
\hline 3 & cangrejo & & & 1 & $0.0 \%$ & & & 1 & $0.0 \%$ \\
\hline 3 & chancho marino. & 54 & $7.6 \%$ & 294 & $6.8 \%$ & 150 & $15.1 \%$ & 498 & $8.3 \%$ \\
\hline 2 & chita & & & 45 & $1.0 \%$ & 11 & $1.1 \%$ & 56 & $0.9 \%$ \\
\hline 2 & coco & 9 & $1.3 \%$ & 1 & $0.0 \%$ & & & 10 & $0.2 \%$ \\
\hline 1 & cojinova & 1 & $0.1 \%$ & & & 45 & $4.5 \%$ & 46 & $0.8 \%$ \\
\hline 1 & corvina & 3 & $0.4 \%$ & 3 & $0.1 \%$ & 5 & $0.5 \%$ & 11 & $0.2 \%$ \\
\hline 3 & cristalino & 2 & $0.3 \%$ & 39 & $0.9 \%$ & 3 & $0.3 \%$ & 44 & $0.7 \%$ \\
\hline 3 & diamante & 2 & $0.3 \%$ & 15 & $0.3 \%$ & & & 17 & $0.3 \%$ \\
\hline 3 & guitarra & 47 & $6.6 \%$ & 14 & $0.3 \%$ & 36 & $3.6 \%$ & 97 & $1.6 \%$ \\
\hline 2 & jurel & 18 & $2.5 \%$ & 4 & $0.1 \%$ & & & 22 & $0.4 \%$ \\
\hline 1 & langostino & 73 & $10.2 \%$ & 4 & $0.1 \%$ & & & 77 & $1.3 \%$ \\
\hline 2 & lenguado & 38 & $5.3 \%$ & 28 & $0.7 \%$ & 1 & $0.1 \%$ & 67 & $1.1 \%$ \\
\hline 2 & lisa & & & 2 & $0.0 \%$ & 13 & $1.3 \%$ & 15 & $0.2 \%$ \\
\hline 2 & lorna & 46 & $6.4 \%$ & 1173 & $27.2 \%$ & 72 & $7.2 \%$ & 1291 & $21.5 \%$ \\
\hline 3 & machete & & & 3 & $0.1 \%$ & & & 3 & $0.0 \%$ \\
\hline 3 & mantaraya & 1 & $0.1 \%$ & & & & & 1 & $0.0 \%$ \\
\hline 2 & mismis & 11 & $1.5 \%$ & 895 & $20.8 \%$ & 17 & $1.7 \%$ & 923 & $15.4 \%$ \\
\hline 2 & mojarilla & 1 & $0.1 \%$ & 6 & $0.1 \%$ & & & 7 & $0.1 \%$ \\
\hline 1 & pampano & 12 & $1.7 \%$ & & & & & 12 & $0.2 \%$ \\
\hline 3 & pejegallo & 23 & $3.2 \%$ & 159 & $3.7 \%$ & 35 & $3.5 \%$ & 217 & $3.6 \%$ \\
\hline 3 & pejerrey & & & & & 98 & $9.9 \%$ & 98 & $1.6 \%$ \\
\hline 3 & pez dama & 1 & $0.1 \%$ & 3 & $0.1 \%$ & & & 4 & $0.1 \%$ \\
\hline 3 & pez gato & & & 4 & $0.1 \%$ & 2 & $0.2 \%$ & 6 & $0.1 \%$ \\
\hline 3 & pez martillo & & & 13 & $0.3 \%$ & & & 13 & $0.2 \%$ \\
\hline 3 & pez rata & 5 & $0.7 \%$ & 32 & $0.7 \%$ & 5 & $0.5 \%$ & 42 & $0.7 \%$ \\
\hline 3 & pinguino & & & 1 & $0.0 \%$ & & & 1 & $0.0 \%$ \\
\hline 2 & pintadilla & 7 & $1.0 \%$ & 12 & $0.3 \%$ & 5 & $0.5 \%$ & 24 & $0.4 \%$ \\
\hline 3 & raya & 128 & $17.9 \%$ & 608 & $14.1 \%$ & 168 & $16.9 \%$ & 904 & $15.0 \%$ \\
\hline 1 & robalo & & & 1 & $0.0 \%$ & 1 & $0.1 \%$ & 2 & $0.0 \%$ \\
\hline 2 & sardina & & & 4 & $0.1 \%$ & & & 4 & $0.1 \%$ \\
\hline 2 & sierra & 6 & $0.8 \%$ & & & & & 6 & $0.1 \%$ \\
\hline 3 & tembladera & 5 & $0.7 \%$ & 6 & $0.1 \%$ & 15 & $1.5 \%$ & 26 & $0.4 \%$ \\
\hline 2 & tollo & 136 & $19.0 \%$ & 653 & $15.2 \%$ & 138 & $13.9 \%$ & 927 & $15.4 \%$ \\
\hline 3 & tortuga & & & 2 & $0.0 \%$ & & & 2 & $0.0 \%$ \\
\hline 2 & trambollo & & & 1 & $0.0 \%$ & & & 1 & $0.0 \%$ \\
\hline 3 & volador & & & 7 & $0.2 \%$ & 2 & $0.2 \%$ & 9 & $0.1 \%$ \\
\hline \multirow[t]{2}{*}{2} & zorro & 5 & $0.7 \%$ & 53 & $1.2 \%$ & 36 & $3.6 \%$ & 94 & $1.6 \%$ \\
\hline & SUM & 714 & Phase I & 4305 & Phase II & 994 & Phase & 6013 & Total \\
\hline
\end{tabular}


In Table 2.7, we list the Trip Count for each Catch Type for all Phases, sorted by Trip Count from largest to smallest in each phase. Observe that the higher desirable fish are caught more frequently in the El Nino and La Nina phases, whereas the top catches in the Back to Normal phase are mostly okay or fall back. In addition, one can see certain species change their frequencies over time. For example, Pejegallo was the focus for 23 trips in the residual El Nino phase, 159 trips in the La Nina phase, and 35 trips in the Back to Normal phase. Another catch type, Pejerrey, went from 0 in El Nino, to 3 in La Nina, and 98 in the Back to Normal phases. Figure 2.2 shows how the location of even a single catch changes over time. As the week go on, the search for Mismis moves from colder waters in the south to Cerro Azul as the waters there get colder and become more attractive to Mismis.

Table 2.8 presents the Trip Count and corresponding percentage of the total trips for each site by phase. In that Table we see that while Cerro Azul and Faro dominate in terms of site location over all three phases as might be expected, visits to the North and South differed markedly between the phases. There are more visits north to places like Asia in La Nina as warm water fish began to move back north to warmer waters. Trips to the south for colder water fish increased from El Nino to Back to Normal is indicated by increased visits to the Rio Canete during the period of observation. These changes probably reflect the attempts to track targeted fish as they moved into and out the region as a result of ENSO. 
Table 2.7: Trip Count based for Catch Type, Sorted by Trip Count by largest to smallest

\begin{tabular}{|c|c|c|c|c|c|c|c|c|}
\hline Des. & Catch & Phase I & Des. & Catch & Phase II & Des. & Catch & Phase III \\
\hline 2 & Tollo & 136 & 2 & lorna & 1173 & 3 & raya & 168 \\
\hline 1 & Raya & 128 & 2 & mismis & 895 & 3 & chancho & 150 \\
\hline 3 & langostino & 73 & 2 & tollo & 653 & 2 & tollo & 138 \\
\hline 1 & chancho & 54 & 3 & raya & 608 & 3 & azul & 116 \\
\hline 1 & Guitarra & 47 & 3 & chancho & 294 & 3 & pejerrey & 98 \\
\hline 2 & Lorna & 46 & 3 & pejegallo & 159 & 2 & lorna & 72 \\
\hline 1 & Azul & 38 & 1 & bonito & 87 & 1 & cojinova & 45 \\
\hline 2 & lenguado & 38 & 3 & azul & 80 & 3 & guitarra & 36 \\
\hline 3 & Bonito & 33 & 2 & zorro & 53 & 2 & zorro & 36 \\
\hline 1 & Pejegallo & 23 & 2 & chita & 45 & 3 & pejegallo & 35 \\
\hline 2 & Jurel & 18 & 3 & cristalino & 39 & 2 & mismis & 17 \\
\hline 3 & pampano & 12 & 3 & pez rata & 32 & 3 & tembladera & 15 \\
\hline 2 & Mismis & 11 & 2 & lenguado & 28 & 1 & bonito & 13 \\
\hline 2 & Coco & 9 & 2 & ayanque & 22 & 2 & lisa & 13 \\
\hline 2 & pintadilla & 7 & 3 & diamante & 15 & 2 & chita & 11 \\
\hline 2 & Caballa & 6 & 3 & guitarra & 14 & 1 & corvina & 5 \\
\hline 2 & Sierra & 6 & 3 & pez martillo & 13 & 3 & pez rata & 5 \\
\hline 1 & pez rata & 5 & 2 & caballa & 12 & 2 & pintadilla & 5 \\
\hline 1 & tembladera & 5 & 2 & pintadilla & 12 & 2 & caballa & 4 \\
\hline 2 & Zorro & 5 & 3 & angelona & 7 & 3 & cristalino & 3 \\
\hline 3 & Corvina & 3 & 3 & volador & 7 & 3 & pez gato & 2 \\
\hline 1 & Cristalino & 2 & 2 & mojarilla & 6 & 3 & volador & 2 \\
\hline 1 & diamante & 2 & 3 & tembladera & 6 & 2 & anchoveta & 1 \\
\hline 2 & anchoveta & 1 & 2 & cabinsa & 4 & 3 & angelona & 1 \\
\hline 1 & angelona & 1 & 2 & jurel & 4 & 2 & cabrilla & 1 \\
\hline 2 & Cabrilla & 1 & 1 & langostino & 4 & 2 & lenguado & 1 \\
\hline 3 & Cojinova & 1 & 3 & pez gato & 4 & 1 & robalo & 1 \\
\hline 1 & mantaraya & 1 & 2 & sardina & 4 & 1 & albacora & \\
\hline 2 & Mojarilla & 1 & 2 & cabrilla & 3 & 2 & ayanque & \\
\hline 1 & pez dama & 1 & 1 & corvina & 3 & 2 & burro & \\
\hline 3 & Albacore & & 3 & machete & 3 & 2 & cabinsa & \\
\hline 2 & Ayanque & & 3 & pez dama & 3 & 3 & cachema & \\
\hline 2 & Burro & & 2 & burro & 2 & 3 & cangrejo & \\
\hline 2 & Cabinsa & & 2 & lisa & 2 & 2 & coco & \\
\hline 1 & Cachema & & 3 & tortuga & 2 & 3 & diamante & \\
\hline 1 & Cangrejo & & 1 & albacora & 1 & 2 & jurel & \\
\hline 2 & Chita & & 3 & cachema & 1 & 1 & langostino & \\
\hline 2 & Lisa & & 3 & cangrejo & 1 & 3 & machete & \\
\hline 1 & Machete & & 2 & coco & 1 & 3 & mantaraya & \\
\hline 1 & Pejerrey & & 3 & pinguino & 1 & 2 & mojarilla & \\
\hline 1 & pez gato & & 1 & robalo & 1 & 1 & pampano & \\
\hline 1 & pez martillo & & 2 & trambollo & 1 & 3 & pez dama & \\
\hline 1 & Pinguino & & 2 & anchoveta & & 3 & pez martillo & \\
\hline 3 & Robalo & & 1 & cojinova & & 3 & pinguino & \\
\hline 2 & Sardina & & 3 & mantaraya & & 2 & sardina & \\
\hline 1 & Tortuga & & 1 & pampano & & 2 & sierra & \\
\hline 2 & Trambollo & & 3 & pejerrey & & 3 & tortuga & \\
\hline 1 & Volador & & 2 & sierra & & 2 & trambollo & \\
\hline \multicolumn{2}{|c|}{ Sum of Catch Count } & 714 & & & 4305 & & & 994 \\
\hline
\end{tabular}


Filters: YEAR $=1984$ and FISH = mismis and LOCATION $=$ All Locations and TRIP LENGTH $=$ All Trip Lengths

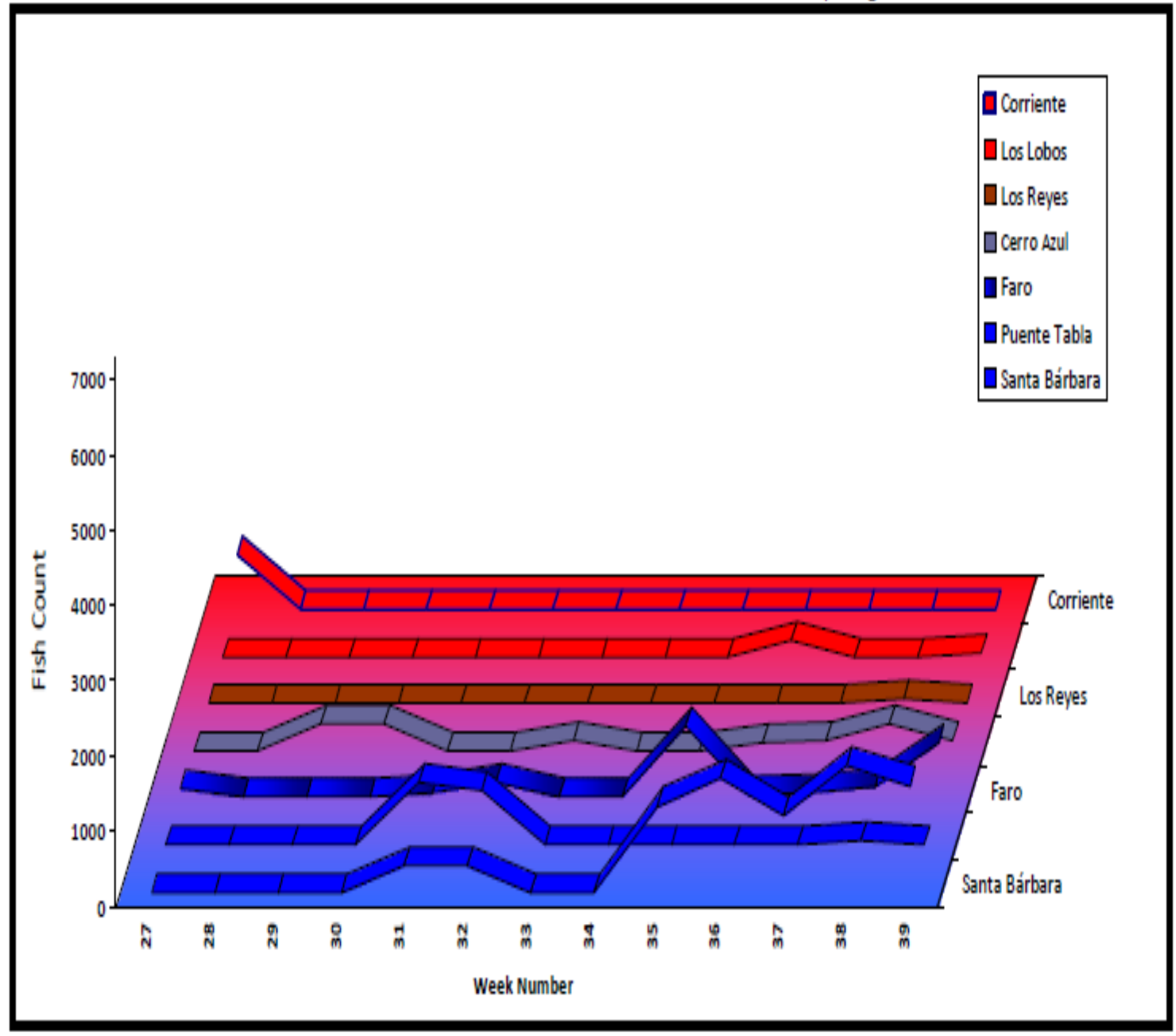

Figure 2.2: Catch Count by week for Mismis 
Table 2.8: The Trip Count and corresponding percentage for each Site by Phase

\begin{tabular}{|c|c|c|c|c|c|c|}
\hline Site & $\begin{array}{c}\text { Phase } \\
\text { I }\end{array}$ & $\begin{array}{c}\text { Phase I } \\
\%\end{array}$ & $\begin{array}{c}\text { Phase } \\
\text { II }\end{array}$ & $\begin{array}{c}\text { Phase } \\
\text { II \% }\end{array}$ & $\begin{array}{c}\text { Phase } \\
\text { III }\end{array}$ & $\begin{array}{l}\text { Phase } \\
\text { III \% }\end{array}$ \\
\hline Asia & 71 & $9.9 \%$ & 221 & $5.1 \%$ & 69 & $6.9 \%$ \\
\hline Boca del Rio & 5 & $0.7 \%$ & 17 & $0.4 \%$ & 24 & $2.4 \%$ \\
\hline Cerro Azul & 389 & $54.5 \%$ & 1553 & $36.1 \%$ & 526 & $52.9 \%$ \\
\hline Cerro Blanco & & $0.0 \%$ & 2 & $0.0 \%$ & & $0.0 \%$ \\
\hline Cinco Cruces & & $0.0 \%$ & & $0.0 \%$ & 1 & $0.1 \%$ \\
\hline Colorado & & $0.0 \%$ & 62 & $1.4 \%$ & 4 & $0.4 \%$ \\
\hline Corriente & 35 & $4.9 \%$ & 66 & $1.5 \%$ & 6 & $0.6 \%$ \\
\hline Faro & 96 & $13.4 \%$ & 1300 & $30.2 \%$ & 139 & $14.0 \%$ \\
\hline Gallardo & & $0.0 \%$ & & $0.0 \%$ & 4 & $0.4 \%$ \\
\hline Herbay & & $0.0 \%$ & & $0.0 \%$ & 8 & $0.8 \%$ \\
\hline Isla Asia & 3 & $0.4 \%$ & 1 & $0.0 \%$ & & $0.0 \%$ \\
\hline Isla Corriente & 1 & $0.1 \%$ & 103 & $2.4 \%$ & 10 & $1.0 \%$ \\
\hline Jaway & & $0.0 \%$ & 6 & $0.1 \%$ & 2 & $0.2 \%$ \\
\hline Los Leones & 8 & $1.1 \%$ & 6 & $0.1 \%$ & 3 & $0.3 \%$ \\
\hline Los Lobos & 7 & $1.0 \%$ & 28 & $0.7 \%$ & 10 & $1.0 \%$ \\
\hline Los Reyes & 4 & $0.6 \%$ & 188 & $4.4 \%$ & 6 & $0.6 \%$ \\
\hline Mal Paso & 7 & $1.0 \%$ & 13 & $0.3 \%$ & & $0.0 \%$ \\
\hline Mala & 1 & $0.1 \%$ & & $0.0 \%$ & & $0.0 \%$ \\
\hline Monzo & & $0.0 \%$ & 1 & $0.0 \%$ & & $0.0 \%$ \\
\hline Playa Hermosa & & $0.0 \%$ & 3 & $0.1 \%$ & & $0.0 \%$ \\
\hline Playa La Costa & 3 & $0.4 \%$ & 4 & $0.1 \%$ & & $0.0 \%$ \\
\hline Playa Sarapampa & 3 & $0.4 \%$ & & $0.0 \%$ & 1 & $0.1 \%$ \\
\hline Puente Tabla & & $0.0 \%$ & 30 & $0.7 \%$ & & $0.0 \%$ \\
\hline San Bartolo & & $0.0 \%$ & 2 & $0.0 \%$ & & $0.0 \%$ \\
\hline San Pedro & 2 & $0.3 \%$ & 2 & $0.0 \%$ & & $0.0 \%$ \\
\hline San Vicente de Canete & 35 & $4.9 \%$ & 441 & $10.2 \%$ & 156 & $15.7 \%$ \\
\hline Santa Bárbara & 43 & $6.0 \%$ & 255 & $5.9 \%$ & 25 & $2.5 \%$ \\
\hline Tambo de Mora & 1 & $0.1 \%$ & & $0.0 \%$ & & $0.0 \%$ \\
\hline Valdivia & & $0.0 \%$ & 1 & $0.0 \%$ & & $0.0 \%$ \\
\hline Sum & 714 & & 4305 & & 994 & \\
\hline
\end{tabular}




\subsection{THE STRUCTURE OF THE DATABASE INTERFACE}

This section will focus on the database interface and how it supports the data mining of spatial and temporal patterns of fishing behavior by both site location and catch that will be used to develop and test our model of agent-based fishing behavior here. The key index variables used to retrieve and display data used here are Fish ID and Catch ID. These will be used to express our hypotheses at the different levels of temporal and spatial granularity required to build the model. We discuss the data mining at the sites level turning our attention to the different sites and demonstrate the ability of the system to generate the statistical output by fish species for both fish count (catch count) and fish weight for the three different levels of temporal granularity.

The following, Figure 2.3 shows the Menu Screen developed by Dr. Reynolds and Khalid Kattan. The catch log screen takes a particular catch index (from 1 to 6013), and uses the location and fish maintenance tabs (tables) to allow extract the associated variables for that data record. The four other tabs correspond to customized data displays to be used to address queries related to key hypotheses. Examples of their use will be given later in this section.

Each of the two Maintenance tabs (catch and location) is associated with a specific indicator or index variable table as shown in Figure 2.4. A combination of those indicator values can be used to extract combinations of the descriptor variables pointed to by each. Figure 2.5 gives the Maintenance screen for Catches (Fish). The user can select a subset of catches by clicking on the appropriate boxes. Figure 2.6 gives the Maintenance screen for Location selection. Figure 2.6 provides the screen for Ad Hoc reporting that allows the joint 
selection of catch, location, phase etc. to shape the query results. The result of one such query is given as 3D Line Graph in Figure 2.8

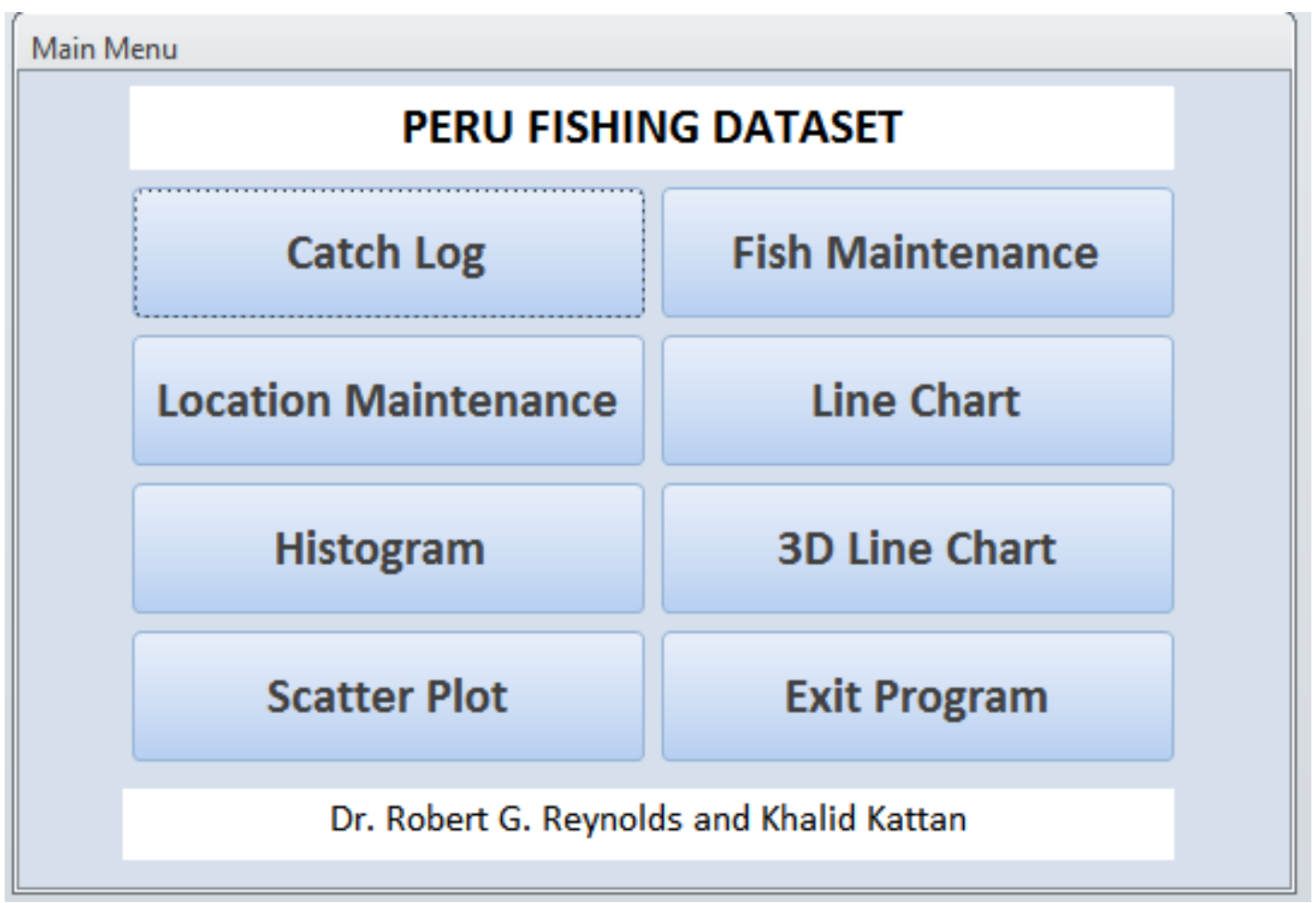

Figure 2.3: Menu Screen developed by Dr. Reynolds and Khalid Kattan 


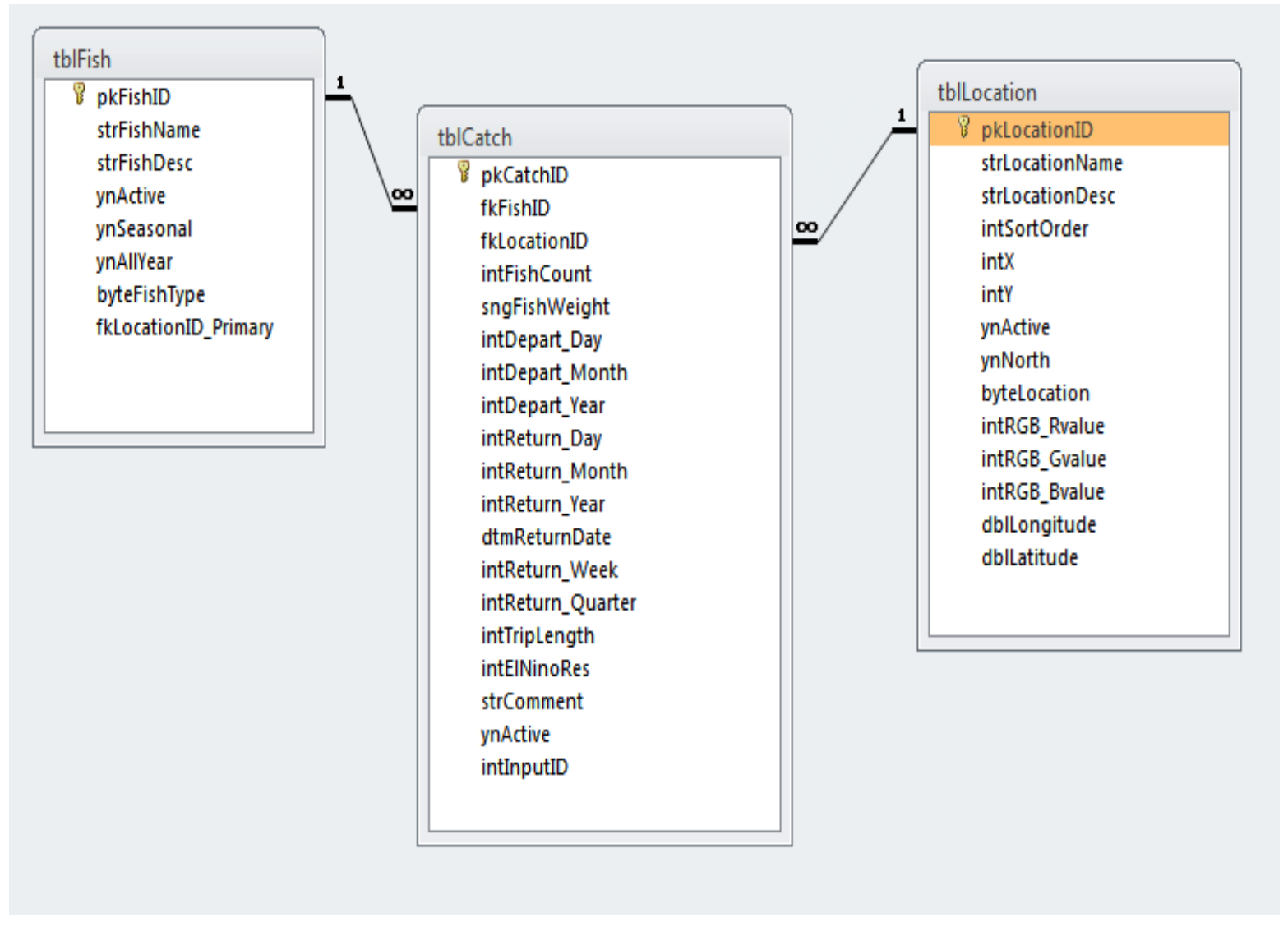

Figure 2.4: Displays the Database diagram with two index variables, fish and location ID

Notice the one-to-many table relationships between tblFish and tblCatch and between tblLocation and tblCatch. This is because for each recorded trip (in tblCatch), we only record the one Fish/Catch and the one Location it was caught at. 


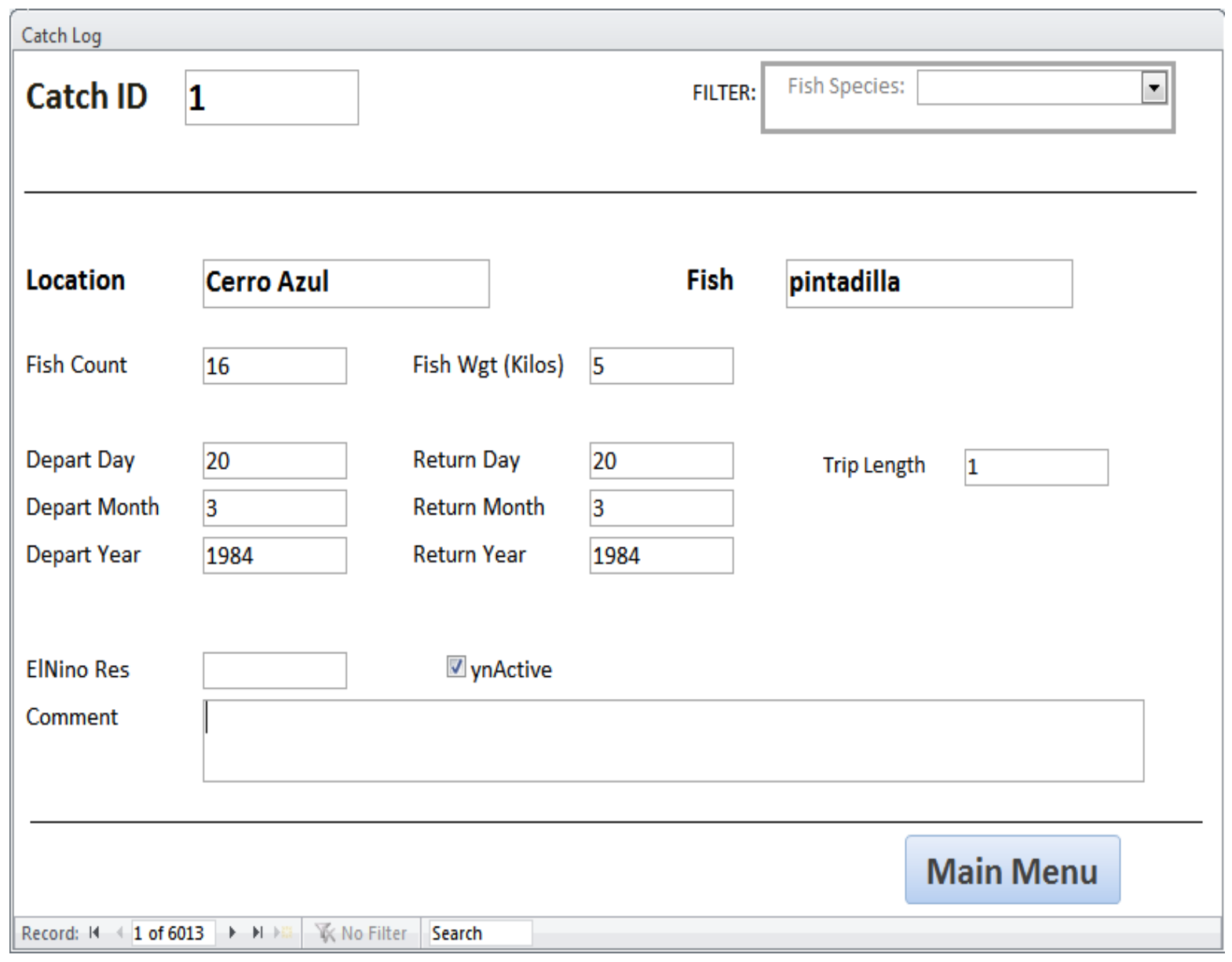

Figure 2.5: Gives the Catch Log screen result for catch ID 1. 


\begin{tabular}{|c|c|c|c|c|c|}
\hline \multicolumn{6}{|l|}{ Fish Maintenance } \\
\hline Fish Name & \multicolumn{2}{|c|}{ Fish Type } & \multirow{2}{*}{$\begin{array}{c}\text { Active } \\
\square\end{array}$} & \multirow[t]{2}{*}{ Fish Description } & \multirow[t]{2}{*}{$\Delta$} \\
\hline albacora & Cartilgenous & Boney & & & \\
\hline anchoveta & Cartilgenous & Boney & $\square$ & & \\
\hline angelona & Cartilgenous & Boney & $\square$ & & $\equiv$ \\
\hline ayanque & Cartilgenous & (0) Boney & 目 & ayanque & \\
\hline azul & (-) Cartilgenous & Boney & 目 & azul & \\
\hline bonito & Cartilgenous & ( ) Boney & 目 & bonito & \\
\hline burro & Cartilgenous & Boney & $\square$ & & \\
\hline caballa & Cartilgenous & ( ) Boney & $\nabla$ & caballa & \\
\hline cabinsa & Cartilgenous & ( ) Boney & $\nabla$ & cabinsa & \\
\hline cabrilla & Cartilgenous & Boney & $\square$ & & \\
\hline cachema & $\bigcirc$ Cartilgenous & Boney & $\square$ & & \\
\hline cacho & Cartilgenous & Boney & $\square$ & & \\
\hline camarón & Cartilgenous & Boney & $\square$ & & \\
\hline cangrejo & Cartilgenous & Boney & $\square$ & & \\
\hline chancho marino & Cartilgenous & Boney & 目 & chancho marino & \\
\hline chita & Cartilgenous & Boney & $\nabla$ & chita & \\
\hline$\Gamma$ & 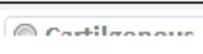 & An $\mathrm{n} n \mathrm{nn}$ & 回 & $\Gamma$ & \\
\hline & & & Main & Menu & \\
\hline
\end{tabular}

Figure 2.6: Gives the Fish Maintenance Screen.

Figure 2.6 shows the Fish Maintenance screen that allows the user to select a subset of variables for display. By setting Active field to yes, the fish species will be used in the result set. Setting the field to Active - no, allows the omission of a fish species. Similarly, in Figure 2.7, those sites selected as active will appear in the result set. 


\begin{tabular}{|c|c|c|c|c|c|c|c|c|c|c|c|}
\hline \multicolumn{12}{|l|}{ Location Maintenance } \\
\hline Location Name & \multicolumn{2}{|l|}{ Location Type } & \multirow{2}{*}{$\begin{array}{c}\text { Active } \\
\square\end{array}$} & \multicolumn{3}{|l|}{ Description } & \multicolumn{2}{|c|}{ Sort Order } & Longitude & Latitude & \multirow[t]{2}{*}{$\Delta$} \\
\hline Asia & (0) North O Cerro Azul & Osouth & & & 255 & 0 & 0 & 200 & -12.7873754 & .76 .5455461 & \\
\hline Boca del Rio & O North O Cerro Azul & (2) South & จ & & 60 & 170 & 215 & 2200 & & & \\
\hline Cerro Azul & O North @ Cerro Azul & OSouth & च & & 125 & 125 & 125 & 1400 & & & \\
\hline Cerro Blanco & O North O Cerro Azul & O South & 0 & & & & & & & & \\
\hline Cinco Cruces & O North O Cerro Azul & OSouth & 回 & & & & & & & & \\
\hline Colorado & O North O Cerro Azul & (2) South & $\nabla$ & & 0 & 0 & 255 & 2300 & & & \\
\hline Corriente & (0) North O Cerro Azul & OSouth & च & & 215 & 20 & 20 & 1000 & & & \\
\hline Faro & O North O Cerro Azul & (2) South & 甲 & & 115 & 135 & 135 & 1500 & & & \\
\hline Gallardo & (0) North O Cerro Azul & OSouth & च & & 200 & 30 & 30 & 1050 & & & \\
\hline Herbay & O North O Cerro Azul & (2) South & च & & 10 & 10 & 235 & 2250 & & & \\
\hline Isla Asia & (0 North O Cerro Azul & OSouth & 四 & & . & & & 400 & & & \\
\hline Isla Corriente & (0) North O Cerro Azul & OSouth & $\nabla$ & & 235 & 10 & 10 & 900 & & & \\
\hline & & & & Main Men & & & & & & & $=$ \\
\hline
\end{tabular}

Figure 2.7: This screen shows the Location Maintenance screen. 


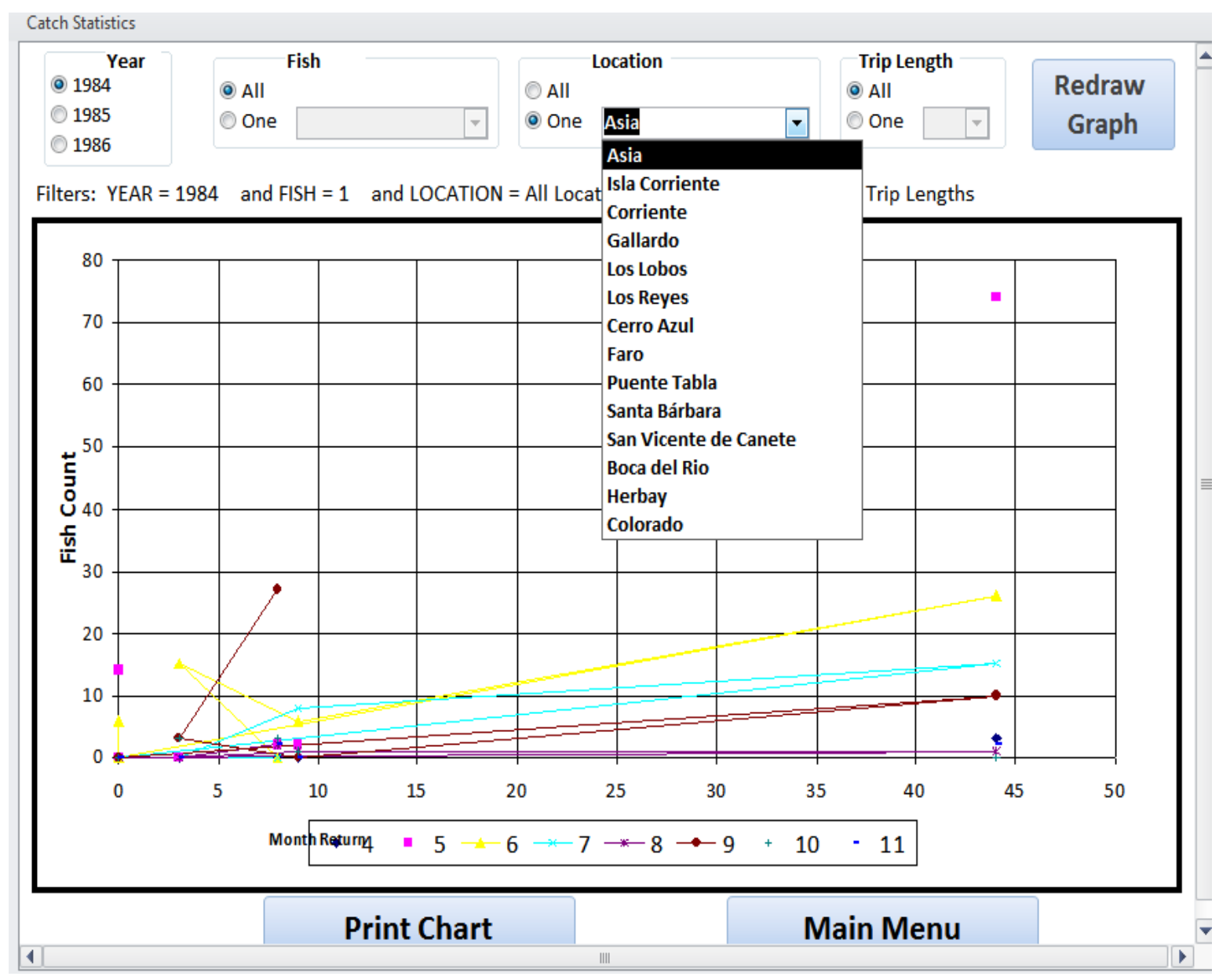

Figure 2.8: This screen shows the Ad-hoc Reporting.

Figure 2.8 shows the Ad-hoc Reporting screen. This screen allows interactive reporting by allowing different parameters to be selected (year, fish, location, trip length) to filter the output of a query. The report refreshes based on the user's parameters. 


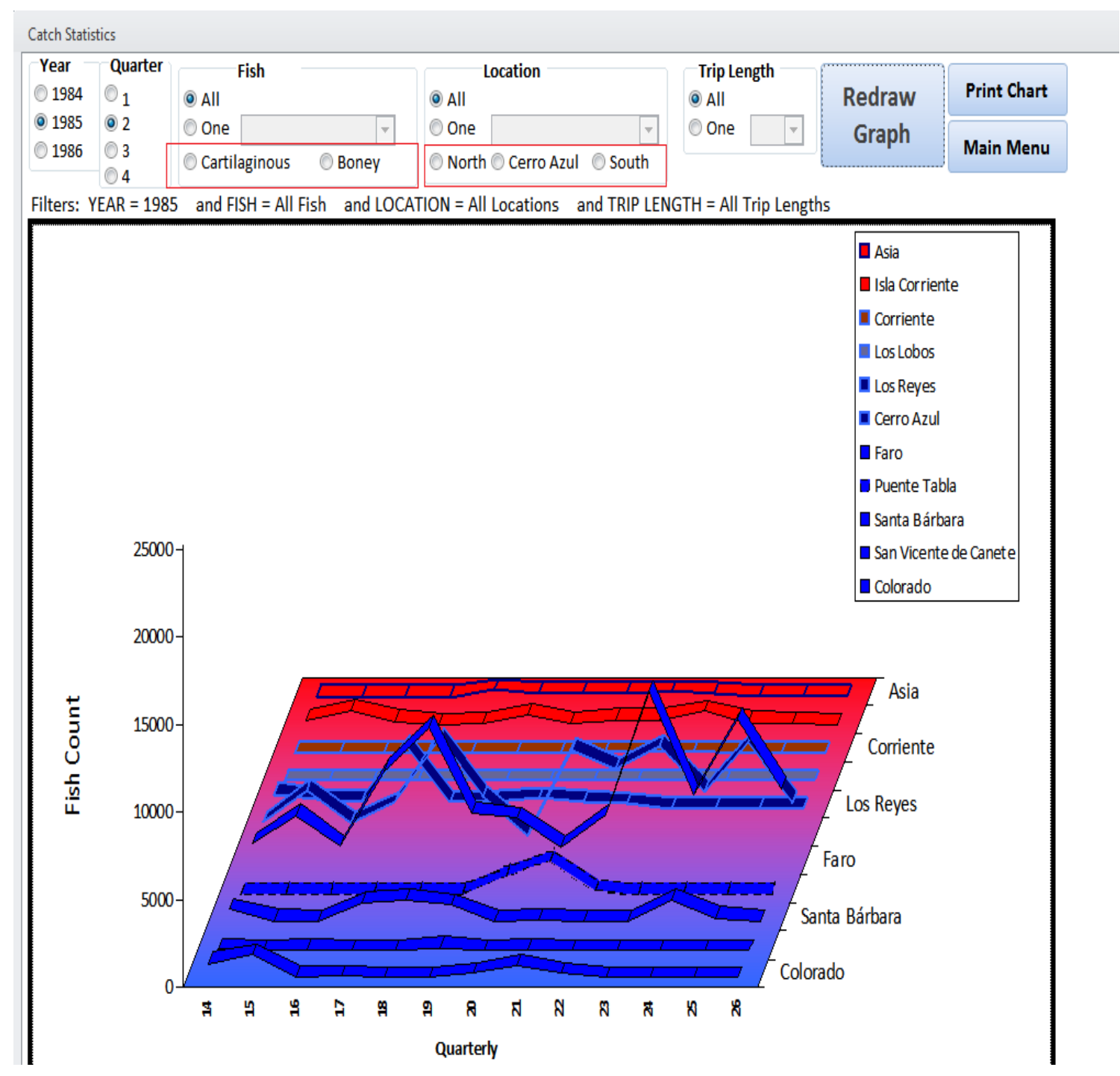

Figure 2.9: The Reporting by Species Type and Location Type.

In Figure 2.9, notice the background changes colors from red to blue as going from north (Asia) to south (Colorado). We chose red to represent the warmer sea temperature and blue to represent cooler.

\subsection{CONCLUSIONS}

In this Chapter we have described the basic temporal and spatial structure of the Cerro Azul knowledge base that was generated from Dr. Marcus's raw data. The Database 
Interface that facilitates the query and display of selected data records was also provided. This knowledge base will be the foundation upon which the Agent-Based fishing model will be produced in later chapters. While some basic trends were briefly mentioned here, in the next chapter we will present some data patterns at a variety of temporal and spatial scales that will provide the environmental assumptions around which the model will be created. 


\section{CHAPTER 3: DATA MINING AT THE MESO AND MICRO LEVELS}

\subsection{INTRODUCTION}

The original dataset contains 6013 records capturing fishing expeditions between early 1984 through mid-1986. These records capture the response of local fisherman to the warming and then cooling of the ocean. They are part of a complex system that can be assessed at a number of levels of granularity. In the previous chapter the database system that we developed for the application was introduced. We then used the system to determine whether the three basic phases of the ENSO event can be viewed as eliciting different responses from the fishermen at the macro level.

The results suggested that while there were some similarities between the three phases at the macro-level, the responses exhibited particular differences in the decision-space between them [20]. For example, certain catches such as Lorna and Mismis were caught in all three phases. On the other hand, the three phases differ in terms of the quality of their catches, and the effort taken to acquire them. This implies that our model needs to address these two conflicting goals. First, fishermen are after quality in terms of returns on their investment. Second, the fishermen want to make a sufficient investment in the fishing trip in order to produce a successful result. These two goals are conflicting in the sense that an expenditure of resources does not necessarily guarantee a quality catch. This can be viewed as a bi-objective problem, with two complementary goals.

In this chapter we examine each of the three phases at the meso- and micro-levels

relative to these two goals. The granularity of the meso-level is expressed here in terms of months. The micro-level corresponds to the days of the week within each of the months. Ultimately we will want our model to correspond to the daily decisions made by fishermen in 
each phase. In the next section we begin by distinguishing between the phases in terms of the quality of fish caught in each phase at the meso- and micro- scales. In section 3.3 we investigate resource expenditure invested in fishing trip based upon the catch type. We view distance travelled and the number of trips made as surrogates for the investment of effort in a given catch type here.

\subsection{THE-LEVEL: MONTHLY AND DAILY CHANGES IN QUALITY}

We begin by describing the catches in detail. Table 3.1 gives the name of each catch, along with its desirability from an economic standpoint, along with the Phases in which it was caught. Each Phase is defined over a corresponding set of dates and labeled with an abbreviation; REL is Residual El Nino; LN is La Nina; and BTN is Back to Normal. In the following table we show all 48 types of catches based on the desirability. Of those 48 , only 7 of these are rated as highly desirable which represent $14.58 \%, 20$ are rated as desirable are desirable which represents $41.67 \%$, finally, 21 are fall back which represents $43.75 \%$, highly desirable catches are ones. Our assumption is the highly desirable catches are more likely to be targeted by fishermen. Desirable ones may have commercial value also well. Fall Back catches are those that are not necessarily targeted for a trip but in order for the trip to be called successful something needs to be returned, even though they may have neglible commercial value. The commercial viability was determined by looking at comments by fishermen and published material. 
Table 3.1: Species catch during each phase for desirability

\begin{tabular}{|c|c|c|c|c|c|c|c|}
\hline & & & & $\begin{array}{l}3 / 1 / 84- \\
6 / 30 / 84 \\
\end{array}$ & $\begin{array}{l}7 / 1 / 84- \\
7 / 31 / 85\end{array}$ & $\begin{array}{l}8 / 1 / 85- \\
7 / 31 / 86 \\
\end{array}$ & \\
\hline & $\begin{array}{l}\text { Catch } \\
\text { Name }\end{array}$ & Catch Type & Desirability & $\begin{array}{l}\text { Phase } \\
\text { I - } \\
\text { REL }\end{array}$ & $\begin{array}{l}\text { Phase } \\
\text { II - } \\
\text { LN }\end{array}$ & $\begin{array}{l}\text { Phase } \\
\text { III - } \\
\text { BTN }\end{array}$ & $\begin{array}{c}\text { Description / Scientific Name/ } \\
\text { Sample Measurements }\end{array}$ \\
\hline 1 & Albacore & Boney & $\begin{array}{c}\text { High / } \\
\text { Targeted }\end{array}$ & & Y & & Tuna \\
\hline 2 & Anchoveta & Boney & Okay & & & $\mathrm{Y}$ & Anchovies \\
\hline 3 & Angelona & Cartilaginous & Fall Back & $\mathrm{Y}$ & $\mathrm{Y}$ & $\mathrm{Y}$ & \\
\hline 4 & Ayanque & Boney & Okay & & $\mathrm{Y}$ & & $\begin{array}{l}\text { med size drum, Cynoscion } \\
\text { analis, sample: } 32 \mathrm{~cm}, 370 \mathrm{~g}\end{array}$ \\
\hline 5 & Azul & Cartilaginous & Fall Back & $\mathrm{Y}$ & $\mathrm{Y}$ & $\mathrm{Y}$ & \\
\hline 6 & Bonito & Boney & High & $\mathrm{Y}$ & $\mathrm{Y}$ & $\mathrm{Y}$ & $\begin{array}{l}\text { "Pacific bonito", Sarda } \\
\text { chiliensis, sample: } 41 \mathrm{~cm} 600 \mathrm{~g} \\
\text {; } 58 \mathrm{~cm} \text { and } 1700 \mathrm{~g}\end{array}$ \\
\hline 7 & Burro & Boney & Okay & & $\mathrm{Y}$ & & $\begin{array}{l}\text { med size drum, Sciaena } \\
\text { fasciata, sample, } 35 \mathrm{~cm} 850 \mathrm{~g}\end{array}$ \\
\hline 8 & Caballa & Boney & Okay & $\mathrm{Y}$ & $\mathrm{Y}$ & $\mathrm{Y}$ & $\begin{array}{l}\text { "Pacific mackerel", Scomber } \\
\text { japonicus peruanus, sample: } \\
34.5 \mathrm{~cm}, 325 \mathrm{~g}\end{array}$ \\
\hline 9 & Cabinsa & Boney & Okay & & $\mathrm{Y}$ & & \\
\hline 10 & Cabrilla & Boney & Okay & $\mathrm{Y}$ & $\mathrm{Y}$ & $\mathrm{Y}$ & $\begin{array}{l}\text { Peruvian Rock Bass, } \\
\text { Paralabrax humeralis, sample, } \\
28 \mathrm{~cm}, 300 \mathrm{~g}\end{array}$ \\
\hline 11 & Cachema & Boney & Fall Back & & $\mathrm{Y}$ & & \\
\hline 12 & Cangrejo & Crustacean & Fall Back & & $\mathrm{Y}$ & & $\begin{array}{l}\text { "Purple stone Crab", } \\
\text { Platyxanthus orbignyi }\end{array}$ \\
\hline 13 & $\begin{array}{l}\text { Chancho } \\
\text { marino }\end{array}$ & Mammal & Fall Back & $\mathrm{Y}$ & $\mathrm{Y}$ & $\mathrm{Y}$ & Dolphin, "pig of the sea" \\
\hline 14 & Chita & Boney & Okay & & $\mathrm{Y}$ & $\mathrm{Y}$ & $\begin{array}{l}\text { Grunt, Anisotremus scapularis, } \\
\text { sample: } 40 \mathrm{~cm}, 1000 \mathrm{~g}\end{array}$ \\
\hline 15 & Coco & Boney & Okay & $\mathrm{Y}$ & $\mathrm{Y}$ & & med size drum \\
\hline 16 & Cojinova & Boney & $\begin{array}{c}\text { High / } \\
\text { Targeted }\end{array}$ & $\mathrm{Y}$ & & $\mathrm{Y}$ & "blackruff" \\
\hline 17 & Corvina & Boney & $\begin{array}{c}\text { High / } \\
\text { Targeted }\end{array}$ & $\mathrm{Y}$ & $\mathrm{Y}$ & $\mathrm{Y}$ & Drum, Cilus gilberti \\
\hline 18 & Cristalino & Cartilaginous & Fall Back & $\mathrm{Y}$ & $\mathrm{Y}$ & $\mathrm{Y}$ & \\
\hline 19 & Diamante & Cartilaginous & Fall Back & $\mathrm{Y}$ & $\mathrm{Y}$ & & Shark \\
\hline 20 & Guitarra & Cartilaginous & Fall Back & $\mathrm{Y}$ & $\mathrm{Y}$ & $\mathrm{Y}$ & \\
\hline 21 & Jurel & Boney & Okay & $\mathrm{Y}$ & $\mathrm{Y}$ & & $\begin{array}{l}\text { "Chilean Jack Mackerel", } \\
\text { Trachurus symmetricus, } \\
\text { sample } 40 \mathrm{~cm}, 400 \mathrm{~g}\end{array}$ \\
\hline 22 & Langostino & Crustacean & $\begin{array}{c}\text { High / } \\
\text { Targeted }\end{array}$ & $\mathrm{Y}$ & $\mathrm{Y}$ & & Shrimp \\
\hline 23 & Lenguado & Boney & Okay & $\mathrm{Y}$ & $\mathrm{Y}$ & $\mathrm{Y}$ & $\begin{array}{l}\text { "left-eye Flounder", } \\
\text { Paralichthys adspersus, } \\
\text { sample: } 40 \mathrm{~cm}, 750 \mathrm{~g}\end{array}$ \\
\hline 24 & Lisa & Boney & Okay & & $\mathrm{Y}$ & $\mathrm{Y}$ & Mullet \\
\hline 25 & Lorna & Boney & Okay & $\mathrm{Y}$ & $\mathrm{Y}$ & $\mathrm{Y}$ & $\begin{array}{l}\text { med size drum, freq. caught, } \\
\text { Sciaena deliciosa }\end{array}$ \\
\hline 26 & Machete & Boney & Fall Back & & $\mathrm{Y}$ & & not active \\
\hline
\end{tabular}




\begin{tabular}{|c|c|c|c|c|c|c|c|}
\hline & Name & Catch Type & Desirability & $\begin{array}{c}\text { Phase } \\
\text { I - } \\
\text { REL }\end{array}$ & $\begin{array}{l}\text { Phase } \\
\text { II - } \\
\text { LN } \\
\end{array}$ & $\begin{array}{l}\text { Phase } \\
\text { III - } \\
\text { BTN }\end{array}$ & $\begin{array}{l}\text { Description / Scientific } \\
\text { Name/ Sample } \\
\text { Measurements } \\
\end{array}$ \\
\hline 27 & Mantaraya & Cartilaginous & Fall Back & $\mathrm{Y}$ & & & \\
\hline 28 & Mismis & Boney & Okay & $\mathrm{Y}$ & $\mathrm{Y}$ & $\mathrm{Y}$ & \\
\hline 29 & Mojarilla & Boney & Okay & $\mathrm{Y}$ & $\mathrm{Y}$ & & $\begin{array}{l}\text { small drum, Stellifer minor, } \\
\text { sample: } 16 \mathrm{~cm}, 68 \mathrm{~g}\end{array}$ \\
\hline 30 & Pompano & Boney & $\begin{array}{c}\text { High / } \\
\text { Targeted }\end{array}$ & $\mathrm{Y}$ & & & \\
\hline 31 & Pejegallo & Cartilaginous & Fall Back & $\mathrm{Y}$ & $\mathrm{Y}$ & $\mathrm{Y}$ & \\
\hline 32 & Pejerrey & Boney & $\begin{array}{l}\text { High / } \\
\text { Targeted }\end{array}$ & & & Y & $\begin{array}{l}\text { absent from CA in El Nino, } \\
\text { but came strongly in } 1986\end{array}$ \\
\hline 33 & pez dama & Boney & Fall Back & $\mathrm{Y}$ & $\mathrm{Y}$ & & $\begin{array}{l}\text { whale shark, Rhinocodon } \\
\text { typus }\end{array}$ \\
\hline 34 & pez gato & Boney & Fall Back & & $\mathrm{Y}$ & Y & \\
\hline 35 & pez martillo & Cartilaginous & Fall Back & & $\mathrm{Y}$ & & \\
\hline 36 & pez rata & Cartilaginous & Fall Back & $\mathrm{Y}$ & $\mathrm{Y}$ & $\mathrm{Y}$ & \\
\hline 37 & Pinguino & Bird & Fall Back & & $\mathrm{Y}$ & & bird - penguin \\
\hline 38 & Pintadilla & Boney & Okay & $\mathrm{Y}$ & $\mathrm{Y}$ & $\mathrm{Y}$ & $\begin{array}{l}\text { "Morwong", Cheilodactylus } \\
\text { variegatus, eaten by elites } \\
\text { during Later Intermediate, } \\
\text { sample: } 28 \mathrm{~cm}, 300 \mathrm{~g}\end{array}$ \\
\hline 39 & Raya & Cartilaginous & Okay & $\mathrm{Y}$ & $\mathrm{Y}$ & $\mathrm{Y}$ & Ray \\
\hline 40 & Robalo & Boney & $\begin{array}{c}\text { High / } \\
\text { Targeted }\end{array}$ & & $\mathrm{Y}$ & $\mathrm{Y}$ & $\begin{array}{l}\text { Sciaena starksi, largest drum } \\
\text { caught in present day CA, and } \\
\text { Late Int included even larger }\end{array}$ \\
\hline 41 & Sardina & Boney & Okay & & $\mathrm{Y}$ & & Sardine \\
\hline 42 & Sierra & Boney & Okay & $\mathrm{Y}$ & & & $\begin{array}{l}\text { "Sierra mackerel", } \\
\text { Scomberomorus maculatus } \\
\text { sierra, sample, } 44 \mathrm{~cm} \text { and } 450 \mathrm{~g}\end{array}$ \\
\hline 43 & Tembladera & Cartilaginous & Fall Back & $\mathrm{Y}$ & $\mathrm{Y}$ & $\mathrm{Y}$ & "rays", "Discopyge spp." \\
\hline 44 & Tollo & Cartilaginous & Okay & $\mathrm{Y}$ & $\mathrm{Y}$ & $\mathrm{Y}$ & $\begin{array}{l}\text { "Smoothound shark", Mustelus } \\
\text { mento, ranges } 75 \text { to } 150 \mathrm{~cm} \text {, } \\
\text { most common cartilaginous } \\
\text { fish caught in CA today }\end{array}$ \\
\hline 45 & Tortuga & Reptile & Fall Back & & $\mathrm{Y}$ & & Turtle \\
\hline 46 & Trambollo & Boney & Okay & & $\mathrm{Y}$ & & $\begin{array}{l}\text { "Scalled Benny", Labrisomus } \\
\text { philippii, } 28 \mathrm{~cm} 450 \mathrm{~g}\end{array}$ \\
\hline 47 & Volador & Boney & Fall Back & & & Y & flying fish \\
\hline 48 & Zorro & Boney & Okay & $\mathrm{Y}$ & $\mathrm{Y}$ & $\mathrm{Y}$ & $\begin{array}{l}\text { Drum, Menticirrhus rostratus, } \\
\text { sample: } 36 \mathrm{~cm}, 650 \mathrm{~g}\end{array}$ \\
\hline
\end{tabular}

Table 3.2: provides the average catch count for each catch types over all 29 months of the survey. It gives the number of months over which the observations are taken along with the average, standard deviation, and maximum and minimum monthly average. For each of 
these catches there were some months where it was not caught at all. Lorna and Mismis, both found in all three phases, contribute the most to the overall catch count. Likewise, 18 of the 24 most frequent catches are highly desirable or good. The remainders are classified as Fallback. This suggests a rather selective focus towards commercially viable product.

Tables $3.3-3.5$ gives the total catch count for each month based upon the days of the week. Notice that for the highly desirable category, Table 3.3, the week starts heavy and the catches are reduced in size as the week goes on. These are in fact targeted species and appear to be sought after first. Tables 3.4 and 3.5 exhibit the opposite. The number of catches increases in general from Monday through Wednesday, and sometimes there is a second follow up wave beginning on Thursday and going on through Saturday. These suggest that there is a community learning curve where agents learn where other less desirable fish are (2). We suggest that the Fallback category exhibits a similar curve, since if they cannot find a desirable species they will fall back to whatever is available since a successful trip returns with something. 
Table 3.2: The statistics for Catch Count for the entire period.

\begin{tabular}{|l|r|r|r|r|r|}
\hline Species & Obs. & Mean & Std. Dev. & Min & \multicolumn{1}{l|}{ Max } \\
\hline Ayanque & 29 & 17.82759 & 54.45055 & 0 & 276 \\
\hline Azul & 29 & 12.48276 & 21.80207 & 0 & 106 \\
\hline Bonito & 29 & 56.03448 & 158.6428 & 0 & 790 \\
\hline Caballa & 29 & 52.34483 & 142.5926 & 0 & 684 \\
\hline Cabinsa & 29 & 21.72414 & 90.23418 & 0 & 462 \\
\hline Chancho & 29 & 16.72414 & 31.12979 & 0 & 172 \\
\hline marino & 29 & 10.2069 & 26.49445 & 0 & 138 \\
\hline Chita & 29 & 7.413793 & 33.65531 & 0 & 181 \\
\hline Cojinova & 29 & 4.965517 & 10.65861 & 0 & 48 \\
\hline Guitarra & 29 & 94.2069 & 224.9129 & 0 & 840 \\
\hline Jurel & 29 & 256.931 & 758.7817 & 0 & 3114 \\
\hline Langostino & 29 & 27.86207 & 78.90805 & 0 & 328 \\
\hline Lenguado & 29 & 106.7586 & 358.778 & 0 & 1760 \\
\hline Liza & 29 & 14391.24 & 17327.77 & 0 & 62316 \\
\hline Lorna & 29 & 2252 & 3613.489 & 0 & 12672 \\
\hline Mismis & 29 & 24.55172 & 93.20162 & 0 & 480 \\
\hline Mojarilla & 29 & 16.96552 & 91.36211 & 0 & 492 \\
\hline Pompano & 29 & 44.7931 & 63.1316 & 0 & 221 \\
\hline Pejegallo & 29 & 1674.414 & 6201.353 & 0 & 32040 \\
\hline Pejerrey & 29 & 13.72414 & 30.24294 & 0 & 143 \\
\hline Pintadilla & 29 & 142.931 & 162.2372 & 0 & 635 \\
\hline Raya & 14.72414 & 39.03103 & 0 & 200 \\
\hline Tembladera & 179.7241 & 345.7662 & 0 & 1707 \\
\hline Tollo & 41.17241 & 139.9587 & 0 & 666 \\
\hline Volador & 295.6207 & 538.3419 & 0 & 2304 \\
\hline Zorro & & & & \\
\hline & 29 & & 0 & 0 \\
\hline
\end{tabular}


Figure 3.1 provides a graphical illustration of how catch count changes monthly over the observation period. The red strip represents the size of catches caught near Cerro Azul proper (Cerro Azul, La Centinela, and El Faro). The blue strip corresponds to the total caught south of Cerro Azul, and the red band stands for those catches made on trips north of Cerro Azul. This catch is present in all three phases, but particularly targeted in the La Nina phase. Appendix A, which will be added later, contains these graphs for all of the recorded species.

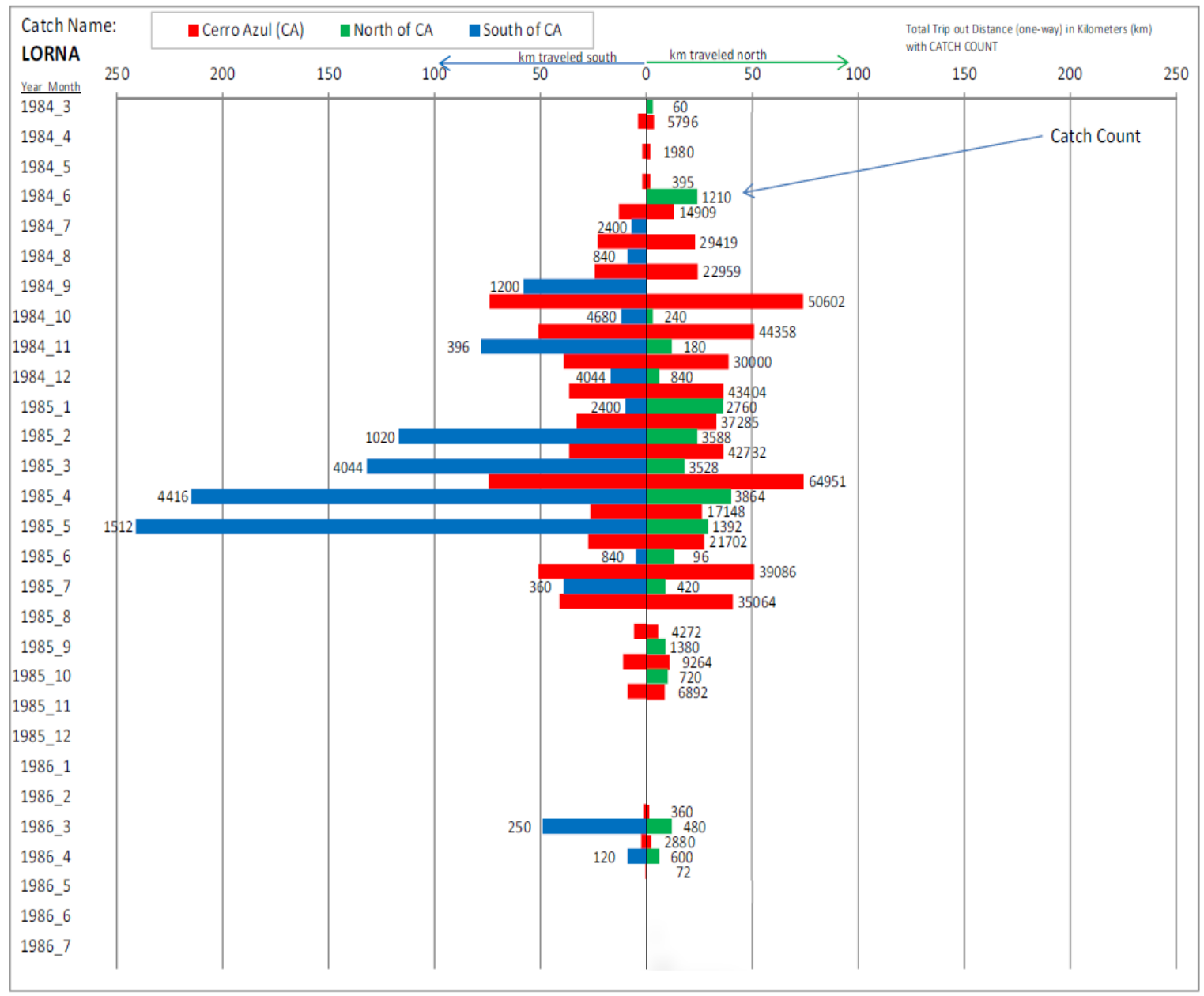

Figure 3.1: The Total catch count per month relative to trip direction for: Lorna 


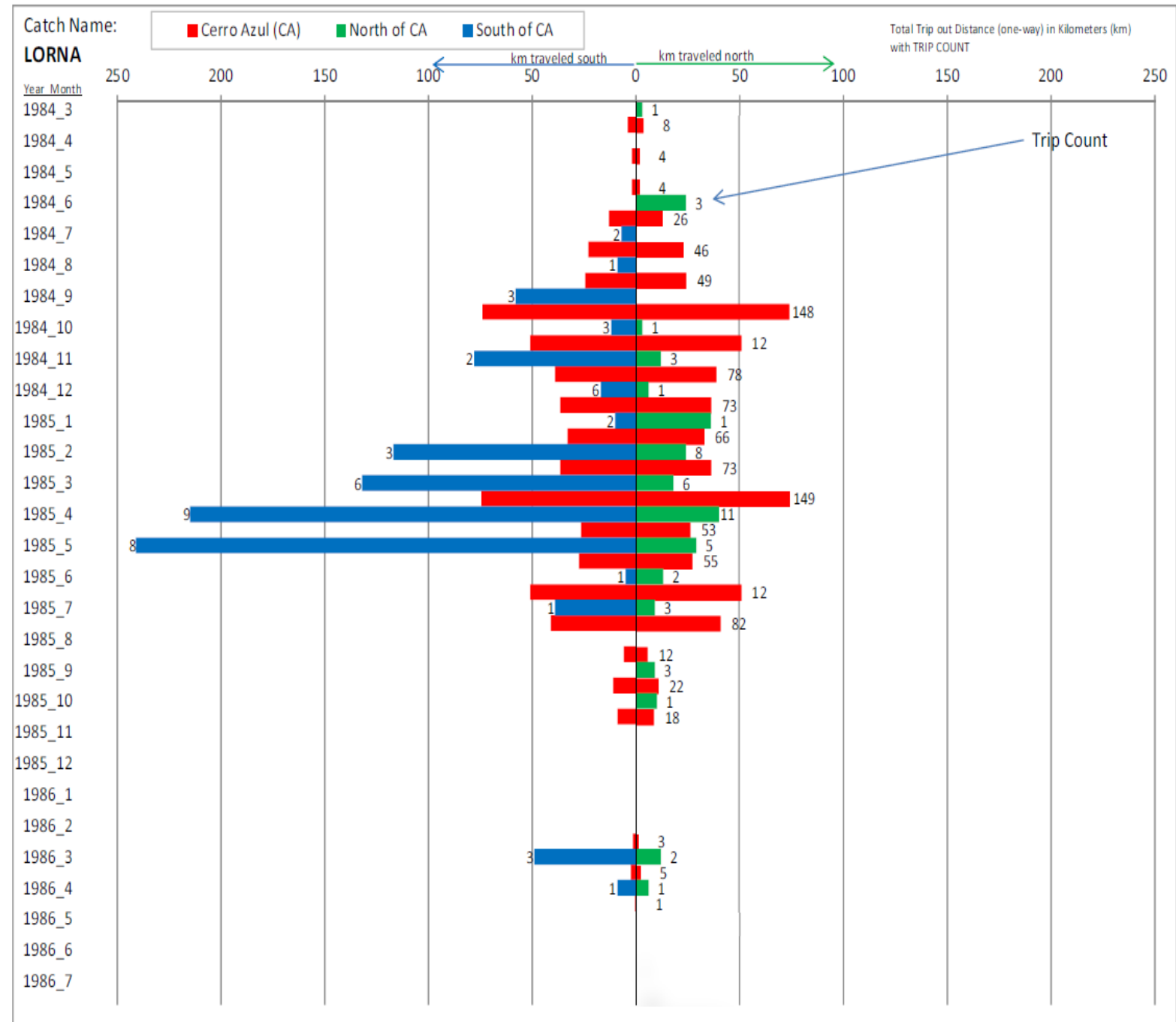

Figure 3.2: The Total trip count per month relative to trip direction for Lorna 
Table 3.3: Total Catch Count by month for High Desirability.

\begin{tabular}{|c|c|c|c|c|c|c|c|c|c|}
\hline \multicolumn{10}{|c|}{ High Desirability } \\
\hline Year & Month & Desire. & Mon & Tues & Wed & Thur & Fri & Sat & Sun \\
\hline 1984 & 3 & 1 & 166 & 789 & 1073 & 1235 & 644 & 746 & 327 \\
\hline 1984 & 4 & 1 & 3442 & 567 & 762 & 625 & 1466 & 960 & 934 \\
\hline 1984 & 5 & 1 & 47 & 180 & 1039 & 258 & 531 & 2 & 35 \\
\hline 1984 & 6 & 1 & & & 20 & 7 & & & \\
\hline \multicolumn{3}{|c|}{$\begin{array}{c}\text { End of Residual EI Nino } \\
\text { (SUM) }\end{array}$} & 3655 & 1536 & 2894 & 2125 & 2641 & 1708 & 1296 \\
\hline 1984 & 7 & & & & & & & & \\
\hline 1984 & 8 & 1 & & 8 & & 214 & 434 & 396 & 347 \\
\hline 1984 & 9 & 1 & 144 & 5 & 17 & & & 17 & 40 \\
\hline 1984 & 10 & 1 & & & & & & 18 & \\
\hline 1984 & 11 & & & & & & & & \\
\hline 1984 & 12 & 1 & 13 & 24 & & & 20 & 12 & \\
\hline 1985 & 1 & 1 & 54 & & 9 & 25 & 20 & 6 & 9 \\
\hline 1985 & 2 & 1 & 8 & 60 & 580 & 88 & 94 & 428 & 146 \\
\hline 1985 & 3 & & & & & & & & \\
\hline 1985 & 4 & 1 & & & 75 & & & 159 & 130 \\
\hline 1985 & 5 & 1 & 11 & & & & 4 & & 65 \\
\hline 1985 & 6 & & & & & & & & \\
\hline 1985 & 7 & & & & & & & & \\
\hline \multicolumn{3}{|c|}{ End of La Nina (SUM) } & 230 & 97 & 681 & 327 & 572 & 1036 & 737 \\
\hline 1985 & 8 & & & & & & & & \\
\hline 1985 & 9 & 1 & & 13 & 13 & & & 3 & \\
\hline 1985 & 10 & & & & & & & & \\
\hline 1985 & 11 & 1 & & 15 & 24 & 150 & 3 & & \\
\hline 1985 & 12 & & & & & & & & \\
\hline 1986 & 1 & 1 & & & & & 12 & & \\
\hline 1986 & 2 & 1 & & & & & & 9 & \\
\hline 1986 & 3 & & & & & & & & \\
\hline 1986 & 4 & 1 & & 10 & 9 & & & 10 & \\
\hline 1986 & 5 & & & & & & & & \\
\hline 1986 & 6 & 1 & & & & & & 12 & \\
\hline 1986 & 7 & 1 & 263 & 116 & 77 & 42 & 12 & 293 & 88 \\
\hline \multicolumn{3}{|c|}{ End of Back to Normal (SUM) } & 263 & 154 & 123 & 192 & 27 & 327 & 88 \\
\hline
\end{tabular}


Table 3.4: Total Catch count by month for Okay Desirability.

\begin{tabular}{|c|c|c|c|c|c|c|c|c|c|}
\hline \multicolumn{10}{|c|}{ Sum of All Fish Caught for each month on each Day of week using Return Day } \\
\hline \multicolumn{10}{|c|}{ Okay Desirabilty } \\
\hline Year & Month & Desire. & Mon & Tues & Wed & Thur & Fri & Sat & Sun \\
\hline 1984 & 3 & 2 & 542 & 820 & 2059 & 897 & 789 & 4210 & 1208 \\
\hline 1984 & 4 & 2 & 780 & 1211 & 1059 & 432 & 244 & 1102 & 242 \\
\hline 1984 & 5 & 2 & 2 & 424 & 347 & 5220 & 558 & 24 & 84 \\
\hline 1984 & 6 & 2 & 2849 & 1189 & 2067 & 928 & 5161 & 5539 & 183 \\
\hline \multicolumn{3}{|c|}{$\begin{array}{c}\text { End of Residual EI Nino } \\
\text { (SUM) }\end{array}$} & 4173 & 3644 & 5532 & 7477 & 6752 & 10875 & 1717 \\
\hline 1984 & 7 & 2 & 3961 & 3845 & 8179 & 4006 & 2202 & 3401 & 7937 \\
\hline 1984 & 8 & 2 & 2986 & 1941 & 3732 & 8215 & 5362 & 5065 & 1660 \\
\hline 1984 & 9 & 2 & 8491 & 9944 & 6363 & 4385 & 7753 & 12981 & 11187 \\
\hline 1984 & 10 & 2 & 5233 & 8018 & 17564 & 12289 & 9322 & 9288 & 6973 \\
\hline 1984 & 11 & 2 & 3673 & 7202 & 10158 & 12070 & 6226 & 7466 & 5702 \\
\hline 1984 & 12 & 2 & 9351 & 8523 & 14697 & 4977 & 4323 & 10897 & 8299 \\
\hline 1985 & 1 & 2 & 9659 & 10406 & 8038 & 4942 & 6574 & 11701 & 10940 \\
\hline 1985 & 2 & 2 & 3205 & 5522 & 10034 & 8045 & 11990 & 10749 & 8719 \\
\hline 1985 & 3 & 2 & 10853 & 7938 & 12474 & 5744 & 11174 & 15839 & 12167 \\
\hline 1985 & 4 & 2 & 6236 & 5339 & 3445 & 4018 & 3565 & 4965 & 5994 \\
\hline 1985 & 5 & 2 & 2058 & 3671 & 5099 & 4905 & 9210 & 5022 & 5544 \\
\hline 1985 & 6 & 2 & 4140 & 5280 & 9713 & 10348 & 7802 & 4810 & 3326 \\
\hline 1985 & 7 & 2 & 2388 & 4872 & 4740 & 7632 & 6636 & 4884 & 4896 \\
\hline \multicolumn{3}{|c|}{ End of La Nina (SUM) } & 72234 & 82501 & 114236 & 91576 & 92139 & 107068 & 93344 \\
\hline 1985 & 8 & 2 & 720 & 480 & 180 & 420 & 1812 & 660 & \\
\hline 1985 & 9 & 2 & 1988 & 11 & 2311 & 1353 & 2784 & 2606 & 730 \\
\hline 1985 & 10 & 2 & 960 & 1202 & 1988 & 1429 & 1004 & 817 & 1767 \\
\hline 1985 & 11 & 2 & 14 & 44 & 536 & 3730 & 842 & 1110 & 92 \\
\hline 1985 & 12 & 2 & 248 & 207 & 411 & 337 & 307 & 63 & 4 \\
\hline 1986 & 1 & 2 & 691 & & & 384 & 83 & & \\
\hline 1986 & 2 & 2 & 2 & 673 & 35 & 1258 & 2305 & 216 & \\
\hline 1986 & 3 & 2 & 738 & 456 & 9315 & 2528 & 465 & 1700 & 565 \\
\hline 1986 & 4 & 2 & 79 & 360 & 4 & 23 & 97 & 882 & 14 \\
\hline 1986 & 5 & 2 & 17 & & 14 & 2 & 5 & & 4 \\
\hline 1986 & 6 & 2 & 2 & 6 & & 10 & 2 & & 4 \\
\hline 1986 & 7 & 2 & & & 12 & 2 & 4 & & 1 \\
\hline End o & $\begin{array}{l}\text { Back to I } \\
\text { (SUM) }\end{array}$ & rmal & 5459 & 3439 & 14806 & 11476 & 9710 & 8054 & 3181 \\
\hline
\end{tabular}


Table 3.5: Total Catch count by month for Fall Back Desirability

\begin{tabular}{|c|c|c|c|c|c|c|c|c|c|}
\hline \multicolumn{10}{|c|}{ Fall Back Desirability } \\
\hline Year & Month & Desire. & Mon & Tues & Wed & Thur & Fri & Sat & Sun \\
\hline 1984 & 3 & 3 & 5 & 16 & 94 & 54 & 82 & 292 & 58 \\
\hline 1984 & 4 & 3 & 8 & 55 & 241 & 311 & 218 & 63 & 42 \\
\hline 1984 & 5 & 3 & 2 & 26 & 88 & 142 & 103 & 506 & 16 \\
\hline 1984 & 6 & 3 & 7 & 51 & 134 & 58 & 134 & 150 & 18 \\
\hline \multicolumn{3}{|c|}{$\begin{array}{c}\text { End of Residual EI Nino } \\
\text { (SUM) }\end{array}$} & 22 & 148 & 557 & 565 & 537 & 1011 & 134 \\
\hline 1984 & 7 & 3 & 3 & & 33 & & 47 & 3 & 85 \\
\hline 1984 & 8 & 3 & 62 & 68 & 173 & 133 & 48 & 52 & 120 \\
\hline 1984 & 9 & 3 & 70 & 103 & 241 & 178 & 168 & 261 & 228 \\
\hline 1984 & 10 & 3 & 251 & 88 & 309 & 175 & 261 & 164 & 140 \\
\hline 1984 & 11 & 3 & 175 & 152 & 457 & 337 & 396 & 512 & 327 \\
\hline 1984 & 12 & 3 & 610 & 39 & 129 & 297 & 383 & 107 & 415 \\
\hline 1985 & 1 & 3 & 42 & 115 & 125 & 104 & 72 & 748 & 90 \\
\hline 1985 & 2 & 3 & 30 & 76 & 51 & 174 & 409 & 204 & 199 \\
\hline 1985 & 3 & 3 & 135 & 186 & 196 & 122 & 265 & 203 & 453 \\
\hline 1985 & 4 & 3 & 116 & 269 & 173 & 43 & 101 & 89 & 201 \\
\hline 1985 & 5 & 3 & 44 & 13 & 438 & 292 & 651 & 310 & 222 \\
\hline 1985 & 6 & 3 & & 70 & 254 & 119 & 94 & 182 & 62 \\
\hline 1985 & 7 & 3 & & 6 & 57 & 5 & 5 & & \\
\hline \multicolumn{3}{|c|}{ End of La Nina (SUM) } & 1538 & 1185 & 2636 & 1979 & 2900 & 2835 & 2542 \\
\hline 1985 & 8 & 3 & & & & & & & \\
\hline 1985 & 9 & 3 & & & & 24 & 16 & 30 & 10 \\
\hline 1985 & 10 & 3 & 29 & 15 & 38 & 9 & 67 & 82 & 37 \\
\hline 1985 & 11 & 3 & 34 & 208 & 172 & 88 & 212 & 81 & 16 \\
\hline 1985 & 12 & 3 & 27 & 41 & 119 & 14 & 21 & 81 & 176 \\
\hline 1986 & 1 & 3 & 26 & & 4 & & 18 & 10 & 1 \\
\hline 1986 & 2 & 3 & 31 & 211 & 291 & 112 & 138 & 19 & 35 \\
\hline 1986 & 3 & 3 & 424 & 133 & 3554 & 329 & 913 & 1163 & 452 \\
\hline 1986 & 4 & 3 & 181 & 557 & 135 & 855 & 1301 & 111 & 14 \\
\hline 1986 & 5 & 3 & 6 & 924 & 47 & 168 & 125 & 234 & 88 \\
\hline 1986 & 6 & 3 & 2586 & 2015 & 5185 & 6359 & 1309 & 6433 & 1380 \\
\hline 1986 & 7 & 3 & 8722 & 8704 & 2835 & 6088 & 3146 & 8181 & 2766 \\
\hline \multicolumn{3}{|c|}{ End of Back to Normal(SUM) } & 12066 & 12808 & 12380 & 14046 & 7266 & 16425 & 4975 \\
\hline
\end{tabular}




\subsection{EFFORT EXPENDED ON TRIPS OVER TIME}

It is clear from the above, that catch quality is a factor in the selection of a fishing trip by agents. In this section we provide evidence relative to the importance of another concern, resource expenditure. All of the trips in the database are successful trips in the sense they came back with something. We view the distance travelled to achieve that success are an investment in the basic fishing activity. So the Payout, or catch count, can be viewed as a return on the investment.

The following table, Table 3.6, shows the distance from Cerro Azul to all other sites. That was visited during the entire survey period. Notice that sites 17 a-c are all viewed to be co-located and constitute the immediate area around Cerro Azul. We assume that the round trip distance to each is one $\mathrm{km}$. 
Table 3.6: The distance from Cerro Azul to all sites

\begin{tabular}{|c|c|c|c|c|}
\hline & Site Name & $\begin{array}{c}\text { One Way } \\
\text { Distance }(\mathrm{KM})\end{array}$ & $\begin{array}{l}\text { Round Trip } \\
\text { Dist. (KM) }\end{array}$ & $\begin{array}{l}\text { Relative to } \\
\text { Cerro Azul } \\
\end{array}$ \\
\hline 1 & San Bartolo & 78 & 156 & North \\
\hline 2 & San Pedro & 66 & 132 & North \\
\hline 3 & Cerro Blanco & 54 & 108 & North \\
\hline 4 & Mala & 44 & 88 & North \\
\hline 5 & Playa Asia & 33 & 66 & North \\
\hline 6 & Isla Asia & 31 & 62 & North \\
\hline 7 & Asia & 28 & 56 & North \\
\hline 8 & Mal Paso & 25 & 50 & North \\
\hline 9 & Playa Sarapampa & 23 & 46 & North \\
\hline 10 & Valdivia & 18 & 36 & North \\
\hline 11 & Los Leones & 16 & 32 & North \\
\hline 12 & Isla Corriente & 10 & 20 & North \\
\hline 13 & Corriente & 9 & 18 & North \\
\hline 14 & Gallardo & 7 & 14 & North \\
\hline 15 & Los Lobos & 6 & 12 & North \\
\hline 16 & Los Reyes & 3 & 6 & North \\
\hline $17 \mathrm{a}$ & Cerro Azul & 0.5 & 1 & Cerro Azul \\
\hline $17 \mathrm{~b}$ & Faro & 0.5 & 1 & Cerro Azul \\
\hline $17 \mathrm{c}$ & La Centinela & 0.5 & 1 & Cerro Azul \\
\hline 18 & Puente Tabla & 2 & 4 & South \\
\hline 19 & Playa La Costa & 3 & 6 & South \\
\hline 20 & Santa Bárbara & 5 & 10 & South \\
\hline 21 & $\begin{array}{l}\text { San Vicente de } \\
\text { Canete }\end{array}$ & 9 & 18 & South \\
\hline 22 & Playa Hermosa & 10 & 20 & South \\
\hline 23 & Boca del Rio & 14 & 28 & South \\
\hline 24 & Herbay & 16 & 32 & South \\
\hline 25 & Cinco Cruces & 28 & 56 & South \\
\hline 26 & Colorado & 39 & 78 & South \\
\hline 27 & Jaway & 40 & 80 & South \\
\hline 28 & Tambo de Mora & 58 & 116 & South \\
\hline 29 & Monzo & 99 & 198 & South \\
\hline
\end{tabular}


Table 3.7 gives the number of trips that returned on a given day of the week over the entire period of the survey relative to each of the catch types. Lorna and Mismis taken together constitute over a third of the overall number trips in the period. One reason for that is they are present in all three phases and therefore can be a target more often than some others [20]. Note that the overall number of trips increases from Monday through to Saturday. This may result from the fact that as more information is acquired about the available catches, there is more opportunity to target a particular catch and location. 
Table 3.7: Trip Count over Entire Period Based upon Return Date.

\begin{tabular}{|c|c|c|c|c|c|c|c|c|}
\hline Catch & Sunday & Monday & Tuesday & Wednesday & Thursday & Friday & Saturday & TOTAL \\
\hline albacora & 1 & 0 & 0 & 0 & 0 & 0 & & 1 \\
\hline anchoveta & & 0 & 0 & 1 & 1 & 0 & & 2 \\
\hline angelona & & 1 & 1 & 3 & 3 & 0 & 1 & 9 \\
\hline ayanque & 1 & 5 & 2 & 0 & 3 & 5 & 6 & 22 \\
\hline azul & 29 & 21 & 21 & 50 & 32 & 41 & 40 & 234 \\
\hline bonito & 27 & 16 & 13 & 10 & 22 & 18 & 27 & 133 \\
\hline burro & & 0 & 0 & 1 & 1 & 0 & & 2 \\
\hline caballa & 3 & 1 & 3 & 2 & 5 & 5 & 3 & 22 \\
\hline cabinsa & 1 & 0 & 0 & 0 & 0 & 2 & 1 & 4 \\
\hline cabrilla & 1 & 0 & 0 & 1 & 1 & 1 & 1 & 5 \\
\hline cachema & & 0 & 0 & 0 & 0 & 1 & & 1 \\
\hline camarón & & 0 & 0 & 3 & 0 & 1 & & 4 \\
\hline cangrejo & & 0 & 0 & 1 & 0 & 0 & & 1 \\
\hline chancho & 66 & 37 & 64 & 94 & 69 & 88 & 80 & 498 \\
\hline chita & 6 & 2 & 11 & 7 & 9 & 12 & 9 & 56 \\
\hline coco & 2 & 1 & 2 & 0 & 1 & 3 & 1 & 10 \\
\hline cojinova & 8 & 7 & 10 & 5 & 2 & 2 & 12 & 46 \\
\hline corvina & 2 & 0 & 2 & 1 & 2 & 2 & 2 & 11 \\
\hline cristalino & 1 & 7 & 2 & 8 & 9 & 5 & 12 & 44 \\
\hline diamante & 2 & 2 & 2 & 5 & 0 & 2 & 4 & 17 \\
\hline guitarra & 6 & 5 & 20 & 16 & 21 & 16 & 13 & 97 \\
\hline jurel & 2 & 1 & 5 & 3 & 3 & 5 & 3 & 22 \\
\hline langostino & 7 & 8 & 11 & 17 & 13 & 10 & 7 & 73 \\
\hline lenguado & 7 & 6 & 14 & 6 & 8 & 16 & 10 & 67 \\
\hline lisa & 1 & 3 & 2 & 1 & 3 & 3 & 2 & 15 \\
\hline lorna & 180 & 132 & 160 & 201 & 197 & 208 & 213 & 1291 \\
\hline machete & & 1 & 0 & 1 & 0 & 0 & 1 & 3 \\
\hline mantaraya & & 0 & 0 & 0 & 0 & 1 & & 1 \\
\hline mismis & 126 & 92 & 113 & 150 & 137 & 155 & 150 & 923 \\
\hline mojarilla & & 0 & 1 & 0 & 1 & 2 & 3 & 7 \\
\hline pampano & & 0 & 2 & 1 & 5 & 2 & 2 & 12 \\
\hline pejegallo & 28 & 16 & 28 & 38 & 34 & 37 & 36 & 217 \\
\hline pejerrey & 9 & 12 & 12 & 16 & 20 & 11 & 18 & 98 \\
\hline pez dama & 1 & 0 & 1 & 1 & 0 & 1 & & 4 \\
\hline pez gato & 2 & 0 & 0 & 2 & 0 & 1 & 1 & 6 \\
\hline pez & 2 & 1 & 4 & 2 & 0 & 3 & 1 & 13 \\
\hline pez rata & 5 & 1 & 6 & 12 & 3 & 5 & 10 & 42 \\
\hline pinguino & & 0 & 1 & 0 & 0 & 0 & & 1 \\
\hline pintadilla & & 1 & 5 & 2 & 5 & 6 & 5 & 24 \\
\hline raya & 120 & 56 & 92 & 149 & 166 & 175 & 146 & 904 \\
\hline robalo & & 0 & 0 & 1 & 1 & 0 & & 2 \\
\hline sardina & 1 & 1 & 1 & 1 & 0 & 0 & & 4 \\
\hline sierra & 1 & 1 & 0 & 2 & 1 & 1 & & 6 \\
\hline tembladera & 1 & 0 & 4 & 7 & 5 & 7 & 2 & 26 \\
\hline tollo & 122 & 83 & 82 & 141 & 158 & 178 & 163 & 927 \\
\hline tortuga & & 0 & 0 & 0 & 0 & 1 & 1 & 2 \\
\hline trambollo & & 1 & 0 & 0 & 0 & 0 & & 1 \\
\hline volador & 3 & 2 & 0 & 2 & 0 & 0 & 2 & 9 \\
\hline zorro & 10 & 12 & 8 & 16 & 13 & 18 & 17 & 94 \\
\hline Total & 784 & 535 & 705 & 980 & 954 & 1050 & 1005 & 6013 \\
\hline
\end{tabular}


Table 3.8 now breaks down the trips for each catch type into each of the three phases. Now we can see why the trip count increases over the course of the week. Certain highly targeted and desirable catches are the subject of increasingly more trips during the course of the week. For example, the number of trips that caught Lorna, a staple in the fishing repertoire, increases in general over the course of the week in all three phases. It is clearly a continuing target for fishermen and the fact that the number of trips increases over the course of a week reflects the "learning curve" that we mentioned earlier. In this case the curve extends across all days of the week as opposed to others that extend over earlier portions of the week, Monday through Wednesday for example. 
Table 3.8: Catches by Phase for Entire Period using Return Date

\begin{tabular}{|c|c|c|c|c|c|c|c|c|c|}
\hline \multicolumn{10}{|c|}{ Phase $1=$ Residual EI Nino, 2 = La Nina, 3 = Back to Normal } \\
\hline Catches & Phase & Sunday & Monday & Tuesday & Wed. & Thursday & Friday & Saturday & Total \\
\hline albacora & 1 & 0 & 0 & 0 & 0 & 0 & 0 & 0 & 0 \\
\hline albacora & 2 & 1 & 0 & 0 & 0 & 0 & 0 & 0 & 1 \\
\hline albacora & 3 & 0 & 0 & 0 & 0 & 0 & 0 & 0 & 0 \\
\hline anchoveta & 1 & 0 & 0 & 0 & 0 & 1 & 0 & 0 & 1 \\
\hline anchoveta & 2 & 0 & 0 & 0 & 0 & 0 & 0 & 0 & 0 \\
\hline anchoveta & 3 & 0 & 0 & 0 & 1 & 0 & 0 & 0 & 1 \\
\hline angelona & 1 & 0 & 0 & 0 & 0 & 1 & 0 & 0 & 1 \\
\hline angelona & 2 & 0 & 1 & 1 & 2 & 2 & 0 & 1 & 7 \\
\hline angelona & 3 & 0 & 0 & 0 & 1 & 0 & 0 & 0 & 1 \\
\hline ayanque & 1 & 0 & 0 & 0 & 0 & 0 & 0 & 0 & 0 \\
\hline ayanque & 2 & 1 & 5 & 2 & 0 & 3 & 5 & 6 & 22 \\
\hline ayanque & 3 & 0 & 0 & 0 & 0 & 0 & 0 & 0 & 0 \\
\hline azul & 1 & 4 & 0 & 3 & 7 & 9 & 8 & 7 & 38 \\
\hline azul & 2 & 14 & 9 & 5 & 18 & 9 & 12 & 13 & 80 \\
\hline azul & 3 & 11 & 12 & 13 & 25 & 14 & 21 & 20 & 116 \\
\hline bonito & 1 & 6 & 8 & 7 & 4 & 3 & 1 & 4 & 33 \\
\hline bonito & 2 & 21 & 8 & 4 & 5 & 12 & 15 & 22 & 87 \\
\hline bonito & 3 & 0 & 0 & 2 & 1 & 7 & 2 & 1 & 13 \\
\hline burro & 1 & 0 & 0 & 0 & 0 & 0 & 0 & 0 & 0 \\
\hline burro & 2 & 0 & 0 & 0 & 1 & 1 & 0 & 0 & 2 \\
\hline burro & 3 & 0 & 0 & 0 & 0 & 0 & 0 & 0 & 0 \\
\hline caballa & 1 & 0 & 0 & 2 & 0 & 2 & 1 & 1 & 6 \\
\hline caballa & 2 & 2 & 1 & 1 & 0 & 3 & 4 & 1 & 12 \\
\hline caballa & 3 & 1 & 0 & 0 & 2 & 0 & 0 & 1 & 4 \\
\hline cabinsa & 1 & 0 & 0 & 0 & 0 & 0 & 0 & 0 & 0 \\
\hline cabinsa & 2 & 1 & 0 & 0 & 0 & 0 & 2 & 1 & 4 \\
\hline cabinsa & 3 & 0 & 0 & 0 & 0 & 0 & 0 & 0 & 0 \\
\hline cabrilla & 1 & 0 & 0 & 0 & 0 & 0 & 1 & 0 & 1 \\
\hline cabrilla & 2 & 1 & 0 & 0 & 0 & 1 & 0 & 1 & 3 \\
\hline cabrilla & 3 & 0 & 0 & 0 & 1 & 0 & 0 & 0 & 1 \\
\hline cachema & 1 & 0 & 0 & 0 & 0 & 0 & 0 & 0 & 0 \\
\hline cachema & 2 & 0 & 0 & 0 & 0 & 0 & 1 & 0 & 1 \\
\hline cachema & 3 & 0 & 0 & 0 & 0 & 0 & 0 & 0 & 0 \\
\hline camarón & 1 & 0 & 0 & 0 & 3 & 0 & 1 & 0 & 4 \\
\hline camarón & 2 & 0 & 0 & 0 & 0 & 0 & 0 & 0 & 0 \\
\hline camarón & 3 & 0 & 0 & 0 & 0 & 0 & 0 & 0 & 0 \\
\hline cangrejo & 1 & 0 & 0 & 0 & 0 & 0 & 0 & 0 & 0 \\
\hline
\end{tabular}




\begin{tabular}{|c|c|c|c|c|c|c|c|c|c|}
\hline Species & Phase & Sunday & Monday & Tuesday & Wed. & Thursday & Friday & Saturday & Total \\
\hline cangrejo & 2 & 0 & 0 & 0 & 1 & 0 & 0 & 0 & 1 \\
\hline cangrejo & 3 & 0 & 0 & 0 & 0 & 0 & 0 & 0 & 0 \\
\hline $\begin{array}{l}\text { chancho } \\
\text { marino }\end{array}$ & 1 & 2 & 3 & 6 & 15 & 9 & 8 & 11 & 54 \\
\hline $\begin{array}{l}\text { chancho } \\
\text { marino }\end{array}$ & 2 & 51 & 25 & 33 & 50 & 40 & 49 & 46 & 294 \\
\hline $\begin{array}{l}\text { chancho } \\
\text { marino }\end{array}$ & 3 & 13 & 9 & 25 & 29 & 20 & 31 & 23 & 150 \\
\hline chita & 1 & 0 & 0 & 0 & 0 & 0 & 0 & 0 & 0 \\
\hline chita & 2 & 5 & 2 & 8 & 6 & 9 & 9 & 6 & 45 \\
\hline chita & 3 & 1 & 0 & 3 & 1 & 0 & 3 & 3 & 11 \\
\hline coco & 1 & 2 & 0 & 2 & 0 & 1 & 3 & 1 & 9 \\
\hline coco & 2 & 0 & 1 & 0 & 0 & 0 & 0 & 0 & 1 \\
\hline coco & 3 & 0 & 0 & 0 & 0 & 0 & 0 & 0 & 0 \\
\hline cojinova & 1 & 0 & 0 & 0 & 0 & 0 & 1 & 0 & 1 \\
\hline cojinova & 2 & 0 & 0 & 0 & 0 & 0 & 0 & 0 & 0 \\
\hline cojinova & 3 & 8 & 7 & 10 & 5 & 2 & 1 & 12 & 45 \\
\hline corvina & 1 & 0 & 0 & 0 & 0 & 1 & 1 & 1 & 3 \\
\hline corvina & 2 & 1 & 0 & 1 & 0 & 0 & 1 & 0 & 3 \\
\hline corvina & 3 & 1 & 0 & 1 & 1 & 1 & 0 & 1 & 5 \\
\hline cristalino & 1 & 0 & 0 & 0 & 0 & 1 & 0 & 1 & 2 \\
\hline cristalino & 2 & 1 & 7 & 2 & 6 & 7 & 5 & 11 & 39 \\
\hline cristalino & 3 & 0 & 0 & 0 & 2 & 1 & 0 & 0 & 3 \\
\hline diamante & 1 & 1 & 0 & 0 & 0 & 0 & 0 & 1 & 2 \\
\hline diamante & 2 & 1 & 2 & 2 & 5 & 0 & 2 & 3 & 15 \\
\hline diamante & 3 & 0 & 0 & 0 & 0 & 0 & 0 & 0 & 0 \\
\hline guitarra & 1 & 2 & 2 & 8 & 6 & 10 & 12 & 7 & 47 \\
\hline guitarra & 2 & 3 & 2 & 1 & 3 & 2 & 0 & 3 & 14 \\
\hline guitarra & 3 & 1 & 1 & 11 & 7 & 9 & 4 & 3 & 36 \\
\hline jurel & 1 & 2 & 1 & 5 & 2 & 2 & 3 & 3 & 18 \\
\hline jurel & 2 & 0 & 0 & 0 & 1 & 1 & 2 & 0 & 4 \\
\hline jurel & 3 & 0 & 0 & 0 & 0 & 0 & 0 & 0 & 0 \\
\hline langostino & 1 & 6 & 8 & 11 & 16 & 13 & 10 & 5 & 69 \\
\hline langostino & 2 & 1 & 0 & 0 & 1 & 0 & 0 & 2 & 4 \\
\hline langostino & 3 & 0 & 0 & 0 & 0 & 0 & 0 & 0 & 0 \\
\hline lenguado & 1 & 6 & 3 & 8 & 4 & 5 & 9 & 3 & 38 \\
\hline lenguado & 2 & 1 & 3 & 6 & 2 & 2 & 7 & 7 & 28 \\
\hline lenguado & 3 & 0 & 0 & 0 & 0 & 1 & 0 & 0 & 1 \\
\hline lisa & 1 & 0 & 0 & 0 & 0 & 0 & 0 & 0 & 0 \\
\hline lisa & 2 & 0 & 0 & 0 & 0 & 1 & 1 & 0 & 2 \\
\hline lisa & 3 & 1 & 3 & 2 & 1 & 2 & 2 & 2 & 13 \\
\hline lorna & 1 & 3 & 5 & 3 & 6 & 6 & 13 & 10 & 46 \\
\hline
\end{tabular}




\begin{tabular}{|c|c|c|c|c|c|c|c|c|c|}
\hline Species & Phase & Sunday & Monday & Tuesday & Wed. & Thursday & Friday & Saturday & Total \\
\hline lorna & 2 & 172 & 120 & 152 & 185 & 178 & 175 & 191 & 1173 \\
\hline lorna & 3 & 5 & 7 & 5 & 10 & 13 & 20 & 12 & 72 \\
\hline machete & 1 & 0 & 0 & 0 & 0 & 0 & 0 & 0 & 0 \\
\hline machete & 2 & 0 & 1 & 0 & 1 & 0 & 0 & 1 & 3 \\
\hline machete & 3 & 0 & 0 & 0 & 0 & 0 & 0 & 0 & 0 \\
\hline mantaraya & 1 & 0 & 0 & 0 & 0 & 0 & 1 & 0 & 1 \\
\hline mantaraya & 1 & 0 & 0 & 0 & 0 & 0 & 0 & 0 & 0 \\
\hline mantaraya & 1 & 0 & 0 & 0 & 0 & 0 & 0 & 0 & 0 \\
\hline mismis & 1 & 2 & 0 & 1 & 0 & 1 & 6 & 1 & 11 \\
\hline mismis & 2 & 122 & 92 & 112 & 148 & 133 & 144 & 144 & 895 \\
\hline mismis & 3 & 2 & 0 & 0 & 2 & 3 & 5 & 5 & 17 \\
\hline mojarilla & 1 & 0 & 0 & 0 & 0 & 0 & 1 & 0 & 1 \\
\hline mojarilla & 2 & 0 & 0 & 1 & 0 & 1 & 1 & 3 & 6 \\
\hline mojarilla & 3 & 0 & 0 & 0 & 0 & 0 & 0 & 0 & $\underline{0}$ \\
\hline pampano & 1 & 0 & 0 & 2 & 1 & 5 & 2 & 2 & 12 \\
\hline pampano & 2 & 0 & 0 & 0 & 0 & 0 & 0 & 0 & 0 \\
\hline pampano & 3 & 0 & 0 & 0 & 0 & 0 & 0 & 0 & 0 \\
\hline pejegallo & 1 & 0 & 1 & 2 & 7 & 6 & 4 & 3 & 23 \\
\hline pejegallo & 2 & 23 & 13 & 20 & 26 & 22 & 27 & 28 & 159 \\
\hline pejegallo & 3 & 5 & 2 & 6 & 5 & 6 & 6 & 5 & 35 \\
\hline pejerrey & 1 & 0 & 0 & 0 & 0 & 0 & 0 & 0 & 0 \\
\hline pejerrey & 2 & 0 & 0 & 0 & 0 & 0 & 0 & 0 & 0 \\
\hline pejerrey & 3 & 9 & 12 & 12 & 16 & 20 & 11 & 18 & 98 \\
\hline pez dama & 1 & 0 & 0 & 1 & 0 & 0 & 0 & 0 & 1 \\
\hline pez dama & 2 & 1 & 0 & 0 & 1 & 0 & 1 & 0 & 3 \\
\hline pez dama & 3 & 0 & 0 & 0 & 0 & 0 & 0 & 0 & 0 \\
\hline pez gato & 1 & 0 & 0 & 0 & 0 & 0 & 0 & 0 & 0 \\
\hline pez gato & 2 & 2 & 0 & 0 & 0 & 0 & 1 & 1 & 4 \\
\hline pez gato & 3 & 0 & 0 & 0 & 2 & 0 & 0 & 0 & 2 \\
\hline pez martillo & 1 & 0 & 0 & 0 & 0 & 0 & 0 & 0 & $\underline{0}$ \\
\hline pez martillo & 2 & 2 & 1 & 4 & 2 & 0 & 3 & 1 & 13 \\
\hline pez martillo & 3 & 0 & 0 & 0 & 0 & 0 & 0 & 0 & $\underline{0}$ \\
\hline pez rata & 1 & 0 & 0 & 1 & 3 & 0 & 0 & 1 & 5 \\
\hline pez rata & 2 & 5 & 1 & 3 & 7 & 3 & 4 & 9 & 32 \\
\hline pez rata & 3 & 0 & 0 & 2 & 2 & 0 & 1 & 0 & 5 \\
\hline pinguino & 1 & 0 & 0 & 0 & 0 & 0 & 0 & 0 & 0 \\
\hline pinguino & 2 & 0 & 0 & 1 & 0 & 0 & 0 & 0 & 1 \\
\hline pinguino & 3 & 0 & 0 & 0 & 0 & 0 & 0 & 0 & 0 \\
\hline pintadilla & 1 & 0 & 0 & 2 & 1 & 2 & 1 & 1 & 7 \\
\hline pintadilla & 2 & 0 & 1 & 2 & 1 & 3 & 3 & 2 & 12 \\
\hline pintadilla & 3 & 0 & 0 & 1 & 0 & 0 & 2 & 2 & 5 \\
\hline raya & 1 & 9 & 3 & 13 & 23 & 30 & 25 & 25 & 128 \\
\hline
\end{tabular}




\begin{tabular}{|c|c|c|c|c|c|c|c|c|c|}
\hline Species & Phase & Sunday & Monday & Tuesday & Wed. & Thursday & Friday & Saturday & Total \\
\hline raya & 2 & 97 & 42 & 58 & 93 & 105 & 117 & 96 & 608 \\
\hline raya & 3 & 14 & 11 & 21 & 33 & 31 & 33 & 25 & 168 \\
\hline robalo & 1 & 0 & 0 & 0 & 0 & 0 & 0 & 0 & 0 \\
\hline robalo & 2 & 0 & 0 & 0 & 0 & 1 & 0 & 0 & 1 \\
\hline robalo & 3 & 0 & 0 & 0 & 1 & 0 & 0 & 0 & 1 \\
\hline sardina & 1 & 0 & 0 & 0 & 0 & 0 & 0 & 0 & 0 \\
\hline sardina & 2 & 1 & 1 & 1 & 1 & 0 & 0 & 0 & 4 \\
\hline sardina & 3 & 0 & 0 & 0 & 0 & 0 & 0 & 0 & 0 \\
\hline sierra & 1 & 1 & 1 & 0 & 2 & 1 & 1 & 0 & 6 \\
\hline sierra & 2 & 0 & 0 & 0 & 0 & 0 & 0 & 0 & 0 \\
\hline sierra & 3 & 0 & 0 & 0 & 0 & 0 & 0 & 0 & 0 \\
\hline tembladera & 1 & 0 & 0 & 0 & 1 & 2 & 2 & 0 & 5 \\
\hline tembladera & 2 & 1 & 0 & 2 & 2 & 0 & 0 & 1 & 6 \\
\hline tembladera & 3 & 0 & 0 & 2 & 4 & 3 & 5 & 1 & 15 \\
\hline tollo & 1 & 7 & 4 & 17 & 19 & 29 & 32 & 28 & 136 \\
\hline tollo & 2 & 92 & 69 & 50 & 104 & 111 & 115 & 112 & 653 \\
\hline tollo & 3 & 23 & 10 & 15 & 18 & 18 & 31 & 23 & 138 \\
\hline tortuga & 1 & 0 & 0 & 0 & 0 & 0 & 0 & 0 & 0 \\
\hline tortuga & 2 & 0 & 0 & 0 & 0 & 0 & 1 & 1 & 2 \\
\hline tortuga & 3 & 0 & 0 & 0 & 0 & 0 & 0 & 0 & 0 \\
\hline trambollo & 1 & 0 & 0 & 0 & 0 & 0 & 0 & 0 & 0 \\
\hline trambollo & 2 & 0 & 1 & 0 & 0 & 0 & 0 & 0 & 1 \\
\hline trambollo & 3 & 0 & 0 & 0 & 0 & 0 & 0 & 0 & 0 \\
\hline volador & 1 & 0 & 0 & 0 & 0 & 0 & 0 & 0 & 0 \\
\hline volador & 2 & 2 & 2 & 0 & 1 & 0 & 0 & 2 & 7 \\
\hline volador & 3 & 1 & 0 & 0 & 1 & 0 & 0 & 0 & 2 \\
\hline zorro & 1 & 0 & 0 & 1 & 1 & 1 & 1 & 1 & 5 \\
\hline zorro & 2 & 9 & 7 & 1 & 7 & 9 & 9 & 11 & 53 \\
\hline zorro & 3 & 1 & 5 & 6 & 8 & 3 & 8 & 5 & 36 \\
\hline TOTAL & 290 & 784 & 535 & 705 & 980 & 954 & 1050 & 1005 & 6013 \\
\hline
\end{tabular}

Figure 3.2 shows the relative number of trips devoted to boney versus cartilaginous in each of the three phases. La Nina has by and large the most trips associated with it and the learning curves found there tend to dominate the overall distribution in terms of trends in overall effort. 


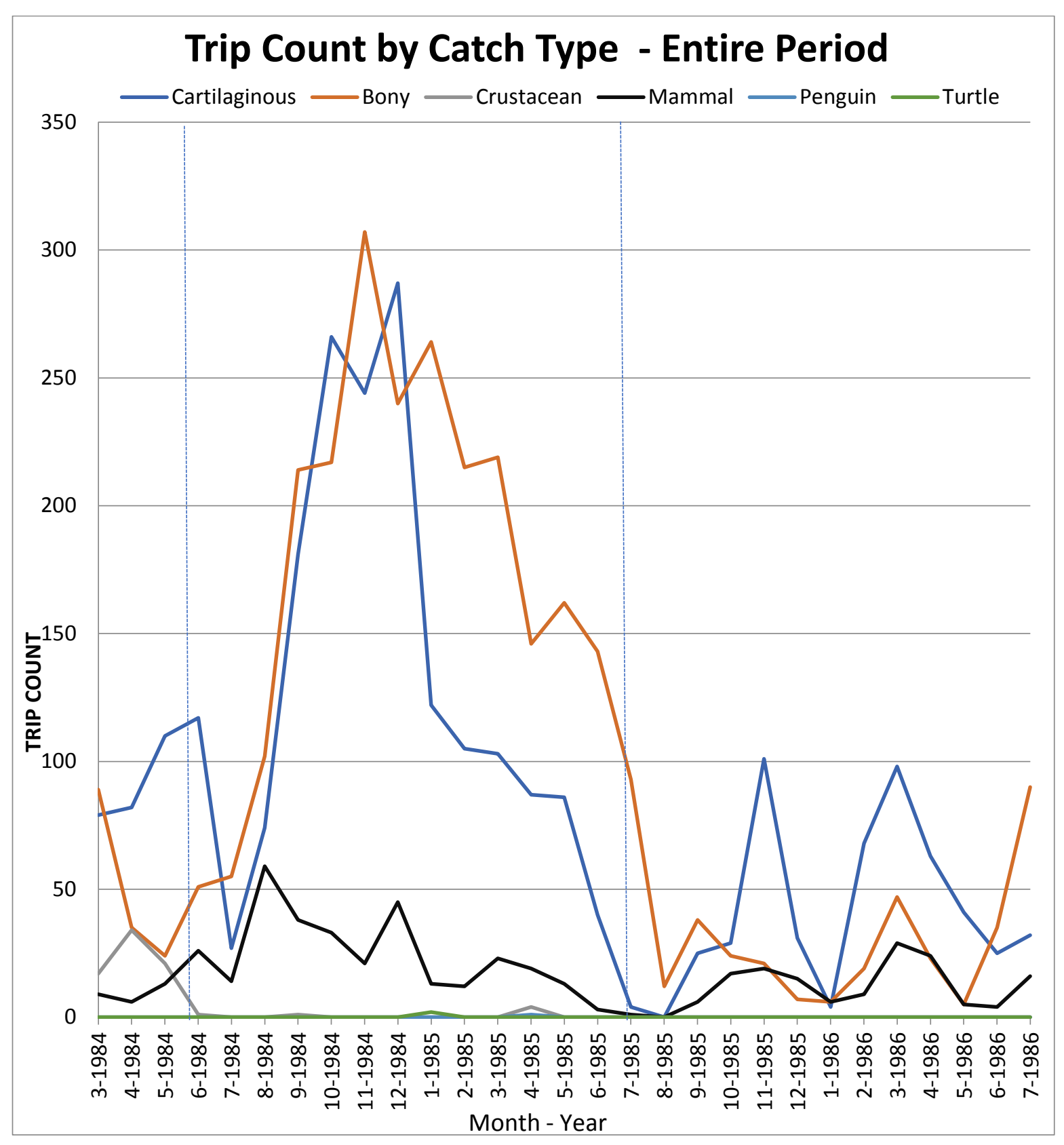

Figure 3.2: The Trip Count by Species Type for the Entire Period

\subsection{CONCLUSIONS}

The results shown in the chapter support the importance of both catch quality on the one hand and the investment of resources in terms of number of trips and over all distance travelled on the other in the description of overall fishing behavior over all three periods. 
Although the patterns can differ between them there are certainly themes that cut across them all. One of the key themes is the learning curve where the number of trips can increase over time during the week relative to certain targeted species. There are indications there is a priority in terms of what catch to pursue first. Another interesting pattern can be seen with fall back catches which mimic trend in targeted catches since as the number of desirable catches starts to dwindle the deficit can be made up by Fall Back categories.

The goals of catch quality and trip investment will be key to the multi-objective model that we develop in Chapter 5. In order to prepare for the computational demands of a multi-objective approach we will employ an extension of the Cultural Algorithm. That will be described in Chapter that follows. 


\section{CHAPTER 4: CULTURAL ALGORITHMS AND MULTI-OBJECTIVE OPTIMIZATION}

\subsection{INTRODUCTION}

Culture, as defined in the American Heritage English Dictionary, is: "The totality of socially transmitted behavior patterns, arts, beliefs, institutions, and all other products of human work and thought" as stated by [21]. Notice that culture composes many different aspects of society, and therefore some part of it is always changing and being continuously updated. Cultural Algorithms (CA) have been used successfully in modeling biological evolution, such as the agriculture in the Valley of Oaxaca, Mexico [2]. CAs have also been applied to concept learning, decision trees, software testing, assessing the quality of a Genetic Program, and data mining, (Engelbrecht, 2007). In this chapter, we will study the Cultural Algorithm in detail and see why it is called a "dual-inheritance" socially motivated problem- solving technique.

We start by an overview of the Cultural Algorithm in sections 4.1 through 4.4. We then follow with a brief history of the development of the Cultural Framework for the solution single objective optimization problems in section 4.5. Section 4.6 describes the version of Cultural Algorithms employed for the Multi-objective problems here. Section 4.7 gives our conclusions.

\subsection{THE CULTURAL ALGORITHM FRAMEWORK}

As we mentioned previously, the Cultural Algorithm is composed of three distinct parts: The Belief Space, the Population Space, and the Communication Protocol based on [2] . Selective information that is learned in the population space can be accepted by the belief space. This updated knowledge becomes part of the decision- making belief space that can be used to influence the next generation in the Population Space. These two parallel flows of 
information, from the belief space to the population space and from the population space to the belief space, are what make Cultural Algorithms a "dual-inheritance" system. Next, we discuss how this information is passed between the two spaces, namely the Communication Protocol. The Communication Protocol contains three main functions for exchanging within the Cultural Algorithm.

First, the Accept function is used to select data from the most-fit individuals to send to the Belief Space. Second, the Update function revises this new accepted information inside the Belief Space. Finally, the Influence function is used to bias the next generation in the Population Space. We can view the Population Space operating at the microevolutionary level, while the Belief Space, changing only after a complete generation operates at a macro-level evolution. The Cultural Algorithm framework is presented in the Figure 4.1, [21]

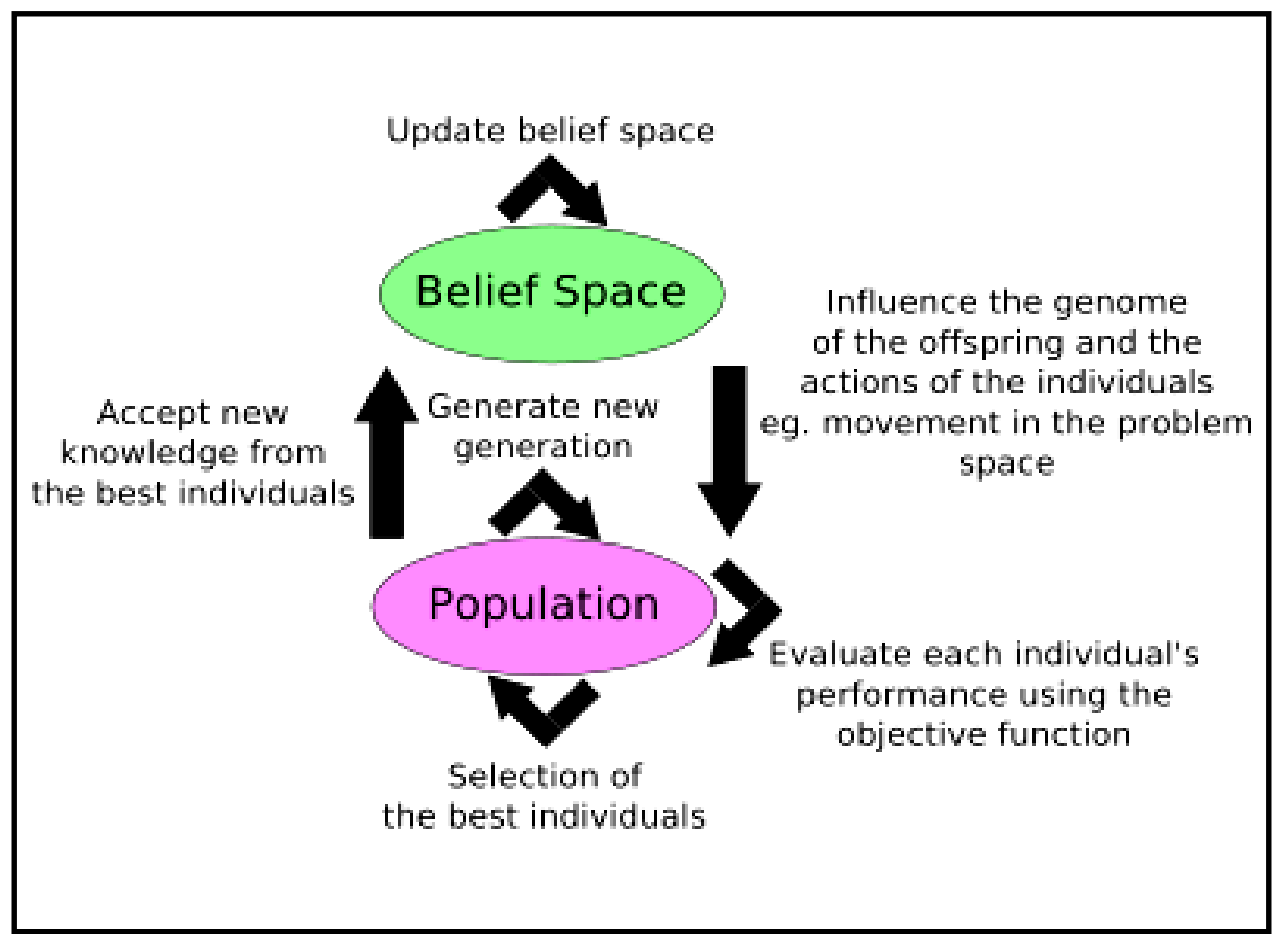

Figure 4.1: The Framework of Cultural Algorithm [21] 
The Population Space can be any standard Evolutionary Algorithm, including Genetic Algorithms, Particle Swarm Optimization, Ant Colony Optimization, or Brainstorm Optimization [22]. We will not discuss the different Evolutionary Algorithms in detail, but rather we will focus on what differentiates the Cultural Algorithm from the others, its Belief Space. The Belief Space allows learned knowledge to be explicitly used to control the problem-solving process. It is therefore a data intensive approach that can support both single and multi-objective problem solving.

We present the pseudo code for Cultural Algorithms in the next figure [14].

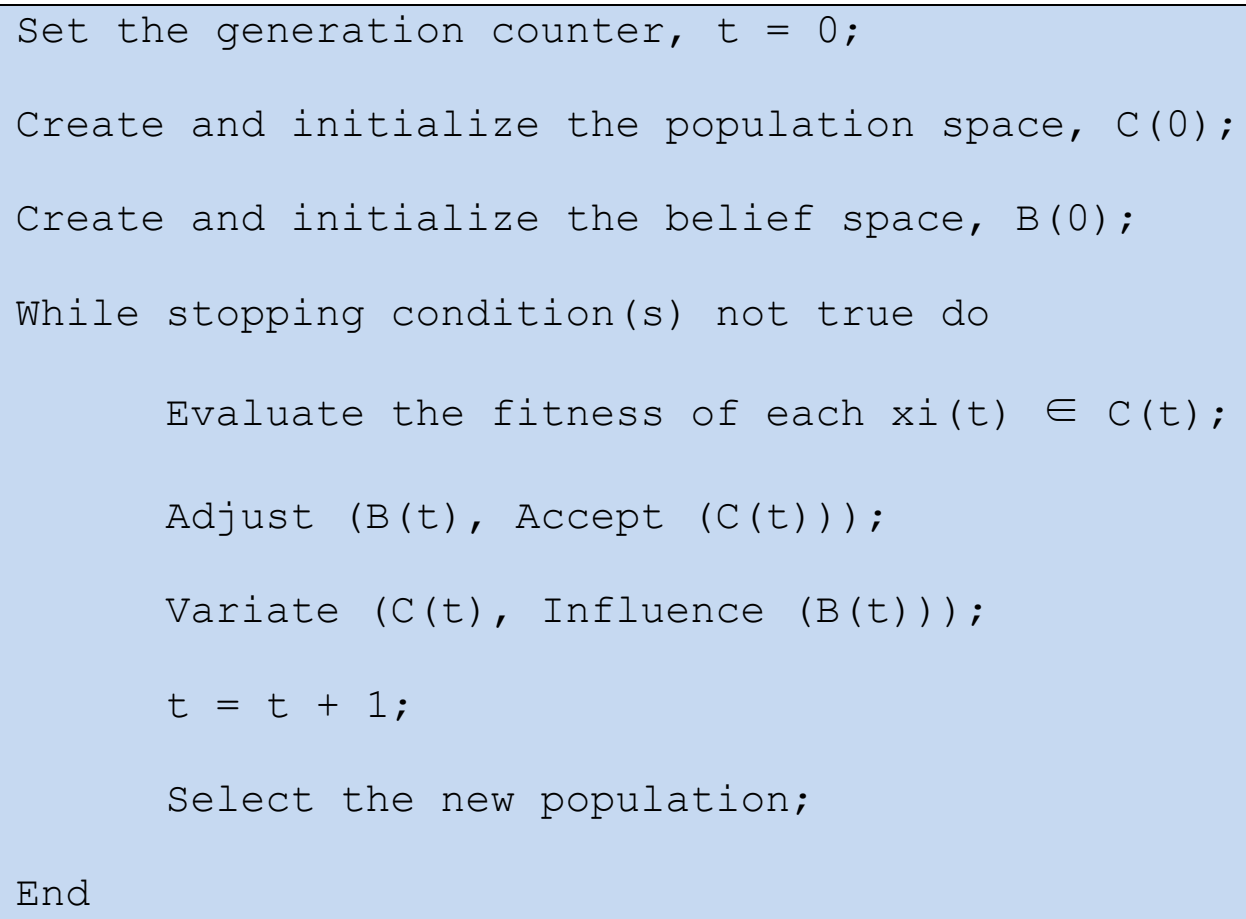

Figure 4.2: Pseudo code for Cultural Algorithms [14]

\subsection{THE BELIEF SPACE}

All five knowledge types were designed to allow evolution-based optimization for a given domain [23]. Each of the knowledge types can provide different levels of performance 
in respect to entire Cultural Algorithm Framework. There is evidence that from Semiotics that each of these formats is used by various species in nature.

Situational Knowledge Source exists to provide an example for others to follow. Usually, the selected elite individuals are used as role-models by non-elites in decision making. The Situational Knowledge Source was incorporated by [24]. Later modifications also accepted sub-elite individuals within a given margin.

Normative Knowledge, also introduced by Chung, uses the Belief Space to store normal or acceptable ranges for a variable's values. Normative Knowledge provides guidelines for individual behavior. In Evolution, the social guidelines are the building blocks of the human social intelligence based on [25]. Normative knowledge is kept current by updating its values to match performance in the current environment.

Topographical Knowledge, "Regional Schema", was added by [27]. The data structure representation is an array of size $\mathrm{n}$ where $\mathrm{n}$ is the number of cells in the mesh. Each cell in the data structure contains a lower and an upper bound for the $\mathrm{n}$ variables indicating the ranges associated with the best solutions found in that cell so far, and a pointer to its children. The topographical knowledge structure is initialized by sampling a solution in every cell in the grid and creating a list of best cells. The update occurs when a cell is divided into sub-cells, when an accepted individual's fitness value is better than the best solution in that cell, or if the fitness value of the cell's best solution has increased after a change event is detected. Topographical Knowledge provides a spatial or array framework in which environmental patterns can be identified and adjusted as needed.

Domain Knowledge was introduced into the Cultural Algorithm system b [28] in order to solve dynamic resource optimization problems. The domain knowledge contains the 
overall system ranges for all parameters and is updated by the most-fit individuals from the population space. Domain knowledge can be used to dynamically predict patterns in the solution landscape. For example, if an upward slope is detected, then we know there will be an increase in resources. The new location of the peak can also be predicted and adjusted simultaneously.

The fifth knowledge type, Historical Knowledge, also referred to as Temporal Knowledge, was added into Cultural Algorithms by [29] to provide global system reasoning and to allow backtracking of actions. It contains information about sequences of environmental changes in terms of shifts in the distance and direction of the optimum in the search space. Information regarding the events and temporal-spatial relations between them is recorded by [30]. Historical knowledge behaves like the system macro-based manager, while Domain Knowledge is more micro-evolutionary.

\subsection{THE POPULATION SPACE}

The acceptance function determines which individuals and their behaviors can impact the Belief Space knowledge. It is often specified as a percentage of the number of current individuals ranging between $1 \%$ and $100 \%$ of the population size and is based upon selected parameters such as performance based on [24]. For example, we can select the best performers, the top ten percent, the worst performers, or any combination. The acceptance function can also be adjusted in a rule-based fashion based upon the state of the problemsolving process. Che utilized the experience of the entire population since the population size was small relative to the number of cones that made up the performance landscape as stated [23]. 
The first influence function was a random integration function, implemented by [30]. From this emerged three phases of problem solving: coarse-grained (explorers) fine-grained (exploitation); and backtracking (retracing). While a given phase is taking place, an individual is likely to be controlled by a knowledge source for several time steps. Thus, an individual is plugged into a role within the population based upon the current state of the problem-solving process.

The five knowledge sources described above were added to Cultural Algorithms at different times, reflecting different problem-solving needs. When used together, they showed interesting collective behaviors regarding different roles in the search process. Since we are applying the Cultural Algorithms to a general set of problem environments it is important that their actions be integrated together. The integration function is maintained by the communication protocol. We will discuss the communication protocol in the next section.

Each knowledge source in the Belief Space holds a different type of knowledge, and each can extract a different experience from groups of individuals. Figure 4.3 summarizes the knowledge update process in the Belief Space. Normative and Topographical knowledge are updated using information about all of the accepted individual experiences in the current generation, which is indicated by orange in the graph. The other three knowledge sources base their updates only on the best performer, which is labeled with magenta. Although each individual knowledge source bases its updates on new knowledge from the current generation of agents, and its own accumulation of knowledge, there is a difference in that some knowledge sources will also use other knowledge sources as part of the update process while others do not. 
In Figure 4.3 we can see that domain and history knowledge use situational knowledge when they are updated. The final difference between the knowledge sources is that updating is called in different situations. Except for History knowledge, all other four knowledge sources are frequently updated by [23] when no environmental dynamics or change in the position of the best value occurs. However, when the environment changes or a new best value is found, History, Situational and Topographical knowledge are updated together.

\subsection{THE COMMUNICATION PROTOCOL}

The communication protocol of a Cultural Algorithm System is composed of two functions: accept and influence [31]. The acceptance function determines which individuals will be selected to update the Belief Space. The influence function determines how the Belief Space guides or biases the population space in generating new solutions. Both functions can use all the five knowledge categories. In addition, we have the update function that coordinates the updating of each individual knowledge source described above, as shown in Figure 4.3 [23]. 


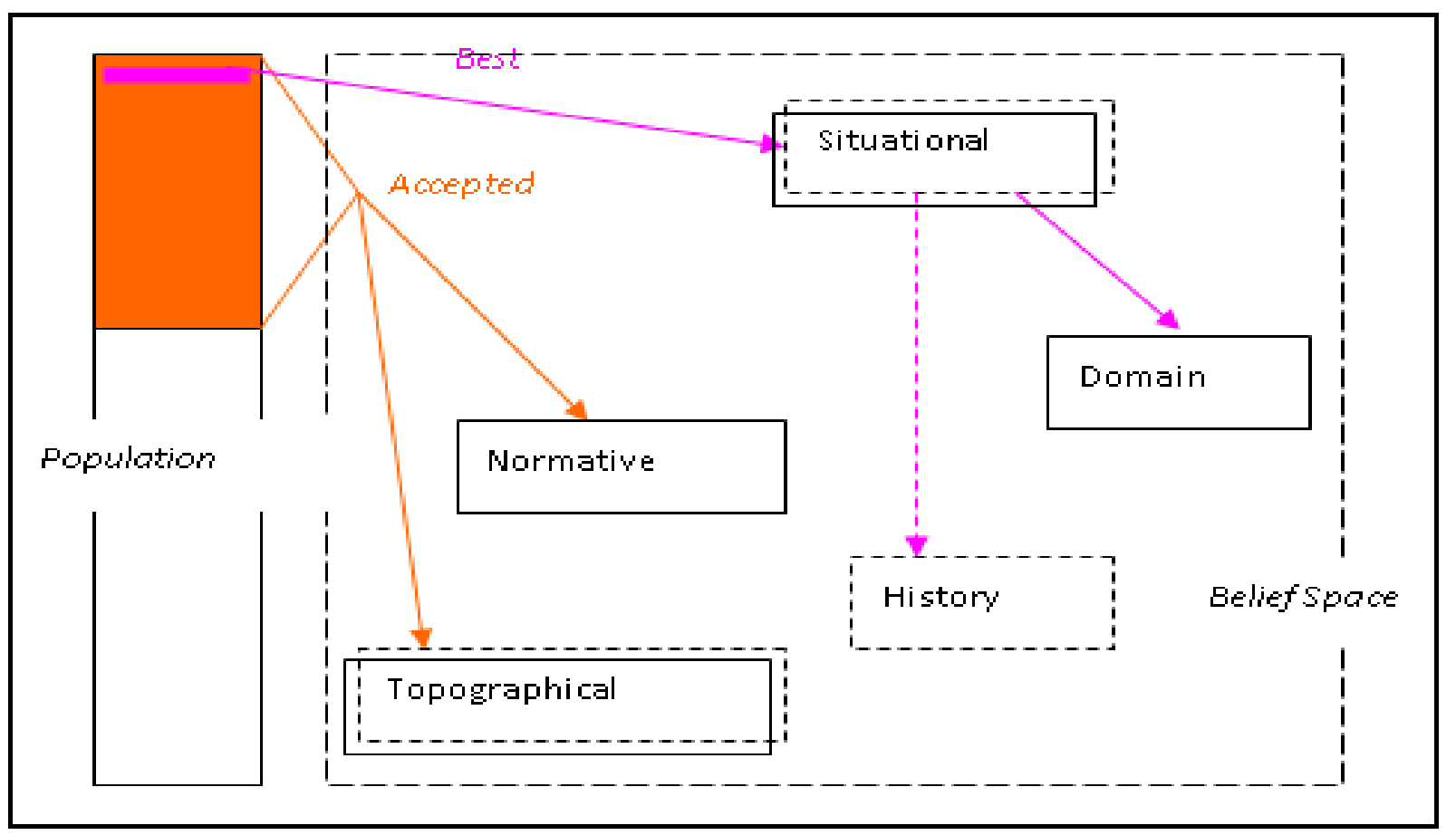

Figure 4.3: Knowledge Update in the Belief Space [23].

\subsection{HISTORICAL DEVELOPMENT OF CULTURAL ALGORITHMS}

In 1979, Reynolds developed the Cultural Algorithm, applying it model the evolution of agriculture in The Valley of Oaxaca, Mexico. His idea was to research motivations of economic and social change in the evolution from a hunter-gatherer to an agriculture society. In these early applications, Reynolds used a theoretical background from Flannery's theory about the origins of agriculture based on Barceló, [31]. Agriculture needs intensive labor resources. This means these labor resources cannot be utilized for other activities, also important for group survival. Reynolds programmed a computer system that calculated the way in which members of this group would arrange their economic activities to approach a balance between investment of agricultural labor and investment of labor in other activities. We provide a history of the Cultural Algorithms and discuss when new components were 
added. We also discuss how the Cultural Algorithm knowledge distribution mechanism changed.

\subsubsection{DEVELOPMENT OF THE FIVE KNOWLEDGE SOURCES}

Chung developed one of the first software systems to implement Cultural Algorithms. His tool kit, Cultural Algorithm with Evolutionary Programming (CAEP), used Normative and Situational Knowledge in the Belief Space and Evolutionary Programming in the Population Space by [24]. Later, Jin added Topographical Knowledge to the Belief Space extending Chung original system by [27]. This key point is this allowed agents to use their regional information when implementing the acceptance, update, and influence function.

Next, Saleem extended a Cones World optimization problem developed by De Jong reacting to the dynamic movement of cones concentration in the environment by [30]. The Cones World problem is to try to find the highest peak in multi-dimensional multi-peak landscape. Saleem needed two additional knowledge sources to implement his new design: history and domain. History Knowledge enabled the system to reason regarding time, while Domain Knowledge provided reasoning with respect to cone attributes: their shape, slope, and altitude. Saleem integration function used random selection of the five different Knowledge Sources, as shown in Figure 4.4 Structure of the Belief Space of CADE (Cultural Algorithm Dynamic Environment by [33].

There was no predetermination as to which knowledge source would influence which individual. The new Cultural Algorithm Framework was named CADE, Cultural Algorithms for Dynamic Environments. Its belief space is shown in the figure below. 


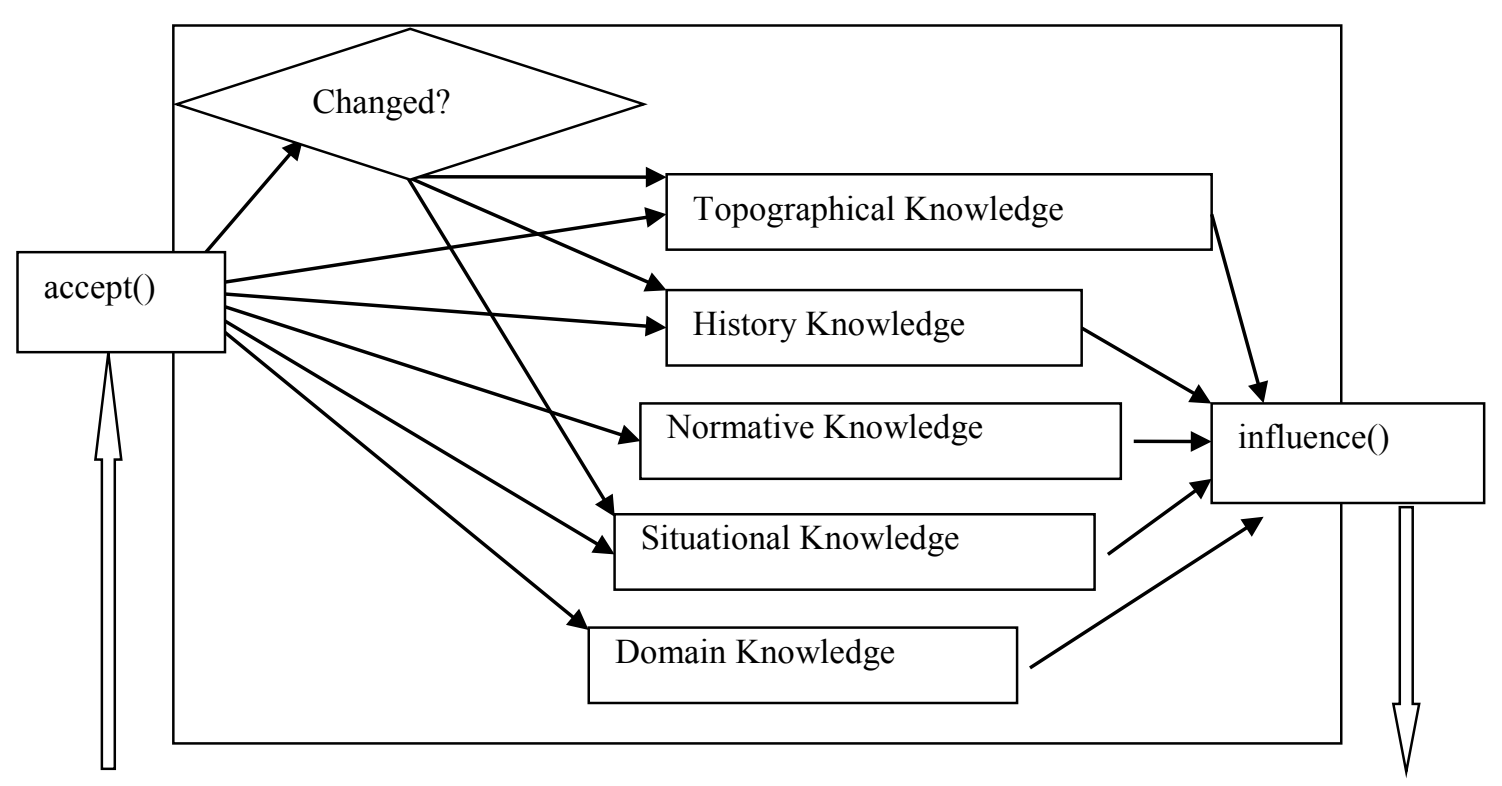

Figure 4.4: Structure of the Belief Space of CADE [33]

\subsubsection{KNOWLEDGE SOURCE INFLUENCE AND THE MARGINAL VALUE}

[31] incorporated an ecological distribution mechanism, namely knowledge swarms from Foraging Theory. She used the Marginal Value Theorem to decide which Knowledge Source will influence which individual. The Marginal Value Theorem (MVT) states that an individual will stay in a patch of land until the current resource gain is less than the average expected value for the population. From basic animal instincts, the individual will keep moving to another patch that satisfies the marginal value constraint by [31]. The size of a knowledge sources area under the wheel is a function of its ability to produce above average gain as stated by [31]. The better the performance the larger the pie slice on the wheel. Each of the five knowledge sources is initially given $20 \%$ of the wheel area. In Figure 4.5 , the left represents the initial evenly allocated sections. The pie chart on the right shows how some knowledge sources began outperforming the others. 


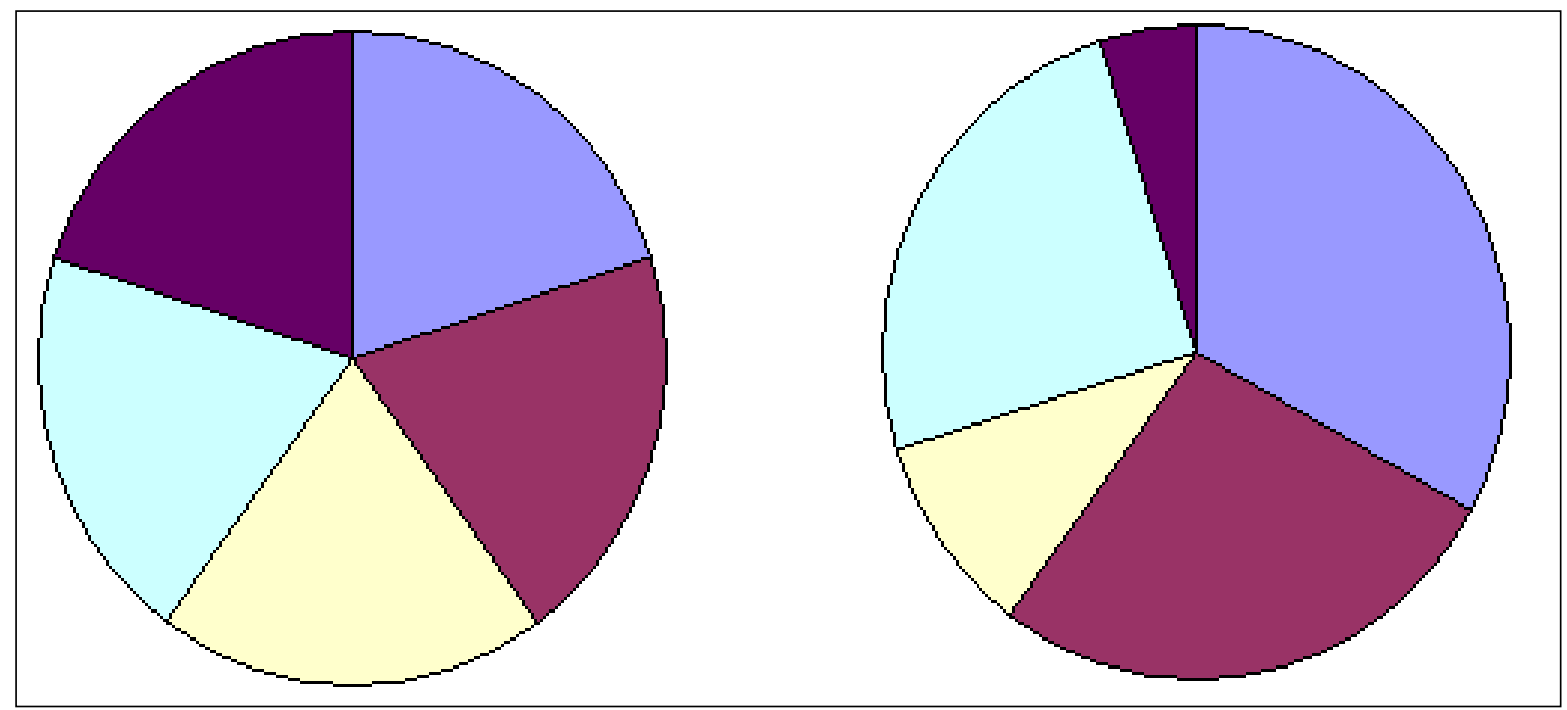

Figure 4.5: Example of the Roulette Wheel Function [31]

Peng referred to the Knowledge Sources as predators, competing to gain control over the individuals, the prey, in the population space.

\subsubsection{THE SOCIAL FABRIC AND MAJORITY WINS}

The Social Fabric, introduced by [21], now allowed an individual to look at what their "neighbors were doing", instead of forcing each agent to act independently. The network topology type, such as square, 1-best (local), or g-best (global) was kept constant, but the positions of the agents within the network were randomly selected at each time step. In this way, Ali was able to accurately study the impact of just adding in the communication links to the search process. Since the connections were dynamically re-established at each time step, Ali viewed this as a weaving process and thus coined the term, the Social Fabric introduced by [21].

Ali wanted to investigate what social structures emerge in the population when knowledge sources can influence individuals through a social network. Ali embedded the existing Cultural Algorithms framework into the Repast Toolkit and named his new system, 
Cultural Algorithms Simulation Toolkit (CAT) by [21]. It represents the extent to which the influence of a knowledge source can spread through a population. The interconnections between agents in the population were viewed metaphorically as a social fabric, created by the interactions between agents. Knowledge sources in the Belief Space select individual from the population to influence individual agents in the population. In Figure 4.6 we present Schema of Social Fabric developed by [21]. Each knowledge source in the Belief Space is color coded and its color is applied to the individual agent in a network that it is to influence at that time step. The network shown in the diagram is a homogeneous square network; everyone has exactly four neighbors. Each of those agents has a position on the landscape. Ali used three homogeneous networks in his version: lbest (degree of two for each agent), square (degree of 4 for each agent), and gbest (degree of $n-1$ nodes for each agent). lbest and global topologies are shown in Figure 4.7. 


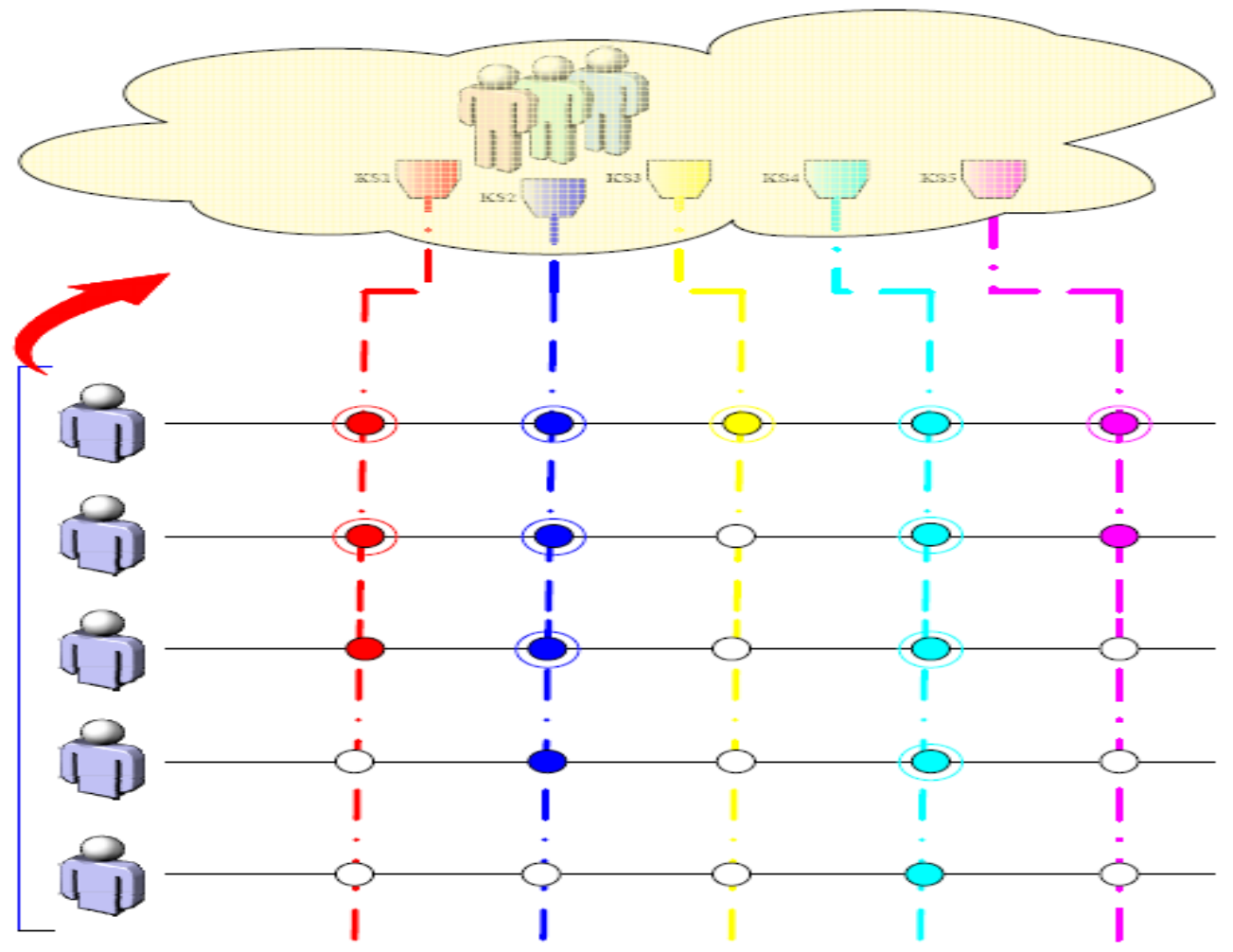

Figure 4.6: Schema of Social Fabric [21]

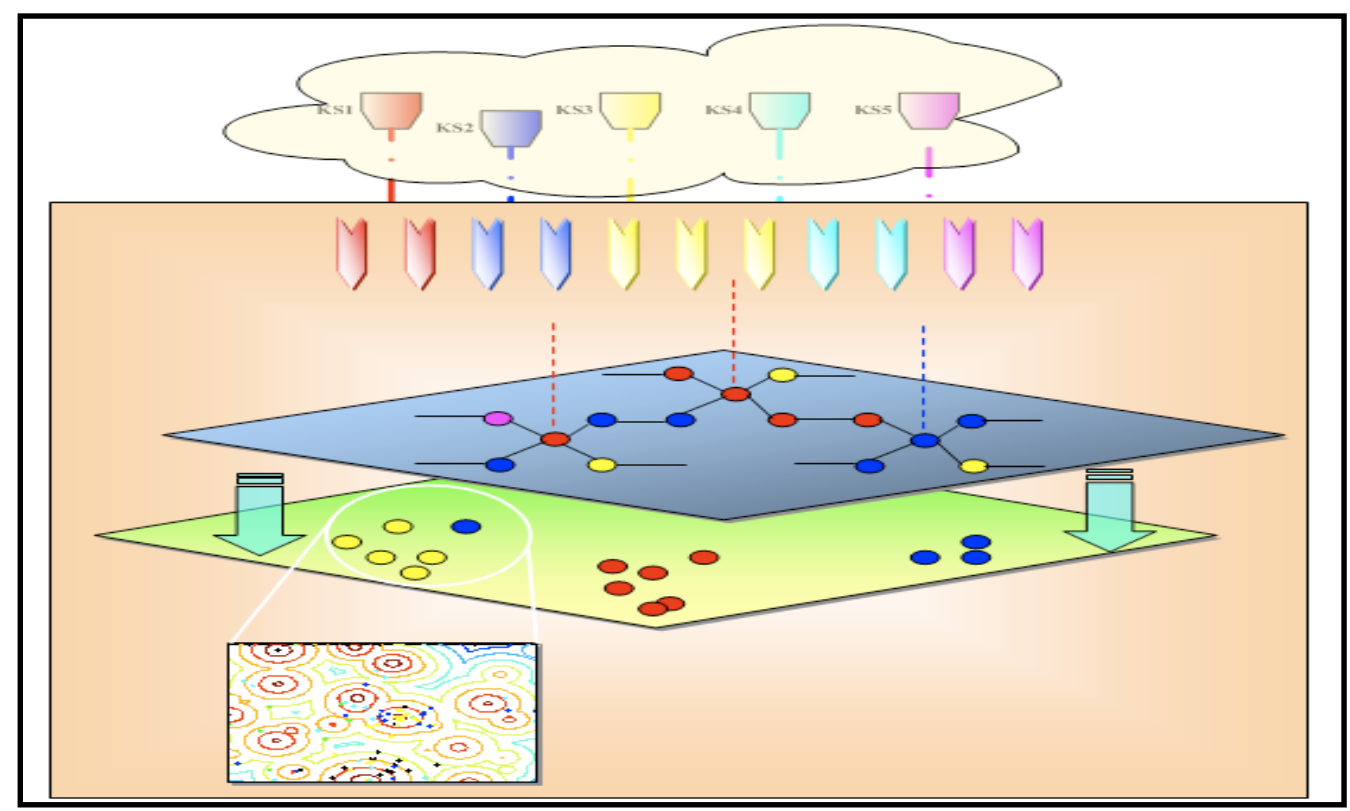

Figure 4.7: The Social Fabric component in CAT 1.0 [21] 


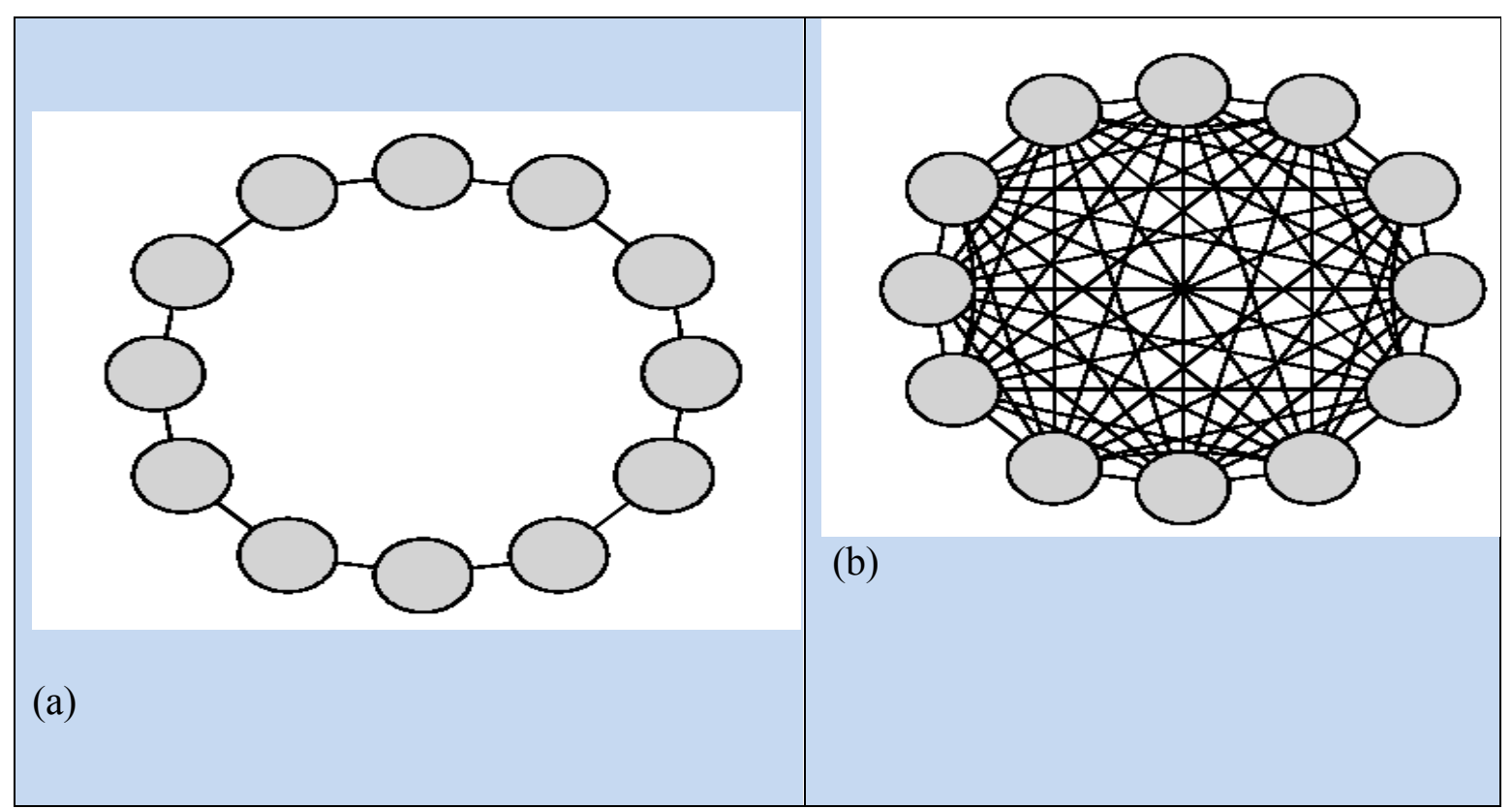

Figure 4.8: Topologies in the Social Fabric [21]

In Figure 4.8 we see the Topologies in the Social Fabric (a) lbest topology. (b) gbest topology, as presented by [21]. The network organization established at the beginning of the run and the connections between individual agents remained constant over the course of the problem-solving process even though the agents were able to move to different positions on the landscape, thus a homogenous and not heterogeneous network. This fixed network gives a type residual memory.

At each time step, every individual is influenced by one of the knowledge sources. Knowledge Sources do not know anything about the network and the selected individuals' position in it and vice versa. The process is a double blind. The individual then transmits the name of the influencing Knowledge Source to its neighbors through as many hops as specified. Next, each node counts up the number of Knowledge source bids that it collects. It will have the direct influence from the Knowledge Source that selected it, plus the ID's of the 
Knowledge Sources transmitted to it by its neighbors. The Knowledge Source that has the most votes is the winner and will direct the individual for that time step.

\subsubsection{NICHING AND WEIGHTED MAJORITY WINS}

Che extended Ali's original CAT 1.0 system by adding a homogenous social network structure developed by [23]. Che experimented with how knowledge sources can influence individuals using different kinds of social networks. He also removed randomization in the social fabric and node assignment by assigning a unique id to everyone. Each generation remembers its previous neighbors. The neighborhood is homogenous and does not change throughout the entire simulation as stated by [23]. This allows everyone, regardless of the current position in their environment, to utilize information from the knowledge source of its neighbors to make a better-informed decision. To implement this, Che added two components to the existing influence function: (1) utilizing the knowledge sources of individuals and the transmission to immediate neighbors used a fixed topology and (2) the selection of the knowledge source for everyone determined by using its own influence combined with their neighbors, an "incentive-based decision-making function" as introduced by [23]. He called the modified CAT system, CAT 2.0.

[23] modified the decision-making approach to allow for an incentive-based scheme. Each of the votes received by an individual is associated with a weight. The selected Knowledge Source is the one with the most total weight. This supported the co-evolutionary focus since less frequently used knowledge sources would produce a new result, exceeding the Knowledge Source that is used most frequently stated by [23] .

The modification is based upon a fundamental voting technique used in the earliest Cultural Algorithms and again reflecting the predator-prey approach to co-evolution. 
Incentive based Majority Win developed by [31] when everyone calls the influence function, the influence function will have a "direct" knowledge source designated to this individual by spinning the knowledge wheel. At the same time, this individual can also receive information from its neighbors regarding controlling knowledge sources. In Figure 4.9, we present an example of individual with Octagon Network topology. Agent A0 is directly influenced by S (Situational KS) and there are an additional three neighbors that are currently influenced by S. Using Majority Wins, the Situational Knowledge Source, with a total count of four, wins the right to influence the current individual, A0. Figure 4.10 shows the majority win process.

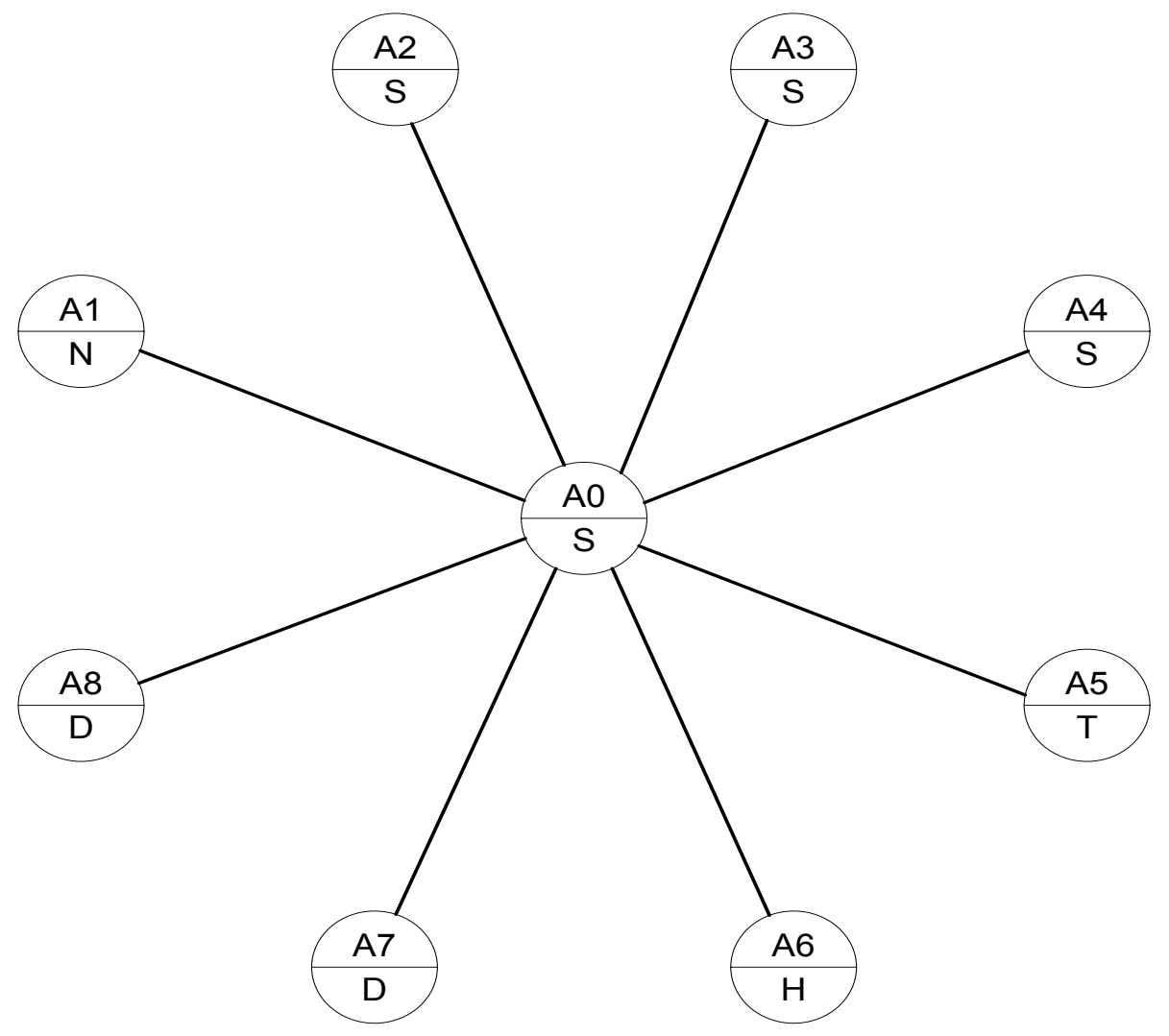

Figure 4.9: Knowledge Source Interaction by [23]. 


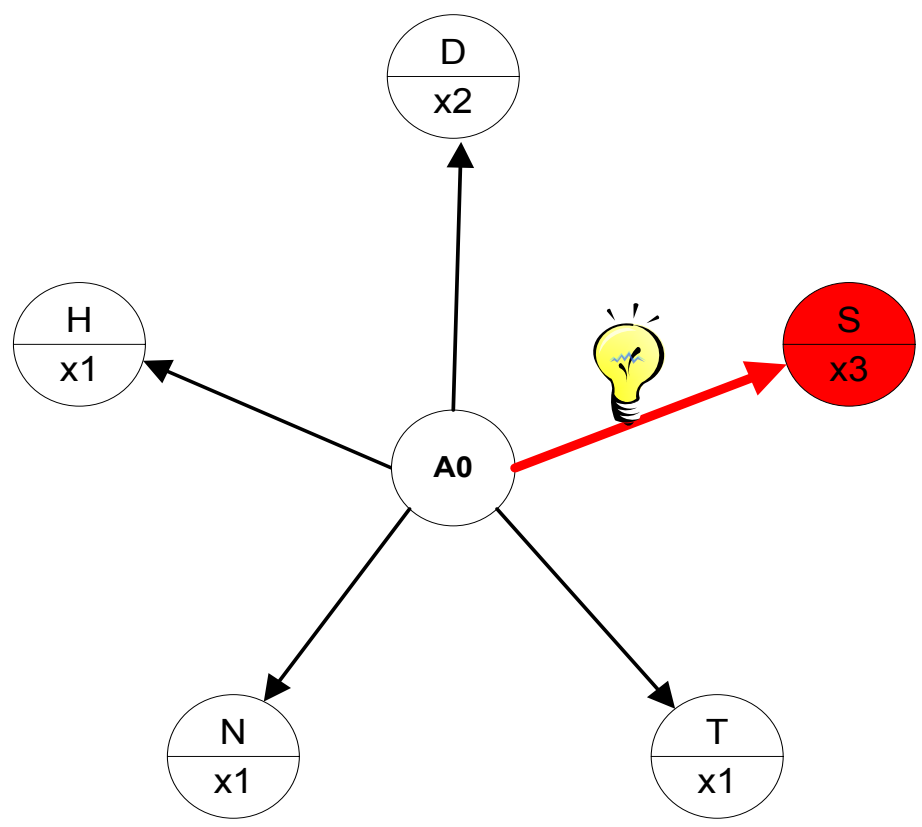

Figure 4.10: Majority Win in Belief Space by [23].

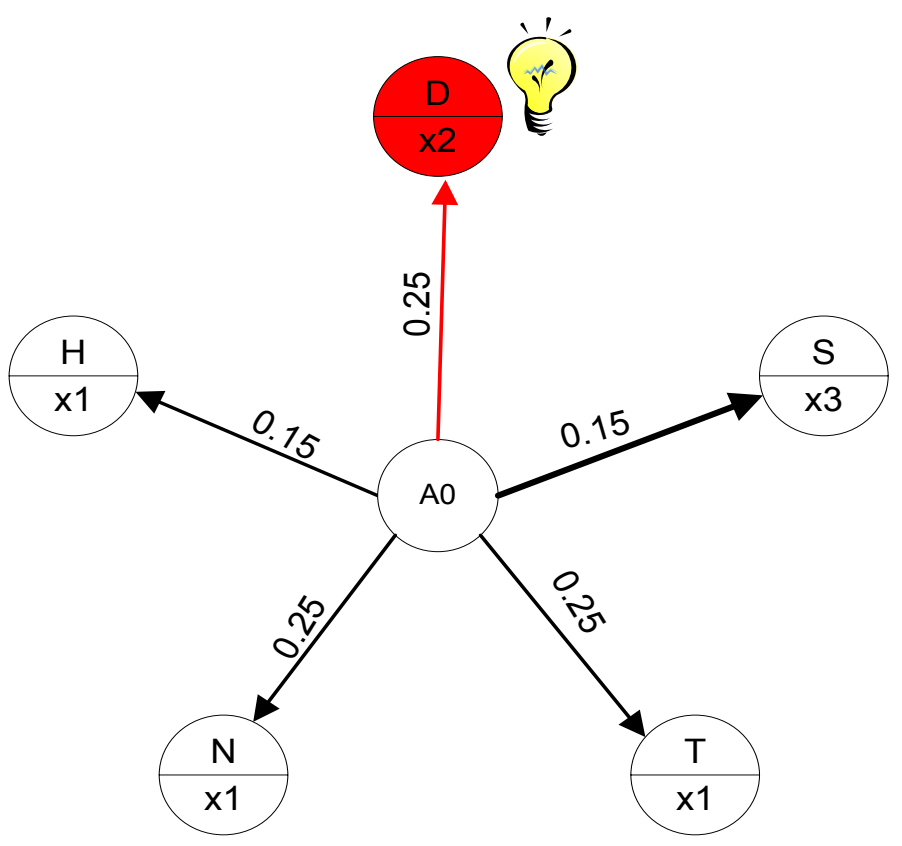

Figure 4.11: Weighted-Majority Win in Belief Space by [23].

Reynolds introduced vector voting as a decision-making model for hunter-gatherer society simulation. Che's addition used the current average fitness of each Knowledge 
Sources as weight of each Knowledge Sources count then applying a majority win based on the weighted count as shown in Figure 4.11. In Figure 4.10, Knowledge source S wins, but in Figure 4.10, D wins because of the weighting; $.25 * 2=.5$ whereas $\mathrm{S}$ has $.15 * 3=.45$. All the previous voting mechanisms do not allow more than one Knowledge Source to influence an individual. What if more than one Knowledge Source could cooperate with another, would this improve the overall performance of the Cultural Algorithm? One type of framework that models cooperation or competition in the Knowledge Distribution Mechanism is shown in Figure 4.12.

One of the designs of the network topology of the CA is to allow communication between individuals by implementing a simple memory of past actions as stated [21] . A wide range of systems in nature and society can be described in terms of complex networks. Examples include the Internet, a network of interconnected routers, or human social systems [21]. With recent advances in modeling such complex systems it is apparent that the topology and evolution of real networks are determined by robust organizing principles. We are interested in the various topologies that have been used and have become implanted within the mathematical frameworks and graph theory, [21]. To really understand the observed effects in the system it is necessary to include additional components of the social network to the model. For example, in a social system one can examine the effects of knowledge flow between a chain of individuals across the network represented by a certain topography which can be static or dynamic. [21] stated that, Multi-layered networks are a beneficial addition to the study of agent-based simulations. 


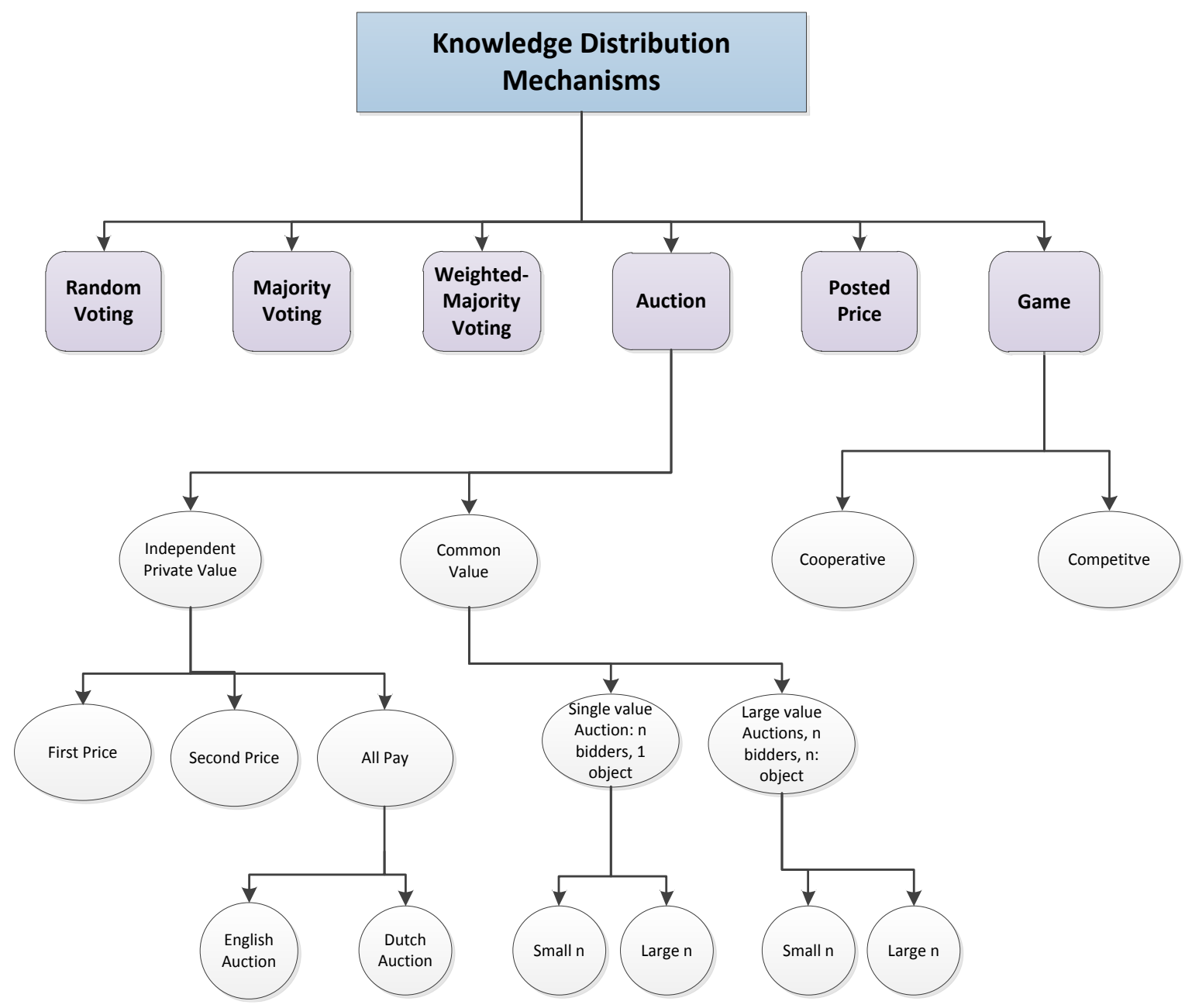

Figure 4.12: Different Knowledge Distribution Mechanisms.

Additional network layers describing the different sets of relationships can be included to allow us to describe to what extent everyone can affect and interact with other individuals on the test-bed landscape. In addition, the rise of cultural influences can also add to the dynamics of the network and contribute in the formation of a network. Multi-layered networks have been used in the modeling of past societies using Cultural Algorithms [21]. For example, the collapse of the Ancient Anasazi in south-western Colorado can be modeled as allowed a hierarchical collection of networks which evolve over time within the context of Cultural Algorithms developed by [6]. 


\subsection{THE MULTI-OBJECTIVE MODEL}

Single objective optimization problems are suitable for situations in which the different sub-problems that contribute to the performance function are correlated with each other. However, often in the real-world objectives are conflicting in nature; the achievement of one performance goal can be at the expense of another. Problems like this are called multiobjective problems where each goal is associated with its own objective function. The goal is to allocate resources in such a way that each receives a certain level of performance. Both Best and Liu have used Cultural Algorithms in the past to solve multi-objective benchmark problems [15]; [33] . In this thesis we will employ a version of Cultural Algorithms to solve a real-world multi-objective problem, the artisanal fishing problem. While only a brief overview is presented here the approach is described in more detail in [35].

A multi-objective problem can be formally described in terms of the set of functions to be optimized, the set of constraint functions, and the parameters along with parameter ranges. A general formulation of a multi-objective problem can be written as such:

Let $F:\left\{f_{1}, f_{2}, f_{3}, \ldots, f_{n}\right\}$ be the set of objective functions.

Let $G:\left\{g_{1}, g_{2}, g_{3}, \ldots, g_{m}\right\}$ be the set of constraint functions.

Let $\vec{x}=\left\langle x_{1}, x_{2}, x_{3}, \ldots, x_{k}\right\rangle$ be the vector containing the parameters.

Let $\left[r_{i_{1}}, r_{i_{2}}\right]$ be the range for each parameter $x_{i}$.

In a multi-objective problem, "optimizing" the objective function set may involve maximizing some functions while minimizing some others.

As described previously a Cultural Algorithm must have a Population Space, a Communication Protocol, and a Belief Space. For the CA developed for this application we employ a particle swarm population model. Each particle represents a vector and it can be 
controlled by one or more knowledge sources in the Belief Space. It can be interpreted is a version of the traditional Particle Swarm Optimization (PSO) algorithm developed by [36]. The acronym for the system is thus CAPSO (Cultural Algorithm Particle Swarm Optimization. The vanilla Particle Swarm algorithm is given below. There, each individual is influenced by the value of its best performing neighbors (GBEST).

1. Initialize an array of particles with random positions and velocities on $D$ dimensions,

2. Evaluate the desired minimization function in D variables,

3. Compare evaluation with particle's previous best value (PBEST[]): If current value $<$ PBEST[] then PBEST[] = current value and PBESTX [][d] = current position in Ddimensional hyperspace,

4. Compare evaluation with group's previous best (PBEST[GBEST]): If current value $<P B E S T[G B E S T]$ then GBEST=particle's array index,

5. Change velocity by the following formula:

$$
\begin{aligned}
& V[][d]=V[][d]+ \\
& A C C-C O N S T * \text { rand }) *(P B E S T x[][d]-\text { Present X }[][d]) \\
& + \\
& A C C-C O N S T * \text { rand }) *(P B E S T x[G B E S T][d]-\text { Present } X[l[d]), \text { and, }
\end{aligned}
$$

6. Move to Present $X[][d]+v[][d]:$ Loop to step 2 and repeat until a criterion is met.

However, in our variant, particle velocities are controlled by the knowledge sources in the Belief in PSO but through a VEGA-like [37] mechanism. We provide pseudocode below for CAPSO's CA variant [35].

CA.Initialize() // Initialize the Belief Space

CA.CreateSituationalKnowInitGuesses(numInitGuesses)

\section{//The Population Space}

objfuncs $=$ [objective functions provided by problem/user $]$ pop $=$ Population.Initialize(locations, velocities) pFront $=$ ParetoFront.Initialize () 
do repeat until termination condition:

Foreach indiv in Pop:

indiv.position $+=$ indiv.velocity

Foreach indiv in Pop:

If no pFront members dominate or equal $\mathrm{F}$ (indiv):

pFront.Add(F(indiv))

If $\mathrm{F}$ (indiv) dominates an item( $\mathrm{s}$ ) in pFront:

remove dominated item(s) from $\mathrm{pFront}$

//VEGA method of selecting elites

elite $=[]$

foreach objf in objfuncs:

elite.Add(Pop.Select(top 1/7 of performers according to objf)

elite.Remove(any duplicates)

//GA Method of evolving particle velocities

foreach indiv in pop and not in Elite:

Rnd $0=$ random.between $(0,1)$

Rnd $1=$ random. between $(0,1)$

Rnd2 $=$ random.between $($ Rnd 1,1$)$

Rnd3 = random.between $($ Rnd2, 1)

Rnd4 $=$ random.between $($ Rnd3, 1)

If rnd $0<$ rnd1: //both crossover and mutation

Indiv.velocity $=$ Crossover(elite.pickrandom().velocity, indiv.velocity)

Indiv.velocity $=$ Mutation(Indiv.velocity)

Else if rnd0 < rnd2: //(crossover but no mutation)

Indiv.velocity $=$ Crossover(elite.pickrandom().velocity, indiv.velocity)

Else if rnd0 < rnd3: //(mutation but no crossover)

Indiv.velocity $=$ Mutation(Indiv.velocity)

Else if rnd $0<\operatorname{rnd} 4 / /$ (weighted average between part's velocity \& an elite's)

Indiv.velocity $=$ vectorWgtAvg(elite.pickrandom().velocity, indiv.velocity)

Else: \#Neither crossover nor mutation

//END PSO Population

CA.Acceptance(elite)

CA.Update() 
Foreach indiv in pop but not in elite:

$\mathrm{ks}=\mathrm{CA}$.ChooseKnowledgeSource(sit, norm, or hist)

targVelocity $=$ CA.Influence(indiv, $\mathrm{ks}$ )

indiv.velocity $=$ vectWgtAvg(indiv.velocity, targVelocity)

indiv.knowSource $=\mathrm{ks}$

The Cultural Algorithm - Particle Swarm (CAPSO) configuration used here employs situational knowledge to store the non-dominated vectors that constitutes the Pareto Front under construction. In addition it implements the topographic knowledge function as a multithreaded, multicore-capable "drill-down" mechanism that "spawns" a number of competing particle swarms. These take the place of the regional schema associated with Topographic knowledge discussed earlier. It assigns each of them to a separate child thread connected to a single knowledge source which is a shared structure located on the main thread to accept and distribute knowledge from each of these child threads. Below is pseudocode for CAPSO's multithreaded drill-down mechanism:

subspaceDims $=$ the set of dimension lengths of each subspace that the search space //will be divided into. This is given as an argument to the program/

subspaces $=$ divide searchSpace into equal portions of subspaceDims dimension lengths:

//For each subspace within the search space spawn a new thread, spawn a new particle //swarm in that portion of subspace, then attach the particle swarm to the thread.

foreach subspace in searchSpace: newThread $=$ new Thread(new particleSwarm(subspace) $)$ threads.add(newThread)

while(threads.any.isActive()):

//wait for all the threads to come in

//After all the threads have come in, reports about what happened in the program $/ /($ learning curve data, knowledge source dominance data, etc.), are printed. Reports.Print() 
After each run of the population individuals are selected to contribute to the Belief Space. They are used to update the scores for the competing knowledge sources. These Knowledge sources are invoked stochastically on the basis of point totals acquired from the control of particles in the population.

1. If a solution set (represented by a point in vector-space) is added to the Pareto Front and (but) does not dominate any existing point on the currently constructed Pareto Front, 5 points are added to the total score for the knowledge source currently influencing that particle.

2. If it is the initial vector added to the Pareto Front then the associated knowledge source is given 10 points for initiative.

3. If the new point does dominate one or more existing points within the Pareto Front, then the absolute value of the vector distance between the new point and the closest dominated point is added to the score for the associated knowledge source.

Knowledge sources are then selected to influence mini-swarms based upon their scores for the current generation.

\subsection{CONCLUSIONS}

In this chapter the basic Cultural Algorithm was first described. We then provided a history of its development. The Chapter concluded with a description of a variation of the Cultural Algorithm that used a PSO based population for the specific solution of multiobjective optimization problems such as the one described here, CAPSO. With Stanley's design of CAPSO, the implementation was carried out as part of collaborative work by [35].

This configuration will be used to statistically test the differences in the spread of different tours between the three ENSO phases in Chapter 7. In other words, while there 
appear to be differences in the shape of the generated Pareto curves developed in Chapter 6 via our simulation model, are those statistically different from each other and from other linear models? This is a computationally intensive task for which the Cultural Algorithm with its intensive use of knowledge to drive the search will be good at. 


\section{CHAPTER 5: ARTISANAL FISHING MODEL}

\subsection{INTRODUCTION}

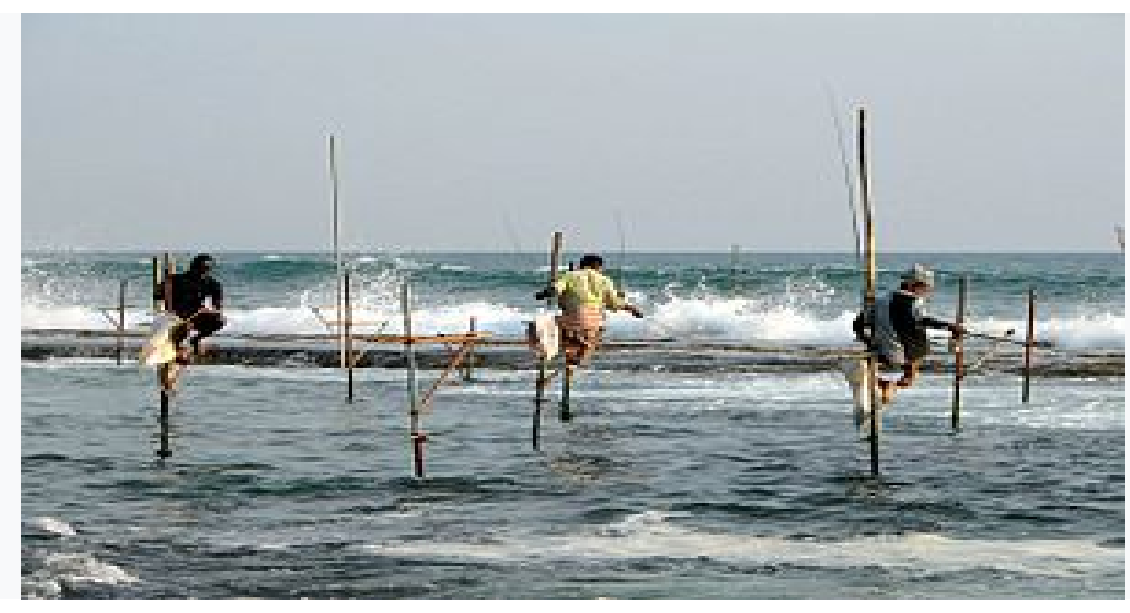

Figure 5.1: Artisanal fisherman - traditional techniques [37]

Artisanal Fishing agents employ small-scale, low technology, and low capital fishing practices conducted by non-commercial agents. The techniques can be in the form of cast nets, harpoons, arrows, rod and reel, and small boats. These agents are often defined as part of a familial unit. They will often make short overnight fishing expeditions close to the shore. Their catches are not often exported and more likely to be consumed locally. However, the Payout from the trips can be sold commercially and used for subsistence. What differentiates it from large-scale industrial fishing is that it is less stressful on the environment than commercial fishing can be.

The data produced by Marcus described artisanal fishing practices over a period of two and one-half years centered at the modern site of Cerro Azul. During the time the environment was affected by a large ENSO activity. This provided us with the opportunity to observe the decision-making adaptation made by the fishermen to these changes over time. Since this was not the first ENSO that fishermen were exposed to in the area, we assume that 
the community response to the environment perturbations reflected collective practices that have emerged over time.

Our initial analysis discussed in Chapter 3 suggested that the patterns of fishing behavior observed in the dataset reflected two basic principles:

1. Catch desirability played a role in the scheduling of fishing trips in all three of the phases to different degrees.

2. The resources required to support a successful fishing trip differed in magnitude from one phase to the next. For example, in order to support a successful trip in La Nina it required more of an investment in resources such as gas than in other phases.

These two goals can be viewed as conflicting in the sense that the resources needed to generate just any catch may be a lot less than those needed to produce a highly desired catch. In other words, goal 2 just reflects the opportunity to produce a successful trip whereas goal 1 suggests that a trip a commercially desirable catch is most important.

From a broader perspective goal \#1 reflects the need for profitability with regards to the artisanal fishing activity for a given household. If given the opportunity to choose between a catch that can fetch a higher local market value than another, this goal would be in favor of targeting that catch. To the extent that this can be done over a succession of trips for a family, the presumed social unit here, the fishing agent can even reap a profit over time.

While goal \#1 stresses profitability, goal \#2 relates broadly to the issue of sustainability. That is, the agent needs to invest sufficient resources into a trip in order to bring back something in order to sustain the family unit and perpetuate the fishing activity. It reflects the general goal of just being able to get out and fish on a given day. 
The two goals constitute part of a bi-objective optimization problem. The solution to such a problem can be viewed in terms of what is called a Pareto front. A Pareto front is a set of Pareto efficient allocations of resources to goals such that it is impossible to reallocate one in order to make it better off without making the other criterion or goal worse off. It was named after an Italian engineer and economist and has been applied to many situations such as game theory. A Pareto efficient allocation is said to be Pareto optimal. A Pareto front is a collection of Pareto optimal points.

Figure 5.2 gives an example of a Pareto curve. Points on the curve are said to be nondominated in the sense that an increase in one goal at that point will cause a corresponding decrease in performance for the other. In the Figure points A and B are said to dominate C since at point $\mathrm{C}$ an improvement in $\mathrm{f} 1$ can also not necessarily decrease the performance of $\mathrm{f} 2$ around the that point as illustrated by both A and B.

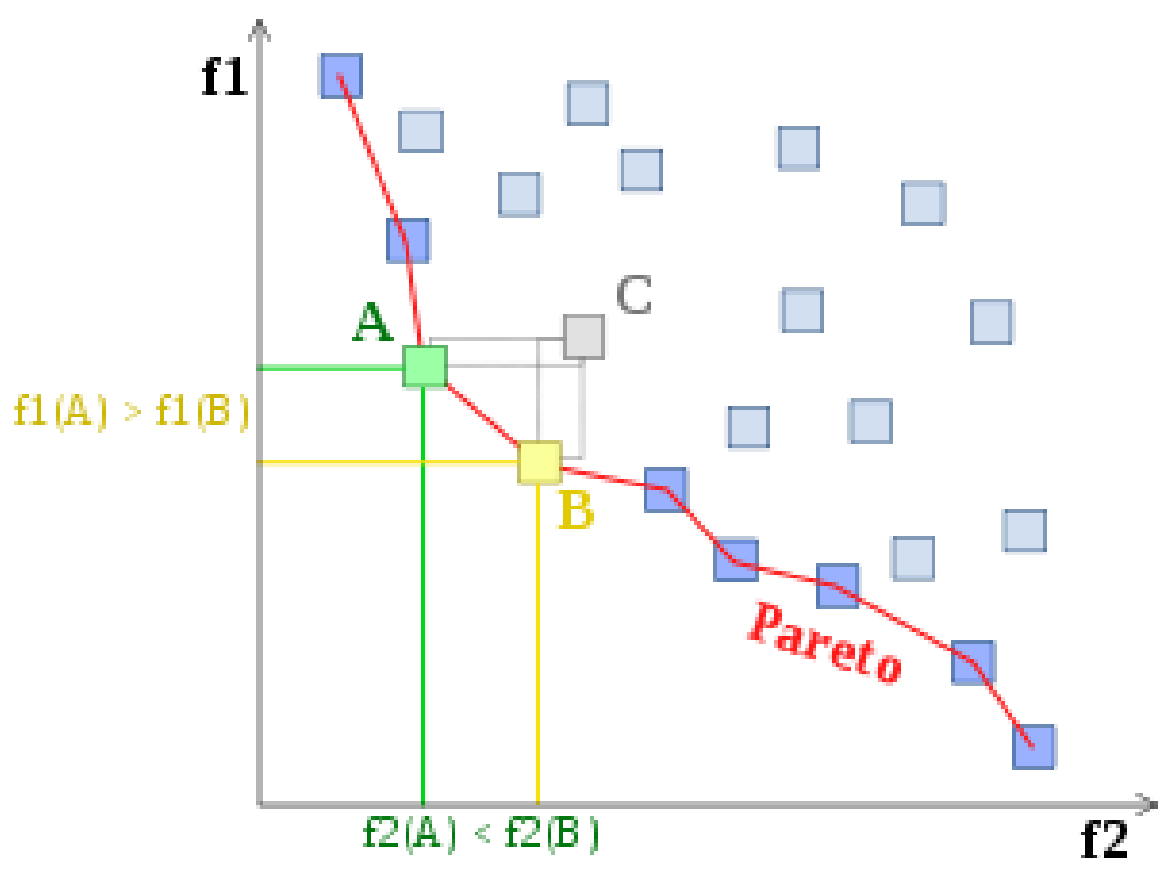

Figure 5.2: The Pareto curve is the set of non-dominated points [38] 
Our goal will be to produce a general model for the characterization of fishing behavior in terms of these two fundamental goals and apply them to the simulation of fishing strategies here. The result of these applications will be to produce a Pareto front that is indicative of the tradeoffs that need to be made by local fisherman in responses to environmental changes during the period of observation. Figure 5.3 gives an example of the resultant Pareto Frontier for one of the strategic scenarios that we will be testing the model in. (Effort for trips taken over a Full Week in Phase 1, the Residual El Nino)

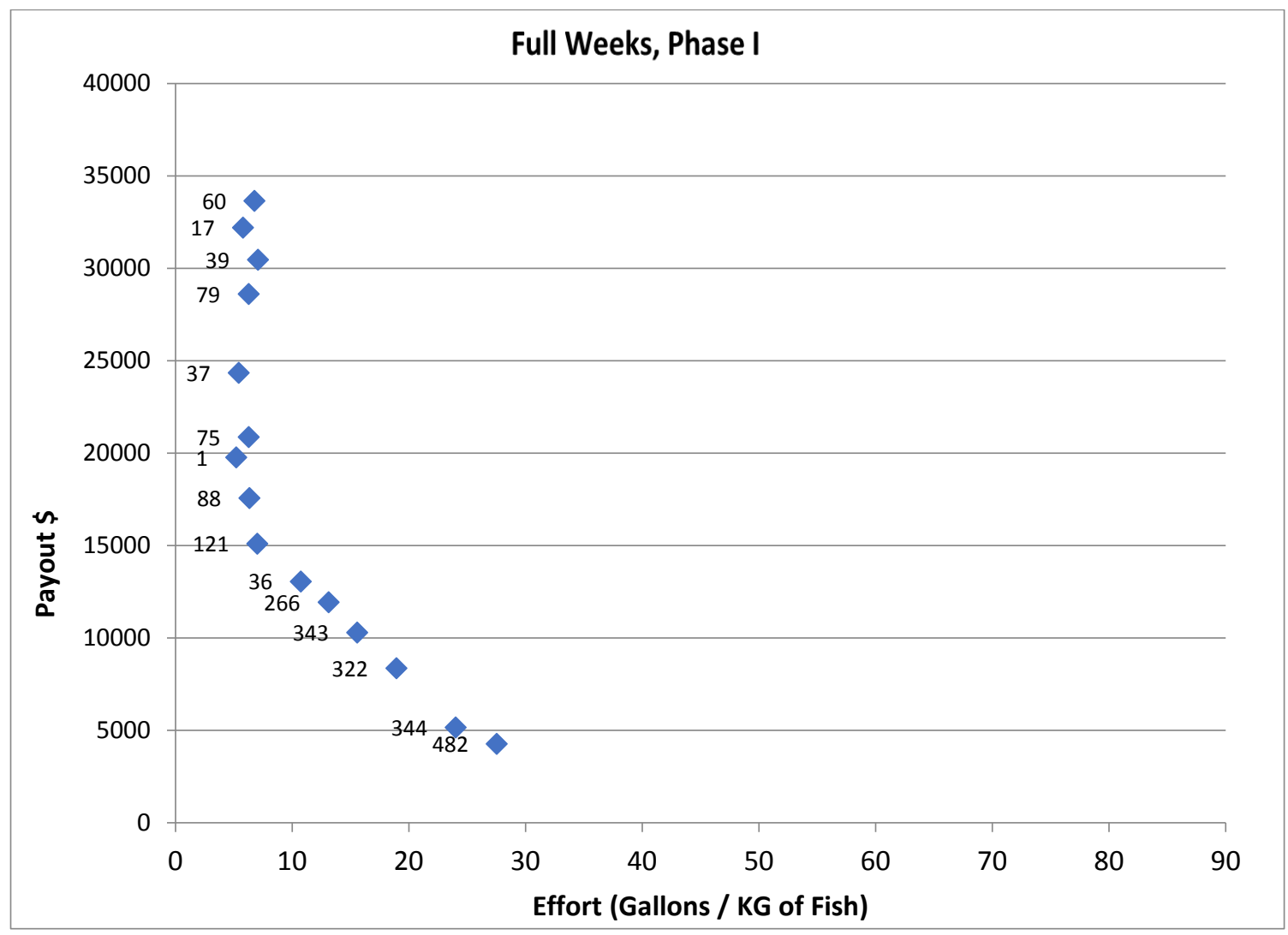

Figure 5.3: An example Pareto Front for Payout and Effort.

We will generate the corresponding Pareto fronts for a variety of scenarios in each of the three phases and use them as vehicles in order to describe how in general artisanal 
fishermen respond to each of the phases of ENSO. In the following section, 5.2 we provide the basic model that we will be using here. Section 5.3 then gives our conclusions.

\subsection{BASIC MODEL}

The key to the model is that while the results of each trip in terms of the catches are provided, there is little in the way of description as to who made these decisions. One cannot say that trip 68 and 124 were based upon the decision of a particular unit. So one of the basic assumptions that we need to make here is that a sequence of trips is associated with the decisions of an equivalence class of like-minded decision-makers where the identity of any particular decision-maker is not important.

Each category of agents will be asked to generate a sequence of trips within a given Phase. The three basic ENSO phases and their durations here are:

Phase I: El Nino Data collected from March $1^{\text {st }}$ to June $30^{\text {th }}, 1984$.

Phase II: La Nina, Data collected from July $1^{\text {st }}, 1984$ to July $31^{\text {st }}, 1985$.

Phase III: Back to Normal, Data collected from August $1^{\text {st }}, 1985$ to July $31^{\text {st }}, 1986$.

This sequence of trips for a given phase will be called a tour. A tour will begin on the first day of the Phase and end on the last day. The parameters used to guide an agent's decision will be:

1. The extent to which they wish to follow goal \#1 (profitability) vs. goal $\# 2$ (sustainability).

2. The days of the week that they wish to fish (depart out).

3. The downtime between trips. For our experiments here we assume that the agent makes just one trip out a day (does not go out fishing again on the same day they returned). 
4. All of the sites reported by the fishermen on their return will be used in the model, although subsets of sites could be used in future work.

Our analysis in Chapter 3 suggested that there was a learning curve associated with the trips taken, that results from prior day's experiences were used to focus the decision-making in terms of both location and catch. So four basic fishing scenarios were proposed in response:

1. Full Week. The agent would generate a tour decision for every day of the week, Monday through Sunday for all of the weeks under consideration for a Phase.

2. No Sundays. Culturally, some agents would not wish to work on Sundays, so those agents will generate tours for Monday through Saturday for each week in a Phase.

3. Mon_Tue_Wed. In our analysis in chapter 3 there were a number of instances where there was a trend from Monday through Wednesday. For some catches such as Lorna that trend was increasing. For other catches, especially highly targeted ones, the trend was decreasing from Monday through Wednesday. In other words, their priority was to harvest them first whenever possible.

4. Thur_Fri_Sat. We also observed that there was often a second wave of fishing activity in the latter half of the week for some catches and for some phases. Our goal is to see whether there were differences in these waves in terms of goal direction.

So having specified the days and phases over which an agent can make their decisions, the next question is how to operationalize the two goals. We begin first with profitability. Here, we will use Payout to represent Goal \#1 performance. For a given trip our model payout is the number of catch items times the relative worth of those items. Recall that in Chapter 3 we classified catches in terms of three categories of desirability. Those that were 
highly regarded for their commercial value (High), those that were of medium desirability from a commercial sense (Med), and those that were Fall Back catches- those that were not initially targeted for a trip but are still necessary to produce a successful catch when the others are not present. In our Payout function below we use a multiplier for the count of the catch based upon the desirability of that catch from a market standpoint. The Payout is not meant to reflect any specific currency, but the relative value of the catch produced by a trip.

Payout $=$ Catch Count $*$ Desirability

where High Des = 3, Med. Des = 2, Low Des $=1$.

The goal of sustainability was expressed in terms of an effort function. The contributors to effort were as follows:

1. Round trip distance (in KMs) between the port of origin, assumed to be Cerro Azul, and the site where the catch was made.

2. Fish weight in (KGs). That will influence the effort taken to transport it back to port. The greater the catch weight the more power needed to do so.

3. Miles per Gallon. While the boats used are all much smaller than commercial vessels, they do vary in size and capacity and therefore require engines with different power requirements. We selected a MPG value that reflects a middle ground in terms of engine power. One that would be a reasonable approximation of a range of motors that the agents might possess.

4. Relative Effort is a multiplier that adds some additional resistance to the journey. If the trip is for Cerro Azul and vicinity, then the round trip distance is the not adjusted. If the trip involves travel up or down the coast and away from Cerro Azul we added a 
simple multiplier to reflect the additional effort that would need to be made in those situations with regards to weather, currents, etc.

5. Fish weight: While profitability was expressed in terms of catch count, effort needs to be expressed in overall weight. So for a given trip the weight of the catch was used to predict effort while the count predicted profitability.

The Effort performance function is then simply the distance divided by the miles per gallon times a relative effort booster to reflect other hidden cost, divided by catch weight in KG.

Effort $=(($ Round Trip Dist. $/ \mathrm{MPG}) *$ Relative Effort $) /$ Fish-Weight

Where Relative Effort (RE) $=1$ for Cerro Azul, CA, and $3=$ for North or South $\mathrm{MPG}=5$.

Now that we have our measures for trip performance we need to parameterize the decision making for a given agent. We do so in the following way. Each decision strategy characteristic of a class of decision-makers can be expressed in terms of the percentage of time that they are driven by increasing Payout (Profitability) relative to minimizing effort (Sustainability). So a decision-makers profile can be viewed as a pair of probabilities:

(Probability of Maximizing, Profit; Probability of Minimizing Effort)

These probabilities for any tour produced by a decision-maker will add up to 1 . So $(100,0)$ means that the decisions-making agent will always select the trip with highest profitability. Likewise, $(0,100)$ means that the trip that uses minimal effort is that one that will be selected at every choice point along the tour. The day constraints determine what choice points an agent can visit during the search. 
This allows us to generate a simple plan to deal with the bi-objective problem here.

In Chapter 6 we will generate 500 such random strategies and use each to generate a tour though the database of trips. For a given tour, a Profit/Sustainability profile is determined. The set of days over which fishing can take place for a given phase is turned into a search graph. The idea is that at the start of the tour the agent must select between the tours that go out that day based upon the effort and the profitability. A $(100,0)$ agent will always select the trip that maximizes profit for the day, while the $(0,100)$ agent will focus on the one that will utilizes the least effort so as to insure sustainability.

A tour for a given strategy is a sequence of trips selected from day to day in the search graph associated with the days and the Phase over which the tour is defined [40]. An example of this is given in Figure 5.4 below. This gives a portion of a generated tour for the first few days of the Residual El Nino for the ALL Days option. In this tour the agent has selected to Minimize Effort $100 \%$ of the time. When the values are too close together (less than a certain distance) a random number generator (Mersenne Prime) is used to select the appropriate trip. Then, at the next day again a random number generator is used to break a second tie, and add it to the tour. This process while continue until the end of the Phase. The effort and Payout associated with the tour is then returned as a result. If the strategy was (50, 50) then at each step a random number would be generated and each would have an equal chance of selection. Therefore, any combination of goals can be achieved via this schema. 


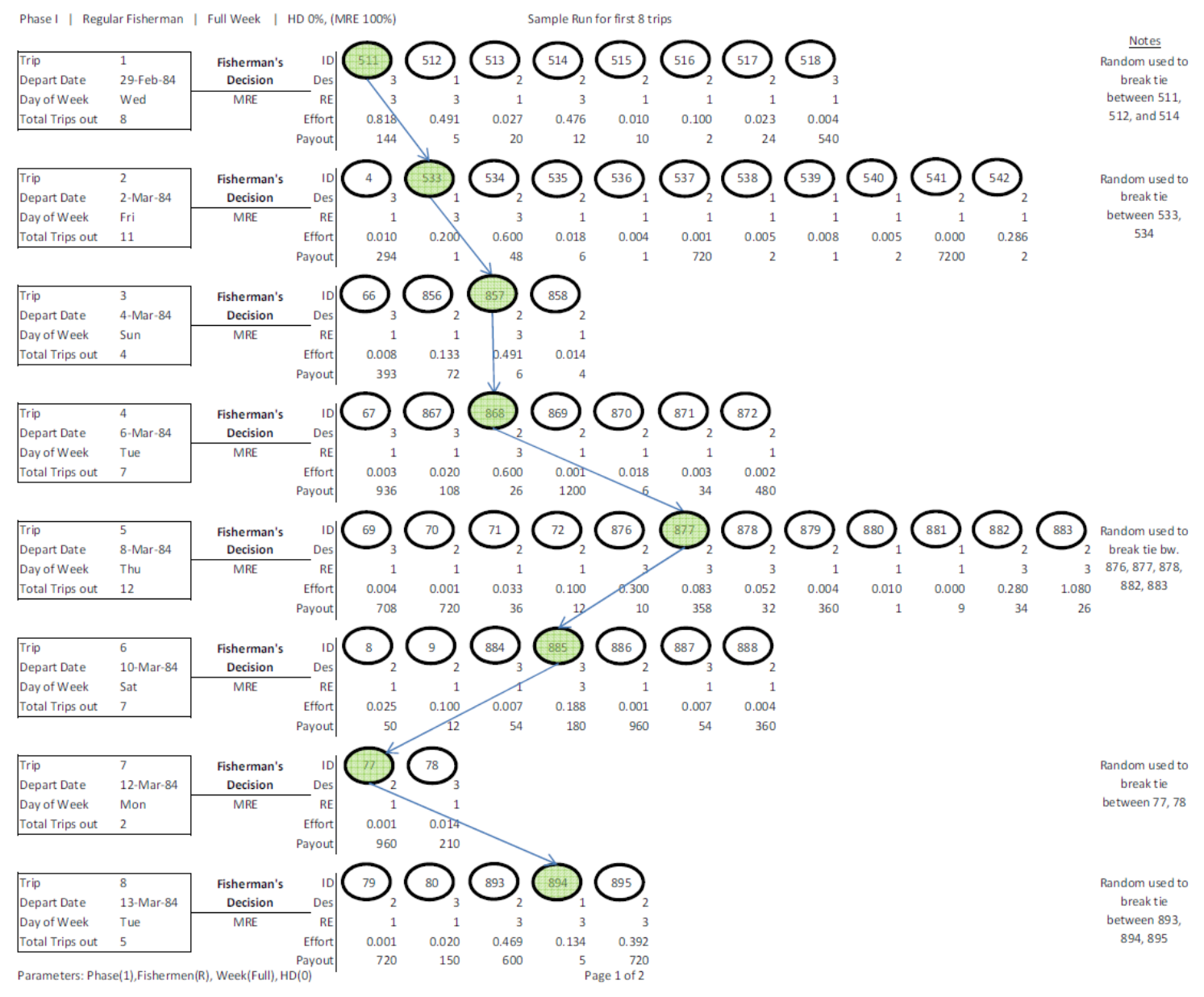

Figure 5.4: A Decision Tree of the sample tour of the first 8 days using $(0,100)$

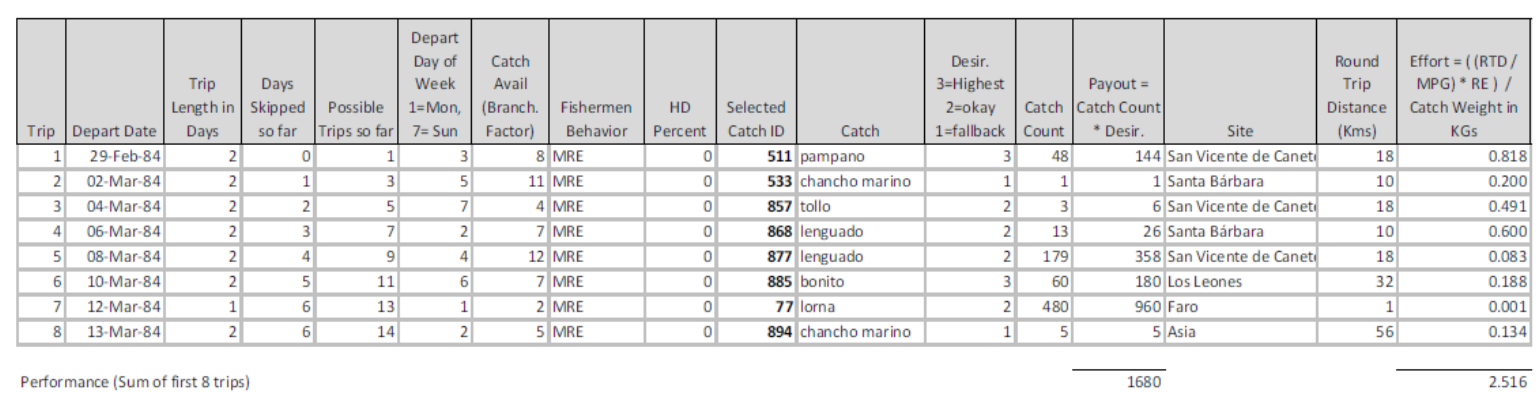

Figure 5.5: Details about the Decision Tree of the sample in Figure 5.4 
So the performance of a tour for a given Phase/Day scenario is the sum of the profits and the efforts over all of the selected trips. Those points are then plotted and used to produce the Pareto curves discussed in the next chapter.

\section{2: CONCLUSIONS.}

We have modeled fishing agent decision making as the process of generating a trajectory or path through a search graph based upon the agent's relative goals. In other words, the simulation can be viewed as a graph search process defined over the set of all possible trip sequences in order to express the differential impact of the two conflicting goals on over agent behavior. This will allow us to assess the impact that the changing dynamics of the ocean environment has on the ability of different decision strategies to work effectively. That will be the topics of the Chapter 6 . 


\section{CHAPTER 6: THE EXPERIMENTAL RESULTS}

\subsection{INTRODUCTION}

In this chapter, we will conduct a thorough investigation to compare and assess the outcomes for several scenarios as previously discussed in chapter 5 . We designed the fishing model as a bi-objective model by using two criteria based on the data availability and fishing activities. We plot the effort on the $\mathrm{x}$ axis and payout on the $\mathrm{y}$ axis:

Effort $=(($ Round Trip Dist. / MPG) $*$ Relative Effort $) /$ Fish-Weight; Where Relative Effort, RE $=1$ for trip to Cerro Azul, CA, $3=$ for South, $3=$ for North and $\mathbf{M P G}=\mathbf{5}$.

Payout $=$ Catch Count $*$ Desirability, where High $\mathrm{D}=3, \mathrm{Med}=2$, Low $=1$.

Our objective is to find a set of solutions that reflect the tradeoffs between the two conflicting goals relative to We ran the model for each of the three different ENSO phases;

1. Phase I: El Nino Data collected from March $1^{\text {st }}$ to June $30^{\text {th }}, 1984$.

2. Phase II: La Nina, Data collected from July $1^{\text {st }}, 1984$ to July $31^{\text {st }}, 1985$.

3. Phase III: Back to Normal, Data collected from August $1^{\text {st }}, 1985$ to July $31^{\text {st }}$.

In multi-objective optimization problems, the goodness of a solution is determined by the dominance of solution vectors within a feasible region. The feasible region is defined by the set of the constraints. Here, we generated 500 random independent tours for each Phase. It was felt that given the structure and branching factor the search graph that the simple generation of a relatively large number of points, and then applying non-dominated sorting to produce the resultant curve would be sufficient for our purposes in this Chapter. In the follow-up Chapter our need to statistically characterize these distributions in more detail leads us to use the Cultural Algorithm described previously in Chapter 4. 
To evaluate and assess the results we present a comparison of twelve distinct fishing scenarios defined in terms of the ENSO phase and days. For every Phase there are four different models in terms of the days of the week over which the tour was operated. We will use the fisherman model where the agent cannot go out again the day that they come back from a fishing trip.

First, we plot the real values of the outcome for the multi-objective function for the fishermen trips. We have randomly generated 500 tours of the tour graph in order to calculate the payout and the corresponding value for the effort. Each point $(\mathrm{x}, \mathrm{y})$ in the graph represents a result of a tour, where $\mathrm{x}$ are the Effort and $\mathrm{y}$ are the Payout respectively for each tour.

\subsection{COMPARISON OF PARETO FRONTIERS FOR PHASE I}

To compare and assess the results we begin with a comparison between each of four scenarios for Phase I. Each scenario corresponds to a sequence of trips generated in the phase by fishermen using a particular strategy in terms of the two goals. We begin with the Full Week Scenario where every day of the week is an available stop on the fishermen's itinerary for the given phase.

Figure 6.1 gives the Pareto Frontier for Fishermen whose tours can take place on all 7 days of the week in Phase I, the Residual El Nino. Notice that in this Phase the presence of targeted fish dominates the need to invest in more resources to achieve a successful trip. Recall that in the base case for Effort, a successful trip is one that brings back a catch. All of the trips in our database represent successful trips in that regard.

What this curve means is that many targeted catches can be found within a short distance from Cerro Azul during the time of March through June. This is the conclusion of 
the El Nino which is moderated by the fact that it is the tail end of summer and beginning of fall. Warm water fish are enticed to remain in the area even though the warming phase of El Nino has diminished. It suggests that a productive sequence of trips in terms of Payout will be more dependent on timing than on location. Once fishermen are required to put more resources into the tour in this Phase, the Payout drops exponentially.

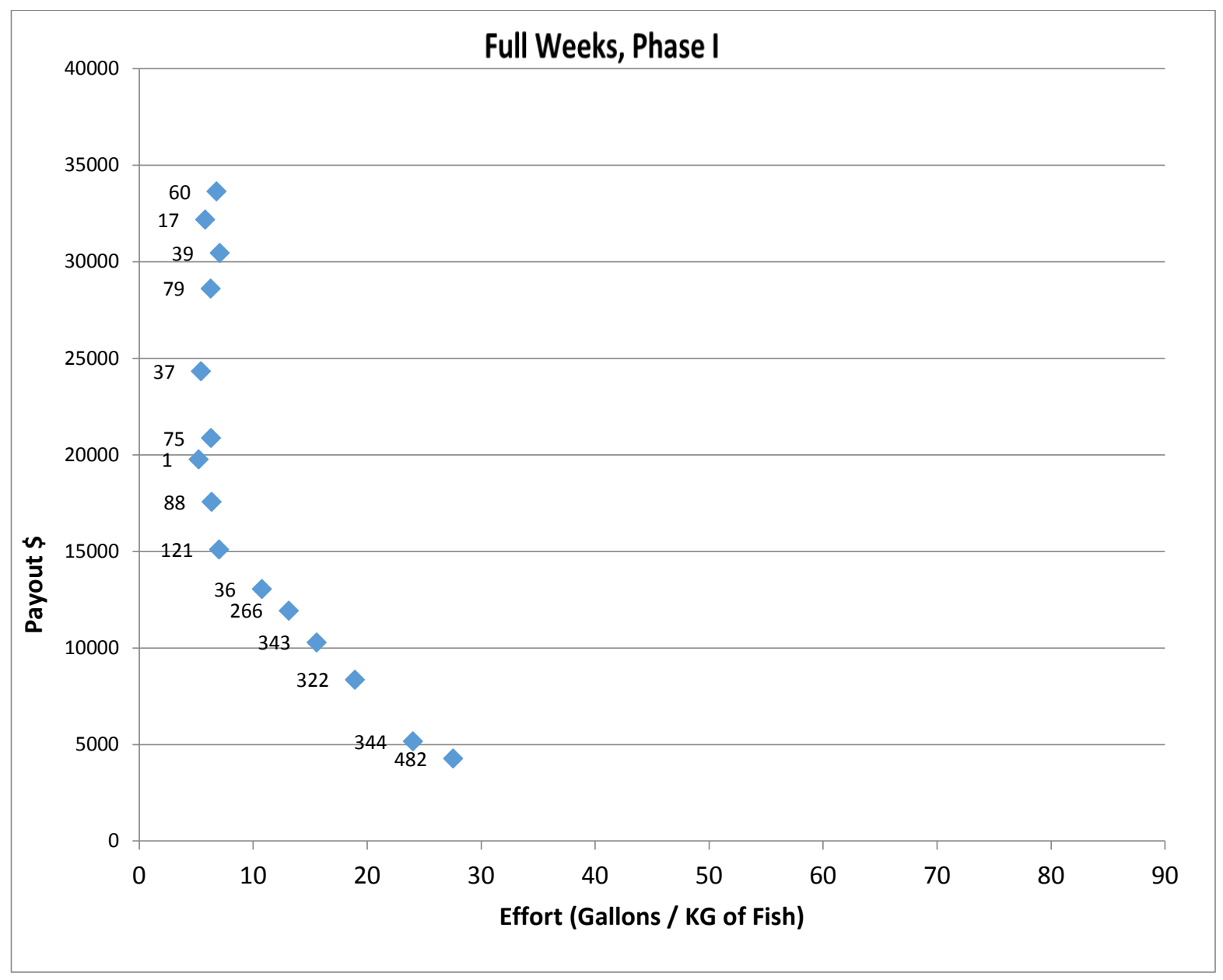

Figure 6.1: Pareto Front for Payout, Effort, FK Phase I- EM 


\section{FullWeek, Phase I}

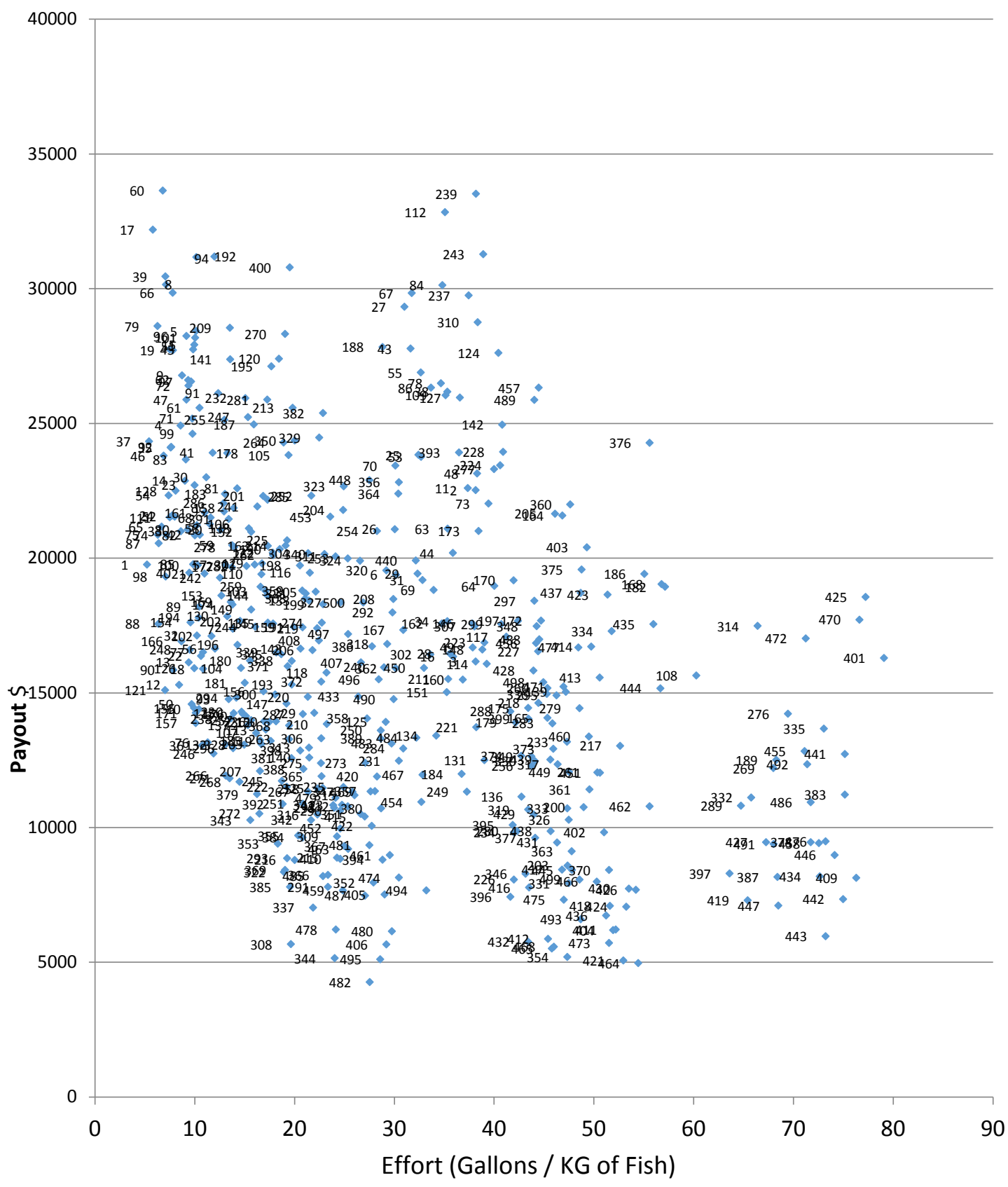

Figure 6.2: A plot of 500 tours generated for the Pareto Front - Phase I Full Wk 
In Figure 6.2 there seems to be a potential second front as well but it is dominated by the number of tours associated with the main front and connected by an intermediate set of tours that occur around an effort of 30 . In order to investigate this phenomenon in more detail we studied the tours that are produced by agents that do not use Sundays to fish. Sundays are problematic anyway since many fishermen consider that to be a day or rest. On top of that the government official may not always be available to count catches on that day as well.

Next, we present the Pareto front for tours produced by our agents when Sundays are not used for tours. In this case an interesting result appears. When plotting the generated points for these tours, one can see two relatively distinct fronts emerging there (Figure 6.3). Figure 6.3 shows a plot of 500 points of the real values of the Effort on the $\mathrm{x}$ axis and the Payout on the y axis. There are clearly two fronts that have emerged. 


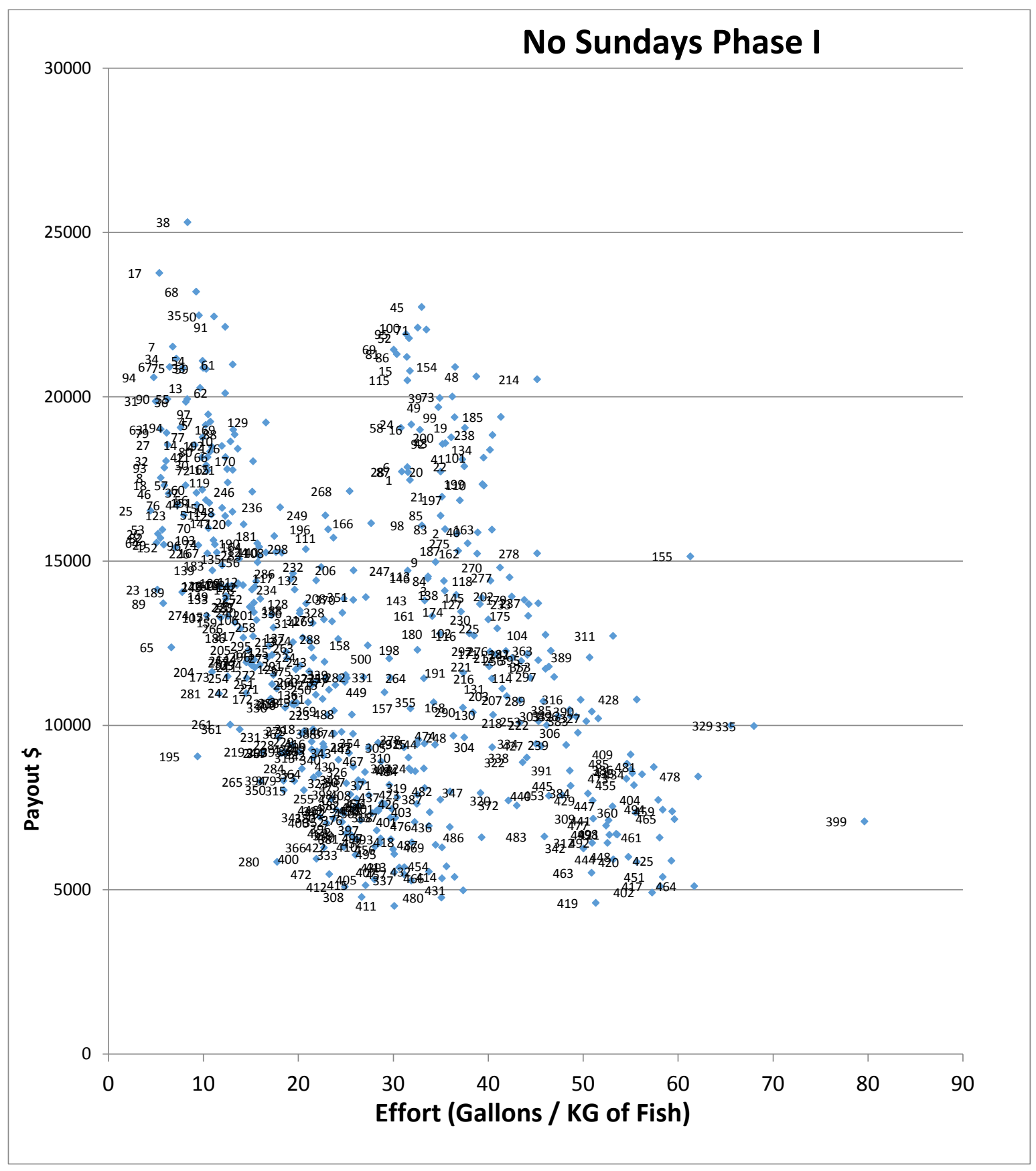

Figure 6.3: Plot of 500 Tours that contain No Sundays for fishing agents in Phase I.

Figure 6.4 shows the two Pareto Fronts for Payout Versus Effort, for No Sundays Phase I. The first and most dominant front exhibits high returns on targeted catches which 
decrease exponentially as the effort required to produce a fishing expedition increases. The second less dominant front can produce yields that rival the first one but at the expense of more effort.

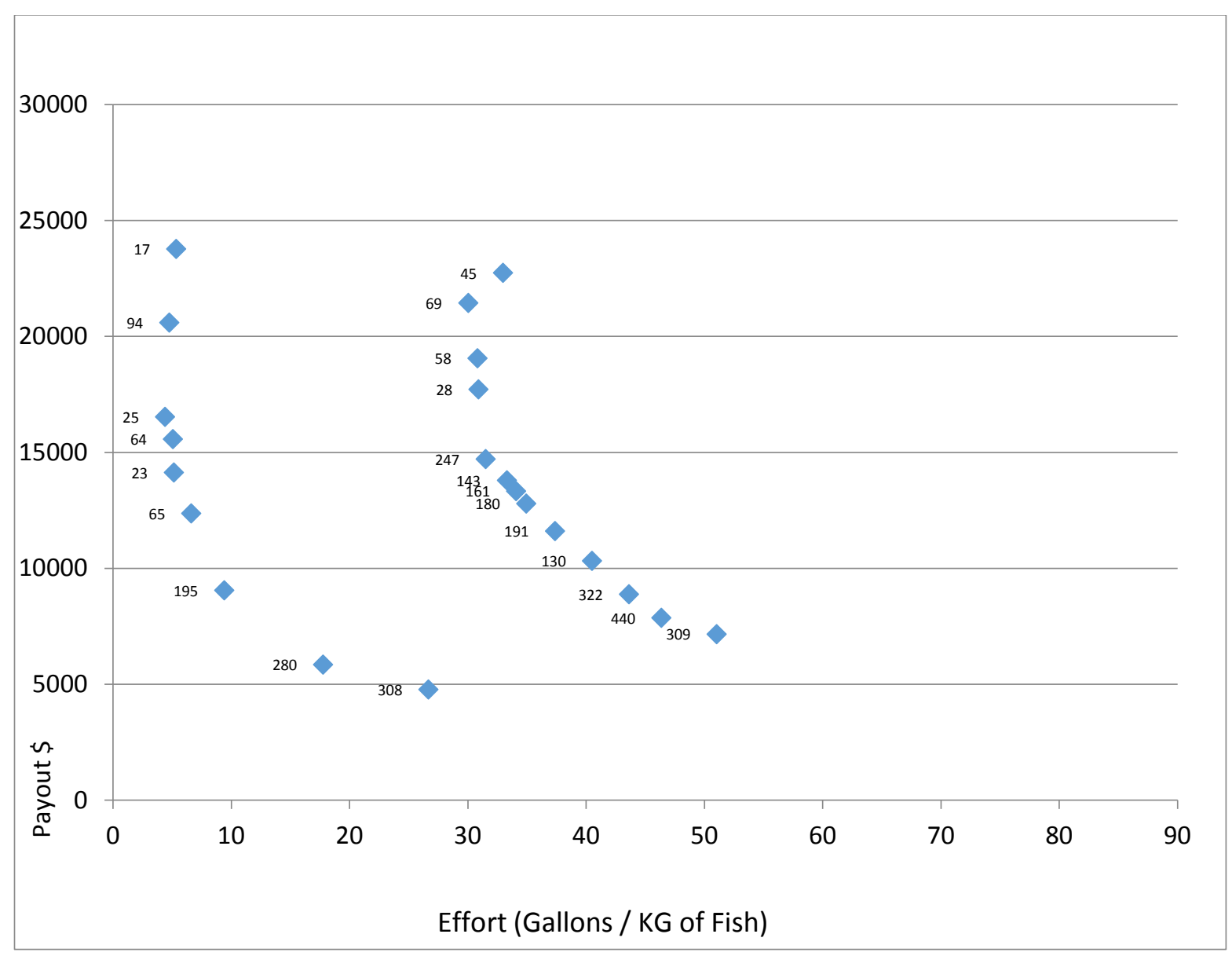

Figure 6.4: The two Pareto Fronts for Payout, Effort, Phase I, No Sundays

Next, we present the graphs for $1^{\text {st }}$ half of the week: Monday, Tuesday, and Wednesday scenarios in Figure 6.5. Note that these agents are restricted to less than half of the days that are available so the yields will reflect that reduction along with the resources used. However, the top yields of 12,000 are just about half of the No Sundays best which is good for just three days out of the week. 
The corresponding Mon_Tue_Wed Pareto front is given in Figure 6.6. It exhibits an exponential drop like the No Sundays initially, but the addition of more resources does effect to moderate that drop. This moderation suggests that there is some learning going on between agents during that period that is information is being exchanged between agents in order to allow some prediction as to where to fish in the following day. 


\section{Mon, Tue, Wed Phase I}

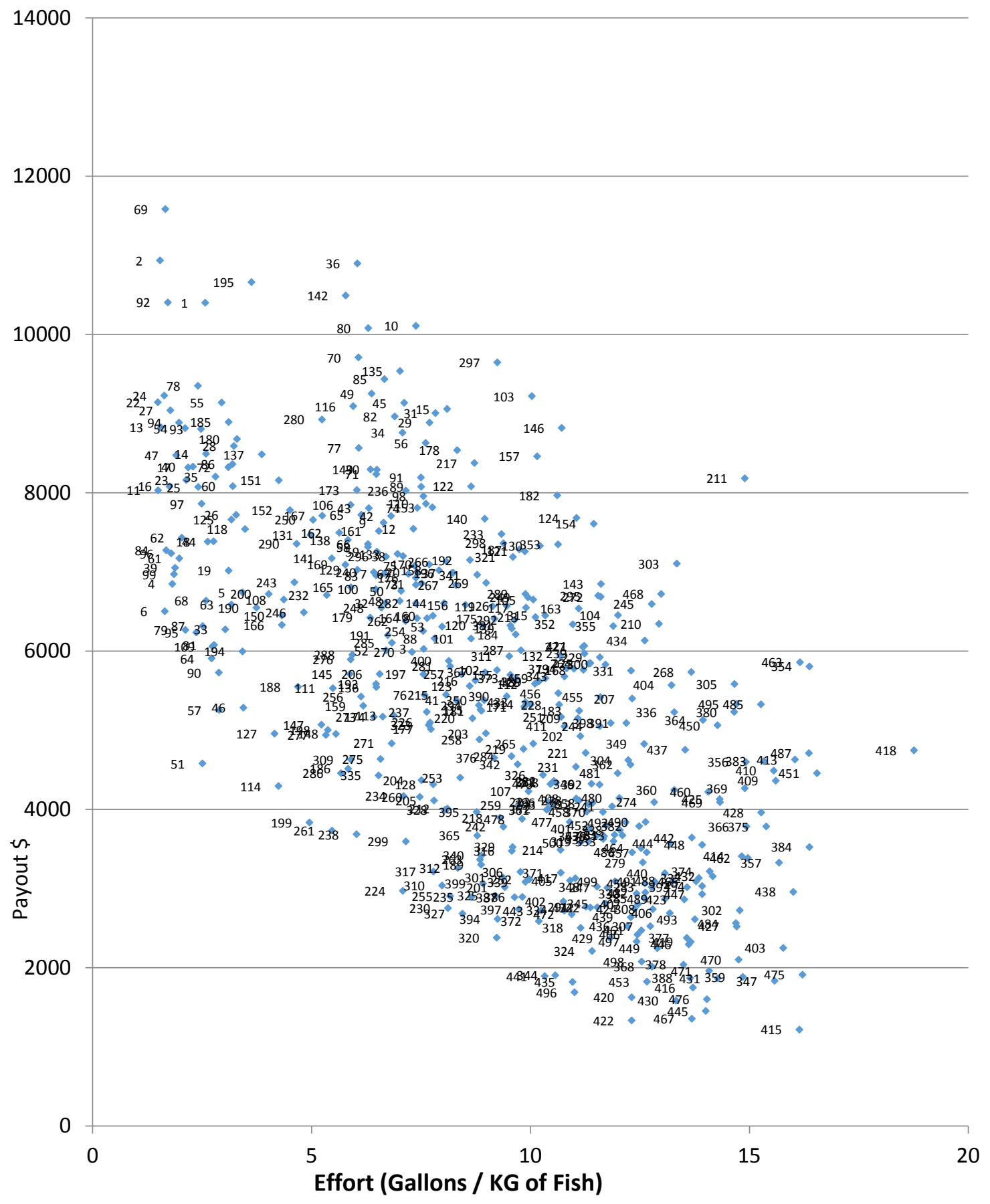

Figure 6.5: Plot of 500 points of the values $M_{-} T_{-}$W Phase I fishermen. 


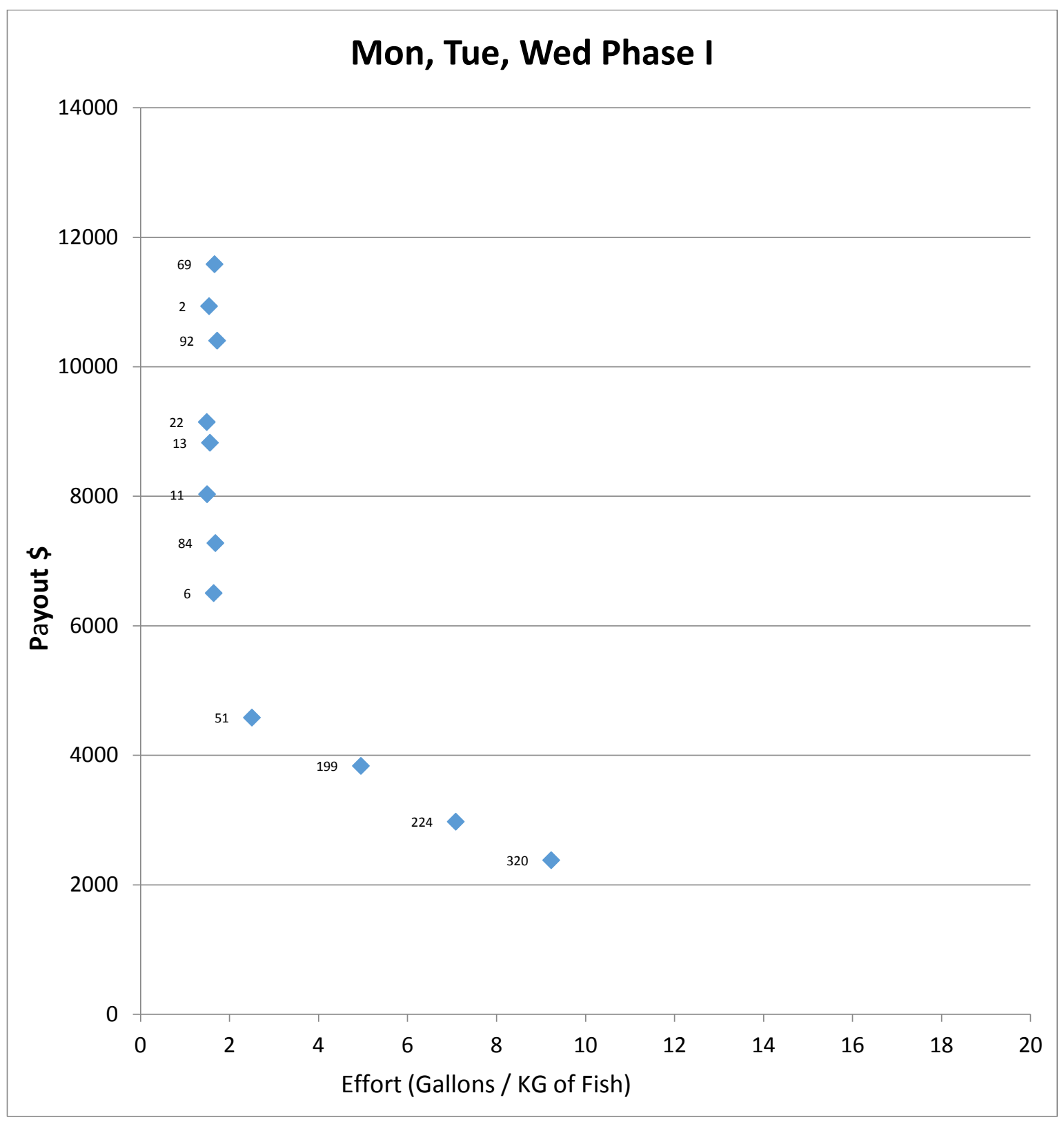

Figure 6.6: Pareto Front for Payout, Effort, Mon_Tue_Wed Phase I. 
Next, we present the graphs for $2^{\text {nd }}$ half of the week, Thursday, Friday and Saturday scenarios. Figure 6.7 shows a plot of 500 points of the real values of the effort on the $\mathrm{x}$ axis and the payout on the y axis. Figure 6.8 shows the Pareto Front for Payout [ $\$ / \mathrm{Kg}$ of fish] Versus Effort [Gallon $/ \mathrm{Kg}$ of fish], for $2^{\text {nd }}$ half of the week, Thursday, Friday and Saturday, Phase I with High Desirability.

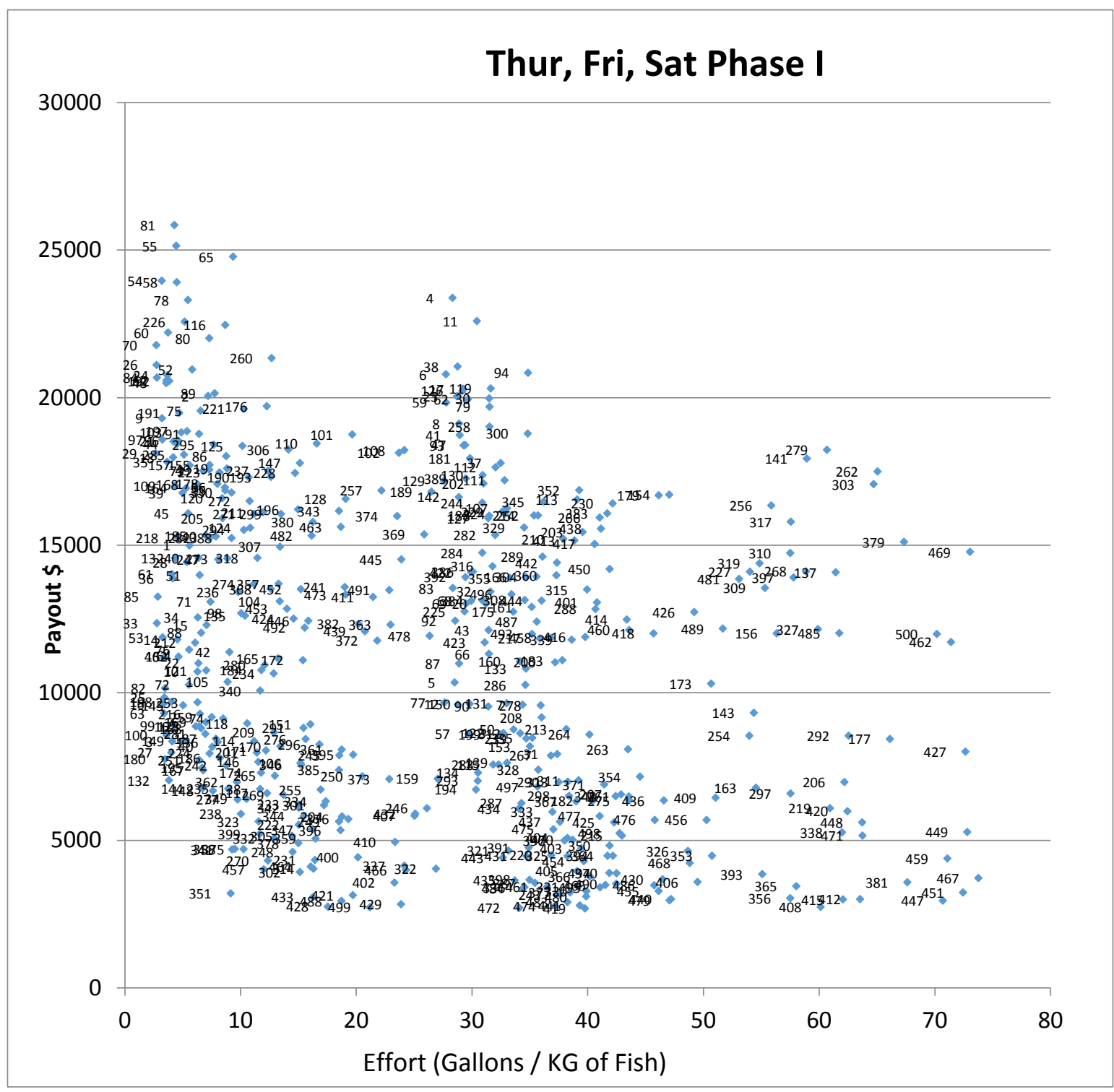

Figure 6.7: Plot of 500 points of the real values RFS Phase I 
Figure 6.7 shows a plot of points of the real values of the effort on the $\mathrm{x}$ axis and the payout on the y axis produced during the search. What is interesting here is that there appears to be three possible fronts there. These fronts are each yielding more than the M_W_F fronts even though those are just three days in length. However, they require the expenditure of more effort. That is, they must search progressively further from Cerro Azul as the week goes on. It is likely that each curve reflects trips that start on Thursdays, Fridays, and Saturdays respectively. Saturdays have fewer agents but it still produces comparative yield although with more resources needed.

The Pareto front for the dominant front is given in Figure 6.8. That curve comprises the largest number of agent tours but exhibits a sharp decline as one moves away from Cerro Azul. This suggests that fishing grounds are moving elsewhere towards the end of the week. The fact that these yields later in the week are still high it means that they are going to areas further away that are likely to yield good results. There are clearly several emergent relationships between agent decision-making. It will be interesting to see how the onset of $\mathrm{La}$ Nino will impact these patterns. 


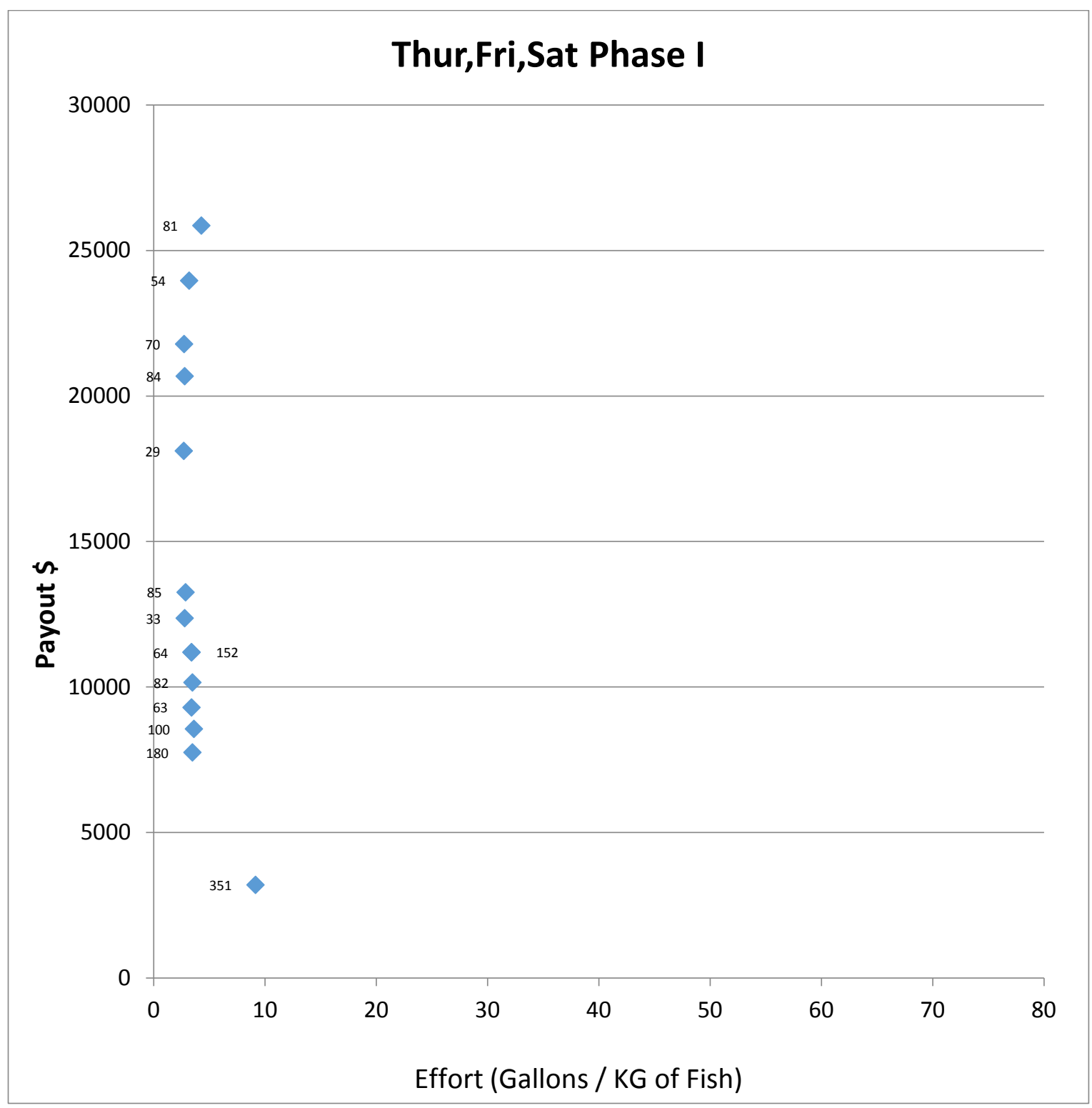

Figure 6.8 Pareto Front for Payout, Effort, Thur_Fri_Sat Phase I.

\subsection{COMPARISON OF PARETO FRONTIER FOR PHASE II.}

In the previous section we observed the emergence of several Pareto wave fronts, the most dominant occurring at the start of the week Mon_Tue_Wed and then trailing off with less payout and more resource investment as the week went on. In this section we will 
investigate Fishermen's decision-making behavior in response to the cooling of the ocean in La Nina.

In Figure 6.9 a plot of 500 randomly generated tours is provided. Notice that the Payout produced is in the range of three times that for the residual El Nino. The Effort on the other hand extends to almost 10 times that of the El Nino. However, recall that the tours for La Nina extend our almost a year while those for El Nino are over a quarter of that time. So if we multiply those El Nino by three we approximate the Payout for La Nina, but clearly the effort that are need to produce this yield have increased. Agents now need to search a wider range of sites to the North and South of Cerro Azul proper. 


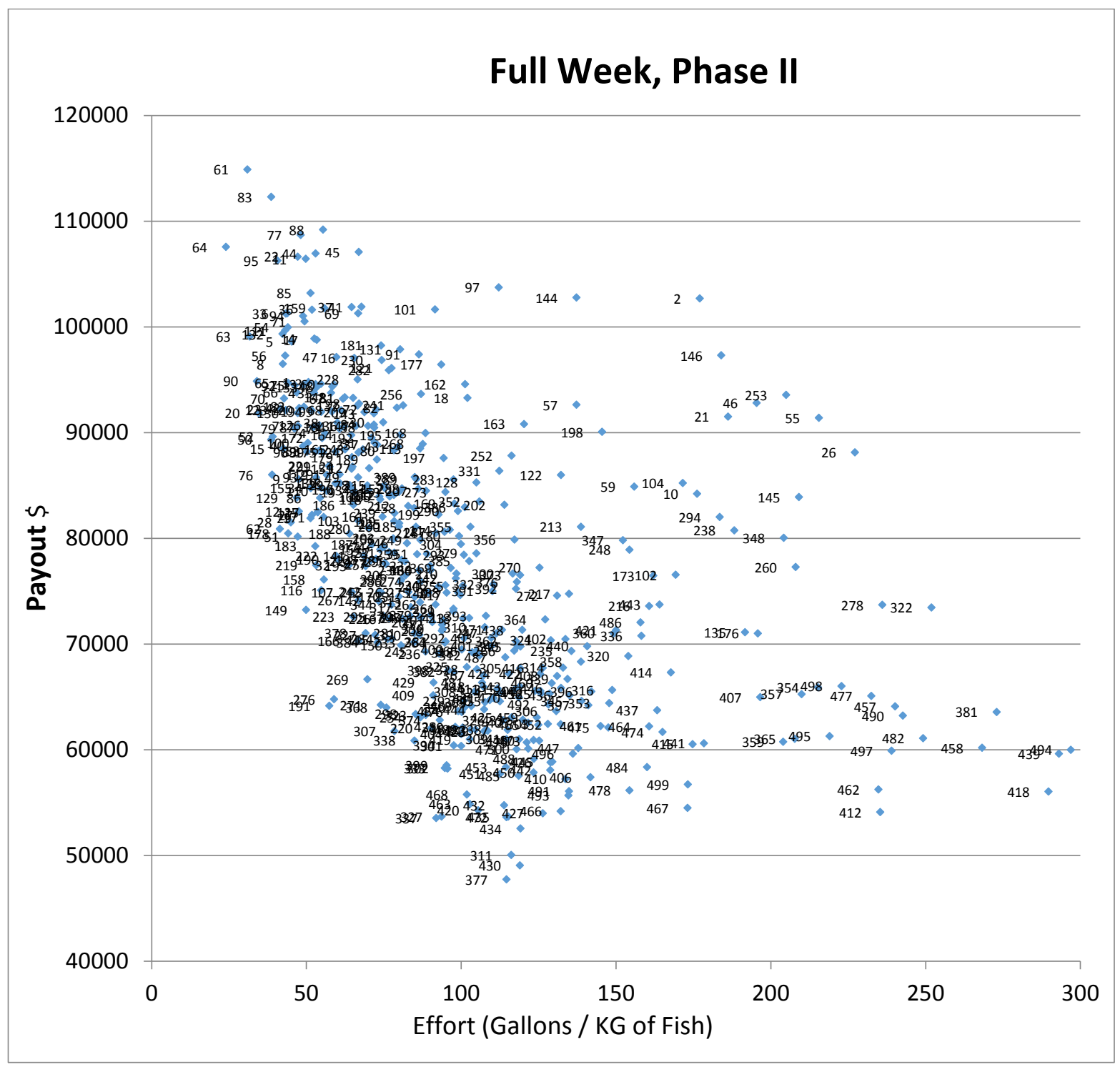

Figure 6.9: Plot of 500 points of randomly generated tour values for Full Wk in Phase II

The corresponding Pareto Front is shown in Figure 6.10. Notice that the main front is shifted upwards in terms of Payout and stretched horizontally in terms of resources used to produce a successful trip. Even for those trips that required larger amounts of resources, the payout was better than that for the tail end of El Nino. In other words the perceived return on investment was definitely greater in this phase than for Phase I. The cooling waters were beginning to reestablish the food chains that supported a variety of new catches. In addition 
the warm water fish, although retreating, were still within travelling distance from Cerro Azul to the North. In addition, travel to the South allowed the agents to capture cold water fish as they begin to move to move north ward. So although there were limits to what could be caught in nearby Cerro Azul, there was more effort to go beyond the locality to exploit the newly available resources.

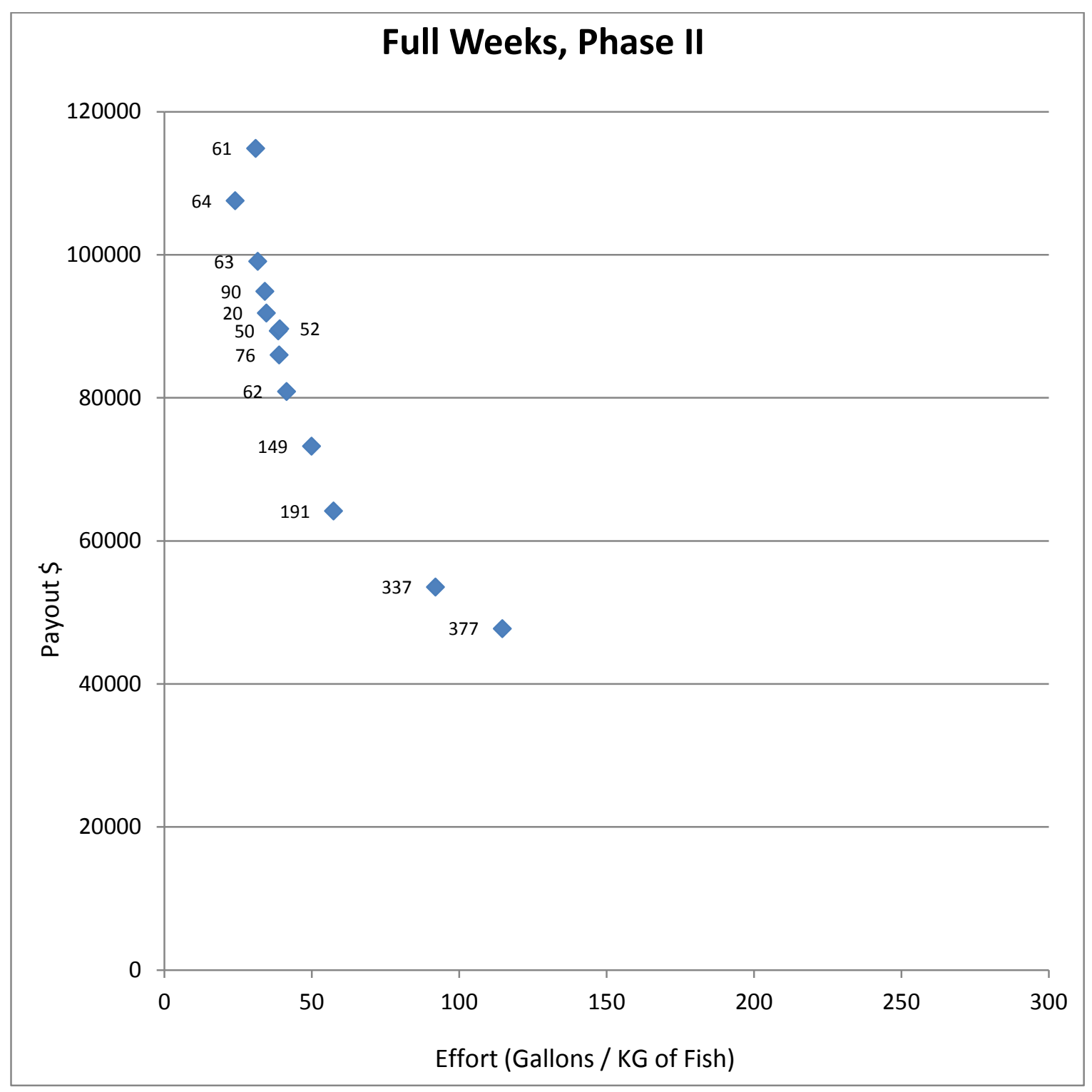

Figure 6.10: The dominant Pareto Front for Payout, Effort, Full week Phase II. 
Next, Figure 6.11 illustrates the distribution of generated points when tours that utilize Sundays are removed. Removing Sundays dropped the Payout down more than it did for Phase I. It suggests that fishing was lucrative enough to attract more activity in the La Nina Phase than in Phase I. While the distribution suggests a vestigial second front that required more resources, the main Front was clearly the dominant attractor for decision-makers here.

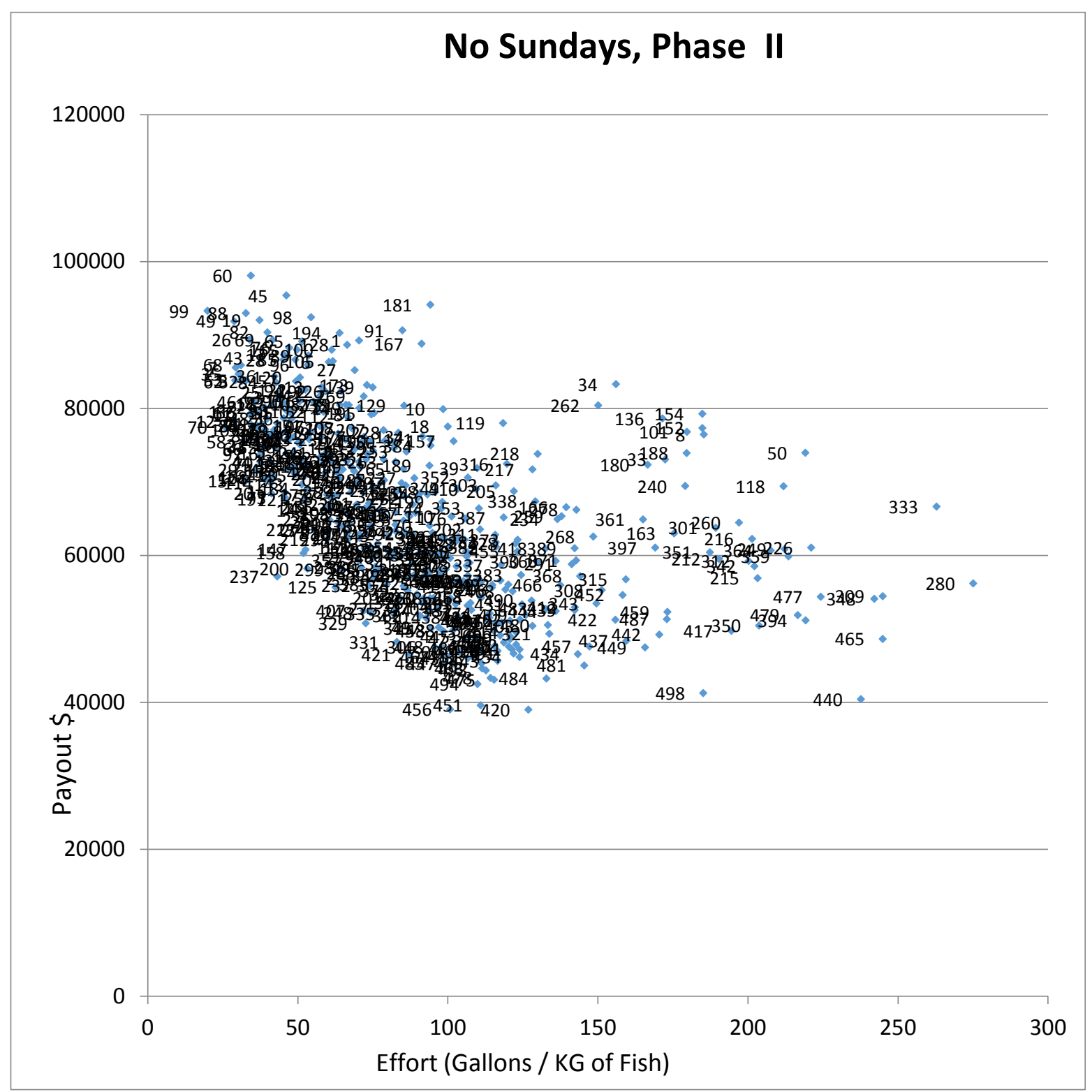

Figure 6.11: Plot of 500 tours for No Sundays in Phase II. 


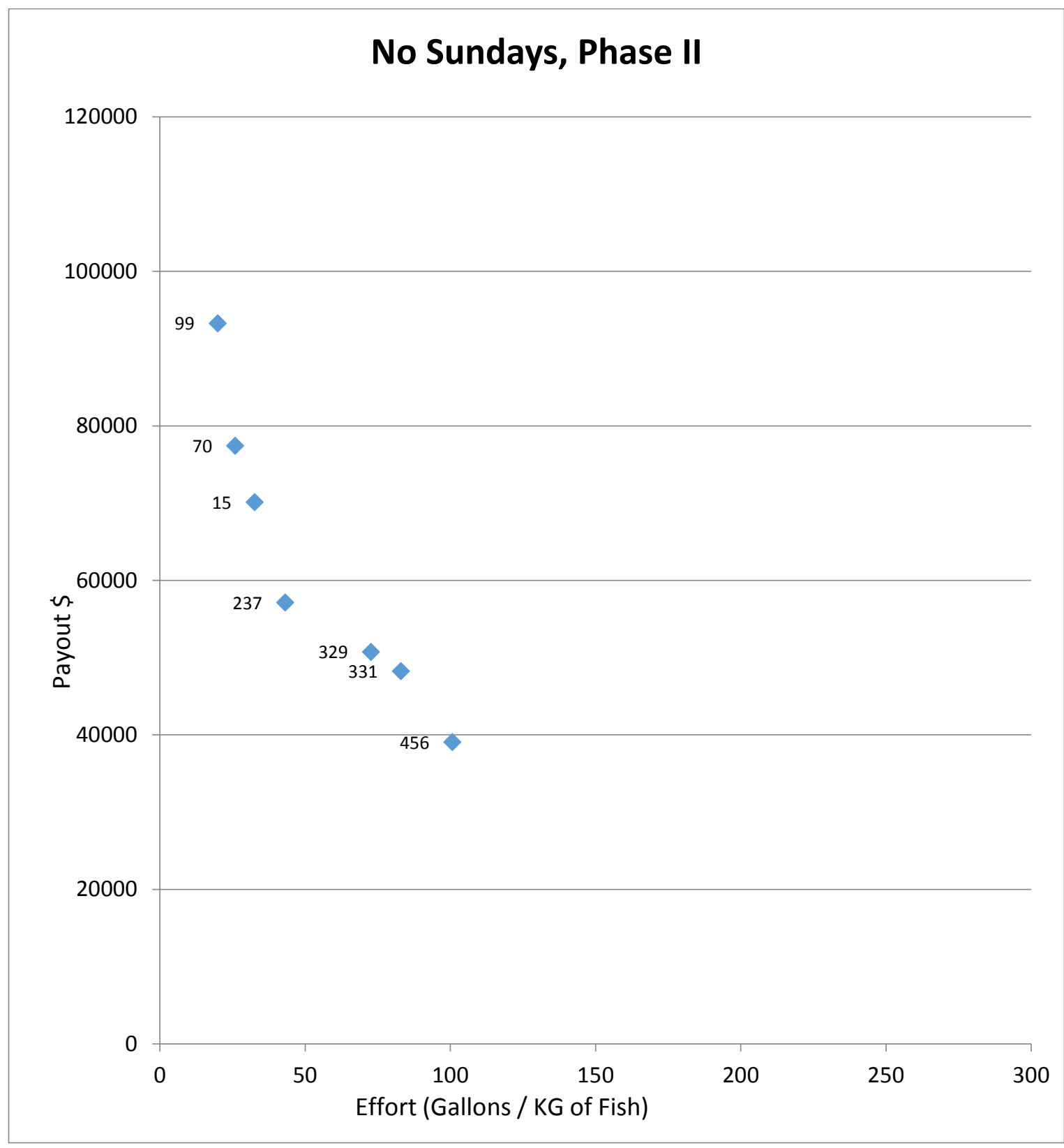

Figure 6.12: The Pareto Front for Payout, Effort for tours with No Sundays. Phase II

Figure 6.12 provides the Pareto front for the dominant distribution of tours given in Figure 6.11 above. The Payout and Effort have been reduced since Fishermen can no longer use Sundays to add to their tour values. The Maximum Payout is now just three times that of El Nino while the resource utilization for successful trips is now about twice that of the Phase I main front. Investing more effort in trips is producing almost an equal amount of return. 
Also, the curve now is more gradual when it comes to adding more resources rather than exhibiting the steep drop shown in Phase I.

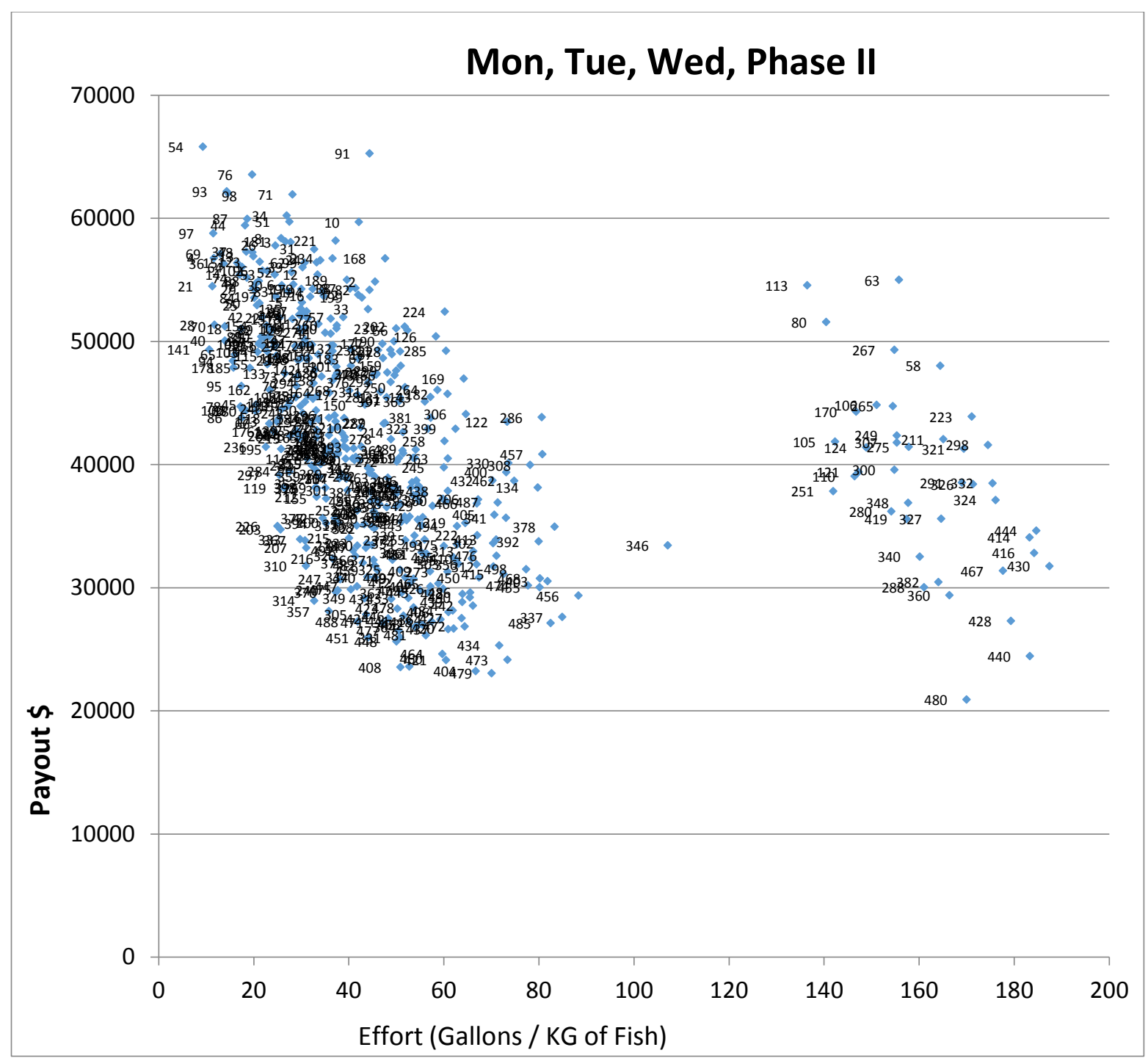

Figure 6.13: Plot of 500 randomly generated tours for just those that use MTW, Phase II.

Figure 6.13 exhibits a plot of the generated tours that use only departures from MTW. Most of them correspond to the dominant Pareto front and are able to achieve success relatively close to Cerro Azul. The Payout drops quickly relative to effort, but it is more gradual than for Phase I with regards to effort. There appears to be a secondary front 
associated with a group that expends high effort but achieves a Payout on the order of those that observe that the first front. Figure 6.14 shows the Pareto front for the main grouping. There, Payout is high but declines quickly with effort used. While expending more effort guarantees success and compatible returns it suggests that there is some priority or privilege that means certain individual or groups do not have priority access to the fishing regions around Cerro Azul and must go elsewhere to be competitive. 


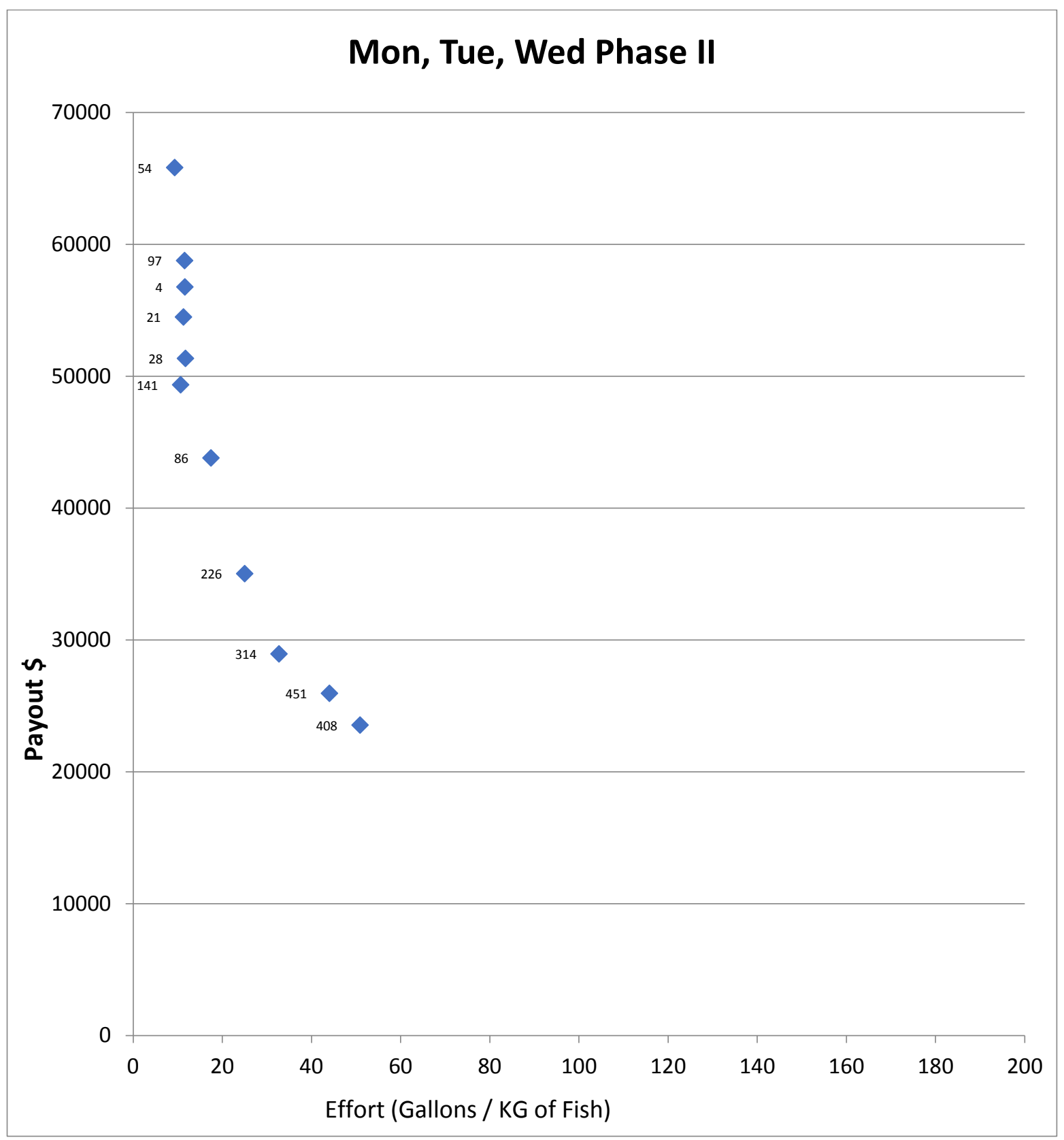

Figure 6.14: The Pareto Front for Payout vs. Effort for tours on MTW Phase II. 


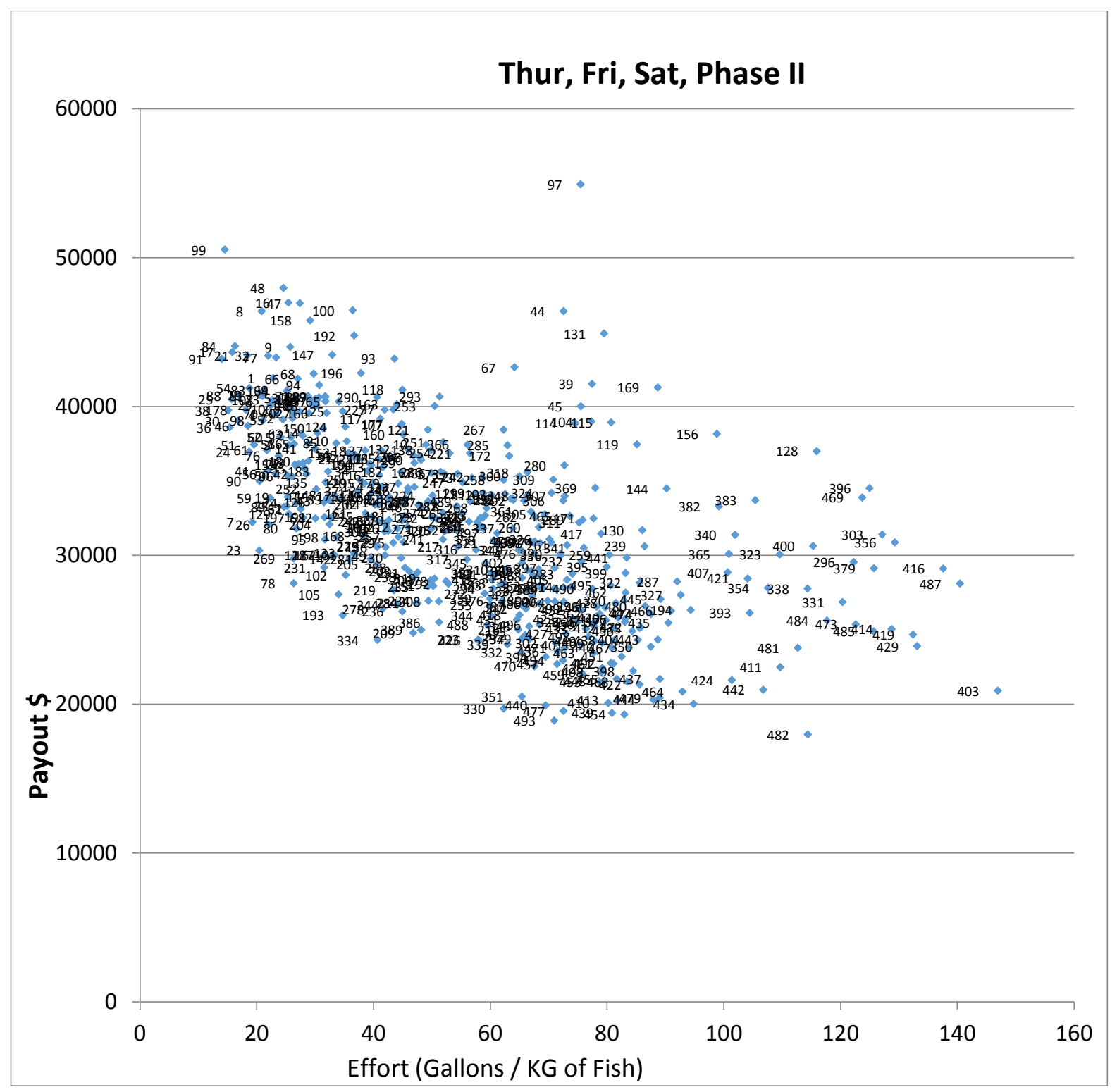

Figure 6.15: The Plot of 500 points for trips starting on Thur_Fri_Sat in Phase II.

The main distribution of points for Thur_Fri_Sat in Figure 6.15 is shifted down with regards to payoff and to the right with regards to resources relative to the M_W_F curve. Again there is a smaller secondary distribution to the right of the main one. If the Mon_Tue_Wed curve represents the tradeoff for the exploration process in this phase, the Thur_Fri_Sat may correspond to exploitation of found catch patterns. If that is the case, it 
means that information collected during the first half of the week is used to trigger exploitation in the second half. The Pareto front associated with the Thur_Fri_Sat is given in Figure 6.16. There we see that the corresponding front is indeed shifted down on the $\mathrm{Y}$ axis and to the right on the left. The tradeoff between the two goals is much more gradual than for Mon_Tue_Wed which suggests more flexibility in the search process.

Overall, the relative shape of the Pareto curves in Phase II suggest that in order to maintain the same Payout yield per month in La Nina relative to that for El Nino the corresponding Pareto fronts are stretched out over a larger effort range. That means that they need to cover a larger area to maintain their expected returns. In addition, as one progresses from M through Sat each new Pareto front has a Payout that is slightly less than the Phase before but requires more effort to achieve. In other words, in order to achieve the same profitability, the agents need to invest more and work harder in the process. 


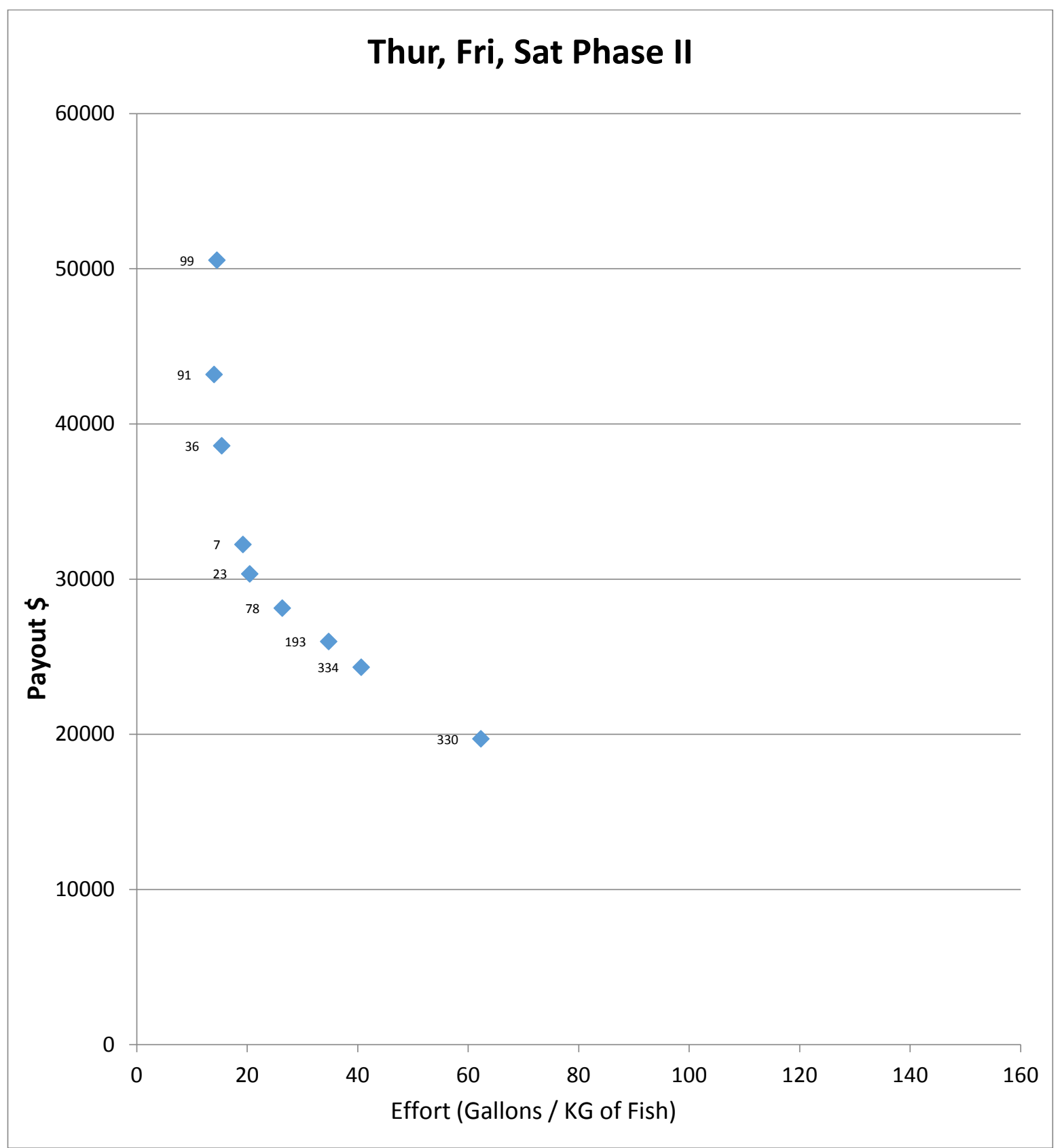

Figure 6.16: The Dominant Pareto Front for Payout, Effort, Thur_Fri_Sat Phase II

\subsection{COMPARISON OF PARETO FRONTIERS FOR PHASE III, BACK TO NORMAL.}

The transition between Phase I and Phase II as reflected in the Pareto fronts suggest that the goal of the agents want to maintain a similar per month Payout, but the mixing of warm and cooler waters provided more variety in terms of opportunities and required more 
search to obtain equivalent per month results. In addition, there was more emphasis on fishing earlier in the week than later in Phase II than I. Given the larger area for search there is more of an emphasis on the flow of information among fishermen explorative phases associated with first days of the week rather than later in the week. But the tours in the latter part of the week are fewer but produce equivalent Payouts but with additional outlays of resources. That is possible if individuals have a good idea of where to go. It suggests that there is an implicit communication channels that employs the results of earlier tours in the week to help focus tours later in the week. .

The distribution of randomly generated tours for the Full week in Phase III is given in Figure 6.17. Notice that the tours are bringing back Payouts similar in range to that of La Nina but the effort range is much more limited to around 50 units. Since these conditions are closer to their normal conditions there is less need for a wider range of search in order to retrieve a catch of sufficient magnitude. The agents are employing their traditional knowledge once again expending diverse amount of resources to search over a broader area for a diverse set of catches is no longer necessary.

Figure 6.18 gives the corresponding Pareto front for the entire week. When compared with that for La Nina the maximum Payouts are down somewhat but so are the resource expenditures required for a successful trip. There is a gap around 20 without any tours but the tail end picks up around 40. This indicates that rather than having a second Pareto Front that going larger distance on occasion was part of the natural cycle of things. Therefore, even the long distance trips were less of a gamble since they drew on knowledge of prior catch schooling behavior. 


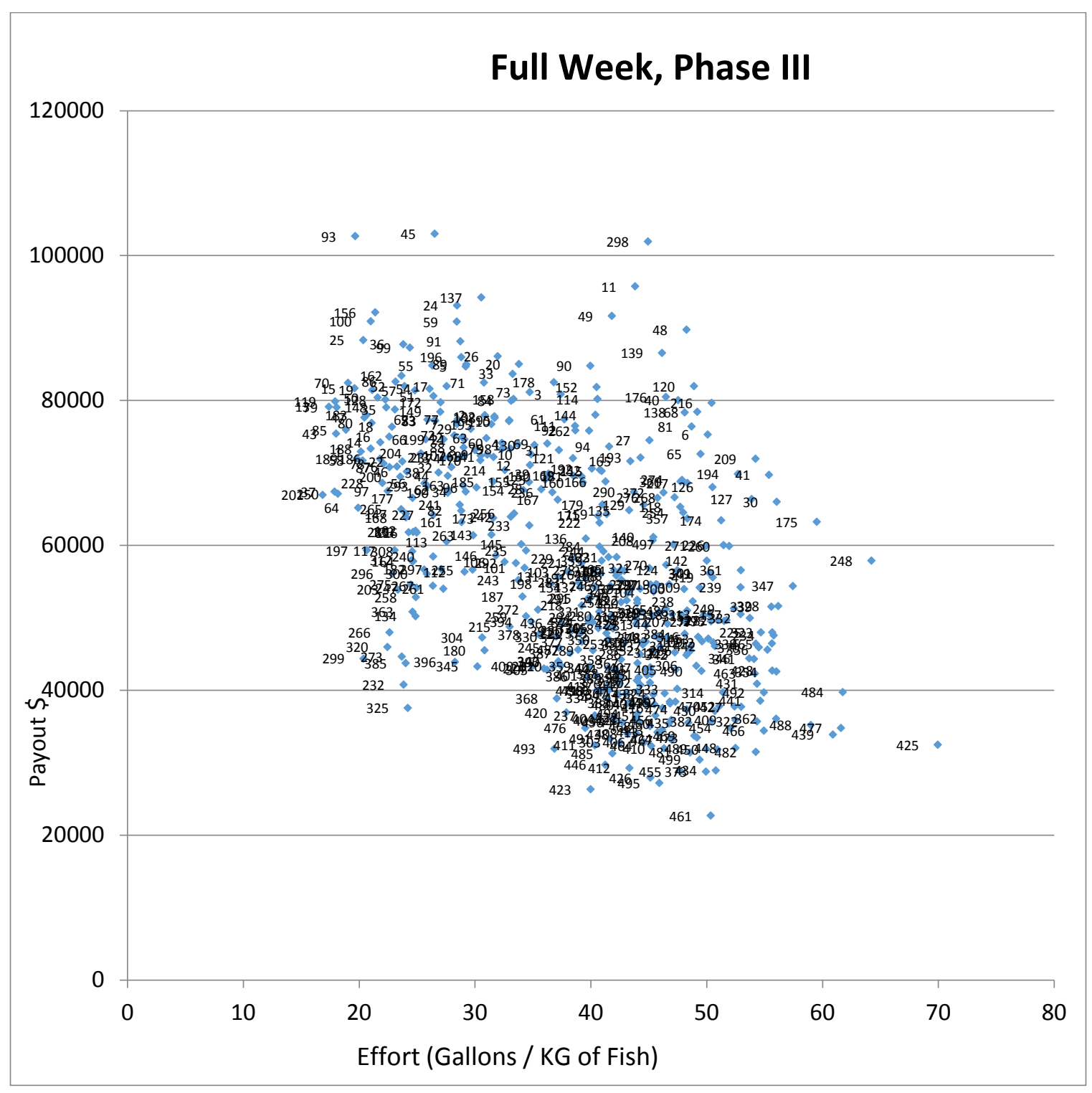

Figure 6.17: The Plot of 500 randomly generated tour values for Full Week in Phase III. 


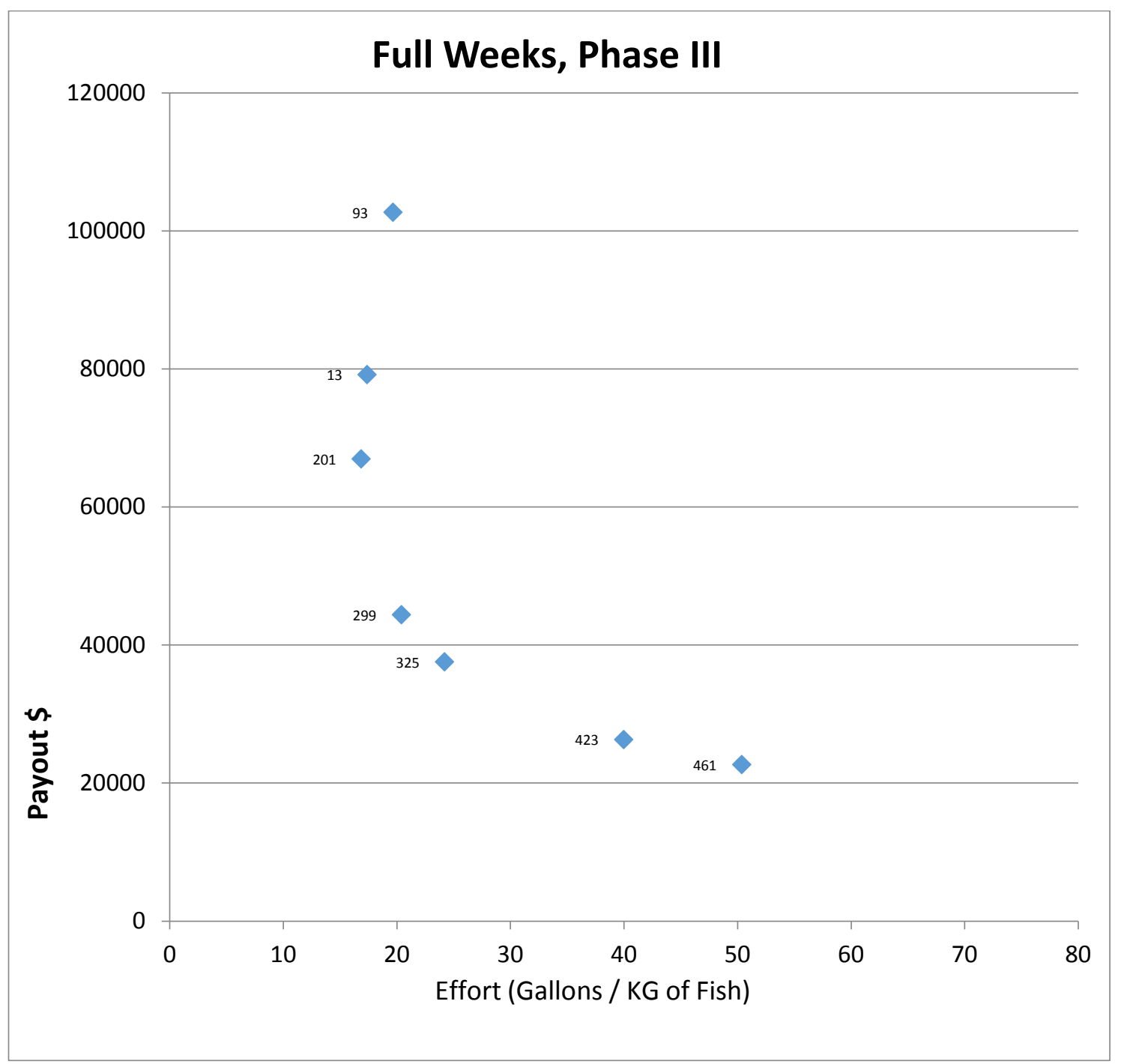

Figure 6.18: The dominant Pareto Front for Payout vs. Effort for Full week in Phase III. 


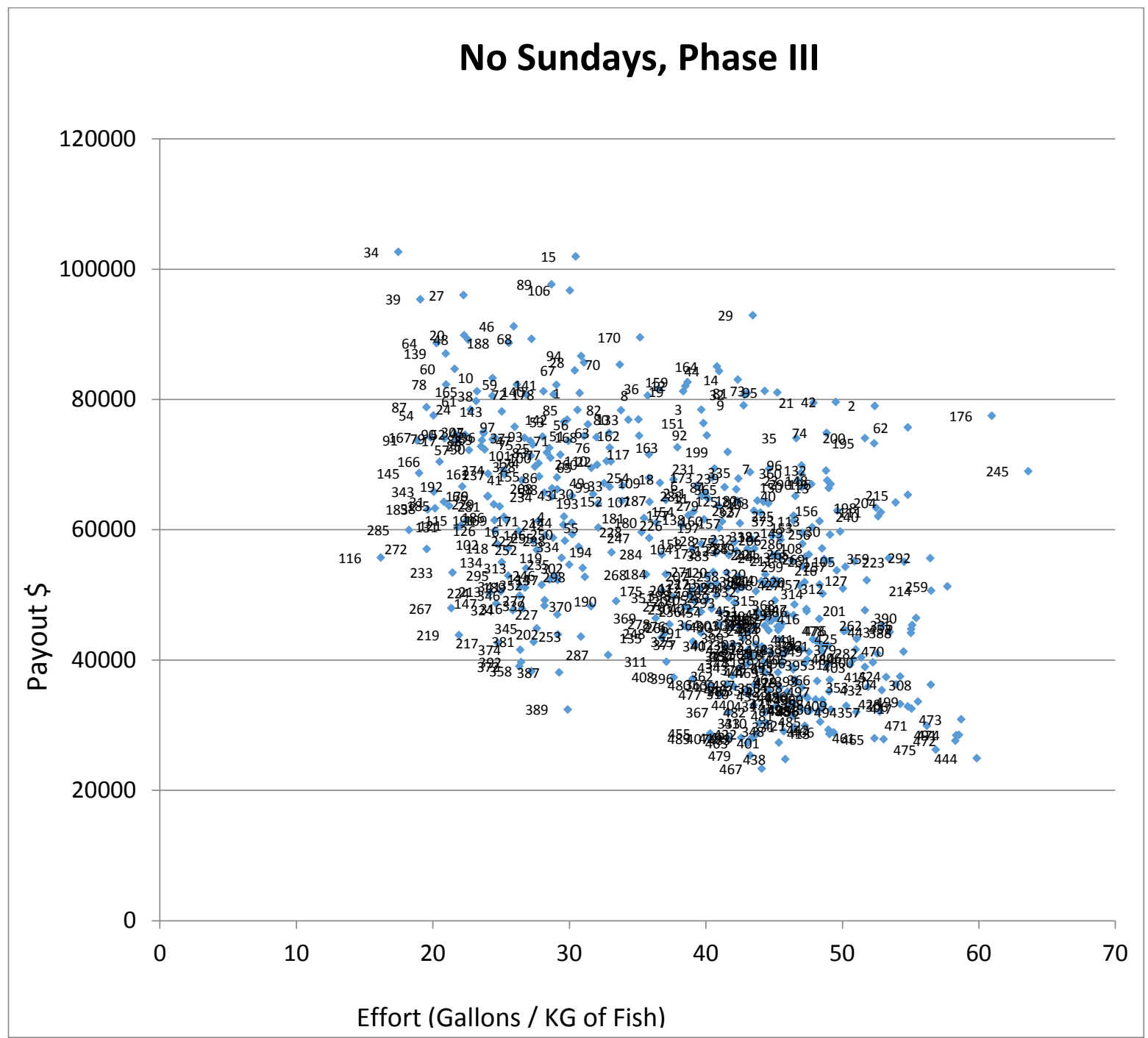

Figure 6.19: The Plot of 500 points of tour values that exclude Sundays in Phase III.

The distribution of tours that excluded Sundays is shown in Figure 6.19. Previously, the exclusion of Sundays worked to remove tours that fell in the middle range of the distribution. That is the same here but the excluded middle is much smaller than before. It appears that the uncertainty of the environment has been replaced by the more traditional one where not as many have the incentive to use Sundays as a buffer in order meet expectations in terms of Payout. In fact, the No Sundays Front is very much the same as that for the Full week in this Phase. 


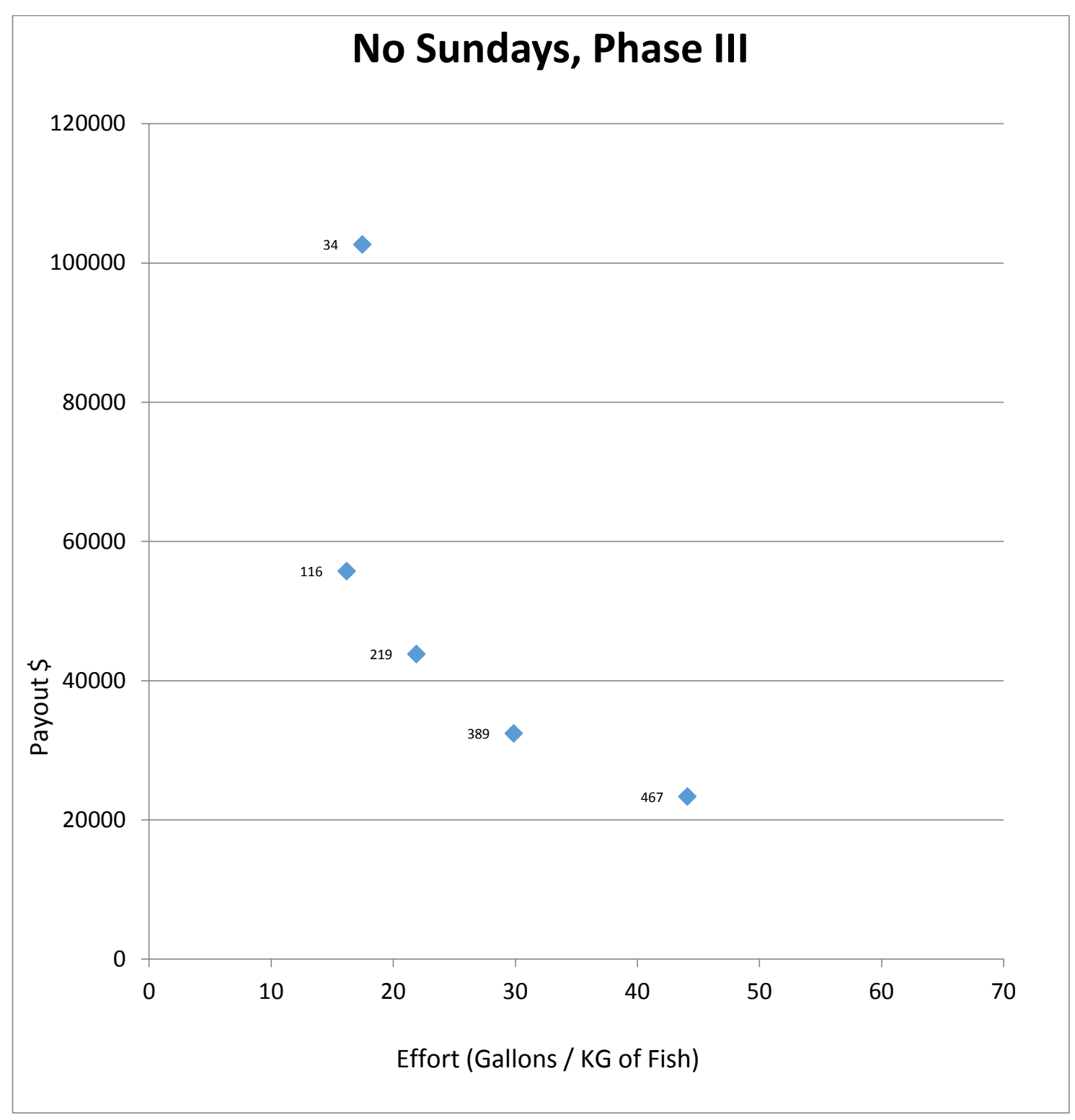

Figure 6.20: The Pareto Front for Payout vs. Effort for No Sundays. 


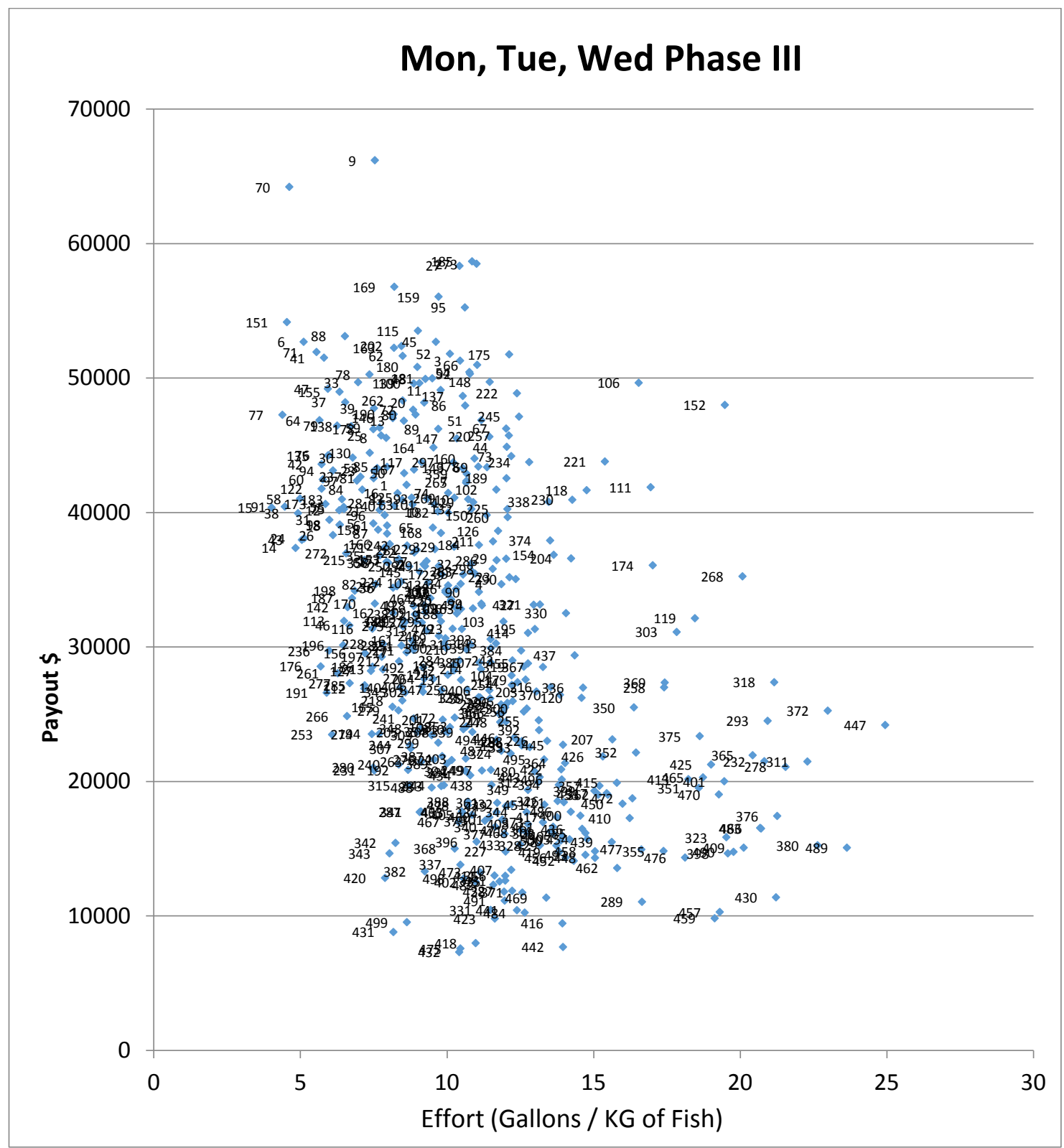

Figure 6.21: Plot of 500 randomly generated tours for MTW in Phase III.

Figure 6.21 displays the distribution of tours during the Phase that only took place in the first half of the week in the Back to Normal Phase. This tight cluster relates to the first portion of the Front with highest Payout. Clearly the strategy is focused more narrowly in and around Cerro Azul than in the previous La Nina phase. In fact the distribution stops around the inflection point of the No Sundays curve. This implies that those agents 
embarking on tours at the beginning of the week were able to successfully concentrate their search in and around Cerro Azul proper.

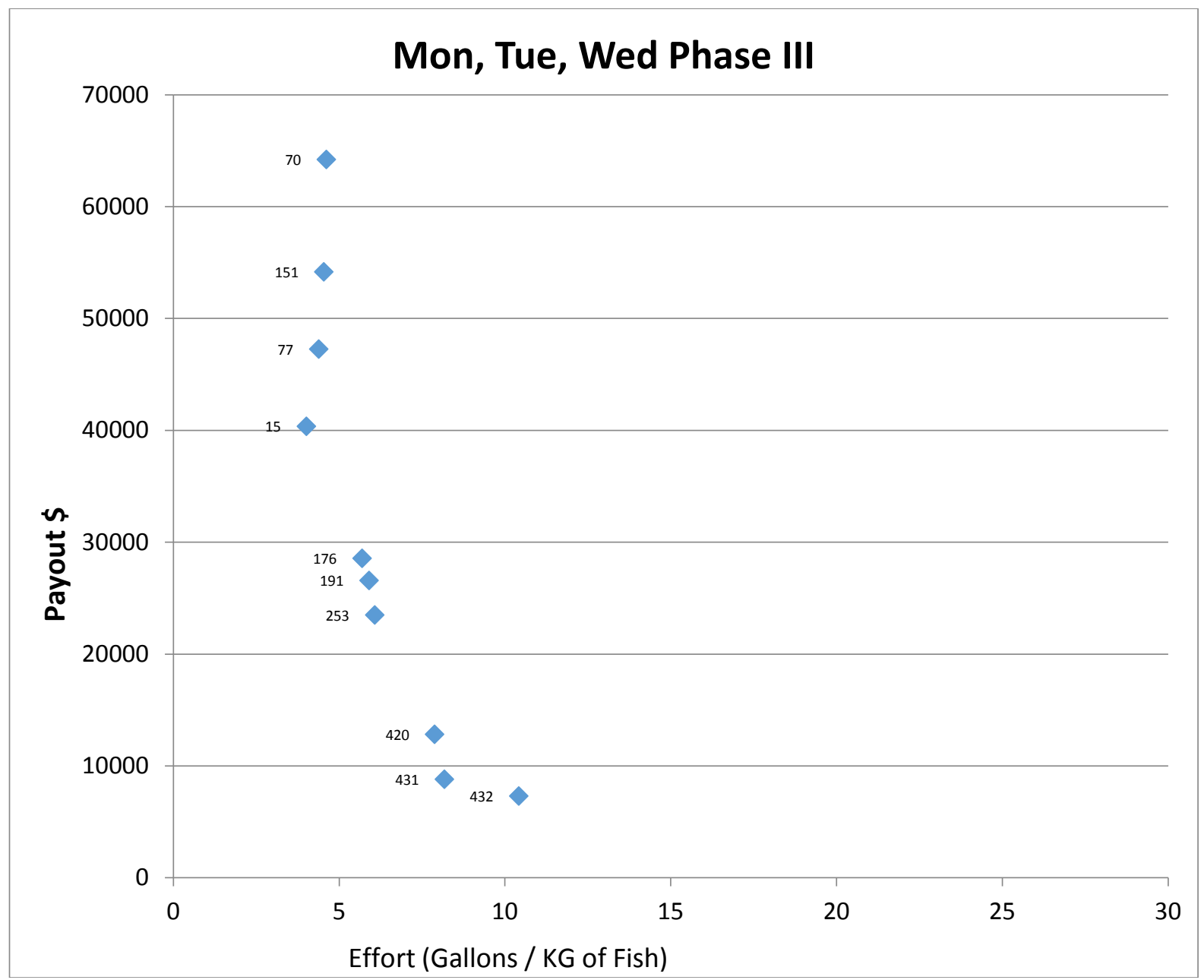

Figure 6.22: The Pareto Front for Payout vs. Effort for just MTW tours in Phase III.

Figure 6.22 shows the Pareto Front for the MTW tours and it exhibits a steep decline with the increased consumption of resources. Notice that it stops near the 10 unit barrier. This emphasizes the proclivity of the agents to stay as close to the areas that they know best now that things have returned to normal. 


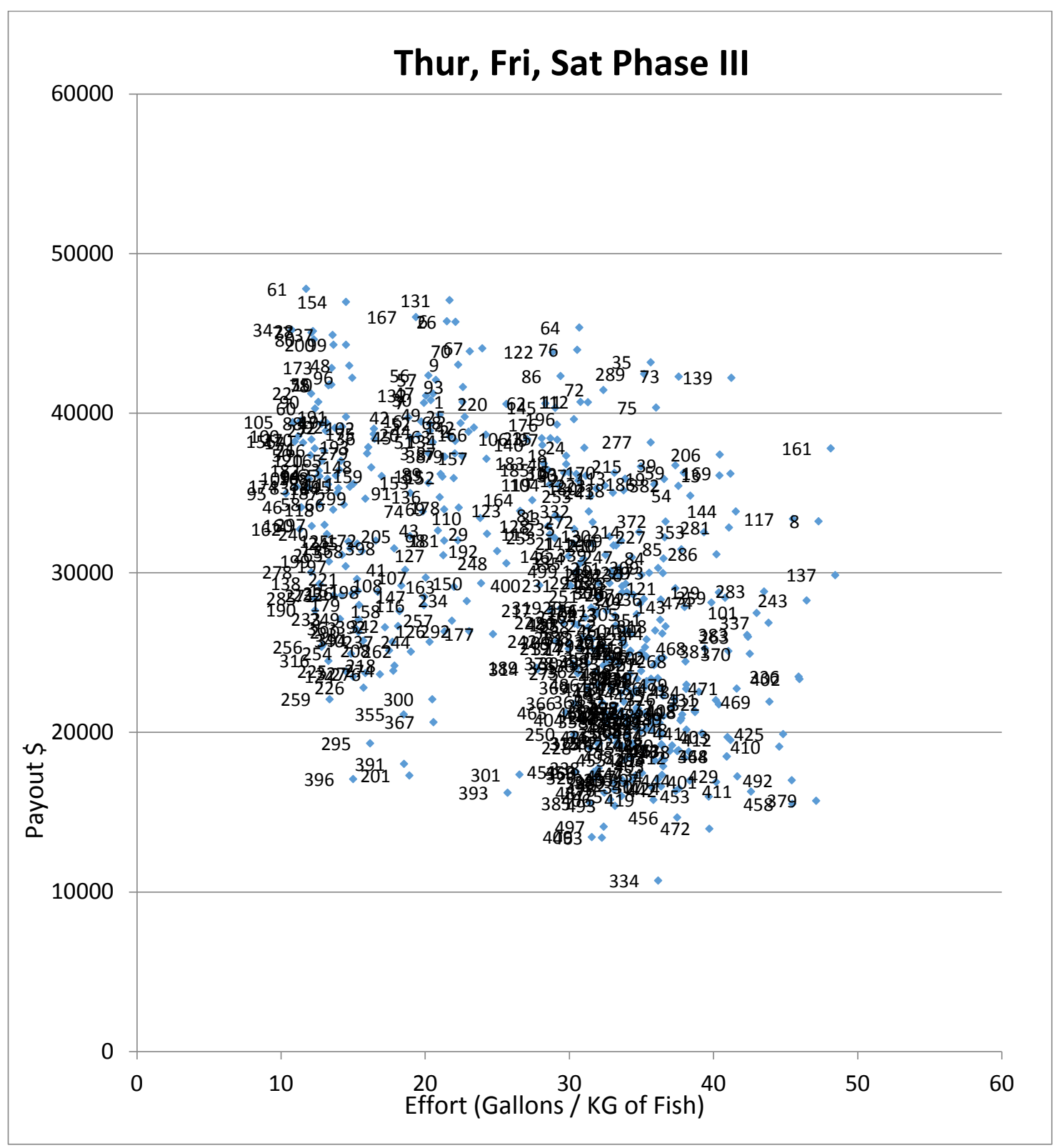

Figure 6.23: The Plot of 500 tour values for RFS tours in Phase III.

Figure 6.23 displays what might be viewed as three separate Pareto front, perhaps one for each of the three days. The bulk of the concentration requires a larger investment of resources than those with higher yields earlier in the front. A suggested interpretation is that the first front reflects a set of "mopping up" tours that are still able to find sufficient catches near Cerro Azul. The larger concentration may reflect the fact that tours that start on 
Thursday or Friday and go for longer distances may be returning on Saturday. In Figure 6.24 the first of the Pareto fronts is given, the one most likely to be associated with the mopping up of catch distribution near Cerro Azul. Notice the steep decline in productivity in response to added resources for the curve. It means that there will be less incentive for those later in the period to stay nearby and retrieve acceptable Payouts.

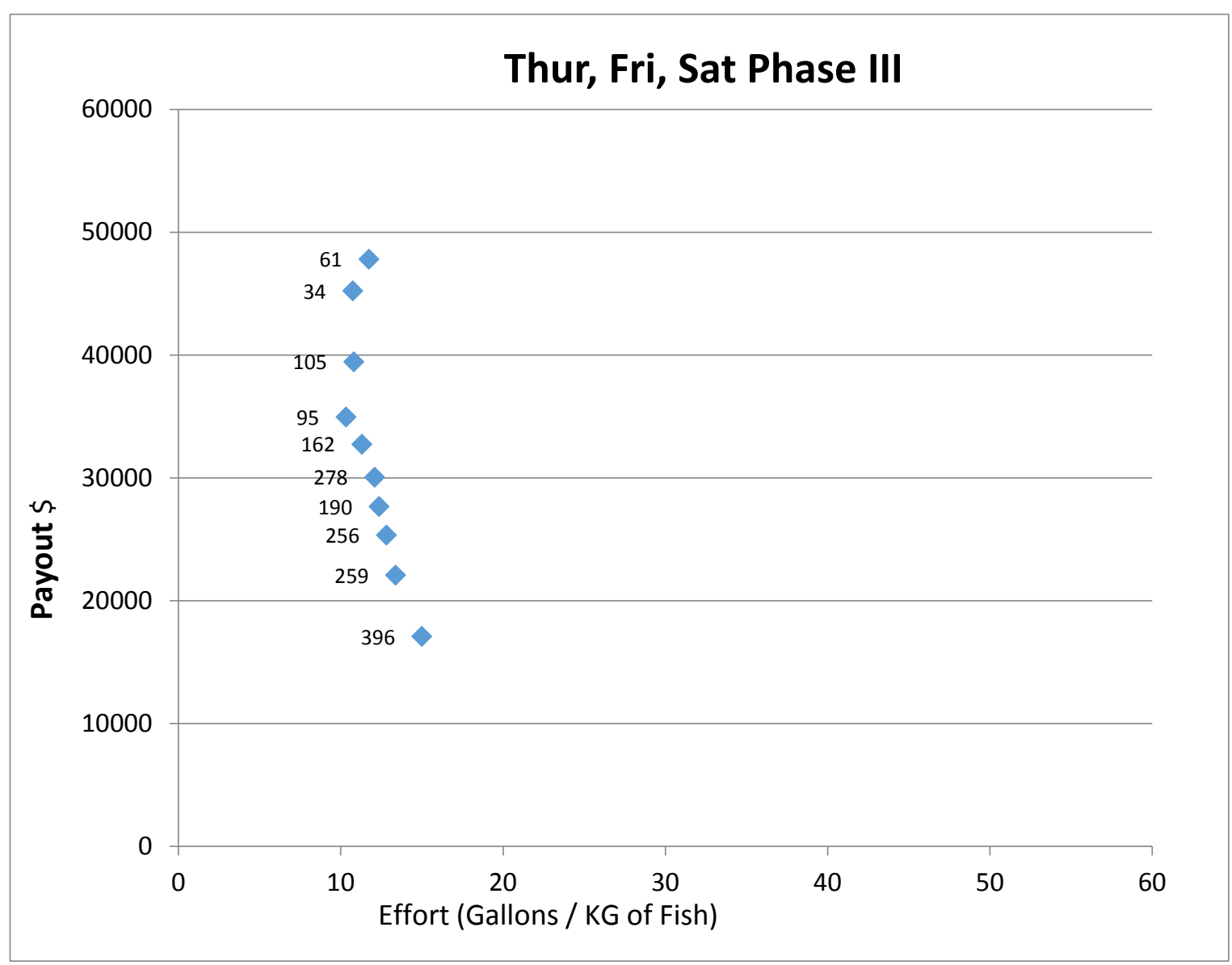

Figure 6.24: The Pareto Front for Payout vs. Effort in TFS Phase III.

\subsection{CONCLUSIONS}

Agent behavior was simulated relative to a number of tour constraints in each of the three ENSO phases under examination here. The results suggest that the nature of the curves reflected changes in the predictability of the environment and the increased use of resources 
to retain Payoff levels under more uncertain circumstances. This was particularly evident in the La Nina Phase where agents needed to invest more resource to achieve results similar to the Back to Normal phases. So agents handled the increased environmental variability with an increased emphasis on exploration and less on exploitation in their tours. When the system returned to a more state the agents were able to visibly consolidate their search to more wellknown areas than before and were able to expend less resources in doing so, this increased their profit margin by reducing the investment in buffering their trip planning with larger range search.

While these conclusions are somewhat qualitative in nature, we will provide statistical support for them in the subsequent chapter. There we will investigate whether the generated distribution of points for each of the scenarios can be viewed as an example of the same generation process, or whether the addition of tour constraints to the generation produced any significant differences in the results. In order to do this we will call upon the Cultural Algorithm Configuration described in Chapter 4 since that process is computationally expensive in nature [35]. 


\section{CHAPTER 7: STATISTICAL ANALYSIS OF THE MODEL PREDICTIONS FOR ALL PHASES}

We have developed curve fitting for the Artisanal Fishing problem using the Cultural Algorithm with Particle Swarm Optimization, CAPSO [35]. The fishing problem has two competing objective functions; the Payout $(\$)$ produced by a trip and the Effort required (Gallon $/ \mathrm{Kg}$ of fish). In the previous chapter we observed that the shape and structure of the computed Pareto fronts differed in shape and structure not only between the three different phases but within each phase based upon what days of the week a fishermen was constrained to follow. For example, what would the tours look like if the tour could take place on all days of the week, as opposed to those tours that did not consider Sundays as a day of departure and so on.

In this chapter we use statistical measures to test whether the distribution of tours produced by the Phase/Week combinations came from different theoretical distributions, in other words the choices available to fishermen. We will test the hypothesis that the values of the tours exhibit an overall non-linear distribution (hyper-linear) that is exhibited by the distribution of all tours and not just highlighted by the Pareto front. Another way to say this is whether the Pareto front expresses the overall trend of the resultant tours instead of just being representative of a small segment of tours. The result of the analysis is to suggest that our bi-objective model is indeed an accurate way to characterize the patterns of results found in the database.

To this end we present twelve scenarios, three phases with four depart day cases. The four depart day cases are the allowable days of the week to go out fishing: 1) Full week; 2) No Sundays (full week without Sundays); 3) Mon_Tue_Wed - Only three days $1^{\text {st }}$ half of the 
week Monday's, Tuesday's and Wednesday's; finally, 4) Thur_Fri_Sat $2^{\text {nd }}$ half of the week Thursday's, Friday's and Saturday's. A Pareto frontier using Hyperbolic Regression curve fitting compared to Linear Regression lines and the original points are shown for all cases. The statistical results derived from testing the equivalence between the hyper-linear equation and the linear one is done for all 12 scenarios.

\subsection{ANALYSIS OF THE RESIDUAL TESTING}

There are two ways to test how well the Pareto Frontier line fits the data: by residual plot and by coefficient of determination (the squared correlation, $\mathrm{r})^{2}$. The squared correlation, $r^{2}$, gives the fraction of the data's variance that is accounted for by our bi-objective model. Thus, $\left(1-r^{2}\right)$ is the fraction of the original variance left in the residuals that is left unexplained by the patterns produced by our model. $r^{2}$ is always between $0 \%$ and $100 \%$. When $r^{2}$ is equal to 0 , then the data are unrelated. However, when the sum of squared residuals equals the total sum of squares for $\mathrm{y}$ that leads to the best linear predictor for $\mathrm{y}$ is which just the mean of $y$ itself. The Residual is the difference between the observed value of response the payout (Y) and the predicted (Yhat). The residuals test will reveal how well our fishing model works.

\subsection{COMPARISON OF CURVE FITTING AND RESIDUAL PHASE I}

We present four cases for phase I- El Nino. The four cases have tours of different duration for the REGULAR fishermen as follows: 1. Full week; 2. No Sundays (full week without Sundays); 3. Only three days $1^{\text {st }}$ half of the week Monday's, Tuesday's and Wednesday's; finally, 4. $2^{\text {nd }}$ half of the week Thursday's, Friday's and Saturday's. All scenarios conducted for High Desirability (HD) with most relative effort (MRE). 
A Pareto frontier using Hyperbolic Regression curve produced by CAPSO is compared to Linear Regression lines and the original points for the residuals are depicted for all cases. Figure 7.1 provides the Curve fitting for Payout [\$] Versus Effort [Gallon $/ \mathrm{Kg}$ of fish], for Full week Phase I with High Desirability. Figure 7.2 gives the scatterplot points for the Residuals for Full week Phase I with High Desirability. Figure 7.3 shows the Curve fitting for Payout (\$) Versus Effort (Gallon/Kg of fish), for No Sundays Phase I with High Desirability. Figure 7.4 exhibits the scatterplot points for the Residuals for No Sundays Phase I with High Desirability. Figure 7.5 shows Curve fitting for Payout (\$) Versus Effort (Gallon $/ \mathrm{Kg}$ of fish), for $1^{\text {st }}$ half week Monday's, Tuesday's and Wednesday's Phase I with High Desirability. Figure 7.6 has the scatterplot of points for the Residuals for $1^{\text {st }}$ half week Monday's, Tuesday's and Wednesday's for Phase I with High Desirability. Figure 7.7 gives the Curve fitting for Payout (\$) Versus Effort (Gallon $/ \mathrm{Kg}$ of fish), for $2^{\text {nd }}$ half week, Thursday's, Friday's and Saturday's for Phase I with High Desirability. Figure 7.8 shows the scatterplot of points for the Residuals for $2^{\text {nd }}$ half week, Thursday's, Friday's and Saturday's for Phase I with High Desirability.

An examination of the residuals, the differences between the predictions for the CAPSO produced hyper-linear model and the best fit linear one suggest that the values, although relatively uniform across effort, are large enough to suggest that the two model's predictions are statistically different. In general, the hyperlinear model for All days and Non Sundays tends to over-predict with low effort and under-predict with higher effort. For the Mon_Tue_Wed and Thur_Fri_Sat graphs the residuals are more closely aligned. However, in these cases the non-linear model under-predicts with lower effort and over predicts when effort is higher. They tend to cancel each other out so as to reduce the overall sums in each. 
This suggests that the first two scenarios will be rejected and the last two rejected for the null hypothesis that the distribution of model tours is linear in nature.

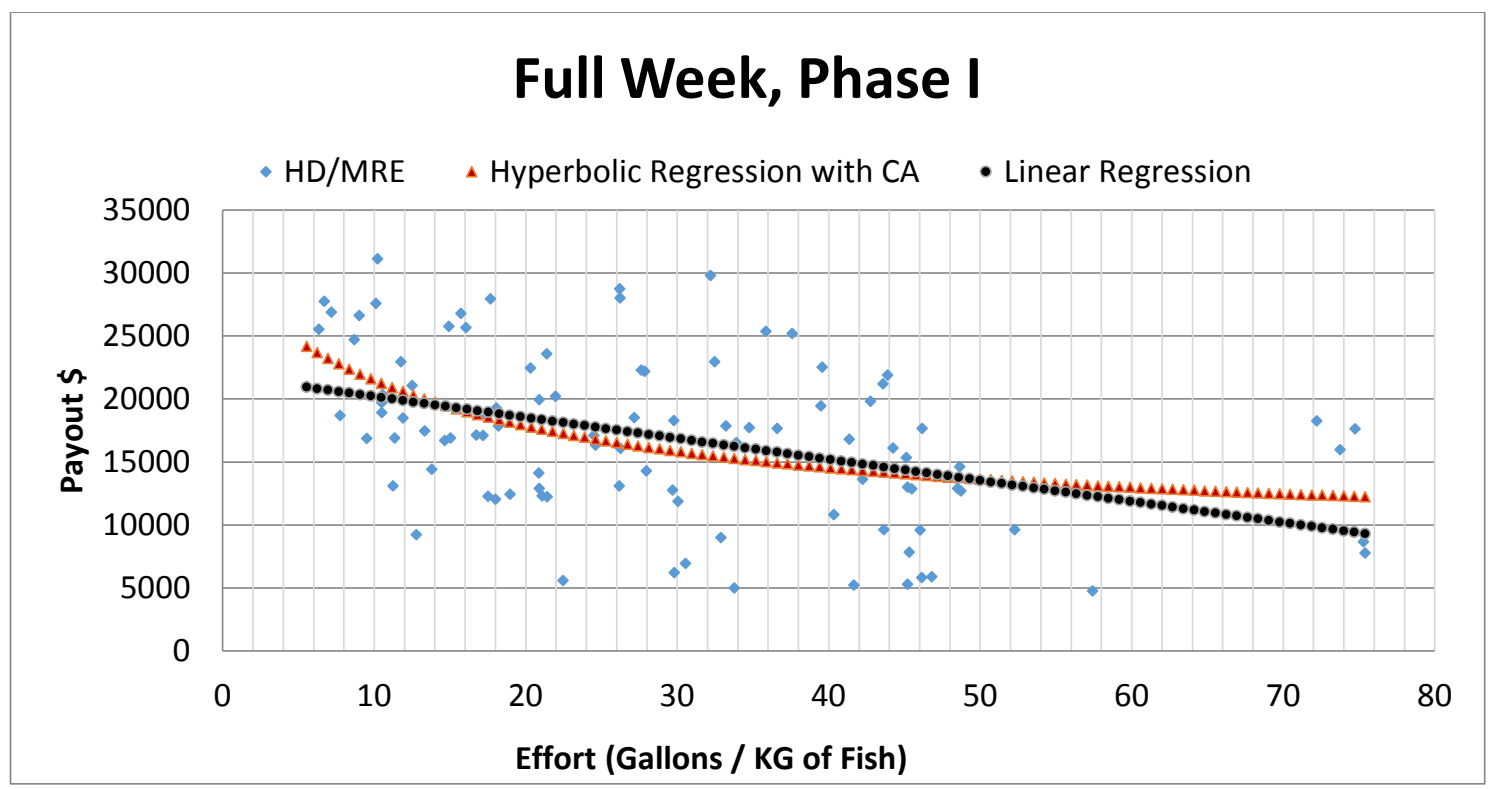

Figure 7.1: Curve fitting for Payout, Effort for Full week Phase I.

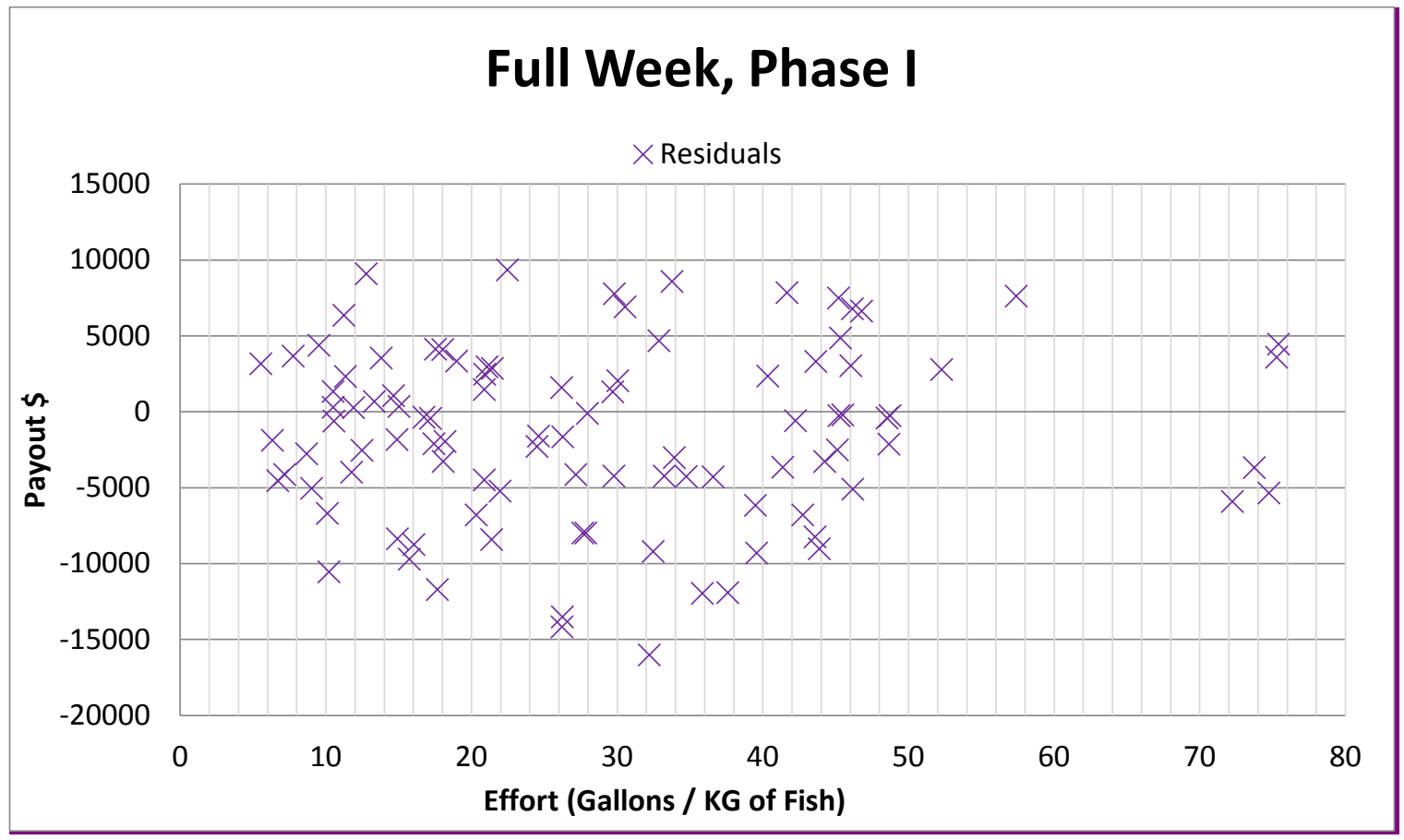

Figure 7.2: Scattering points for Residuals for Full week Phase I. 


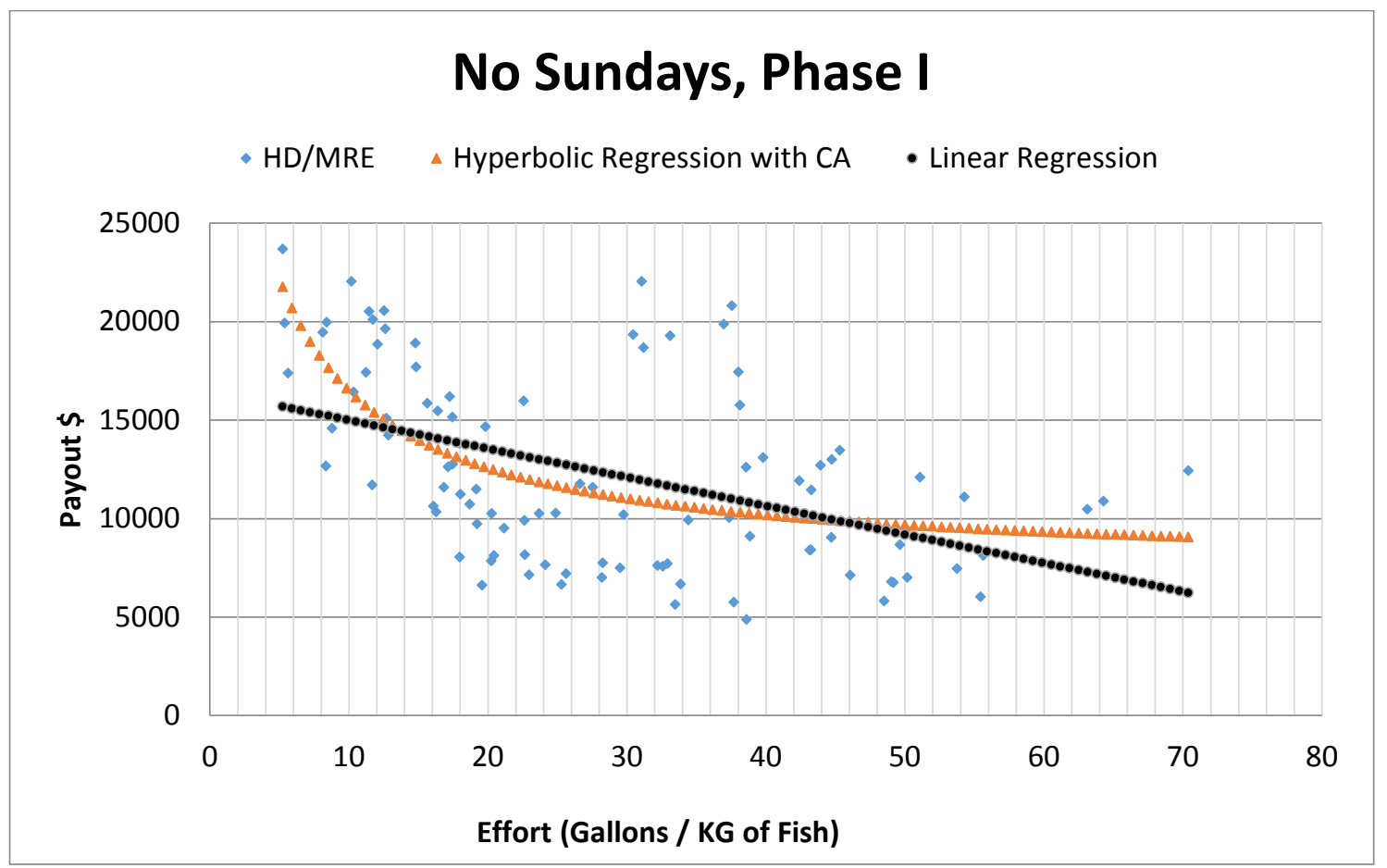

Figure 7.3: Curve fitting for Payout, Effort for No Sundays Phase I.

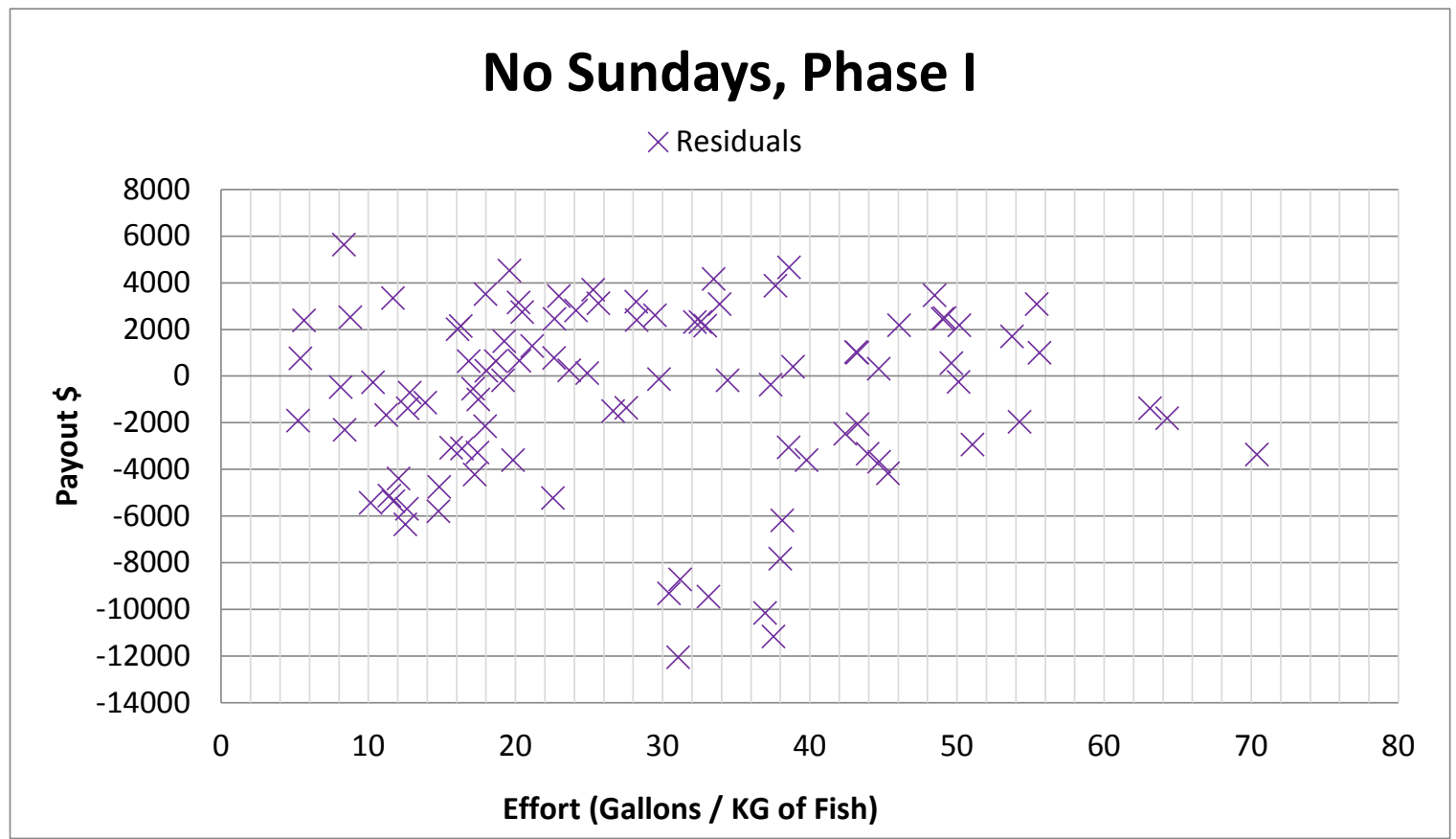

Figure 7.4: Scattering points for Residuals for No Sundays Phase I 


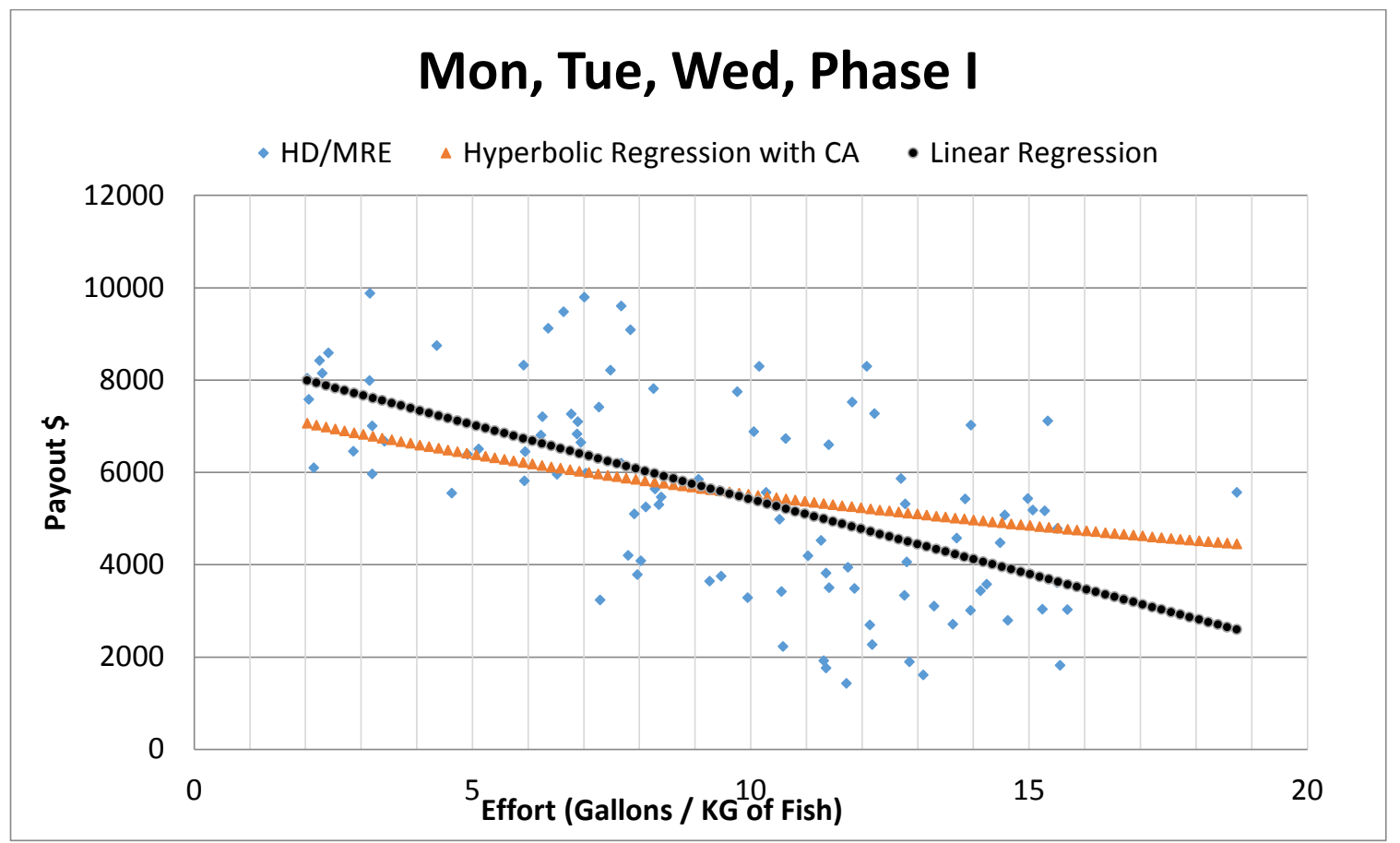

Figure 7.5: Curve fitting for Payout, Effort for $1^{\text {st }}$ half of the week Phase I

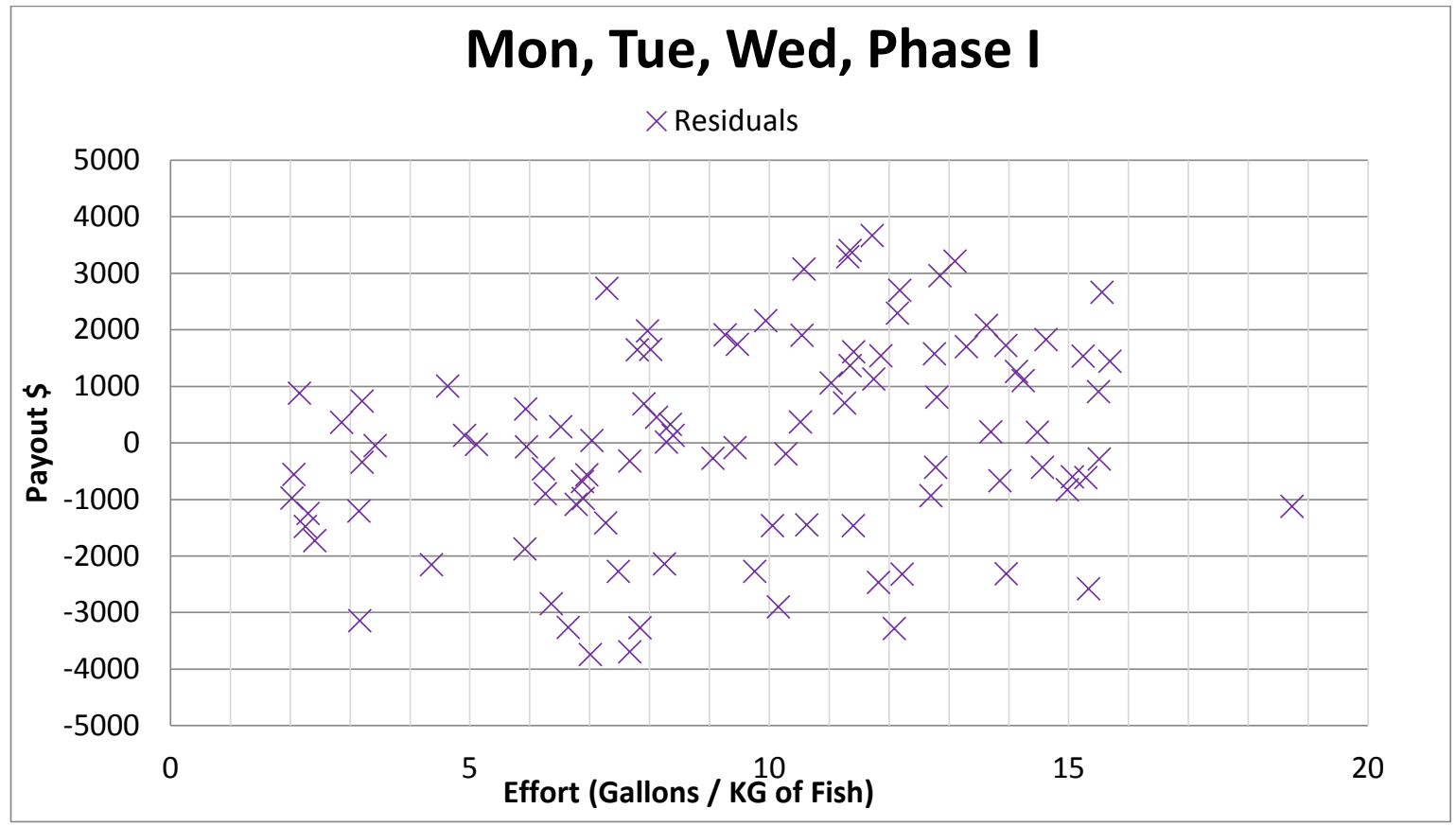

Figure 7.6: Scattering points for Residuals, $1^{\text {st }}$ half of the week Phase I 


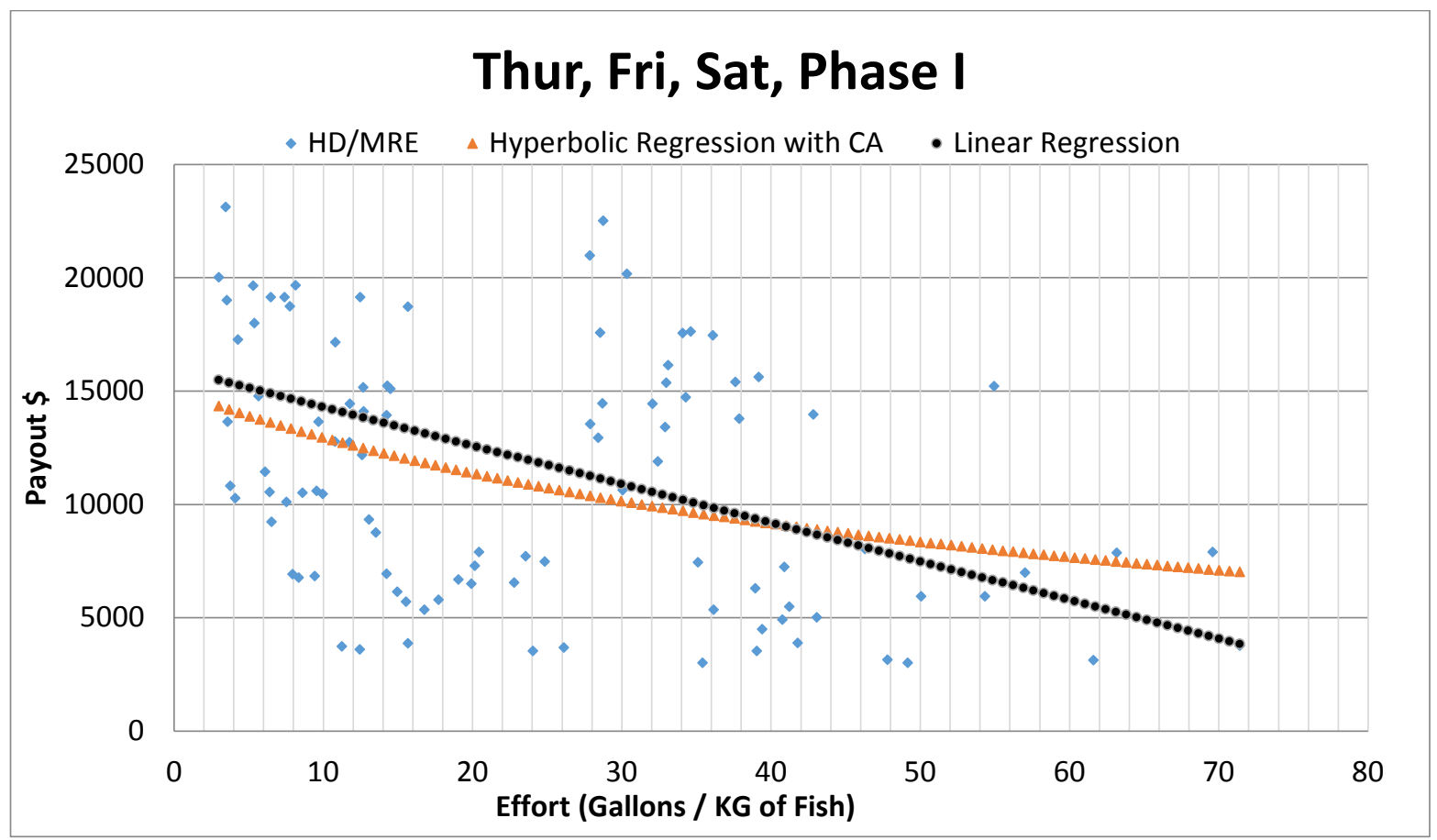

Figure 7.7: Curve fitting for Payout, Effort, $2^{\text {nd }}$ half of the week Phase I

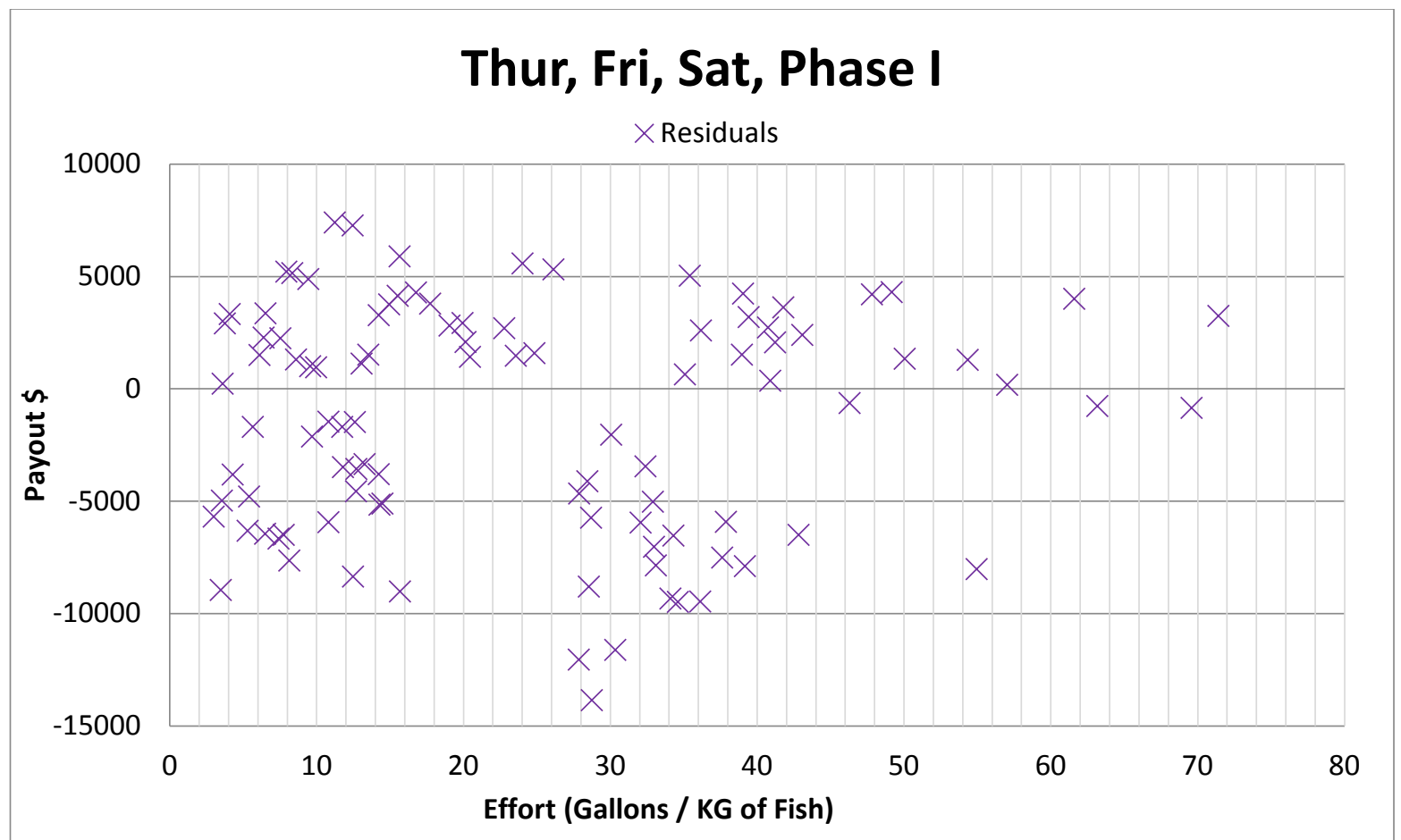

Figure 7.8: Scattering points for Residuals, $2^{\text {nd }}$ half of the week Phase I 


\subsection{COMPARISON OF HYPER-LINEAR AND LINEAR MODEL PREDICTIONS IN PHASE II}

In this section we present four cases for phase II- La Nina. The four cases have different trip durations for the fishermen as follows; 1. Full week; 2. No Sundays (full week without Sundays); 3. Only three days $1^{\text {st }}$ half of the week Monday's, Tuesday's and Wednesday's; finally, 4. $2^{\text {nd }}$ half of the week Thursday's, Friday's and Saturday's. A Pareto frontier using Hyperbolic Regression curve fitting compared to Linear Regression lines and the original points for the residuals are depicted for all cases. Figure 7.9 shows Curve fitting for Payout [\$] Versus Effort [Gallon / $\mathrm{Kg}$ of fish], for Full week Phase II with High Desirability. Figure 7.10 shows the scattering points for the Residuals for Full week Phase II with High Desirability. Figure 7.11 shows Curve fitting for Payout [\$] Versus Effort [Gallon $/ \mathrm{Kg}$ of fish], for No Sundays Phase II with High Desirability. Figure 7.12 shows the scattering points for the Residuals for No Sundays Phase II with High Desirability. Figure 7.13 Shows Curve fitting for Payout [\$] Versus Effort [Gallon $/ \mathrm{Kg}$ of fish], for $1^{\text {st }}$ half week Monday's, Tuesday’s and Wednesday's Phase II with High Desirability. Figure 7.14 Shows the scattering points for the Residuals for $1^{\text {st }}$ half week Monday's, Tuesday's and Wednesday's for Phase II with High Desirability. Figure 7.15 shows Curve fitting for Payout [\$] Versus Effort [Gallon /Kg of fish], for $2^{\text {nd }}$ half week, Thursday's, Friday's and Saturday’s for Phase II with High Desirability. Figure 7.16 Shows the scattering points for the Residuals for $2^{\text {nd }}$ half week, Thursday's, Friday's and Saturday’s for Phase II with High Desirability.

The residual plot of the difference between the predictions of the hyperlinear model and the best fit linear one are uniformly large and in some cases tend to increase with effort. The non-linear model in all cases tends to over-predict in all cases with low effort and over 
predict slightly with large effort. This suggests that the tests for significance based upon the residuals will likely result in a rejection of the null hypothesis in each.

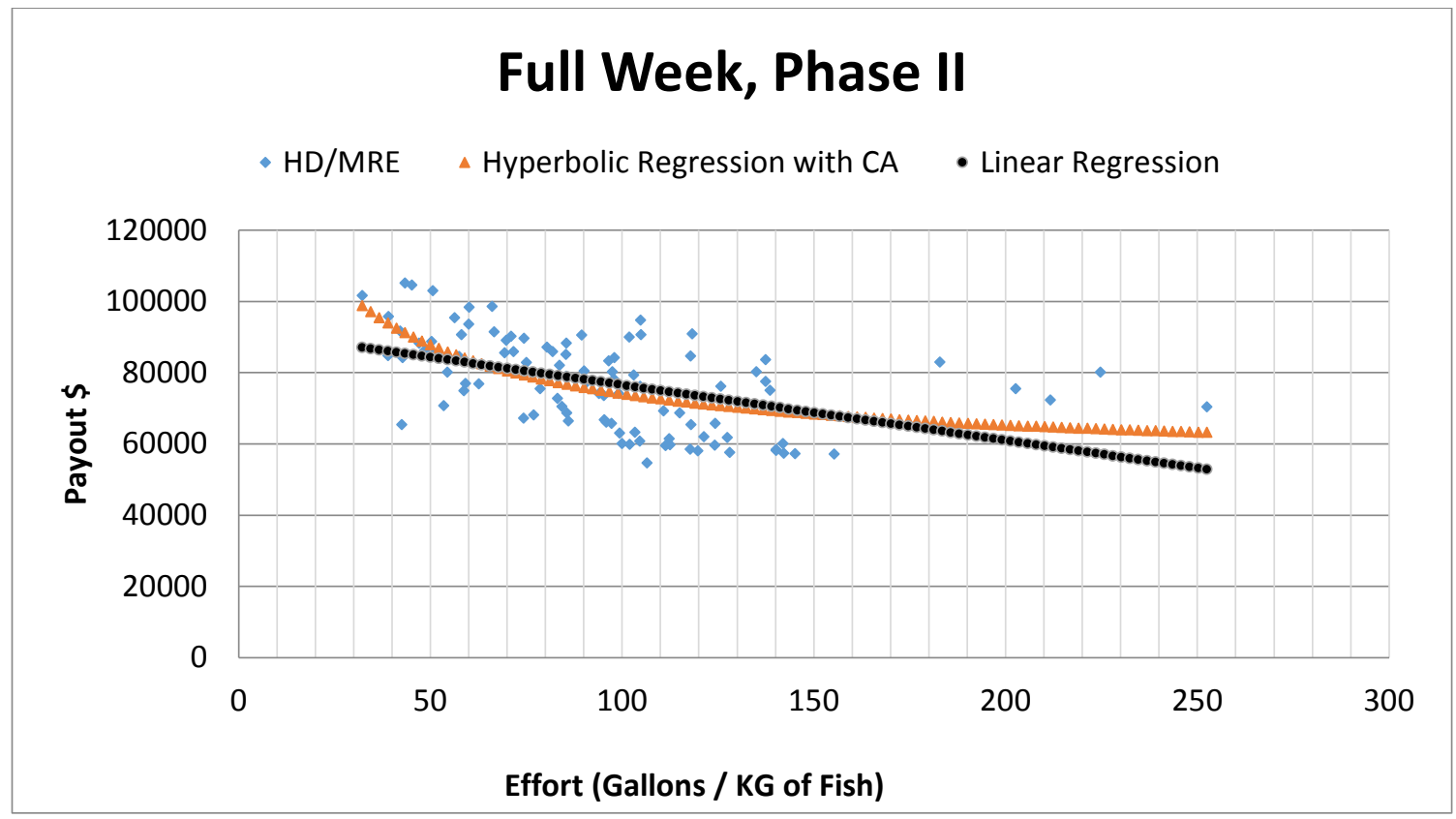

Figure 7.9: Curve fitting for Payout, Effort, for Full Week Phase II.

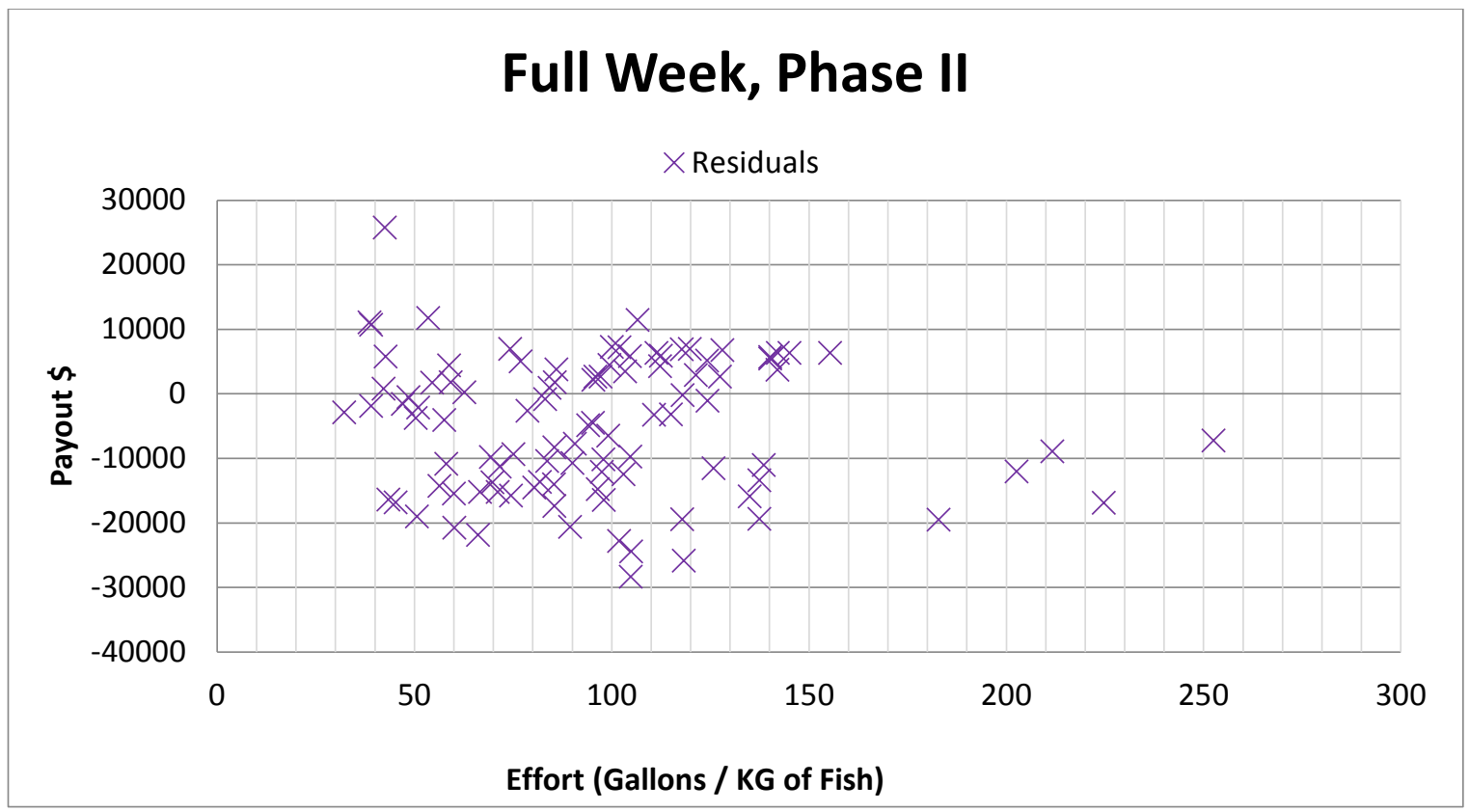

Figure 7.10: Scattering points for Residuals for Full week Phase II 


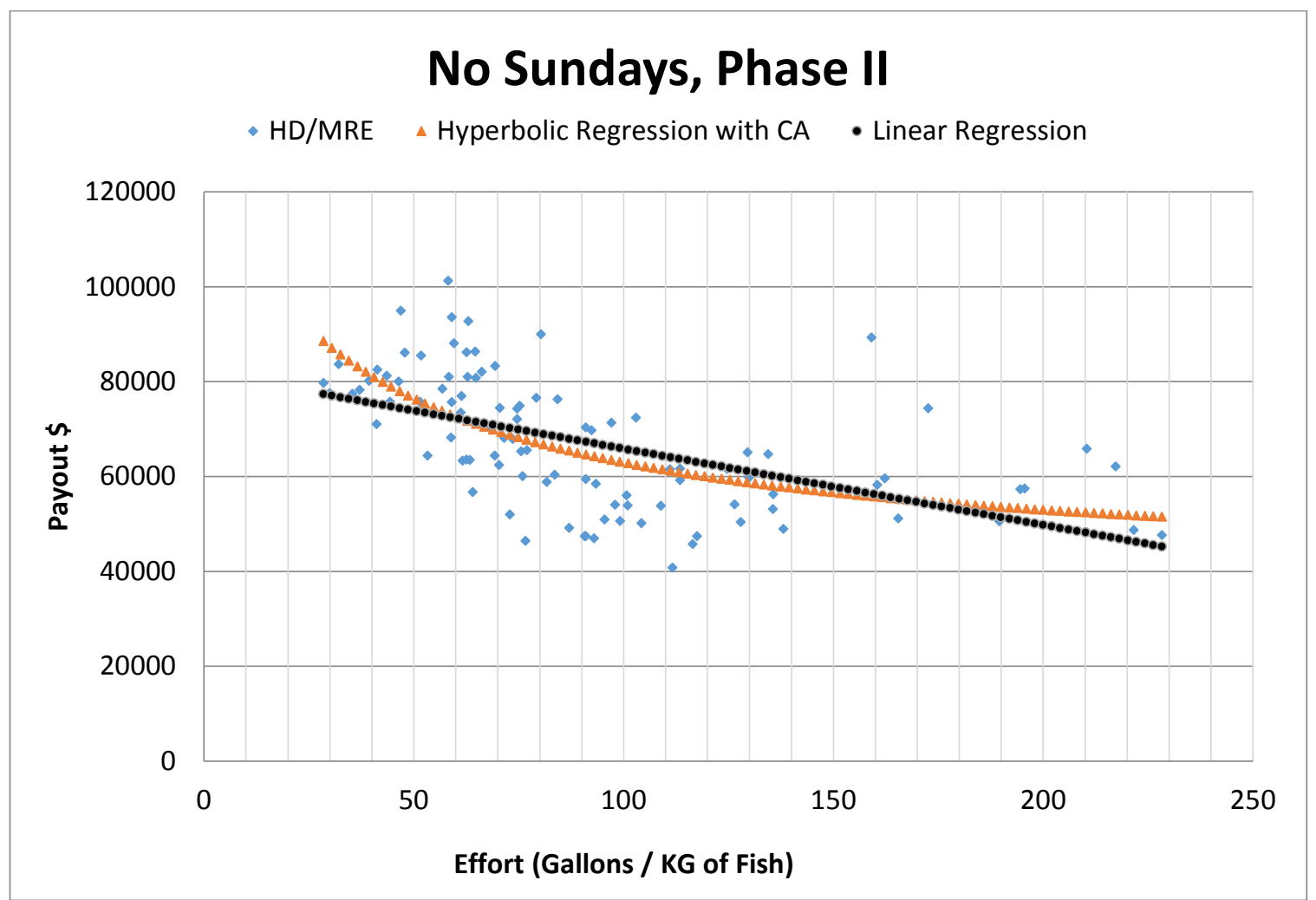

Figure 7.11: Curve fitting for Payout, Effort for No Sundays Phase II

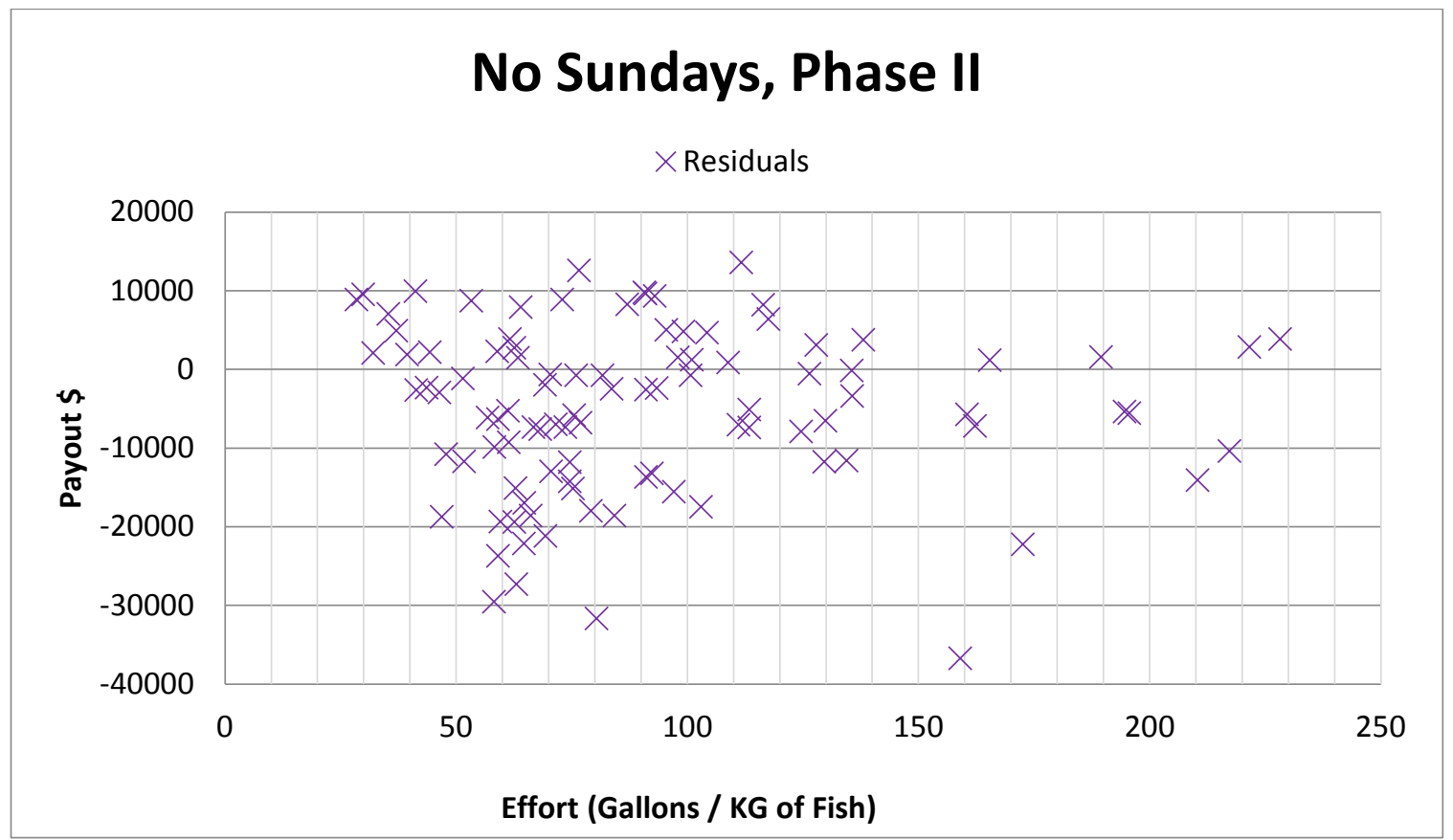

Figure 7.12: Scattering points for Residuals for No Sundays Phase I 


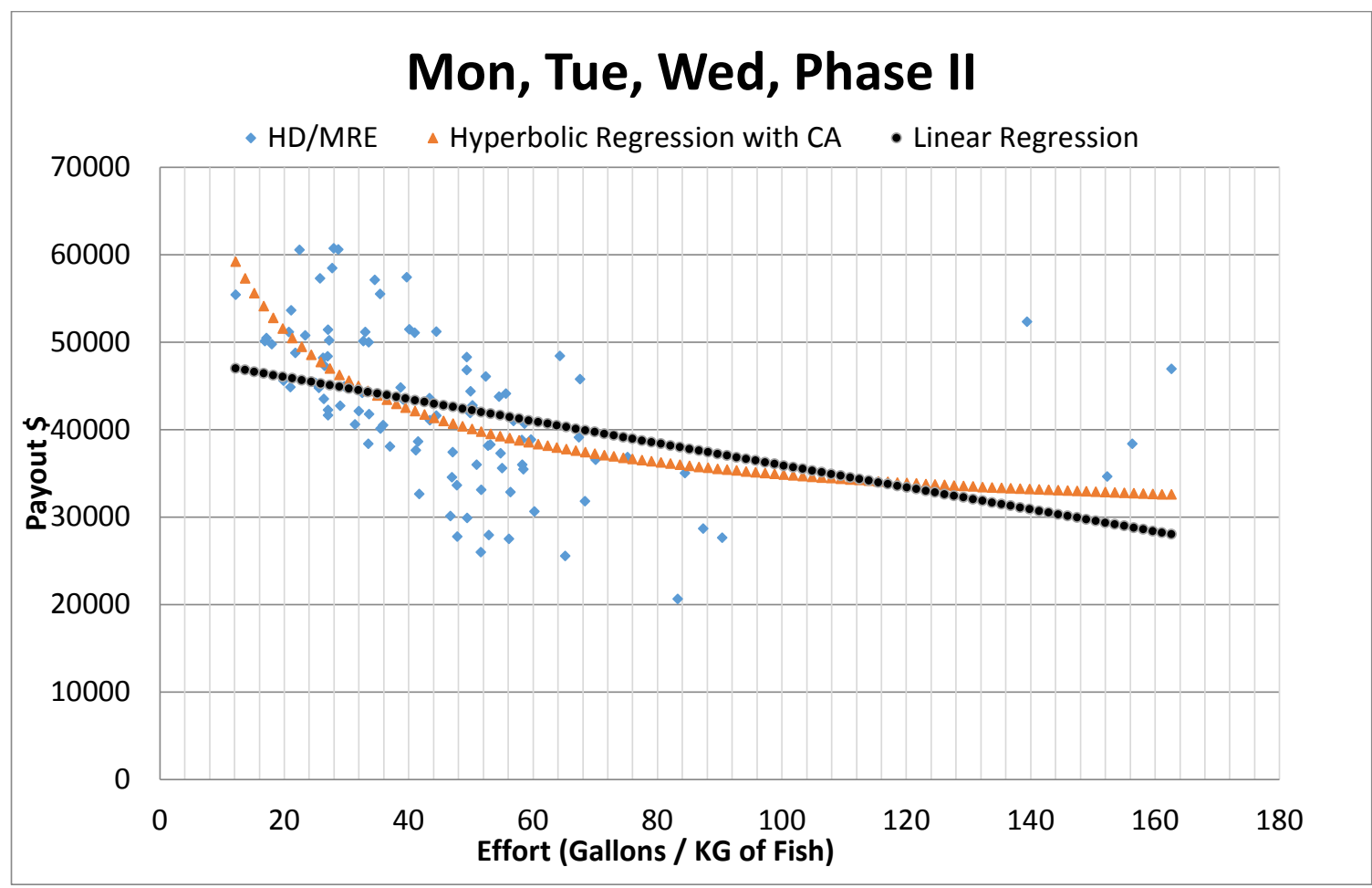

Figure 7.13: Curve fitting for Payout, Effort for MTW Phase II

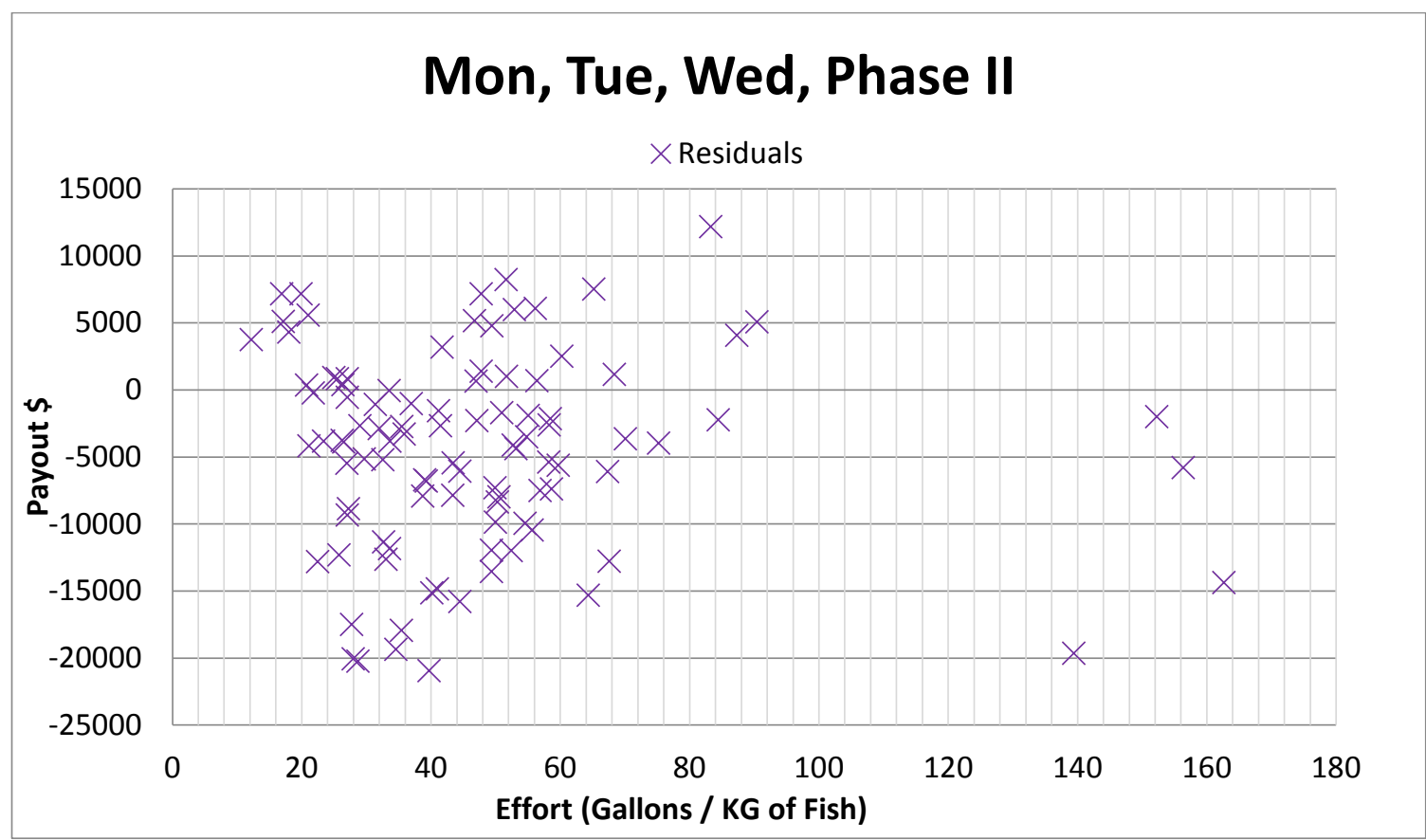

Figure 7.14: Scattering points for Residuals for MTW Phase II 


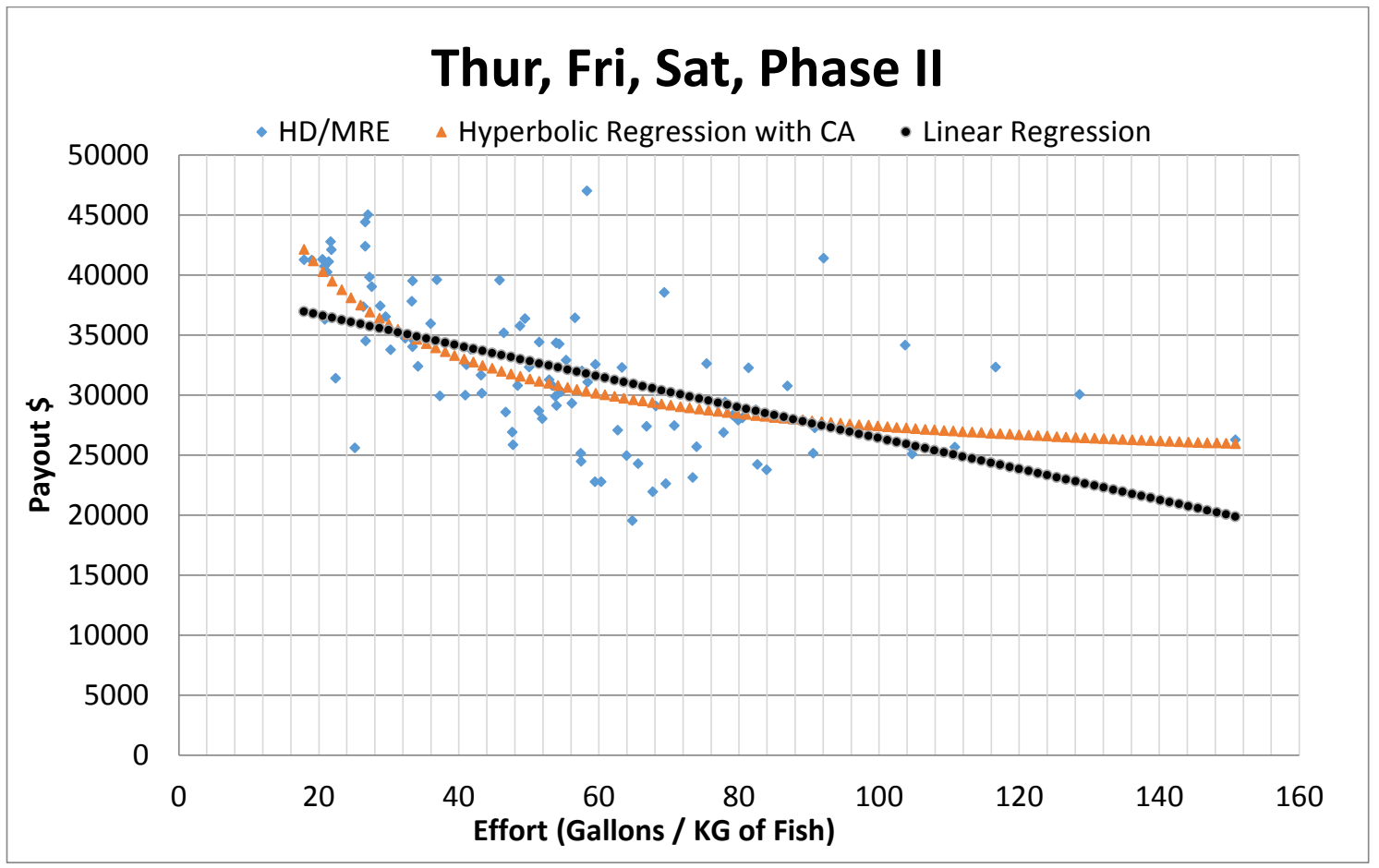

Figure 7.15: Curve fitting for Payout, Effort for TFS Phase II.

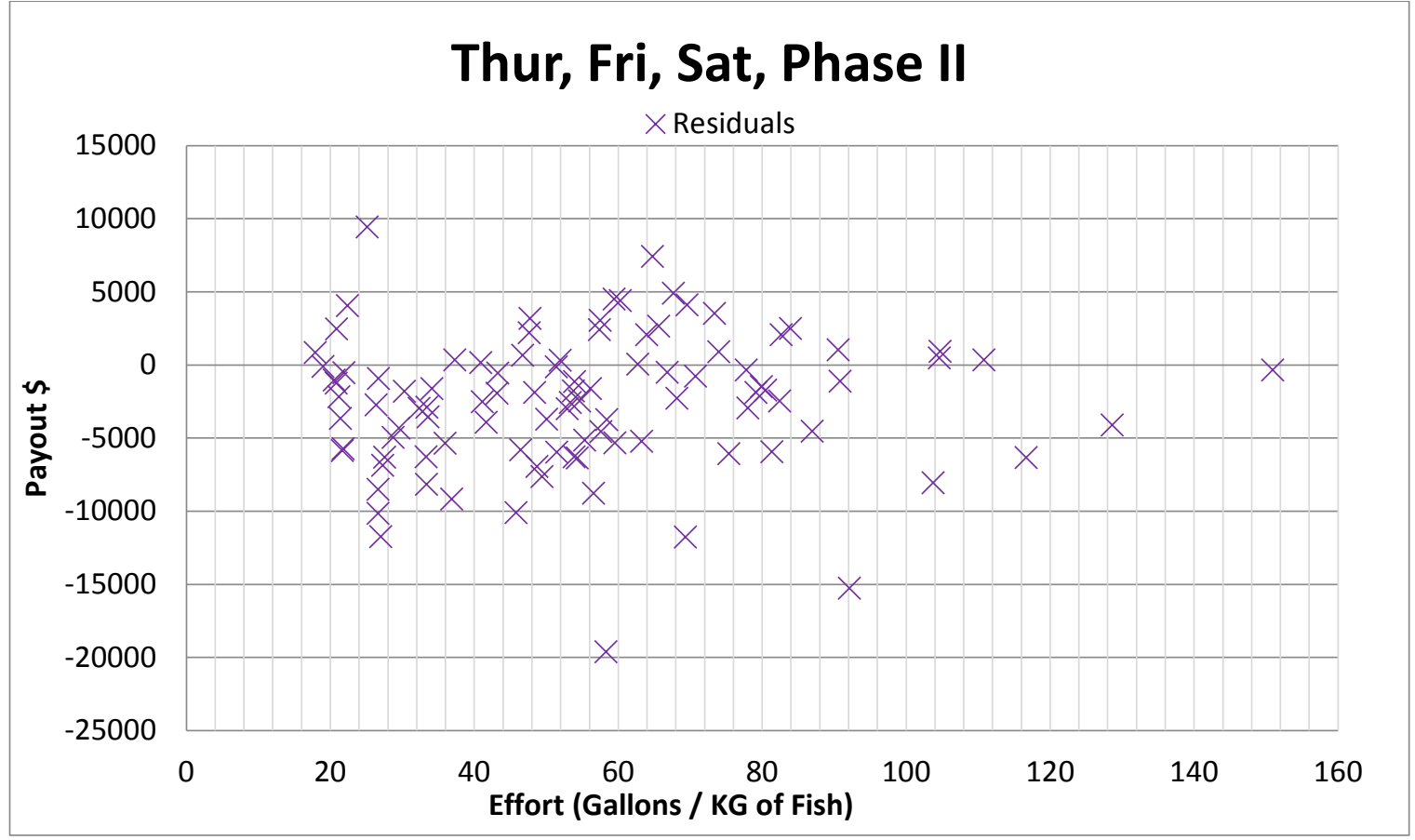

Figure 7.16: Scattering points for Residuals for TFS Phase II 


\subsection{COMPARISON OF HYPERLINEAR CURVES AND RESIDUALS IN PHASE III}

We present four cases for phase III- back to normal. The four cases have different constraints on days for fishing as before: 1. Full week; 2. No Sundays (full week without Sundays); 3 . Only three days $1^{\text {st }}$ half of the week Monday's, Tuesday's and Wednesday's; finally, 4. $2^{\text {nd }}$ half of the week Thursday's, Friday's and Saturday's. All scenarios are conducted for High Desirability with Most Relative Effort.

A Pareto frontier using Hyperbolic Regression curve fitting is compared to Linear Regression lines and the original points for the residuals are depicted for all cases. Figure 7.17 shows Curve fitting for Payout [\$] Versus Effort [Gallon /Kg of fish], for Full week Phase III with High Desirability. Figure 7.18 gives the scattering points for the Residuals for Full week Phase III with High Desirability. Figure 7.19 gives Curve fitting for Payout [\$] Versus Effort [Gallon /Kg of fish], for No Sundays Phase III with High Desirability. Figure 7.20 shows the scattering points for the Residuals for No Sundays Phase III with High Desirability. Figure 7.21 shows Curve fitting for Payout [\$] Versus Effort [Gallon /Kg of fish], for $1^{\text {st }}$ half week Monday's, Tuesday's and Wednesday's Phase III with High Desirability. Figure 7.22 shows the scattering points for the Residuals for $1^{\text {st }}$ half week Monday's, Tuesday's and Wednesday's for Phase III with High Desirability. Figure 7.23 shows Curve fitting for Payout (\$) Versus Effort (Gallon $/ \mathrm{Kg}$ of fish), for $2^{\text {nd }}$ half week, Thursday's, Friday's and Saturday's for Phase III with High Desirability. Figure 7.24 shows the scattering points for the Residuals for $2^{\text {nd }}$ half week, Thursday's, Friday's and Saturday's for Phase III with High Desirability.

The residuals plots exhibit the differences in the predictions between the best fit linear and hyperlinear models respectively. The size of the residuals in all cases is relatively 
large and in the cases of Mon_Tue_Wed and Thur_Fri_Sat the residuals increase with increased effort. This suggests that the hyper-linear model is better at predicting behavior nearer to Cerro Azul than farther away in these situations. This is interesting since it indicates that now the system is back to normal, and the agents are better able to predict what is going on in the areas that they are familiar with. 


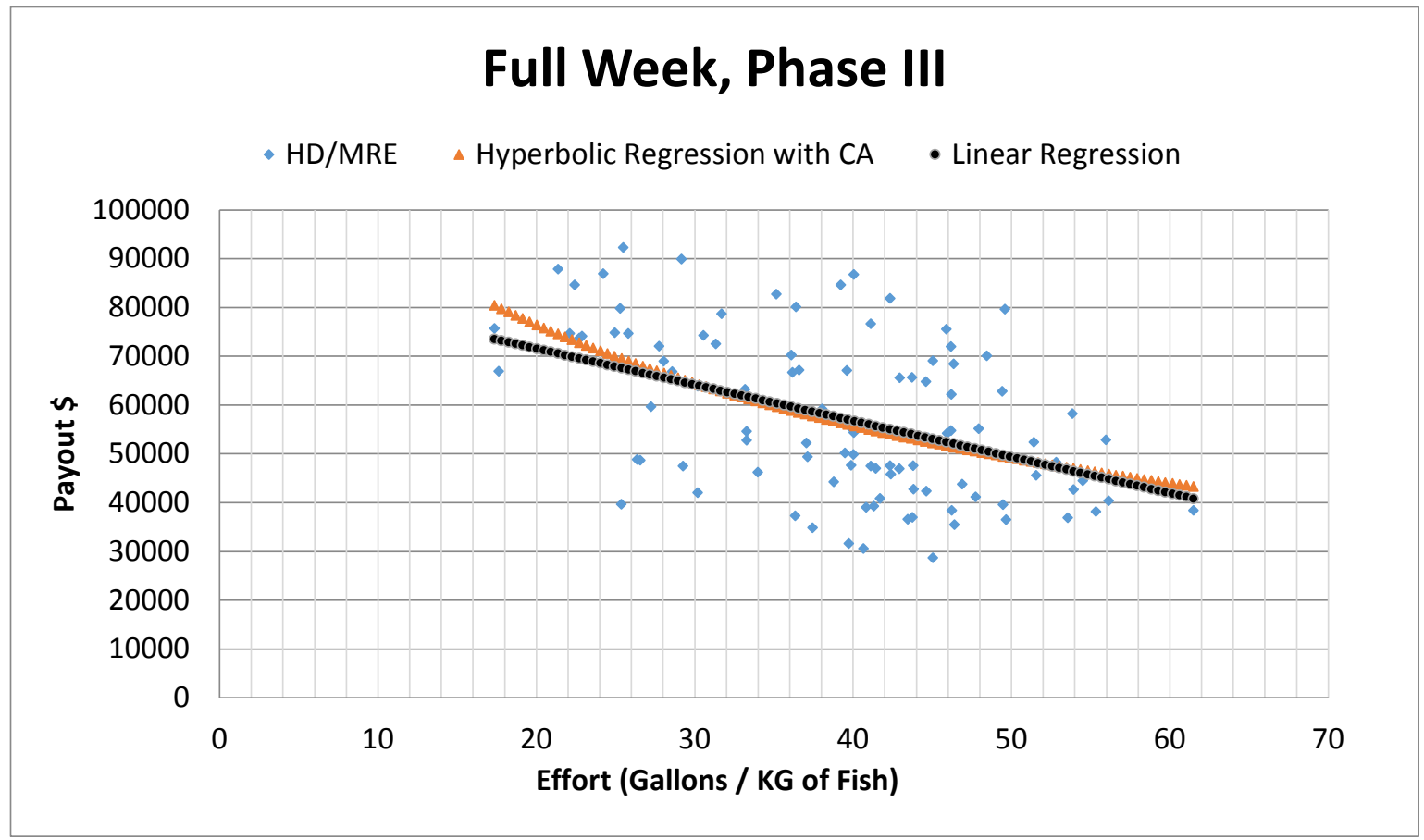

Figure 7.17: Curve fitting for Payout, Effort for Full week Phase III.

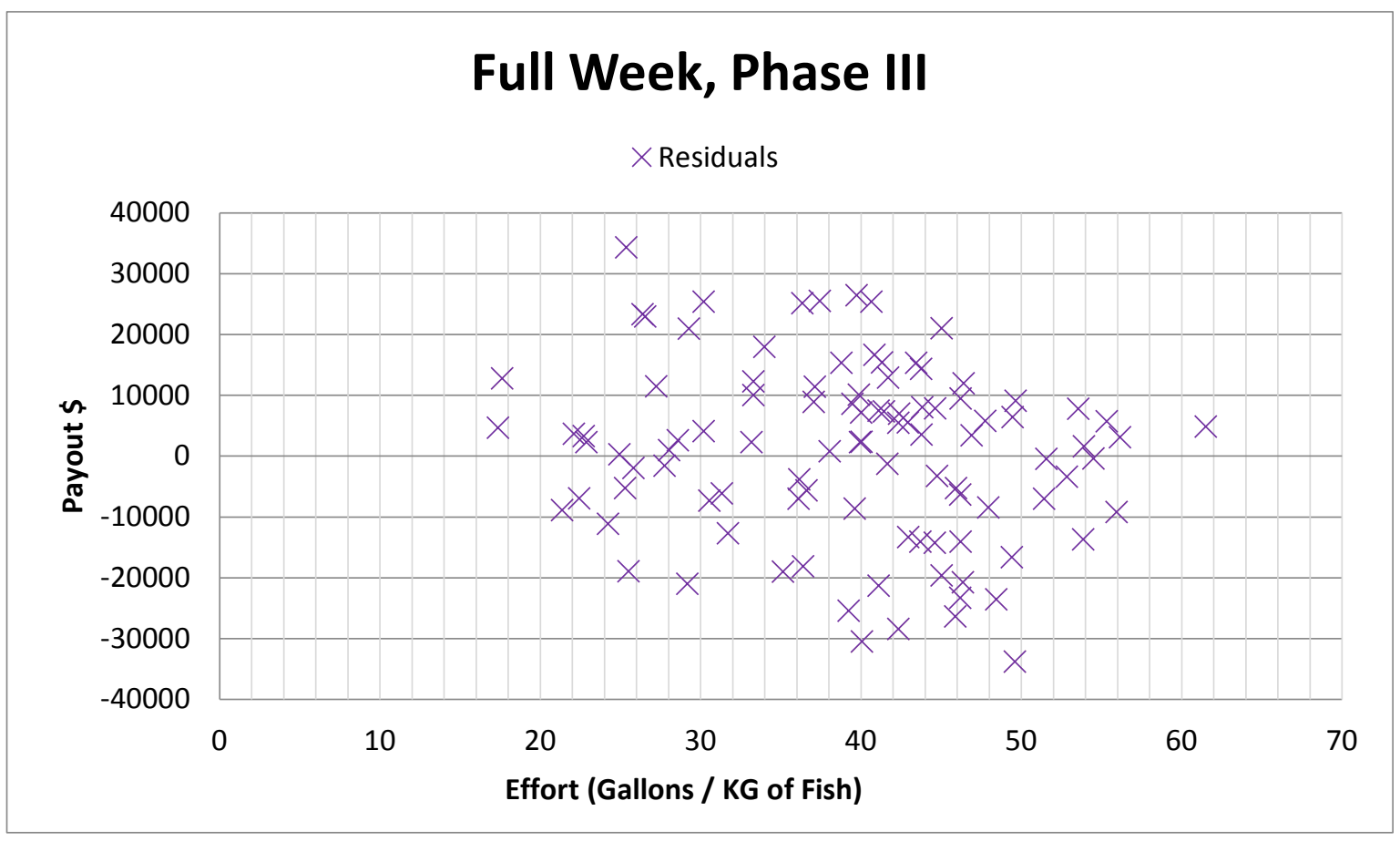

Figure 7.18: Scattering points for Residuals for Full Week for Phase III 


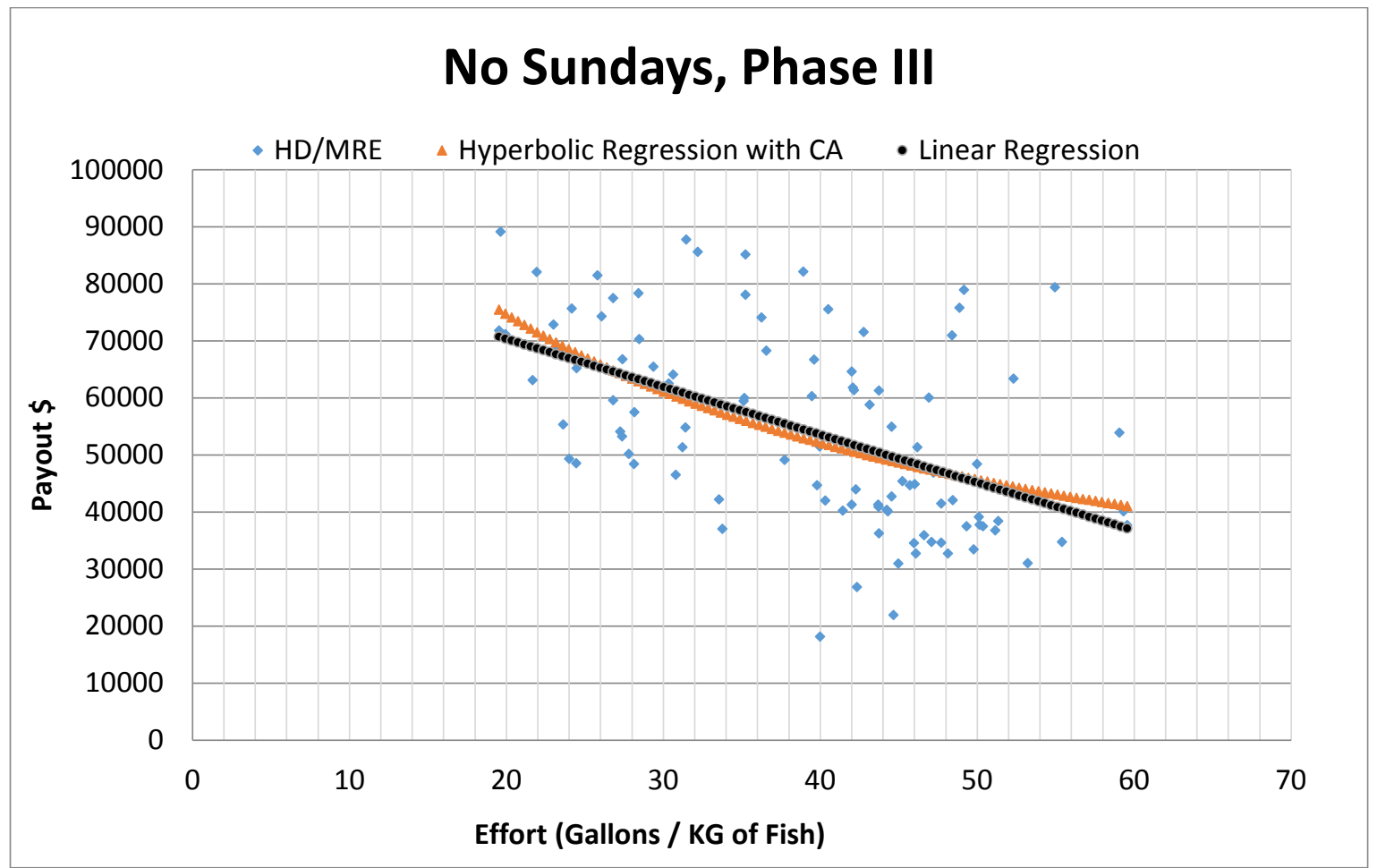

Figure 7.19 Curve fitting for Payout, Effort for NS Phase III.

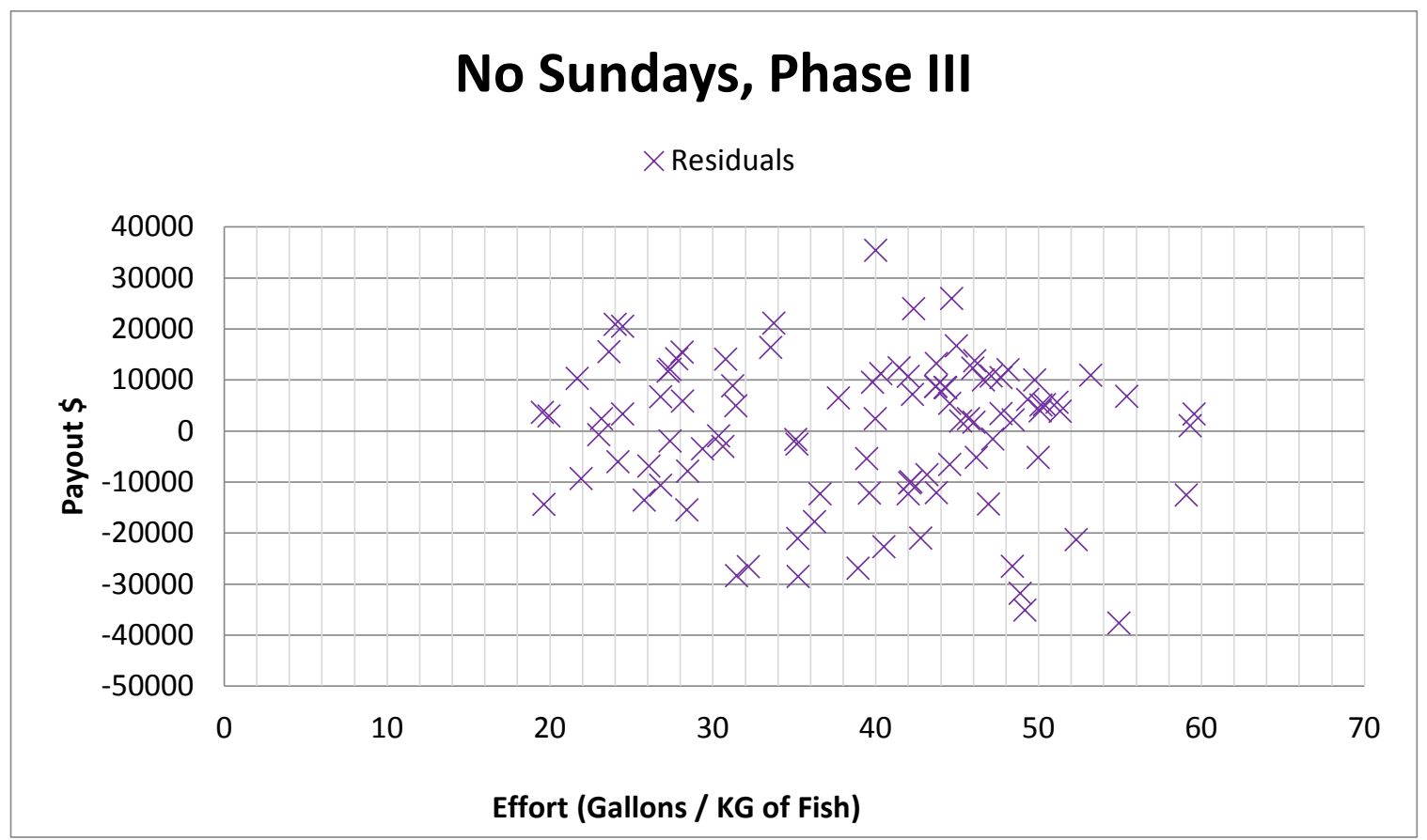

Figure 7.20: Scattering points for Residuals for No Sundays Phase III. 


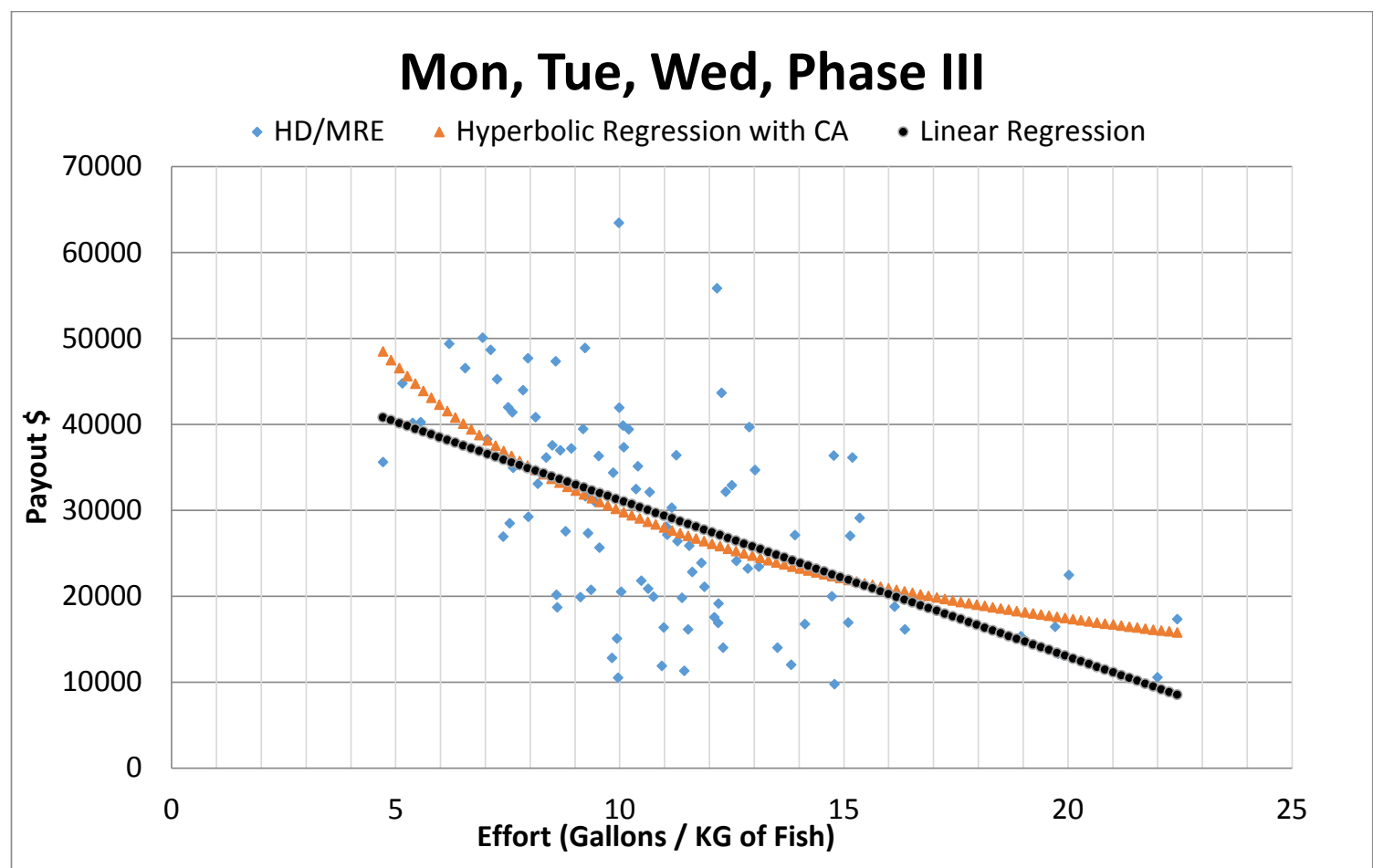

Figure 7.21: Curve fitting for Payout, Effort for MTW Phase III.

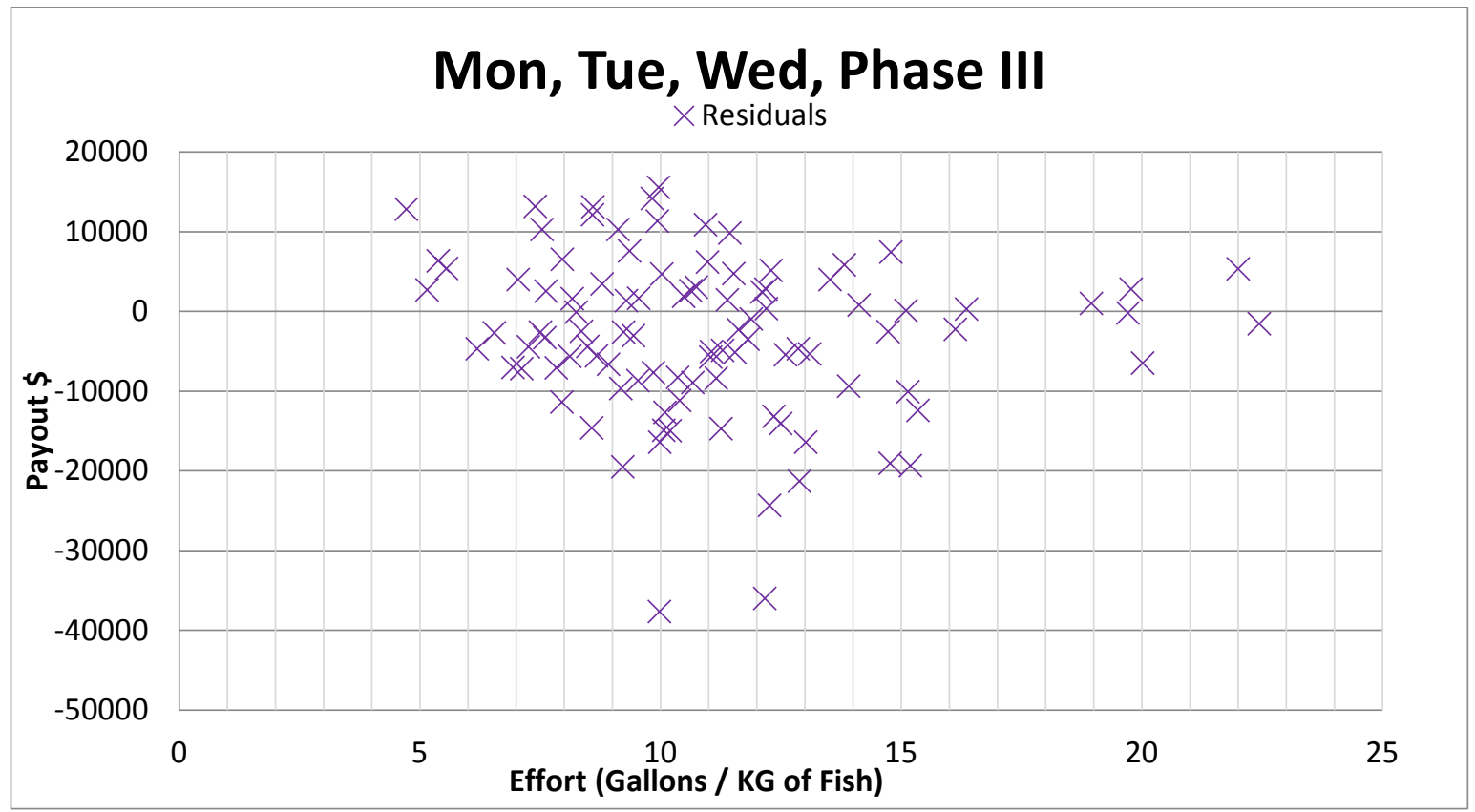

Figure 7.22: Scattering points for Residuals for MTW Phase III 


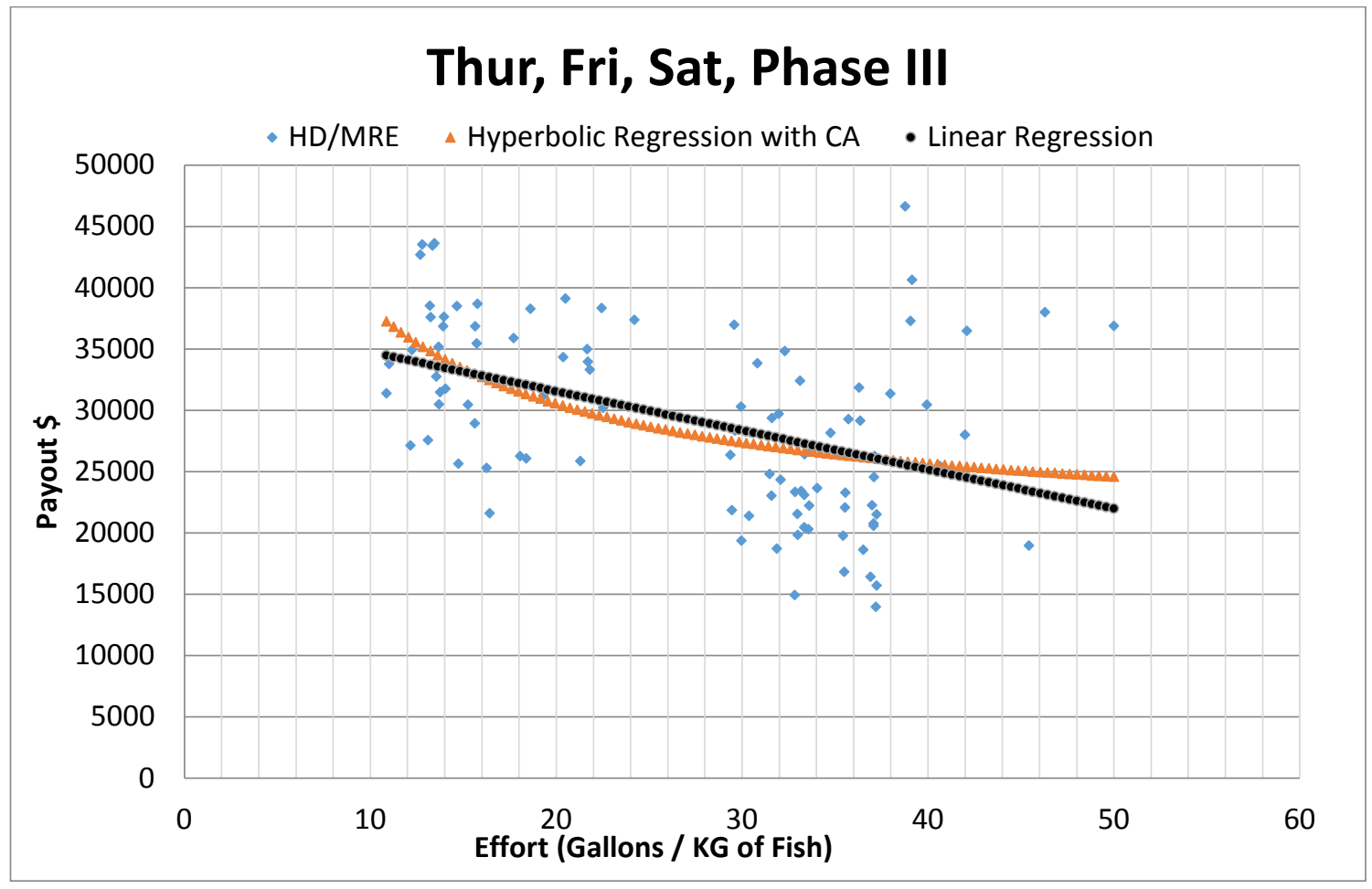

Figure 7.23: Curve fitting for Payout, Effort for RFS Phase III.

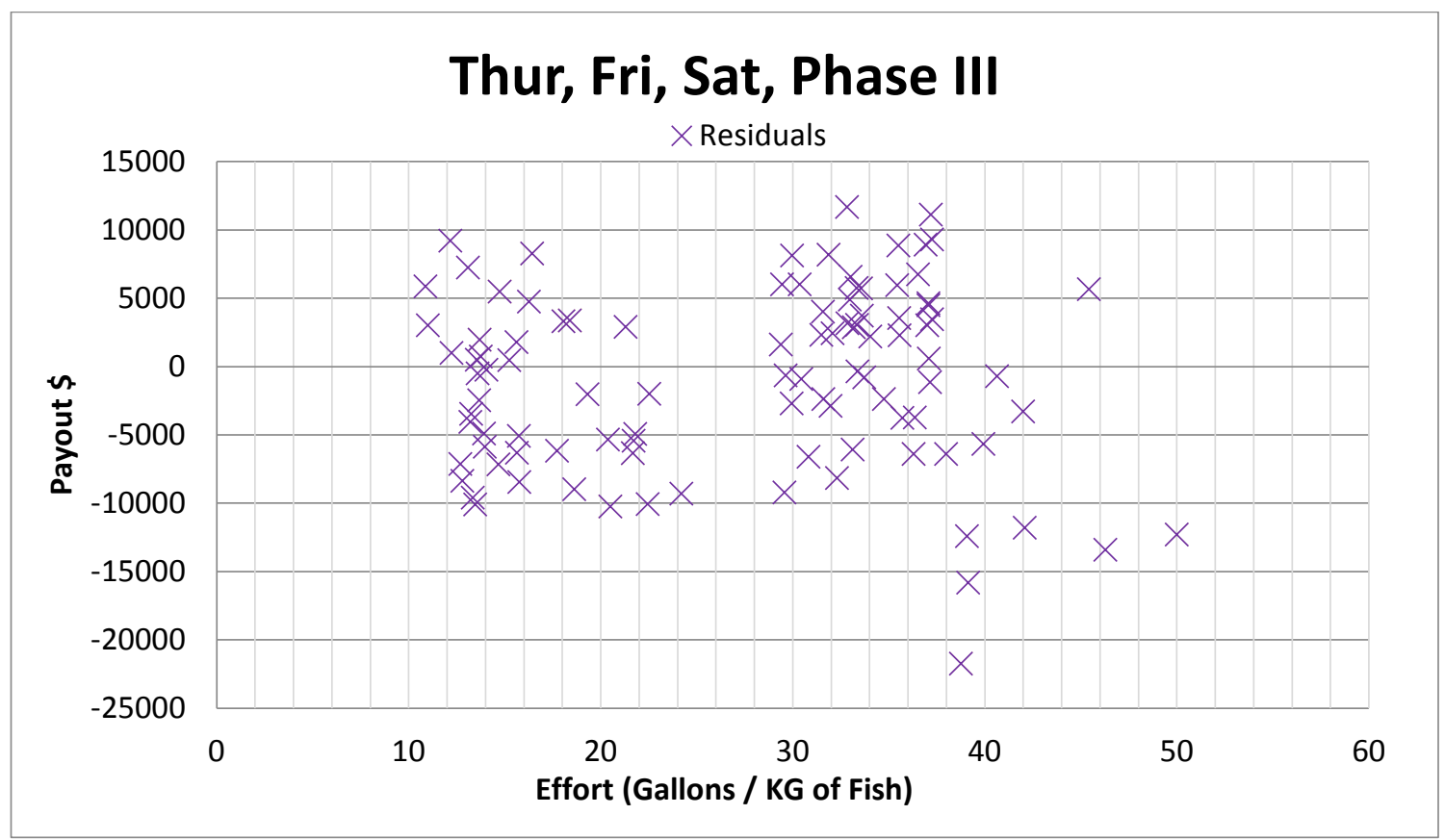

Figure 7.24: Scattering points for Residuals for RFS Phase III 


\subsection{THE SIGNIFICANT F-TEST ANALYSIS}

The statistical results of the experimental model for twelve scenarios for three phases and the four available departure days will now be presented. Table 7.1 indicates the Critical value for the F-Test at different significant levels of $\alpha$. Table 7.2 provides the summary of the results of the CAPSO runs in terms of the calculated $F$ values $F_{\text {stat }}$ for Phase I, II and Phase III.

Table 7.1: The Critical value for F-Test at different significant level of $\alpha$.

\begin{tabular}{|l|l|l|l|}
\hline $\begin{array}{c}\text { Significant } \\
\text { level } \alpha\end{array}$ & $\alpha=0.05$ & $\alpha=0.01$ & $\alpha=0.001$ \\
\hline $\mathrm{F}(\alpha$, df $1, \mathrm{df})$ & 3.07 & 4.8 & 7.5 \\
\hline
\end{tabular}

Table 7.2: Statistical value for $\left(\mathrm{F}_{\text {stat }}\right)$ for Phase I, II and Phase III.

\begin{tabular}{|c|c|c|c|c|}
\hline $\begin{array}{l}\text { Case of Fishermen } \\
\text { Trip Durations }\end{array}$ & $\begin{array}{l}\text { PHASE I } \\
\text { El Nino }\end{array}$ & $\begin{array}{l}\text { PHASE II } \\
\text { La Nina }\end{array}$ & $\begin{array}{l}\text { PHASE III } \\
\text { Back to } \\
\text { Normal }\end{array}$ & $\begin{array}{l}\mathrm{F}_{(\alpha, \mathrm{df} 1, \mathrm{df} 2)}=4.8 \\
\text { For } \alpha=0.01\end{array}$ \\
\hline Full week & 13.35 & 25.60 & 16.08 & \multirow{4}{*}{$\begin{array}{l}\text { Ho: are Rejected for } \\
\text { all phases }\end{array}$} \\
\hline No Sundays & 23.90 & 29.91 & 18.36 & \\
\hline Mon, Tue and Wed & 6.31 & 24.75 & 21.31 & \\
\hline Thur, Fri and Sat. & 7.27 & 37.25 & 14.73 & \\
\hline
\end{tabular}

Here the Null Hypothesis for a given Phase/ Day combination is that the distribution of example points is linear in form. When the Null Hypothesis H0 is true, then the variation in the sample mean for our case in the fishing scenarios (the payout as Y) provides an unbiased estimate of variance $\sigma^{2}$. However, if the Null Hypothesis H0 is false and the population means are different, then the variance in the sample means is unusually large. Now the value for calculated $F\left(F_{\text {stat }(d f 1, d f 2)}\right)$ tend to be larger than usual which means that 
we can reject $H_{0}$ for these large values of $F$, using a right-tailed statistical test as shown in the figure below. Figure 7.25 describes the area of the accepted region for $(1-\alpha)$ and the critical $\mathrm{F}$ value and the rejected region when $\left\{\mathrm{F}_{\text {stat }}>\mathrm{F}(\alpha, \mathrm{df1}, \mathrm{df2})\right\}$.

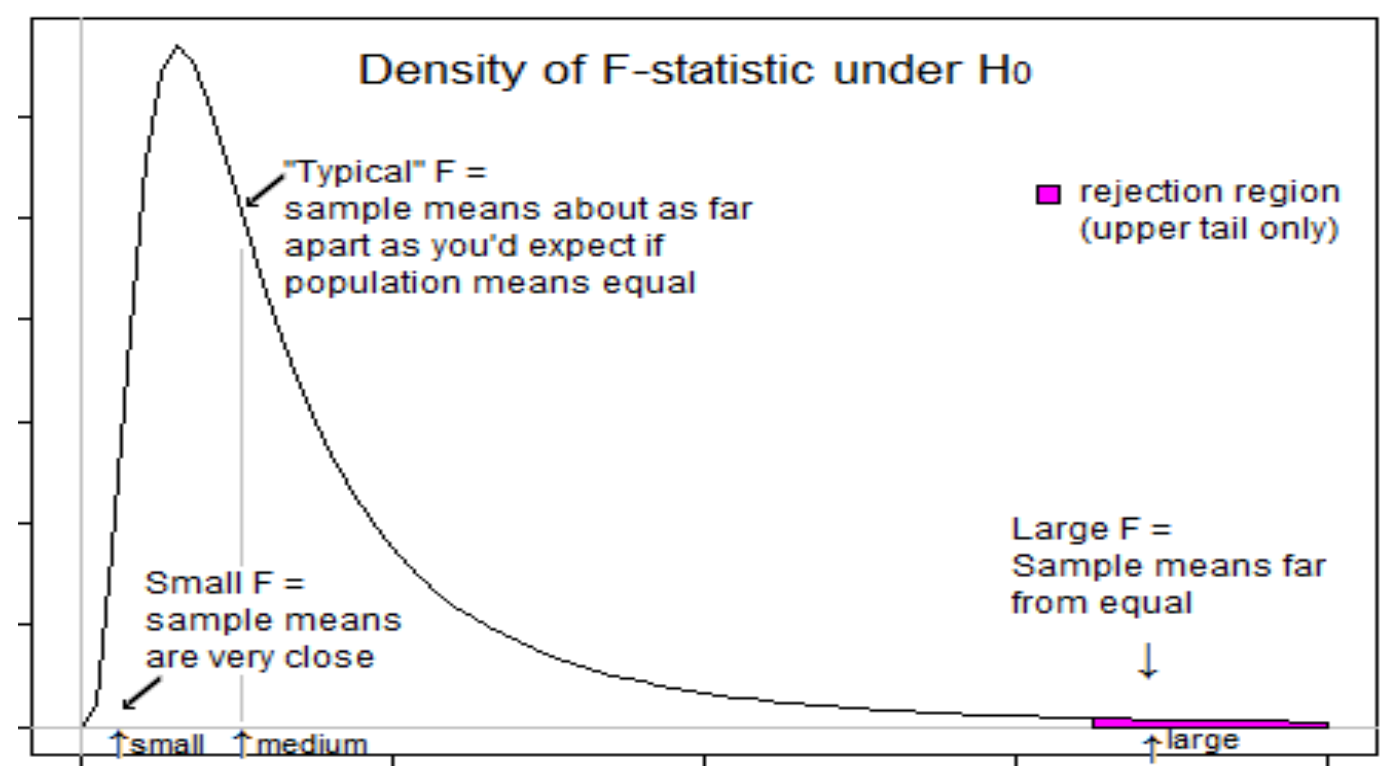

Figure 7.25: The distribution of the F statistic.

Based upon the values given in Table 7.2, the hypothesis that the distribution of all generated points is linear in nature is rejected for all Phase/Day combinations. Two Phase 1 scenarios, (Mon_Tue_Wed) and (Thur_Fri_Sat) would be rejected at $\alpha=0.001$. It is interesting to note that while in most cases for Phase I and II the non-linear model overpredicts payout with low effort whereas in El Nino the tendency is to under-predict payout for Mon_Tue_Wed and Thur_Fri_Sat which suggests a larger than anticipated crop of available catches nearby. 


\subsection{CONCLUSIONS}

In this chapter we used the Cultural Algorithm toolkit to help test whether the biobjective model exhibited a hyperlinear distribution consistent with an overall non-linear relationship between the two complementary objectives. In other words, are the Pareto fronts generated in Chapter 6 truly representative of the non-linear nature of the two goals, and if so what are the differences between those curves in terms of parabolic curves and a best fit linear function?

The results confirm the presence of a strong hyper-linear relationship between the two goals for all Phase/Day combinations. We only get close to the critical values in two instances in Phase I: Mon_Tue_Wed and Thur_Fri_Sat. The null hypothesis is easily rejected in all cases and only approaches the rejection rejoin in those two instances. In those cases, the drop-off in productivity as one moves away from Cerro Azul in terms of effort is so steep as to be approximated reasonable well by a linear model. In the other instances the hyper-linear model tends to be a good fit with the distribution of tours in all of the other cases especially for mid-range efforts. The differences that do appear are in terms of the slight over prediction for small and large effort values.

The non-linear model is the best fit in La Nina where the interaction between the variables has strong non-linearity to it based upon the changes in water temperature along with corresponding changes in catch distributions. It is also a good fit in the back to normal phase but it is clear that the agents have more predictive knowledge there. The non-linear model is the least predictive in the El Nino case, not because the distribution is overall nonlinear, but because the distribution is "box like" with a steep decline as one moves away from 
Cerro Azul and then a relative flattening out after effort reaches a critical point. This reflects the ability of agents to stay close by Cerro Azul and still collect highly desirable catches. 


\section{CHAPTER 8: CONCLUSION AND FUTURE WORK}

Recently it has been found that the earth's oceans are warming at a pace that is $40 \%$ faster than predicted by a United Nations panel a few years ago. As a result, 2019 has become the warmest year on record for the earth's oceans. That is because the oceans have acted as a buffer by absorbing $93 \%$ of the heat produced by the greenhouse gases [40].

The impact of the oceanic warming has already been felt in terms of the periodic warming of the Pacific Ocean as an effect of the ENSO process. The ENSO process is a cycle of warming and subsequent cooling of the Pacific Ocean that can last over a period of years. This cycle was first documented by Peruvian fishermen in the early 1600's. So it has been part of the environmental challenges that have been presented to economic agents throughout the world since then. It has even been suggested that the cycle has increased in frequency over the years, perhaps in response to the overall issues related to global warming.

So, although the onset of the ENSO cycle might be viewed as disruption of the fishing economy in a given area, there is some possibility that over time agents have been able to develop strategic responses to these changes to as to reduce the economic risk associated with them. In this thesis we hypothesize that at least on local level there are strategic and socially related mechanisms to mitigate the impact.

To this end we employed data collected by Marcus as part of an Archaeological site excavation project in an ancient fishing site nearby a modern Peruvian town, both named Cerro Azul. Marcus arranged with the local authorities to record the results of all fishing trips during a period of time between March 1984 and July 1986. During that time the area was in the process of emerging from one of the largest ENSOs on record. This was perceived to be a 
great opportunity to see how the collective body of fishermen was able to alter their fishing strategies to deal with these more uncertain times.

While this knowledge might be useful in the understanding and interpretation of ancient remains, our intention was mainly to use principles from Artificial Intelligence and Complex Systems to provide an understanding how what the modern responses to these changes might be. Certainly the knowledge needed to produce a collective response from a group of otherwise independent agents has emerged from generations of experience. It is the understanding of the knowledge that concerns us here.

To this end we generated a simple agent-based model of local fishing behavior that was based upon two conflicting goals:

1. One goal was to produce a Payout over time that would allow the fishermen to produce an income necessary for their survival along with hopefully some profit to them along the way.

2. The second goal was to ensure that enough resources were allocated to the trips, tours, in order to assure their success. This goal reflected the need to buffer the risk associated with the changing environment and its impact on the local economy. As stated by [35], "the goal is to just be able to keep fishing".

We then produced a database to house the data collected by Dr. Marcus in order to answer the specific questions that we had about the local fishing systems at different levels of detail. Certainly one can characterize the system, even such a spatially and temporarily situated one as a complex system about which questions could be asked about it and hypotheses tested at varying levels of detail. It was the goal of the database design to support those activities. 
The database and its structure were described in Chapter 2. In Chapter 3 we used the database to collect knowledge about the system at several levels of granularity, macro-, meso-, and micro for each of the three ENSO phases under consideration. Once we had sufficient information to realize the basic parameters of our model and its constraints we needed to have an evolutionary tool whose sole purpose was to model and simulate Cultural Change. Chapter 4 presented the tool developed to support the computational demands of this multi-objective optimization problem. It is called CAPSO and combines Cultural Algorithms and Particle Swarm models to describe the agent adaptations here [35] .

The operation agent-based model was then discussed in Chapter 5. There we reformatted the dataset as a search graph. The idea was to investigate the difference in the goals achieved by agents that are constrained to generate a sequence of tours over the entire phase that fit constraints as to when they call leave, and return. In other words, these tours reflect choices made by the fishermen in response to the current environment that they were facing. The key question is whether the simulated responses support the fact that these responses are intrinsically different in nature or just minor variations on the same theme.

In order to address this question, we generated 500 random tours and used nondominated sorting to produce approximations of the corresponding Pareto front for each of four basic tours constraint scenarios in each phase. Our basic conclusions were that the goal was to maintain a sustained level of profitability in the wake of these changes. In order to do these different search strategies were employed to buffer the impact of this increased uncertainty. This buffering effect was particularly visible in the La Nina phase. While the Payout did not drop substantially over the three phase, the shape of the Pareto curves changed due to the need to shift resources around. More resources were invested in the La 
Nina phase in order to sustain Payout due to the need to search over a wider range of sites for suitable catches. When the temperature returned to normal the related Pareto curve demonstrated that these additional resources were no longer needed as a buffer since the fishermen were now able to apply their knowledge of the local area to be more productive there.

The next question was that while theses simulations generated different tour distribution and different Pareto Front were they really all that different? In other words, could they be viewed as really just minor variations on the same search process. In order to address this question we applied the CAPSO Cultural Algorithm to generate a hyperlinear curve to describe each of the distributions and then test to see if these curves were in fact statistically different from each other. They were in fact different except for Mon_Tue_Wed and Thur_Fri_Sat in the El Nino phase. These were the lowest level scenarios modeled and the observation period for the residual El Nino was only 4 month as opposed to about a year each for the other two. So there was less opportunity to visualize the differences here even if there were.

In summary our results suggest that indeed the collective economic response of the fishermen demonstrates an ability to respond to the unpredictabilities of climate change, but at a cost. It is clear that the fishermen have gained the collective knowledge over the years to produce a coordinated response that can be observed at a higher level. Of course, this knowledge can be used to coordinate activities only if it is communicated socially within the society. Although our data does not provide any explicit information about such communication there is some indirect evidence that the adjustments in strategy are brought 
about by the increased exchange of experiences among the fishermen. We see that indirectly in two basic ways:

1. The distributions seem to suggest dominant and successor waves of strategies that may be associated with the length of time over which the simulation window is conducted. These waves were suggested to represent how subsequent trips were influenced by knowledge brought back by agents from trips the days right before that trip. For example, Dr. Marcus recalls one brother talking to another brother about his fishing experiences. This knowledge might be shared with close blood relatives or might be conveyed in general to others. It is hard for us to say at this point.

2. Our discussion of the meso and micro-statistics in Chapter 3 suggested that indeed there were at least two waves present in terms of the amount of catches returned in certain phases relative to certain types of catches. For example, there was often one that started on Monday and peaked on Wednesday, while a second started on Thursday and peaked on Friday or Saturday.

In future work, we plan in use the Social Network capabilities of Cultural Algorithms to attempt to model the impact that knowledge acquisition and its subsequent distribution has on strategic decision-making. 


\section{REFERENCES}

[1] J. Marcus, Coastal Ecosystems and Economic Strategies at Cerro Azul, Peru: The Study of a Late Intermediate Kingdom. Memoir 59, Ann Arbor, Michigan: Museum of Anthropolgy, University of Michigan, 2016.

[2] R. G. Reynolds, An Adaptive Computer Model of the Evolution of Agriculture, Ann Arbor: University of Michigan, 1979.

[3] K. Flannery, "The Cultural Evolution of Civilizations," 1972 Annual Review of Ecology and Systematics, pp. 399-426, 1972.

[4] R. G. Reynolds, "On Modeling the Evolution of Hunter-Gatherer Decision-Making Systems," Geographical Analysis, vol. 10, pp. 31-46, 1978.

[5] R. G. Reynolds and A. Lazar, "Simulation the Evolution of Archaic States," in Proceedings of the 2002 Congress on Evolutionary Computation, Honolulu, 2002.

[6] Z. Kobti, Learning in Dynamic Hierarchical Network Structures in Complex Systems a Study on the effect of Learning in Improving Resilience in Large Level Social Networks, Detroit: Wayne State University, 2004.

[7] Wikipedia, "Wiki/Cerro_Azul,_Peru," 1210 2018. [Online]. Available: https://en.wikipedia.org/wiki/Cerro_Azul,_Peru. [Accessed 1210 2018].

[8] J. H. Holland, Adaptation in Natural and Artificial Systems, Ann Arbor: University of Michigan Press, 1975.

[9] R. Patrick, R. Robertson, T. Zhu and S. Steinschneider, "The Effects of Variations in El Nino and La Nina Patterns on World Food Markets," in American Geographic 
Union Fall Meeting Abstracts, 2014.

[10] NOAA, "ENSO," National Oceanic and Atmospheric Administration, 1 December 2018. [Online]. Available: https://www.climate.gov/enso.

[11] J. Marcus, Late Intermediate Occupation at Cerro Azul, Peru, Ann Arbor: University of Michigan Museum of Anthropology, 1987.

[12] J. Tarazona, "Positive Effects of "El Niño" on Macrobenthos Inhabiting Hypoxic Areas of the Peruvian Upwelling System," Oecoloiga, pp. 184-190, 1988.

[13] J. Marcus, Excavations at Cerro Azul, Peru: The Architecture and Pottery, Los Angeles: UCLA Cotsen Institute of Archaeology, 2008.

[14] A. Engelbrecht, Computational Intelligence: An Introduction, New York: John Wiley and Sons, 2007.

[15] R. G. Reynolds, "An Introduction to Cultural Algorithms," in Proceedings of the Third Annual Conference on Evolutionary Programming, 1994.

[16] D. Liu, Multi-Objective Cultural Algorithms, Detroit: Wayne State University, 2011.

[17] L. Wirth, A Bibliography of the Urban Community, Chicago: University of Chicago Press, 1925, pp. 161-228.

[18] R. E. Blanton, Monte Albán: Settlement Patterns at the Ancient Zapotec Capital, Academic Press, 1978.

[19] T. Jayyousi, Bringing to Life an Ancient Urban Center at Monte Alban, Mexico: Exploiting the Synergy Between the Micro, Meso, and Macro Levels in a Complex System, Detroit: Wayne State University, 2012. 
[20] K. Kattan, R. G. Reynolds and J. Marcus, "Artisanal Fishing at Cerro Azul," in Coastal Ecosystems and Economic Strategies at Cerro Azul, Peru: The Study of a Late Intermediate Kingdom, Museum of Anthropology, University of Michigan, 2016, pp. 352-358.

[21] E. Miraglia and R. Law, "A Baseline Definition of Culture," Washington State University, Virtual Campus, The Learning Commons, Washington D.C., 1996.

[22] M. Ali, Using Cultural Algorithms to Solve Optimization Problems with a Social Fabric Approach, Detroit: Wayne State University, 2008.

[23] Y. Shi, "Brain Storm Optimization Algorithm," in International Conference in Swarm Intelligence, Berlin, 2011.

[24] X. Che, Weaving the Social Fabric: Optimization Problem Solving in Cultural Algorithms using the Cultural Engine, Detroit: Wayne State University, 2009.

[25] C. Chung and R. G. Reynolds, "A Test bed for Solving Optimization Problems using Cultural Algorithms," in Proceedings of the Fifth Annual Conference on Evolutionary Programming, Boston, 1996.

[26] N. Clayton, D. Griffiths and A. Dickinson, "Declarative and Episodic-like Memory in Animals: Personal Musings of a Scrub Jay," in The Evolution of Cognition, Boston. MA, The MIT Press, 2001.

[27] X. Jin and R. G. Reynolds, "Using Knowledge-based Evolutionary Computation to Solve Nonlinear-constraint Optimization Problems: a Cultural Algorithm Approach," in Proceedings of the 1999 Congress on Evolutionary Computation, Washington D.C., 
1999.

[28] S. Saleem and R. G. Reynolds, "Cultural Algorithms in Dynamic Environments," in Proceedings of the IEEE Congress on Evolutionary Computation, 2000.

[29] R. G. Reynolds and S. M. Saleem, "The Impact of Environmental Dynamics on Cultural Emergence," in Perspectives on Adaptation in Natural and Artificial Systems, Oxford University Press, 2005, pp. 253-280.

[30] S. Saleem, Knowledge-Based Solution to Dynamic Optimization Problems Using Cultural Algorithms, Detroit: Wayne State University, 2001.

[31] B. Peng, Knowledge Swarms in Cultural Algorithms for Dynamic Environments, Detroit: Wayne State University, 2005.

[32] K. Flannery, The Early Mesoamerican Village, Left Coast Press, 2009.

[33] R. Iacoban, The Synergy of the Knowledge Factors of Cultural Algorithm When Applied to Static and Dynamic Environments, Detroit: Wayne State University, 2002.

[34] C. Best, Multi-Objective Cultural Algorithms, Detroit: Wayne State University, 2009.

[35] S. D. Stanley, K. Kattan and R. G. Reynolds, "CAPSO: A Parallelized Multi-Objective Cultural Algorithm Particle Swarm Optimizer," in 2019 Proceedings of IEEE Congress on Evolutionary Computation, New Zealand, 2019.

[36] J. Kennedy and R. Eberhart, "Particle Swarm Intelligence," in 1995 Proceedings of IEEE International Conference on Neural Networks, Piscataway, 1995.

[37] J. D. Schaffer, "Multiple Objective Optimization with Vector Evaluated Genetic Algorithms," in Proceedings of the First International Conference on Genetic 
Algorithms and Their Applications, 1985.

[38] Wikipedia, "Wiki/Artisanal Fishing," 2018. [Online]. Available: https://en.wikipedia.org/wiki/Artisanal_fishing.

[39] Wikipedia, "Wiki/Pareto Front," 1110 2018. [Online]. Available: https://en.wikipedia.org/wiki/Pareto Front.

[40] R. G. Reynolds and H. Al-Shehri, "The Use of Cultural Algorithms with Evolutionary Programming to Guide Decision Tree Induction in Large Databases," in 1998 Proceedings of the IEEE International Conference on Evolutionary Computation Proceedings, Anchorage, 1998.

[41] L. J. Cheng, Z. Abraham and K. Trenberth, "How Fast are the Oceans Warming?," Science, pp. 128-129, 2019.

[42] D. B. Fogel, "Evolving Behaviors in the Iterated Prisoner's Dilemma," Evolutionary Computation, pp. 77-97, 1993.

[43] I. Millington, Artificial Intelligence for Games, Morgan Kaufmann, 2006.

[44] J. M. Smith, Evolution and the Theory of Games, Cambridge University Press, 1982.

[45] A. Rapoport, Two-Person Game Theory, Courier Dover Publications, 1999.

[46] I. Borg and P. Groenen, Modern Multidimensional Scaling, Theory and Applications, New York: Springer-Verlag, 1997.

[47] S. Ashish and M. Srivastava, Regression Analysis: Theory, Methods, and Applications, New York: Springer, 1997.

[48] Wikipedia, "Wiki/Learning_curve," 1110 2018. [Online]. Available: 
https://en.wikipedia.org/wiki/Learning_curve.

[49] A. Lazar, Impact of Heuristic Knowledge Discovery Techniques On Multi-Agent Simulation of Cultural Evolution, Detroit: Wayne State University, 1992.

[50] H. Al-Shehri, Evolution-Based Decision-Tree Optimization Using Cultural Algorithms, Detroit: Wayne State University, 1997.

[51] J. Barceló, Computational Intelligence in Archaeology, IGI Global, 2008.

[52] K. Deb and H. Jain, "An Evolutionary Many-Objective Optimization Algorithm Using Reference-Point-Based Nondominated Sorting Approach, Part I: Solving Problems With Box Constraints," in IEEE Transactions on Evolutionary Computation, 2014.

[53] T. Jayyousi, Using Evolutionary Computation and Data-mining to Model the Emergence of Archaic Urban Centers, Detroit: Wayne State University, 2008.

[54] C. Chung and R. G. Reynolds, "CAEP: An Evolution-based Tool for Real-valued Function Optimization Using Cultural Algorithms," International Journal on Artificial Intelligence Tools, vol. 7(3), pp. 239-291, 1998.

[55] R. G. Reynolds and A. Nazzal, "Using Cultural Algorithms with Evolutionary Computing to Extract Site location Decisions from Spatio-Temporal Databases," in International Conference on Evolutionary Programming, 1997.

[56] R. Axelrod, The Evolution of Cooperation, New York: Basic Books, 1984.

[57] D. E. Goldberg, Genetic Algorithms in Search, Optimization and Machine Learning, Boston: Addison Wesley, 1989.

[58] D. Mark, Behavioral Mathematics for Game AI, Charles River Media, 2009. 
[59] S. Deam da Silva, O. Teixeria and R. de Oliveria, Performance Study of Cultural Algorithms Based on Genetic Algorithm with Single and Multi-Population for the MKP, IntechOpen, 2012.

[60] C. Cioffi-Revilla, K. D. Jong and J. K. Basset, "Evolutionary Computation and AgentBased Modeling: Biologically-inspired Approaches for Understanding Complex Social Systems," Computational and Mathematical Organization Theory, pp. 356-373, 2012.

[61] K. Deb, L. Thiele, M. Laumanns and E. Zitzler, "Scalable Multi-Objective Optimization Test Problems," in Proceedings of the 2002 Congress on Evolutionary Computation, Honolulu, 2002.

[62] C. Renzhi, K. Li and X. Yao, "Dynamic Multi-Objectives Optimization with a Changing Number of Objectives," IEEE Transactions on Evolutionary Computation, vol. 22, pp. 157-171, 2018.

[63] R. G. Reynolds, Culture on the Edge of Chaos: Cultural Algorithms and the Foundations of Social Intelligence, Springer, 2018.

[64] K. Flannery, J. Marcus and R. Reynolds, The Flocks of the Wamani, New York: Routledge, 2009.

[65] CNET, "everything-you-need-to-know-about-the-godzilla-el-nino/," 31 July 2015. [Online]. Available: https://www.cnet.com/pictures/everything-you-need-to-knowabout-the-godzilla-el-nino/.

[66] S. Phelps, M. Peter and S. Parsons, "Evolutionary Mechanism Design: A Review," Autonomous Agents and Multi-agent Systems, vol. 21, no. 2, pp. 237-264, 2010. 
[67] P. Franzel, The Data-mining and Visualization of Decision Rules for Site Selection and Utilization in an Archaic Urban Center: A Monte Albán Example, Detroit: Wayne State University, 2007.

[68] J. Koza, "Hierarchical Genetic Algorithms Operating on Populations of Computer Programs," in Proceedings of the Eleventh International Joint Conference on Artificial Intelligence, 1989.

[69] C. G. Langton, Life At the Edge of Chaos, Addison-Wesley, 1992, pp. 41-92.

[70] T. Baarslag, K. Hindriks, C. K. S. Jonker and R. Lin, "The First Automated Negotiating Agents Competition (ANAC 2010)," in New Trends in Agent-Based Complex Automated Negotiations, Heidelberg, 2012.

[71] M. Broom and R. J, Game-Theortical Models in Biology, CRC Press, 2013.

[72] M. Dorigo, "The Ant System: Optimization By a Colony of Cooperating Agents," in 1996 Proceedings of IEEE Transactions on System, Man, and Cybernetics, 1996.

[73] J. Marcus, "On the Nature of the Mesoamerican City," in Essays in Honor of Gordon R. Willey, Albuquerque, 1983, pp. 195-242.

[74] R. J. Dzeng and Y. C. Lin, "Intelligent Agents for Supporting Construction Procurement Negotiation," Expert System with Applications, vol. 27, pp. 107-119, 2004.

[75] B. Peng, R. G. Reynolds and J. Brewster, "Cultural Swarms," in 2003 Proceedings of IEEE 2003 Congress on Evolutionary Computation, Canberra, 2003.

[76] C. C. A. Coello, G. B. Lamont and D. A. Van Veldhuizen, Evolutionary Algorithms for 
Solving Multi-Objective Problems, New York: Springer, 2007.

[77] E. Zitzier and L. Thiele, "Multi-Objective Evolutionary Algorithms: A Comparative Case Study and the Strength Pareto Approach," IEEE Transctions on Evolutionary Computation, pp. 257-271, 1999.

[78] W. Hamilton, "The Genetic Evolution of Social Behavior I and II," Journal of Theoretical Biology, pp. 17-52, 1964.

[79] P. Hammerstein and R. Selten, Game Theory and Evolutionary Biology, Elsevier Science, 1994.

[80] P. Hingston and G. Kendall, "Learning Versus Evolution in Iterated Prisoner's Dilemma," in Proceedings of the 2004 Congress on Evolutionary Computation, Portland, 2004.

[81] J. H. Holland, ECHO: Explorations of Evolution in a Miniature World, Redwood City: Addison-Wesley, 1990.

[82] T. Jayyousi and R. G. Reynolds, "Using Cultural Algorithms to Generate Models for Archaic Urban Centers: The Monte Alban example," in 2013 IEEE Congress on Evolutionary Computation, Cancun, 2013.

[83] A. Kattan, Y. S. Ong and E. Galvan-Lopez, "Multi-Agent Multi-Objectives Negotiations with Incomplete Information: A Genetic Algorithm Based on Discrete Surrogate Approach," in 2013 IEEE Congress on Evolutionary Computation, Cancún, 2013.

[84] A. Konak, D. Coit and A. Smith, "Multi-Objective Optimization Using Genetic 
Algorithms: A Tutorial," Elsevier, Reliability Engineering and System Safety, no. 91, pp. 992-1007, 2006.

[85] J. F. Nash, "Equilibrium Points in N-Person Games," in Proceedings of National Academy of Sciences 36, 1950.

[86] Macal and M. J. North, "Tutorial on Agent-Based Modeling and Simulation," Journal of Simulation, pp. 151-162, 2010.

[87] M. J. Osborne and A. Rubinstein, A Course in Game Theory, MIT Press, 1994.

[88] D. Ostrowski and R. G. Reynolds, "Knowledge-Based Software Testing Agent Using Evolutionary Learning with Cultural Algorithms," in Proceedings of the 1999 Congress on Evolutionary Computation, Washington D.C., 1999.

[89] R. J. Quinlan, "Induction of Decision Trees," Machine Learning, vol. 1, no. 1, pp. 81$106,1986$.

[90] R. G. Reynolds and M. Ali, "Computing With the Social Fabric: The Evolution of Social Intelligence within a Cultural Framework," IEEE Computational Intelligence Magazine, pp. 18-30, 2008.

[91] R. G. Reynolds and B. Peng, "Cultural Algorithms: Modeling of How Cultures Learn to Solve Problems," in 16th IEEE International Conference on Tools with Artificial Intelligence, Boca Raton, 2004.

[92] R. G. Reynolds and R. S. Alomari, "Cultural Evolution of Ensemble Learning for Problem Solving," in 2006 Congress on Evolutionary Computation, 2006.

[93] R. G. Reynolds and C. Chung, "Fuzzy Approaches to Acquiring Experimental 
Knowledge in Cultural Algorithms," in Proceedings of the Ninth IEEE International Conference on Tools with Artificial Intelligence, Newport Beach, 1997.

[94] R. G. Reynolds and S. Zhu, "Knowledge-Based Function Optimization using Fuzzy Cultural Algorithms with Evolutionary Programming," in 2001 Proceedings of IEEE Transactions on Systems, Man, and Cybernetics, 2001.

[95] R. G. Reynolds, M. Ali and J. Thaer, "Mining the Social Fabric of Archaic Urban Centers with Cultural Algorithms," Computer, vol. 41, no. 1, pp. 64-72, 2008.

[96] R. G. Reynolds, M. Ali and R. Alomari, "Optimization Problem Solving Using Predator/Prey Games and Cultural Algorithms," in 2006 IEEE Symposium on Computational Intelligence and Games, Reno, 2006.

[97] R. G. Reynolds and L. Kinnaird-Heether, "Optimization Problem Solving with Auctions in Cultural Algorithms," Memetic Computing 5, pp. 83-94, 2013.

[98] R. G. Reynolds and W. Sverdlik, "Problem Solving using Cultural Algorithms," in 1994 Proceedings of the First IEEE Conference on Evolutionary Computation, Florida, 1994.

[99] L. J. Fogel, A. J. Owens and M. J. Walsh, Artificial Intelligence Through Simulated Evolution, Wiley, 1996.

[100] R. H. Guttman and P. Maes, "Cooperative vs. Competitive Multi-Agent Negotiations in Retail Electronic Commerce," in Proceedings of the Second International Workshop on Cooperative Information Agents, Paris, 1998.

[101] A. Lazar and R. G. Reynolds, "Heuristic Knowledge Discovery for Archaeological 
Data Using Cultural Algorithms and Rough Sets," in Heuristics and Optimization for Knowledge Discovery, Kluwer Academic Press, 2003.

[102] M. J. North and T. R. Howe, "The Repast Symphony Development Environment," in 2005 Conference on Generative Social Processes, Models, and Mechanisms, 2005.

[103] R. G. Reynolds, "Version Space Controlled Genetic Algorithms," in 1991 Proceedings of the Second Annual Conference on AI, Simulation and Planning in High Autonomy Systems, 1991.

[104] J. Von Neumann and O. Morgenstern, Theory of Games and Economic Behavior, John Wiley and Sons, 1944. 


\section{ABSTRACT \\ THE USE OF CULTURAL ALGORITHMS TO LEARN THE IMPACT OF CLIMATE ON LOCAL FISHING BEHAVIOR IN CERRO AZUL PERU \\ by}

\section{KHALID KATTAN}

May 2019

Advisor: Dr. Robert G. Reynolds

Major: Computer Science

Degree: Doctor of Philosophy

Recently it has been found that the earth's oceans are warming at a pace that is $40 \%$ faster than predicted by a United Nations panel a few years ago. As a result, 2019 has become the warmest year on record for the earth's oceans. That is because the oceans have acted as a buffer by absorbing $93 \%$ of the heat produced by the greenhouse gases [40].

The impact of the oceanic warming has already been felt in terms of the periodic warming of the Pacific Ocean as an effect of the ENSO process. The ENSO process is a cycle of warming and subsequent cooling of the Pacific Ocean that can last over a period of years. This cycle was first documented by Peruvian fishermen in the early 1600's. So it has been part of the environmental challenges that have been presented to economic agents throughout the world since then. It has even been suggested that the cycle has increased in frequency over the years, perhaps in response to the overall issues related to global warming.

Although the onset of the ENSO cycle might be viewed as disruption of the fishing economy in a given area, there is some possibility that over time agents have been able to develop strategic responses to these changes to as to reduce the economic risk associated with them. During that time the Cerro Azul, Peru was in the process of emerging from one of 
the largest ENSOs on record. This was perceived to be a great opportunity to see how the collective bodies of fishermen were able to alter their fishing strategies to deal with these more uncertain times.

Our results suggest that indeed the collective economic response of the fishermen demonstrates an ability to respond to the unpredictabilities of climate change, but at a cost. It is clear that the fishermen have gained the collective knowledge over the years to produce a coordinated response that can be observed at a higher level. Of course, this knowledge can be used to coordinate activities only if it is communicated socially within the society. Although our data does not provide any explicit information about such communication there is some indirect evidence that the adjustments in strategy are brought about by the increased exchange of experiences among the fishermen. 


\section{AUTOBIOGRAPHICAL STATEMENT}

Khalid Kattan joined the Department of Computer Science at Wayne State University in 2006 to purse his PhD. In 2008, he joined the Artificial Intelligence team, directed by Dr. Robert G. Reynolds. During his studies, Khalid worked full time in the information technology sector outside of academia, most recently as a SAP Authorization and Security Specialist, at Autoneum Corporation, a Tier I Automotive Supplier. Khalid found his passion for teaching when he was a full time visiting lecturer at the Computer Science Department of the University of Massachusetts, Dartmouth, from 2001 to 2005. He received his MS in Computer Science with his thesis title "Minimizing Job Control Time Using Petri Nets",

from the University of Massachusetts, Dartmouth USA, 2001. He received his bachelor's in Business Administration with a minor in Management Information Systems from Tennessee Technological University in 1996.

Khalid enjoys research areas in artificial intelligence, especially those dealing with predictive behavior. 